Florida International University FIU Digital Commons

$10-18-2012$

\title{
Knowledge, Attitudes, and Practices Affecting Health Behaviors in the U.S. Army Special Operations Command Population of Rangers
}

\author{
Michael J. Pagel \\ Florida International University, mpage006@fiu.edu
}

DOI: $10.25148 /$ etd.FI12110701

Follow this and additional works at: https://digitalcommons.fiu.edu/etd

\section{Recommended Citation}

Pagel, Michael J., "Knowledge, Attitudes, and Practices Affecting Health Behaviors in the U.S. Army Special Operations Command Population of Rangers" (2012). FIU Electronic Theses and Dissertations. 717.

https://digitalcommons.fiu.edu/etd/717 


\title{
FLORIDA INTERNATIONAL UNIVERSITY \\ Miami, Florida
}

\section{KNOWLEDGE, ATTITUDES, AND PRACTICES AFFECTING HEALTH BEHAVIORS IN THE U.S. ARMY SPECIAL OPERATIONS COMMAND POPULATION OF RANGERS}

\author{
A dissertation submitted in partial fulfillment of the \\ requirements for the degree of \\ DOCTOR OF PHILOSOPHY \\ in \\ PUBLIC HEALTH \\ by \\ Michael J. Pagel \\ 2012
}


To: Interim Dean Michele Ciccazzo

R. Stempel College of Public Health and Social Work

This dissertation, written by Michael J. Pagel, and entitled Knowledge, Attitudes, and Practices Affecting Health Behaviors in the U.S. Army Special Operations Command Population of Rangers, having been approved in respect to style and intellectual content, is referred to you for judgment.

We have read this dissertation and recommend it be approved.

Richard Palmer

Gilbert Ramirez

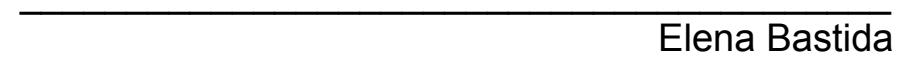

H. Virginia McCoy, Major Professor

Date of Defense: October 18, 2012

The dissertation of Michael J. Pagel is approved.

Interim Dean Michele Ciccazzo

R. Stempel College of Public Health and Social Work

Dean Lakshmi N. Reddi

University Graduate School

Florida International University, 2012 


\section{DEDICATION}

I dedicate this dissertation to the brave men of the 75th Ranger Regiment. These men

put their lives in harm's way each and every day to preserve the freedoms and way of life that each of us enjoy. 


\section{ACKNOWLEDGMENTS}

I wish to thank the members of the United States Army Special Operations Command for supporting this endeavor to conduct research within the 75th Ranger Regiment. In particular, I wish to thank Lieutenant General Charles Cleveland for recommending my participation into the Long Term Health Education program, which began my journey to attain a PhD. His support and faith in me has been the most driving force in my success thus far.

I would also like to thank Colonel Rocky Farr, Colonel Russ Kotwal, and Major Scott Gilpatrick for their unwavering support from the very beginning of my research proposal. It was up to them to accept my proposal as beneficial to the Special Operations Command, the United States Army Special Operations Command, and the 75th Ranger Regiment. Researching this population of Rangers would not have been possible without their support.

Finally I wish to thank the members of Walter Reed Army Institute of Research, the US Military HIV Research Program (MHRP), and the US Army Public Health Command. Those in particular that deserve my special thanks include: Dr. Paul Scott, Dr. Nicole Close, Colonel Steven Cersovsky, and Lieutenant Colonel (Retired) Eric Milstrey. These individuals gave this research the full benefit of their vast professional military research experience and credentials. They were all truly inspirational during the conceptualization, operationalization, and analysis phases of this research.

The members of the MHRP Data Coordinating and Analysis Center, of which Mr. Greg Pfeiffer was my most influential collaborator, were also critical during the development and construction of both the survey instrument and the data base. This is a fantastic group of professionals.

Funding for this study was from the Military Infectious Disease Research Program, executed via the U.S. Military HIV Research Program Department of Epidemiology and Threat 
Assessment. This funding would not have been possible without the complete support and tireless efforts of Dr. Paul Scott; to whom I am forever grateful.

I would also like to thank the members of my committee: Dr. H. Virginia McCoy, Dr. Elena Bastida, Dr. Gilbert Ramirez, and Dr. Richard Palmer for their support, guidance, and patience. In particular, my major professor Dr. H. Virginia McCoy deserves special thanks. She has been assisting and guiding me since prior to my being admitted into the graduate school, when I was actually serving at a combat medical clinic in Iraq. She has truly been supporting me from the beginning of all this and for that I will also be forever grateful.

Above all, I thank God I am blessed with my wife Maggie, my daughter Warner, and my son Matthew. They all stood strong with me during every good and bad moment, and have endured everything I have in so many ways. I love them all. 


\title{
ABSTRACT OF THE DISSERTATION
}

KNOWLEDGE, ATTITUDES, AND PRACTICES AFFECTING HEALTH BEHAVIORS IN THE U.S. ARMY SPECIAL OPERATIONS COMMAND POPULATION OF RANGERS

by

\author{
Michael J. Pagel
}

Florida International University, 2012

Miami, Florida

Professor H. Virginia McCoy, Major Professor

Malaria is a threat to United States military personnel operating in endemic areas, from which there have been hundreds of cases reported over the past decade. Each of these cases might have been avoided with proper adherence to malaria chemoprophylaxis medications. Military operations may detract from the strict $100 \%$ adherence required of these preventive medications. However, the reasons for non-adherence in military populations are not well understood. This behavior was investigated using a cross sectional study design on a convenience sample of U.S. Army Ranger volunteers $(n=150)$ located at three military instillations. Theoretical support was based on components of the Health Belief Model, the Theory of Reasoned Action/Theory of Planned Behavior, and the Social Cognitive Theory.

Data on knowledge, attitudes, and practices, as well as multiple environmental domains was collected using an original yet unvalidated questionnaire. The data was analyzed using bivariate Pearson correlations, binary logistic regression, and moderated logistic regressions employing a 0.05 criterion of statistical significance. Power analyses predicted $96-98 \%$ power for this analysis.

Multiple significant medium strength Pearson correlation coefficients were identified relative to the two dependent variables Take medications as directed and Intend to take the medications as directed the next time. Binary logistic regression analyses identified multiple 
variables that may predict behavioral intentions to adhere to these preventive medications, as a proxy for behavioral change. Moderated logistic regression analyses identified Command Support for adherence to these medications as a potential significant moderator that interacts with independent variables within three domains of the survey questionnaire.

The findings indicate that there may be potential significant beneficial effects, which may improve this behavior in this population of Rangers through 1) promoting affirmative interpersonal communications that emphasize adherence to these medications, 2) including malaria chemoprophylaxis medications in the mission planning process, and 3) military command support, in the form of including the importance of proper adherence to these medications in the unit safety briefings. 


\section{TABLE OF CONTENTS}

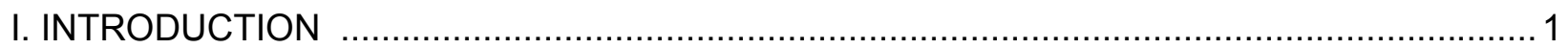

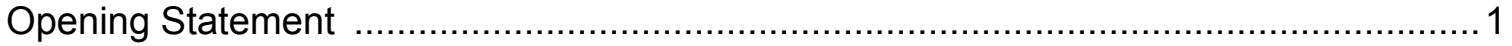

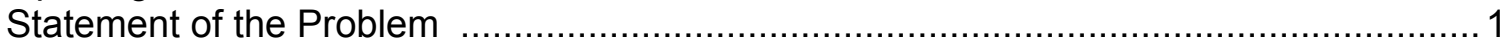

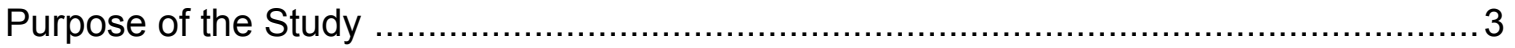

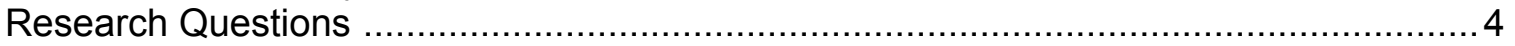

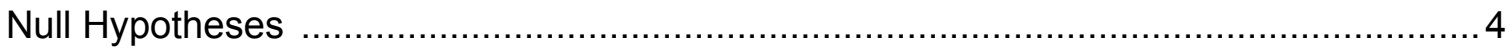

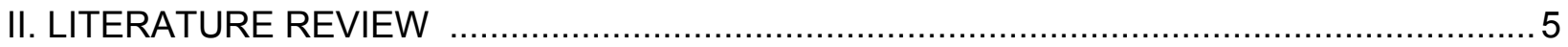

The Roadmap: A Guide through the Literature Review ………............................. 5

Background on Malaria and Malaria Epidemiologic Data ……................................... 6

Non-Medical Malaria Preventive Measures ……………..................................... 8

Malaria Chemoprophylaxis Medications, General Medical Considerations ..................... 8

Specific Malaria Chemoprophylaxis Medications ……………................................. 9

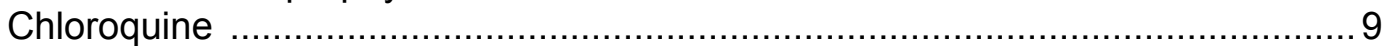

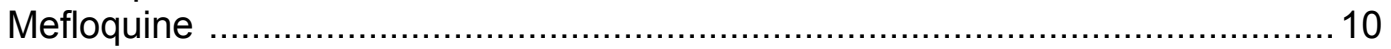

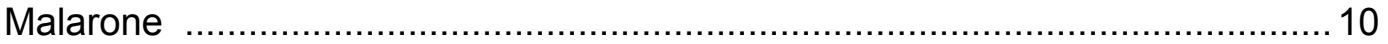

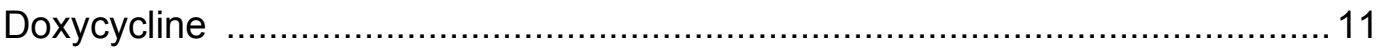

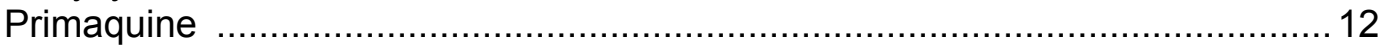

Defining Adherence and Compliance ............................................................... 12

The Most Appropriate Term in Military Populations: Adherence vs. Compliance .......... 13

Is Compliance Ever the Proper Term? ............................................................. 14

Quantifying Adherence .............................................................................. 14

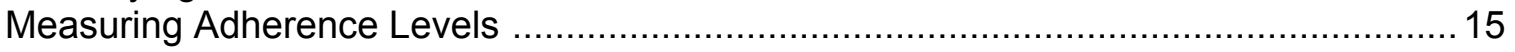

Civilian Adherence to Preventive Malaria Chemoprophylaxis Medications ……........... 15

Military Adherence to Preventive Malaria Chemoprophylaxis Medications …................ 16

A Case Study with No Cases: A Directly Observed Therapy Perspective Within

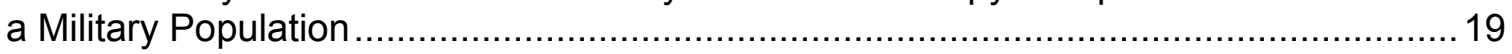

Other Directly Observed Therapy Findings ……………..................................... 20

Phenomena Affecting Adherence to General Medical Therapies ................................22

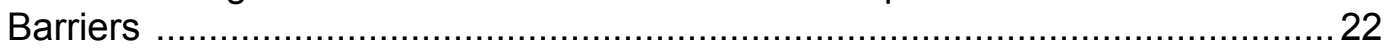

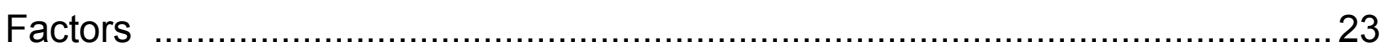

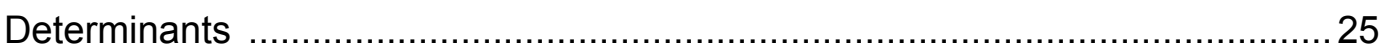

What are "not" considered determinants? …………..................................26

Costs Associated With Non-adherence and Poor Adherence ….............................2

Theoretical Models Used to Support Adherence to General Medical Therapies …........28

Strategies of Improving Adherence to General Medical Therapies …….......................2. 29

Phenomena Affecting Adherence to Malaria Chemoprophylaxis Medications

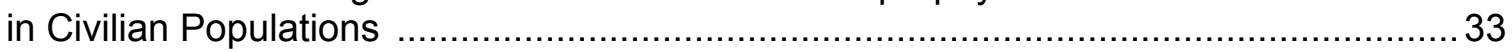

Phenomena Affecting Adherence to Malaria Chemoprophylaxis Medications

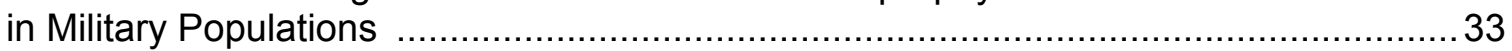

Other Military Health Behaviors: Weapon Safety and Condom Use ............................34

Why Knowledge, Attitudes, and Practices? ................................................................ 36

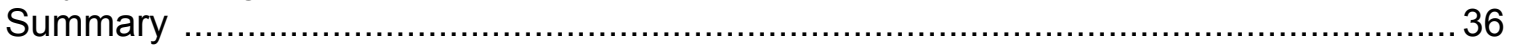

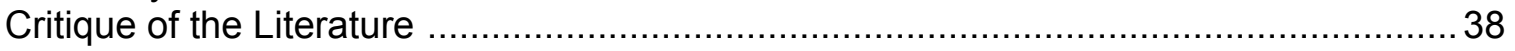

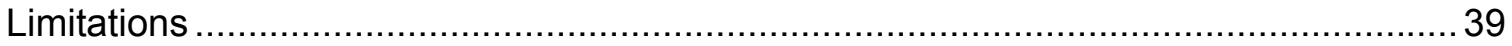




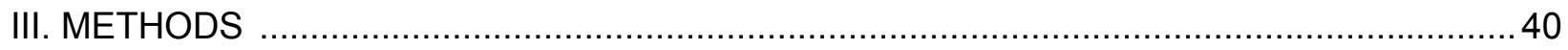

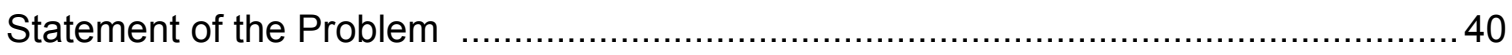

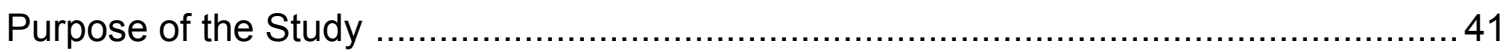

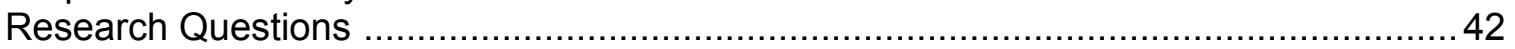

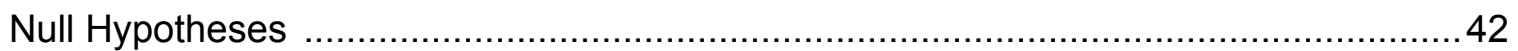

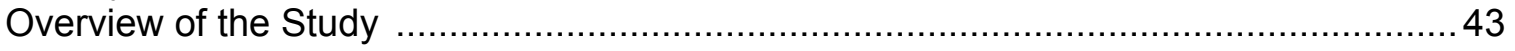

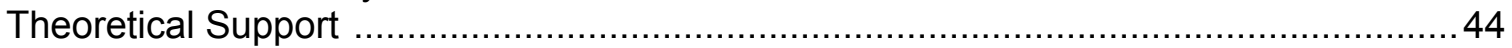

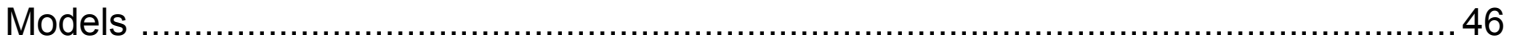

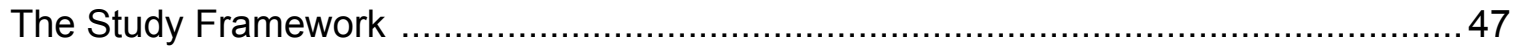

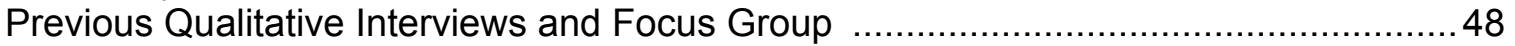

Instruments and Measures ......................................................................... 50

Pre-Construction of the Instrument ......................................................... 51

The Final Survey Instrument ………................................................. 52

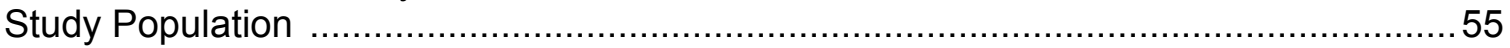

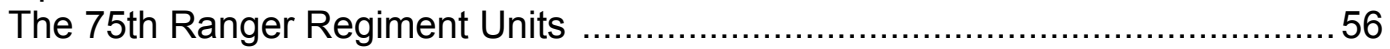

Similarities within the Ranger Population ................................................. 58

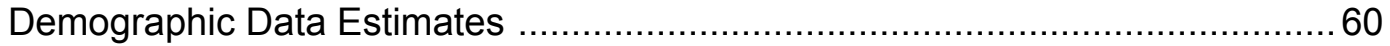

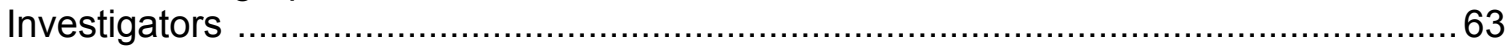

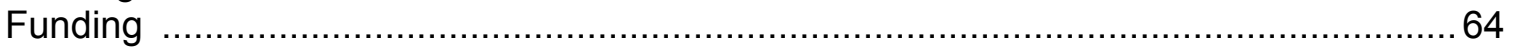

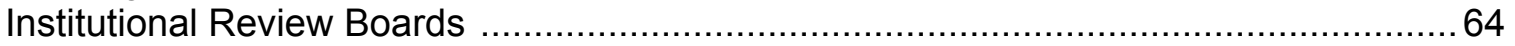

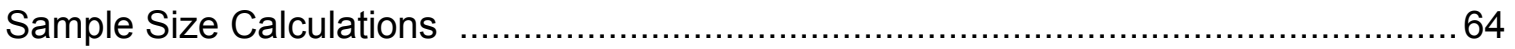

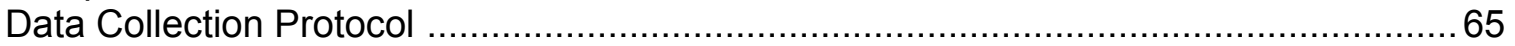

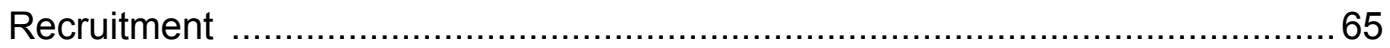

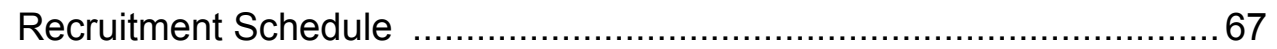

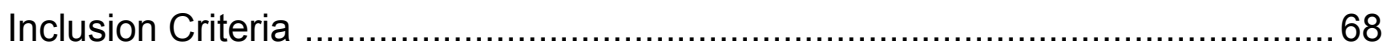

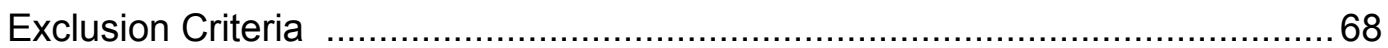

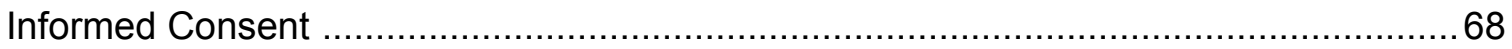

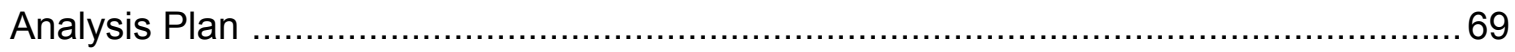

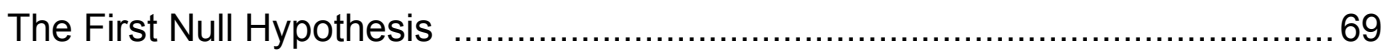

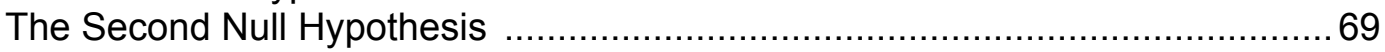

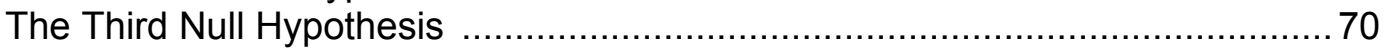

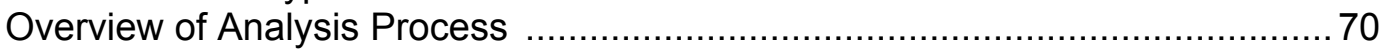

Organizing the Database for Analysis ……………….......................... 70

The Dependent Variables ............................................................ 72

Independent Variables .............................................................. 74

Selecting Variables for Analysis ....................................................... 74

Estimates of Internal Consistency Reliability Analysis of the

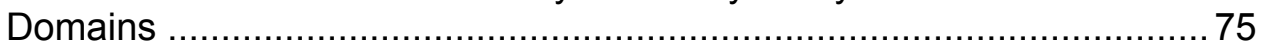

Transforming and Cleaning Variables for Analysis …………………..... 76 Identification of the Newly Transformed Variables ......................78

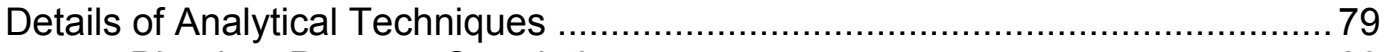

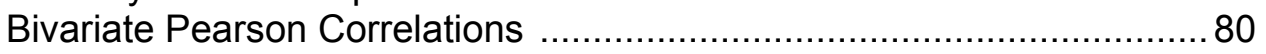

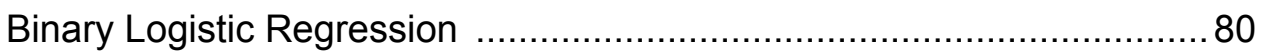

Forward Stepwise, Conditional Method ……………................ 80

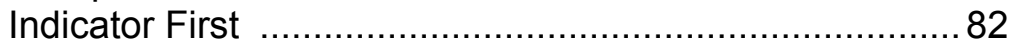

The Moderated Logistic Regression …………….............................. 82

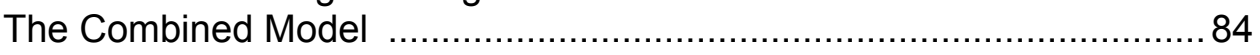

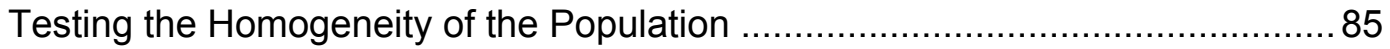




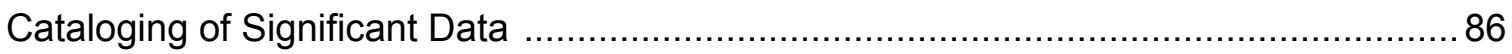

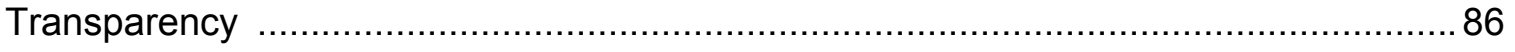

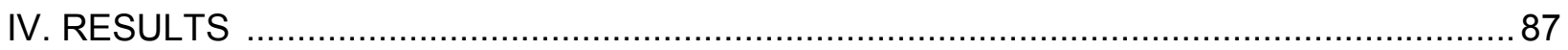

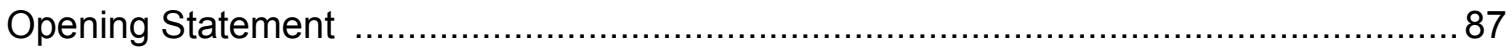

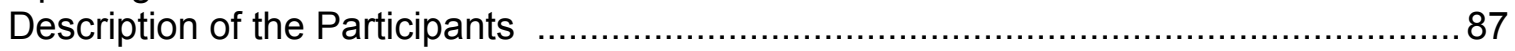

Levels of Adherence and Intentions to Adhere ………...........................................93

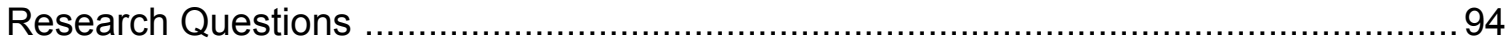

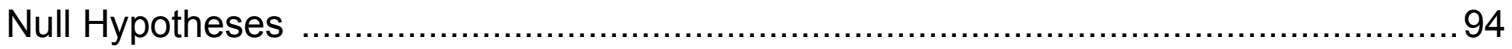

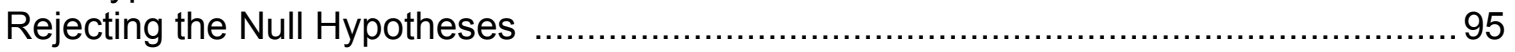

The First Null Hypothesis ...................................................................... 95

Significant Bivariate Pearson Correlation Coefficients: Malaria

Chemoprophylaxis Medications …………………..................95

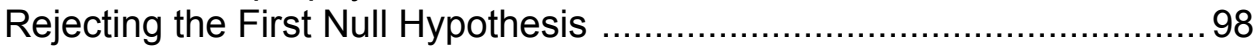

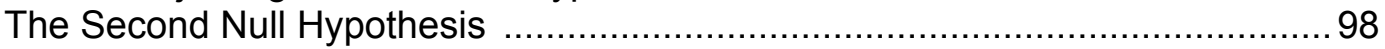

Combined Models .......................................................................

Full Combined Model ........................................................ 99

Validation of Odds Ratios ………………………...............100

Ever/Never Combined Model .................................................101

Rejecting the Second Null Hypothesis .............................................103

The Third Null Hypothesis ..................................................................... 103

Moderator Interactions with the Outcome Variable: Intend to Take

Meds the Next Time ..................................................................... 103

Moderator Interactions with the Outcome Variable: Take Meds as

Directed .................................................................................. 103

Interpretations of the Four (4) Moderator Interaction Models ...106

Results of the Four (4) Moderator Interactions ........................ 112

Rejecting the Third Null Hypothesis ................................................112

Illustration of Knowledge Relative to the Behaviors ……………………………... 112

Gradable Knowledge Results ..........................................................113

One-Way Analysis of Variance ……………………....................................113

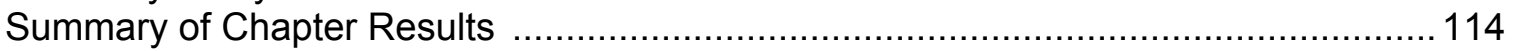

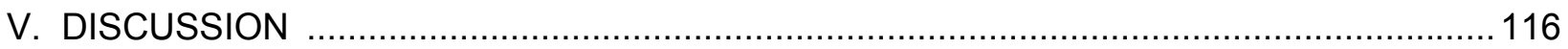

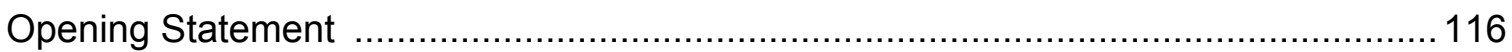

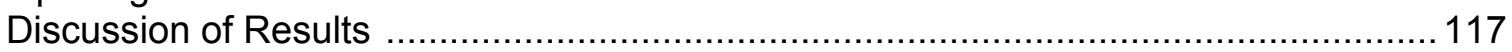

Description of the Participants ............................................................... 117

Levels of Adherence and Intentions to Adhere ........................................118

The Restated Research Questions ……........................................................122

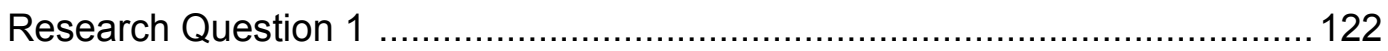

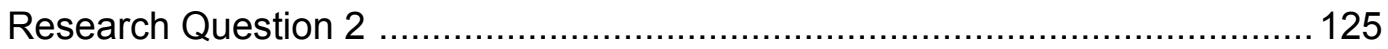

The Full Combined Model ..........................................................125

The Ever/Never Combined Model ..................................................126

Theoretical Explanations for the Combined Model Results ......128

References Within the Literature ……………………........... 129

Research Question 3 .................................................................. 130

Theoretical Explanations for the Moderated Interactions .......... 134

References Within the Literature ......................................... 135 
Illustration of Knowledge Relative to Adherence Levels ...................................... 136

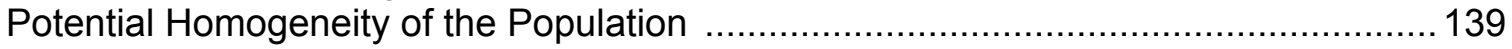

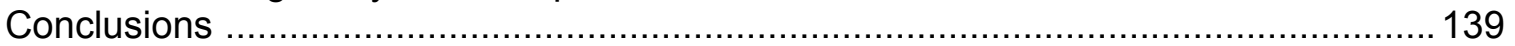

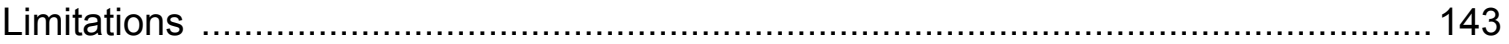

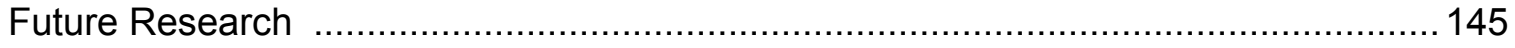

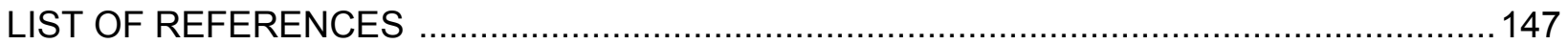

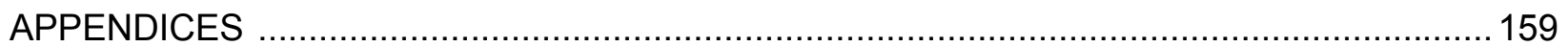

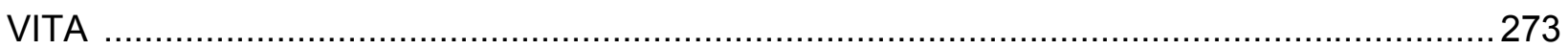




\section{LIST OF TABLES}

TABLE

PAGE

3.1. Cronbach's Alpha Coefficients for KAP and Environmental Domain Measures..................76

4.1. Significant Bivariate Correlation Coefficients with Dependent Variable, Take Meds as Directed

4.2. Significant Bivariate Correlation Coefficients with Dependent Variable, Intend to Take Meds as Directed

4.3. Final Full Combined Logistic Regression Model, Predicting the Intention to Take the Medications the Next Time

4.4. Final Ever/Never Combined Logistic Regression Model, Predicting the Intention to Take the Medications the Next Time 102

4.5. Moderator Variables and their Significant Interaction Terms, Relative to the Dependent Variable Take Meds as Directed...... 


\section{LIST OF FIGURES}

FIGURE

PAGE

1.1. NCPIE, Strategies for Improving Patient Adherence ..............................................30

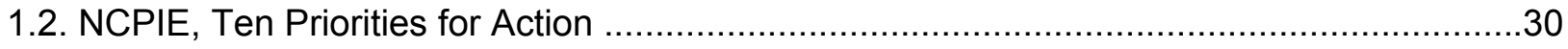

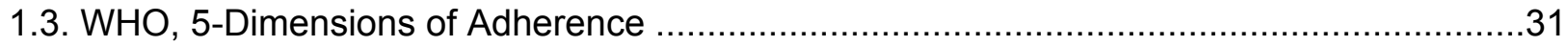

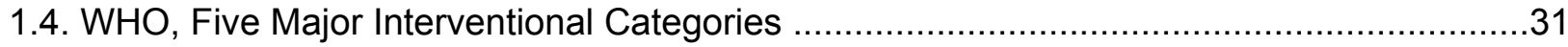

1.5. Osterberg and Blaschke, Four Major Interventional Categories ..................................32

1.6. Cleemput, et al., Five Major Interventional Categories .................................................32

3.1. Theoretical Diagram Model: Mediator Effect and Moderator Interaction .........................47

4.1. Estimated Proportion of Ideal Participation vs. Actual Study Participants from each Ranger

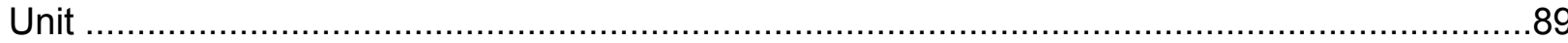

4.2. Percent Estimate of Ranger Population Age vs. Actual Study Participants .....................91

4.3. Percent Estimate of Ranger Population Rank Structure vs. Actual Study Participants .......92

4.4. Estimated Percent Ethnicity of Ranger Population vs. Actual Percent of Study Participants 


\section{LIST OF ACRONYMS AND ABBREVIATIONS}

Alpha

$\alpha$

Analysis of Variance

ANOVA

Antiretroviral therapy

ART

Centers for Disease Control and Prevention

CDC

Chemoprophylaxis

Chemo

Chief Warrant Officer

CW

Commissioned Officer

$\mathrm{O}$

Confidence Interval

$\mathrm{Cl}$

Congressional Research Service

CRS

Data Coordinating and Analysis Center

DCAC

Department of Defense

DoD

Department of the Army

DA

Directly Observed Therapy

DOT

Division of Human Subjects Protection

DHSP

Doxycycline

Doxy

Glucose-6-phosphate dehydrogenase

G6PD

General Accounting Office

GAO

General Services Administration

GSA

Florida International University

FIU

Health Belief Model

HBM

Health Care Provider

$\mathrm{HCP}$

Highly Active Antiretroviral Therapy

HAART

Knowledge, Attitudes, and Practices

KAP

Least-significant-difference

LSD 
Long-lasting Insecticidal Nets

LLINS

Medications

Meds

Military HIV Research Program

MHRP

Military Infectious Disease Research Program

MIDRP

Military Occupational Skills

MOS

Modified Version of Directly Observed Therapy

m-DOT

National Council on Patient Information and Education

NCPIE

Non-Commissioned Officer

NCO

Pre-Special Operations Combat Medical Course

PSOCM

Primary Investigator

$\mathrm{Pl}$

Ranger Assessment and Selection Program

RASP

Regimental Headquarters

$\mathrm{RHQ}$

Regimental Special Troops Battalion

RSTB

Secure File Transfer

SFT

Significant

Sig

Small Unit Ranger Tactics

SURT

Social Cognitive Theory

SCT

Special Operations Force

SOF

Standard Error

SE

Theory of Reasoned Action/Theory of Planned Behavior

TRA/TPB

United Nations

UN

United Nations Development Programme

UNDP

United States

U.S. 
United States Army, Headquarters

USA HQ

United States Army Combat Readiness/Safety Center

US Army CRSC

United States Army Forces Central Command

USARCENT

United States Army Public Health Command

USAPHC

United States Army Special Operations Command

USASOC

United States Department of Health and Human Services

DHHS

United States Southern Command

USSOUTHCOM

Walter Reed Army Institute of Research

WRAIR

With Dropped Response Entries

wDs

World Bank

WB

World Health Organization

WHO 


\section{CHAPTER I}

\section{INTRODUCTION}

\section{Opening Statement}

Malaria is a parasitic infection of the Plasmodium genus with over 100 species, of which only five threaten humans (McPhee et al., 2007; Rowe, Claessens, Corrigan, \& Arman, 2009; Strickland, 2000; Taoufiq et al., 2008). The remaining species tend to infect only monkeys, birds, rodents, and some reptiles (Ayala, S. \& Spain, J., 1976; Dasgupta, 1965; Hewitt, 1941).

Reports of human malaria date back to 2000 B.C. and it was once considered the greatest human killer of all infectious diseases (Cockburn, 1963). Although malaria is not the greatest killer today, it is endemic to 106 countries and threatens about 3.3 billion people (WHO, 2010). In 2009, approximately 225 million cases of malaria and 781,000 deaths were reported (WHO, 2010). Malaria is responsible for huge economic burdens, which costs Africa alone an estimated \$12 billion each year (Breman et al., 2006; Sarbib, Nankani, \& Patel, 2006; World Bank, 2005).

Travelers including military personnel venturing to malaria-endemic regions risk contracting malaria. This risk can be greatly reduced with appropriate prevention measures, of which malaria chemoprophylaxis medications are an important component (CDC, 2009b; Cook, \& Zumla, 2008; WHO, 2008b). Scholarly articles report that despite having these preventive medications available, both civilian (Ben-Ami et al., 2005; Landry, Iorillo, Darioli, Burnier, \& Genton, 2006) and military travelers (Hagan, Marcos, \& Steinberg, 2010; Kotwal et al., 2005) often choose not to take them properly.

\section{Statement of the Problem}

The United States (U.S.) military reported 423 cases of malaria between 2003 and 2005 (Ciminera \& Brundage, 2007; Kotwal et al., 2005). There were at least 12 cases in Haiti during the first two months of the 2010 earthquake recovery efforts, and new cases of malaria continue 
to be documented in U.S. military medical and public health reports (Robson, 2010; USAPHC, 2010; Whitman et al., 2010). Each U.S. military case of malaria might have been avoided had they properly taken their malaria chemoprophylaxis medications (Garges, 2007; Kotwal et al., 2005; LaFon, \& Scoville, 2006; Robson, 2010; Whitman et al, 2010).

The former Surgeon General, C. Everett Koop, is commonly quoted as saying, "Drugs don't work if patients don't take them," which is especially true with malaria chemoprophylaxis medications (Abraham et al., 1999; Ben-Ami et al., 2005; Dupouy-Camet et al., 2003). Due to their narrow therapeutic range and relative half-life, these preventive chemoprophylaxis medications require $100 \%$ adherence to maintain efficacious levels (Cook, \& Zumla, 2008; GlaxoSmithKline, 2010; Sanofi-aventis, 2008; Sanofi-Synthelabo, 2007; STAT RX, 2010; Strickland, 2000; West-ward Pharm, 2010). Despite this requirement to maintain $100 \%$ adherence, these medications are notoriously very poorly adhered to and the reasons are not well understood, especially in military populations.

The many factors, barriers, and predictors affecting general medical adherence are often complex and vast (Albert et al., 2009; Amico et al., 2007; Bosworth et al., 2005; Cleemput et al., 2002; DiMatteo, Hays, \& Sherbourne, 1992; DiMatteo, 2004; WHO, 2003). Adherence becomes even more complex during military training and combat operations that may detract from any medical therapy, including the strict adherence required of malaria chemoprophylaxis medications (Ciminera \& Brundage, 2007; Garges, 2007; Kotwal et al., 2005; LaFon, \& Scoville, 2006; Resseguier et al., 2010; Whitman et al, 2010). Therefore, it is important to better understand how to improve this behavior in military populations.

The U.S. Army Special Operations Command (USASOC) is charged with training and some of this nation's most elite fighting forces, one of these is the U.S. Army Ranger: referred to from this point on as Rangers. Rangers are known to have contracted malaria because they did 
not follow preventive measures as recommended, specifically by not taking their preventive malaria chemoprophylaxis medications as directed (Kotwal et al., 2005; USPHC, 2010

Rangers operate in relative secrecy. Their deployment and training cycles take them to some of the most austere and dangerous regions of the world, many of which are endemic with malaria (USASOC, 2011a, 2011b; WHO, 2010). These active training and deployment cycles, combined with their relative secrecy, have resulted in very limited access to these populations for the purpose of any type of scientific observation.

The lack of access to this Ranger population has created a general dearth of information on a variety of topics; particularly health behaviors and the knowledge, attitudes, and practices (KAPs) associated with them. This study will help to improve this knowledge gap.

The USASOC understands that malaria prevention measures are important and recognizes the relevant KAPs are understudied, particularly in their Ranger population assigned to the 75th Ranger Regiment.

In an effort to improve public health support and improve future health outcomes in the Ranger population, the USASOC and the 75th Ranger Regiment Commander have granted access to the Ranger population for this KAP study and therefore, the Rangers of the 75th Ranger regiment are the study population of this research.

\section{Purpose of the Study}

The reasons US military personnel choose not to follow medical guidance to take preventive malaria chemoprophylaxis medications properly are not clear and no studies exist that thoroughly examine the relevant KAPs in this population. This exploratory study will fill this gap in the current knowledge and also inform future interventions aimed at improving this behavior throughout the military. I plan to utilize the knowledge gained from this study to begin planning future research and interventions with the U.S. Army Public Health Command immediately after my graduation in the fall of 2012 . 


\section{Research Questions}

This study focused on answering three research questions:

1. What relationship, if any, exists between the current KAP domains, environmental domains, and behavioral outcomes?

2. Can any of the behavioral domain variables predict behavioral intentions, as a proxy for behavior change? Behavioral intention is the best predictor of behavioral change (DiClemente, Crosby, \& Kegler, 2009; Edberg, 2007).

3. Do variables within the environmental domains of behavioral education, behavior related training, behavior related media, or military command support moderate the relationship between any KAP domain variables? Personal characteristics are included in all the KAP domain variables.

\section{Null Hypotheses}

The three research questions were subsequently transformed into the following Null Hypotheses:

H10: No relationship exists between the current KAP domains, environmental domains, and behavioral outcomes.

$H 2_{0}$ : The current KAP domain variables cannot predict behavioral intentions, as a proxy for behavior change.

H3o: Command Support domain variables do not moderate the relationship between variables within the KAP domains of perceived susceptibility and risk, behavior related practices, interpersonal communications, and the behavior outcomes. 


\section{CHAPTER II}

\section{LITERATURE REVIEW}

A dearth of information exists concerning the KAPs associated with malaria prevention in any U.S. military population; therefore, the information supporting this study is predicated on the available literature relative to multiple subjects, including: malaria, preventive malaria chemoprophylaxis medications, the abundant literature supporting adherence to general medical therapies, the limited literature supporting adherence to preventive malaria chemoprophylaxis medications, malaria outbreak and prevention studies in both civilian and military populations, and KAP frameworks.

\section{The Roadmap: A Guide through the Literature Review}

This is an exploratory study on a subject that is not well studied, in a population that is rarely made available or accessible to researchers studying health behaviors. Additionally, there is no one source of available literature that encompasses the breadth or depth of information required to grasp the knowledge necessary effectively inform a study of this kind. Therefore, this literature review examines a multitude of subjects and areas within those subjects. To better prepare the reader and inform him/her in advance of what they are to expect here, this roadmap has been created.

The review will begin with an overview of the disease malaria. It will then review preventive measures that include the chemoprophylaxis medications used to prevent the disease. These chemoprophylaxis medications will be discussed in brief detail to leave very little question as to their efficacy or necessity.

Next, the review will examine the terms adherence and compliance and why adherence is the most appropriate term to utilize relative to military populations. This may seem elementary or a mute point to some but can be somewhat complex given the authoritative nature of the 
military. Following this section, a brief examination of quantifying and measuring adherence is examined.

The review will then examine the limited body of literature supporting adherence to malaria chemoprophylaxis medications in both military and civilian populations: the former representing a greater dearth of information than the latter. Due to this relative dearth of information concerning adherence to malaria chemoprophylaxis medications, the review will then examine the larger corpus of literature and information supporting adherence to general medical therapies: the cornerstone of this literature review.

Finally, the review will examine strategies to improve adherence and adherence related issues that affect both general medical therapies and malaria chemoprophylaxis medications. The review then concludes with a brief examination of KAP frameworks, a brief summary, and a limited critique of the literature.

\section{Background on Malaria and Malaria Epidemiologic Data}

Malaria has plagued mankind since the earliest medical records dating back to around 2000 B.C (Cockburn, 1963). Human malaria is believed to have originated in Africa, possibly evolving tens of millions of years ago from intestinal parasites which mutated and spread from blood eating invertebrates (e.g. leaches) to eventually infect old world monkeys, then humans (Cockburn, 1963). In the 1930s and 40s, malaria was considered the greatest killer of mankind of all infectious diseases (Cockburn, 1963).

Though malaria is not the greatest killer today, it is endemic to 106 countries and threatens about 3.3 billion people (WHO, 2010). In 2009, approximately 225 million cases of malaria and 781,000 deaths were reported (WHO, 2010). Most deaths (85\%) are to children under 5 years old (WHO, 2009). This is equivalent to 1 child dying of malaria about every 30 seconds (Sarbib, Nankani, \& Patel, 2006; UNDP, 2010). 
Malaria is a parasitic infection of the Plasmodium genus with over 100 species, of which only five threaten humans (McPhee et al., 2007; Rowe, Claessens, Corrigan, \& Arman, 2009; Taoufiq et al., 2008; Strickland, 2000). Four species are common threats to humans: Plasmodium vivax (P. vivax), Plasmodium ovale (P. ovale), Plasmodium malariae (P. malariae), and Plasmodium falciparum (P. falciparum) (McPhee et al., 2007). The fifth species, Plasmodium knowlesi, usually only infects certain primates but may rarely infect humans (Bronner, Divis, Farnert, \& Singh, 2009; McPhee et al., 2007; Singh et al., 2004). The remaining species of malaria infect animals including monkeys, birds, rodents, and even some reptiles (Ayala, S. \& Spain, J., 1976; Dasgupta, 1965; Hewitt, 1941).

Malaria strikes with a rapid onset of severe fevers and chills, followed by symptoms that may include painful myalgias, headache, nausea, vomiting, and diarrhea (McPhee et al., 2007). It is transmitted to man through the bite of the female Anopheles mosquito which introduces the parasitic infection to infect the liver and red blood cells causing severe illness, anemia, and damage to other tissues that may lead to death (McPhee et al., 2007; Rowe, Claessens, Corrigan \& Arman, 2009; Taoufiq et al., 2008). Death can occur in as fast as 24-hours after symptom onset.

The global burden of malaria is tremendous and is not only measured directly in human sickness and death, but also through indirect measures that affect the global economy. In the highly endemic countries of Africa, malaria contributes to a slow economic growth. From 1965 to 1990 malaria endemic countries of Africa showed a average annual financial growth of only $0.4 \%$, compared to malaria free country's that grew an average of $2.3 \%$ annually (Sarbib, Nankani, \& Patel, 2006; World Bank, 2005). There are estimates that malaria singularly impacts the economies of highly endemic countries with an overall annual GDP loss of 1.3\% (Breman, 2006; World Bank, 2005). Malaria costs Africa an estimated $\$ 12$ billion each year (World Bank, 2005). 
Many areas other than Africa are also affected by malaria, including endemic regions of South and Central America, Haiti and the Dominican Republic, the Eastern Mediterranean, parts of Europe, South-East Asia, and the Western Pacific region (WHO, 2008b). Though malaria has a global presence, the impacts are in Africa (WB, 2005; WHO, 2008b).

\section{Non-Medical Malaria Preventive Measures}

Travelers who venture into malaria endemic areas can protect themselves by taking non-medical preventive measures like sleeping indoors or under mosquito nets, wearing protective clothing, and applying effective repellants (CDC, 2009a; McPhee et al., 2007).

Current malaria intervention and prevention measures used around the world for nontravelers who reside in malaria endemic regions include providing long-lasting insecticidal nets (LLINS), supplying effective treatment medications, providing various preventive educational programs, conducting periodic indoor spraying of at risk homes, and expanding and/or improving research (WHO, 2009).

\section{Malaria Chemoprophylaxis Medications, General Medical Considerations}

Malaria chemoprophylaxis is the prevention of malaria attacks with specific medications that are prescribed based on the traveler's specific risk of infection and taken at regular intervals prior to exposure, during exposure, and for specified periods post exposure to malaria (McPhee et al., 2007). These medications do not prevent mosquito bites, but destroy or prevent the maturation of malaria in human blood and tissue (Gilbert et al., 2007; McPhee et al., 2007). Commonly prescribed malaria chemoprophylaxis medications available in the United States include Chloroquine, Mefloquine, Atrovaquone + Proquanil (Malarone), Doxycycline, and Primaquine (CDC, 2009a; Gilbert et al., 2007; McPhee et al., 2007).

Though malaria chemoprophylaxis medications have been proven to provide significant protection, they are not $100 \%$ effective at preventing all malaria attacks. Some infections may occur even though travelers are adherent with them and take them as directed (McPhee et al., 
2007). Nevertheless, non-adherent travelers put themselves at a much greater risk than adherent travelers (Kimura, Kawakami, Hashimoto, \& Hamada, 2006; Shanks et al., 2005).

Malaria may also become resistant to certain chemoprophylaxis medications. This is a key factor to consider when determining which medications are most appropriate to prescribe for travelers. Resistance categories are generally divided first into Chloroquine susceptible and Chloroquine resistant malaria; then further into regions resistant to other medications such as Malarone and Mefloquine (CDC, 2009a; Gilbert et al., 2007; McPhee et al., 2007; WHO, 2008a). There are no strains of malaria that are known to be resistant to Doxycycline (Epocrates, 2009; Gilbert et al., 2007; McPhee et al., 2007).

Other important factors to consider when using or prescribing malaria chemoprophylaxis medications are: length of stay in the endemic region, time until departure to the region, types of malaria in the region, traveler age, traveler pregnancy status, individual contraindications, and potential adverse effects of the medications (CDC, 2009a; Gilbert et al., 2007; McPhee et al. 2007).

\section{Specific Malaria Chemoprophylaxis Medications}

\section{Chloroquine}

Chloroquine is considered the drug of choice for malaria chemoprophylaxis and is effective when resistance is not present (CDC, 2009a; Gilbert et al., 2007; McPhee et al., 2007). Unfortunately, many strains of malaria around the world are resistant to Chloroquine. It is generally considered safe and well tolerated by most travelers. Some adverse effects may include gastrointestinal symptoms, mild headache, pruritus, and dizziness (McPhee et al., 2007). Though rare, other more severe reactions may present, especially to Glucose-6phosphate dehydrogenase (G6PD) deficient travelers (McPhee et al., 2007). Chloroquine is one of only two medications available in the United States (Mefloquine is also considered safe) that is considered safe during pregnancy (CDC, 2009a; Gilbert et al., 2007; McPhee et al., 2007). 


\section{Mefloquine}

Mefloquine is a commonly used malaria chemoprophylaxis medication. Mefloquine can be used in many Chloroquine resistant regions of the world; though Mefloquine resistant strains of malaria are found in some regions (CDC, 2009a; Gilbert et al., 2007; McPhee et al., 2007).

Mefloquine has also been found to be effective and requires only once weekly dosing (CDC, 2009a; Gilbert et al., 2007; McPhee et al., 2007, Ohrt et al.et al. 1997). Despite the advantage of once weekly dosing and efficacy, Mefloquine has many contraindications and may be associated with the onset of severe neuropsychiatric events such as anxiety, depression, mood swings, insomnia and nightmares (Aarnoudse et al., 2006; Gilbert et al., 2007; McPhee et al., 2007). Other adverse effects may include gastrointestinal symptoms, headache, dizziness, syncope, and extrasystoles (McPhee et al., 2007). Major contraindications to Mefloquine include current or past history of anxiety, depression, psychosis, schizophrenia, other major psychiatric disorders, or a history of seizures (CDC, 2009a; McPhee et al., 2007; Tarascon, 2007).

\section{Malarone}

Malarone is another preventive malaria chemoprophylaxis medication. Malarone is effective against all types of malaria, as well as both the blood and liver stages of Plasmodium Falciparum malaria (McPhee et al, 2007) and is considered the first line medication in the Sanford Guide to Antimicrobial Therapy (2007). Few strains of malaria are known to be resistant to Malarone and it can be used in many areas with Chloroquine resistant malaria (CDC, 2009a; Gilbert et al., 2007; McPhee et al., 2007). Despite its efficacy, there are some areas in Africa that have developed resistance to Malarone (CDC, 2009a; McPhee, 2007).

Malarone must be taken daily and considered by many to be better tolerated than Chloroquine and have much fewer adverse effects than Mefloquine (Gilbert et al., 2007; McPhee et al., 2007). Adverse effects associated with Malarone may include gastrointestinal 
symptoms, headache, rash, dizziness, and mild reversible elevations of liver aminotransferases (McPhee et al., 2007). It is contraindicated for use during pregnancy (McPhee et al., 2007).

A meta-analysis and systematic review of 10 studies by Nakato, Vivancos and Hunter (2007); found Malarone to be safe, well tolerated and highly effective against all types of nonresistant malaria: efficacy was above placebo at $95.8 \%(95 \% \mathrm{Cl}=91.5-97.9)$.

\section{Doxycycline}

Doxycycline is the only preventive chemoprophylaxis medication that is both effective against all types of malaria and is indicated for chemoprophylaxis worldwide with no known areas of resistance (Epocrates, 2009; Gilbert et al., 2007; McPhee et al., 2007). No studies or literature have been identified that conclude malaria to be resistant to Doxycycline. It is generally well tolerated with only a few, usually mild, adverse effects which include gastritis, candidal vaginitis, and photosensitivity (Gilbert et al., 2007; McPhee et al., 2007).

Doxycycline must be taken daily and is usually recommended to be taken with a meal and copious amounts of water (McPhee et al., 2007). It is generally considered safe for nonpregnant adults and children above the age of 8 years old (CDC, 2009a; Gilbert et al., 2007; McPhee et al., 2007). Contraindications include pregnancy, less than eight years of age, allergic reactions, and intolerability to any adverse effects (Gilbert et al., 2007; McPhee et al., 2007).

Ohrt et al. (1997) conducted a clinical trial studying the efficacy of both Mefloquine and Doxycycline. This 13-week, randomized, double-blind, placebo-controlled trial of 204 Indonesian soldiers showed both Mefloquine and Doxycycline to be similarly efficacious preventive malaria chemoprophylaxis agents, above placebo. Mefloquine was found to have $100 \%$ efficacy $(\mathrm{Cl}=$ $96 \%-100 \%)$ and Doxycycline having $99 \%$ efficacy (95\% Cl $=94 \%-100 \%)$ for the types of malaria endemic to the study areas of Indonesia. Although the study was conducted in 1997, the CDC Yellow Book (2009a) continues to recommend these medications for that region. 


\section{Primaquine}

Primaquine is the final preventive malaria chemoprophylaxis medication commonly used in the United States. Primaquine is utilized as a terminal chemoprophylaxis measure when travelers have been exposed to Plasmodium Vivax, Plasmodium Ovale, or Plasmodium Falciparum malaria (Gilbert et al., 2007; McPhee et al., 2007).

Terminal chemoprophylaxis is an additional daily dose of Primaquine, which is usually added to the final 2 weeks of the primary chemoprophylaxis regimen. This is required to better insure eradication of any liver stage malaria parasites that may remain in the body despite the regular use of normal preventive chemoprophylaxis medications, which may not effectively reach these parasites and prevent malaria alone (McPhee et al., 2007).

Primaquine is usually very well tolerated. Adverse effects of Primaquine include gastrointestinal disturbances, headache, dizziness and neutropenia (McPhee et al., 2007). Primaquine should be used with caution in patients with G6PD deficiency and is not safe for use during pregnancy (CDC, 2009a; Gilbert et al., 2007; McPhee et al., 2007).

\section{Defining Adherence and Compliance}

Adherence is defined in Dorland's Medical Dictionary as "the act or quality of sticking to something" (1995, p.16). The World Health Organization (WHO) defines it as , "the extent to which a person's behavior - taking medication, following a diet, and/or executing lifestyle changes, corresponds with agreed recommendations from a health care provider" (2003, p. 18). The term adherence is the preferred term, compared to the term compliance, when considering medical therapies (WHO, 2003); nevertheless, the terms are often used interchangeably. The preponderance of recent literature has adopted the term adherence (NCPIE, 2007; Osterberg \& Blaschke, 2005; RAND, 2009; WHO, 2008a).

Non-adherence to medications can be defined as any one, or combination, of the following: starting a medication late, taking an inappropriate dose, taking a medication at 
improper intervals, stopping earlier than recommended, forgetting to take a medication, not understanding the regimen, failing to refill a prescription, and/or taking a holiday from one or more medications (Klein, Wustrack, \& Schwartz, 2006; Landry et al., 2006; WHO, 2003; Martin, Williams, Haskard, \& DiMatteo, 2005).

Compliance is defined by Merriam-Webster (2010) as, "the act or process of complying to a desire, demand, proposal, or regimen, or to coercion; conformity in fulfilling official requirements; a disposition to yield to others."

The WHO considers compliance an inappropriate term when used in conjunction with medical therapies because of its tendency to be associated with blame on the patient, its inability to justly identify with the many levels of care associated with medical therapies, and its inability to capture the active role the patient must take in a therapy (WHO, 2003). Therefore, in 2003, the WHO adopted adherence as the most appropriate term when applied to medical therapies. It is important to note that neither adherence nor compliance are perfect terms and are often used interchangeably (NCPIE, 2007; Osterberg \& Blaschke, 2005; RAND, 2009; WHO, 2008a).

\section{The Most Appropriate Term in Military Populations: Adherence vs. Compliance}

Since the ultimate focus of the knowledge gained from this research will be applied to military populations, it is important to distinguish between the terms adherence and compliance to determine which is most appropriate for this population.

During times of war and imminent danger, military members may be ordered to comply with medical therapies. This order has been used multiple times as an attempt to force service members to take malaria chemoprophylaxis medications (USSOUTHCOM, 2004; USARCENT, 2006). If this order action is taken, it is initiated by the commanding officer, not the healthcare provider. Nevertheless, the military healthcare provider maintains the ultimate medical decision 
supporting this action; which is made only after carefully considering the impact of the medication and the needs of the patient (DA, 2009; DA, 2010).

This being said, there is no true difference between civilian and military medical treatment. Patients still have a choice to participate in their treatment, or not. Their health, safety, and dignity remain the primary concerns of all military healthcare providers. The patient and provider will always be in agreement before any medication is prescribed; therefore, it stands to reason that adherence is the most appropriate term to describe the patient's correspondence to "...agreed recommendations from the healthcare provider...," (WHO, 2003, p. 18) in military populations as well as civilian populations.

\section{Is Compliance Ever the Proper Term?}

Generally, in the more recent literature, compliance is considered appropriate for use when it is referring to rules and regulations that insure processes are conducted properly, and when patient actions or behaviors are not the focus of the word's use (Bosworth, Olsen, \& Oddone,2005; NCPIE, 2007; WHO, 2003). The term is often used when 1) health care providers are required to follow specific or accepted treatment guidelines (Albert et al., 2009; Pierson, 2010), 2) when hospital administrative procedures are to be followed in a specific manner (DA, 2010; "Don't get complacent," 2010; "ED makes," 2010), and 3) during the evaluation and/or monitoring of research procedures and protocols ("Compliance alliance," 2010; "Going pro," 2010; "Institution's reviews," 2010; "Six Steps," 2010). There are undoubtedly other appropriate uses for compliance, and policy makers or evaluators may choose not to use the term compliance and select the less blame implying term of adherence during administrative processes and/or critical professional evaluations (DA, 2010; "Lack of adherence," 2010).

\section{Quantifying Adherence}

Quantifications of adherence levels are common in the corpus of available literature.

What is not common is a gold-standard that defines what constitutes non-adherence (Cleemput 
et al., 2002; Hays et al., 1994; Osterberg \& Blaschke, 2005; WHO, 2003). Non-adherence can range from any level less than $80 \%$ to $95 \%$; depending on the disease process (Osterberg \& Blaschke, 2005). Often non-adherence is judged empirically, not to exceed any level considered to be progressively detrimental to patient health (WHO, 2003). Concerning malaria chemoprophylaxis medications, this level is anything less than $100 \%$ adherence (Cook, \& Zumla, 2008; GlaxoSmithKline, 2010; Sanofi-aventis, 2008; STAT RX, 2010; Strickland, 2000).

\section{Measuring Adherence Levels}

Methods available for measuring adherence to medical therapies include: self report, electronic monitoring devices, pill counts, measuring body fluid drug concentrations, physician reports, observing biological markers, and direct observation (Abraham, Clift, \& Grabowski, 1999; Ben-Ami, Seigman-Igra, Anis, Brook, Pitlik, Dan., 2005; Cleemput et al., 2002; DupouyCamet, Yera, \& Tourte-Schaeffer et al., 2003; Osterberg \& Blaschke, 2005; Landry, lorillo, Darioli, Burnier, \& Genton 2006; Martin et al., 2005; RAND, 2009; Whitman et al., 2010; WHO, 2003). Despite the many available methods, only a few have been used in the study of adherence to malaria chemoprophylaxis medications. The most predominant method in this case is self report (Abraham et al., 1999; Ben-Ami et al., 2005; Cleemput et al., 2002; DupouyCamet et al., 2003; Garges, 200; Hays, n.d.; LaFon, \& Scoville, 2006; Martin et al., 2005; Resseguier et al., 2010; Whitman et al, 2010; WHO, 2003). In fact, self report was used in every malaria chemoprophylaxis adherence study identified in the literature; occasionally in combination with other methods. Two studies validated the self report by comparing serum drug concentrations of chemoprophylaxis medications (Ollivier et al, 2008; Whitman et al, 2010), and one study used electronic monitors on pillboxes, in addition to self report (Landry et al., 2006).

\section{Civilian Adherence to Preventive Malaria Chemoprophylaxis Medications}

The literature concerning adherence to malaria chemoprophylaxis medications in civilian populations is more complete than the literature supporting military populations; nevertheless, 
both populations report similar levels of non-adherence to these medications. This section will report the general findings in the literature relative to civilian populations and their inadequate levels of adherence (Abraham et al., 1999; Baudon, Michel, Mynard, Keundjian, \& Martet, 1997; Ben-Ami et al., 2005; Berg \& Visser, 2007; Dupouy-Camet et al., 2003; Hymel, \& Yang, 2008; Landry et al., 2006; Okeck et al., 2009; Shanks, \& Edstein, 2005).

This review does not claim to include every study conducted; nevertheless, each study found in the literature reported inadequate levels of adherence to malaria chemoprophylaxis medications. There were no studies identified that reported trends in civilian populations that remotely approached the $100 \%$ adherence required to maintain protective serum levels.

To give a broad perspective of these civilian studies, three studies of travelers from around the world have been selected to characterize the levels of adherence to malaria chemoprophylaxis medications found in civilian populations. The first study surveyed 169 Israeli travelers who contracted malaria, (Ben-Ami et al. 2005). Of these 169 travelers, only 17 travelers or $10 \%$ reported that they had taken their malaria chemoprophylaxis medications exactly as directed. Another study of 81 Swiss travelers who ventured into Sub-Sahara Africa, who were also monitored with electronic pillboxes that reported the exact times and doses they took of their malaria chemoprophylaxis medications, revealed that only $32 \%$ of those travelers took their medications on time and as directed (Landry et al., 2006). A third study found that only $13 \%$ of a study population of 160 Japanese travelers, who had traveled to various malaria endemic areas, took any amount of malaria chemoprophylaxis medications during their travels into these endemic areas (Kimura et al., 2006).

\section{Military Adherence to Preventive Malaria Chemoprophylaxis Medications}

There is a relative dearth of behavioral research on military populations associated with adherence to preventive malaria chemoprophylaxis medications. Six recent studies (four US and two French) were identified that gathered data related to malaria outbreaks and prevention 
medications in military populations (Garges, 2007; Kotwal et al., 2005; LaFon, \& Scoville, 2006; Ollivier et al, 2008; Resseguier et al., 2010; Whitman et al., 2010). Four of these studies are published (Kotwal et al., 2005; Ollivier et al, 2008; Resseguier et al., 2010; Whitman et al., 2010) and two are unpublished U.S. Department of Defense Medical Command studies (Garges, 2007; LaFon, \& Scoville, 2006).

Resseguier et al. (2010) examined a limited number of individual level behavioral determinants that primarily focused on circadian rhythms in a group of French soldiers: neither KAPs nor the behavioral domains considered in this current research were included in this French study. The remaining studies primarily quantified adherence and/or addressed medical issues relative to malaria prevention and/or treatments. Each study reported poor adherence to malaria chemoprophylaxis medications. The adherence levels ranged from $7 \%$ to $55 \%$. This is alarming to hear of behaviors such as this in our military, again considering these medication should be taken $100 \%$ of the time to maintain adequate protective serum levels (Cook, \& Zumla, 2008; GlaxoSmithKline, 2010; Sanofi-aventis, 2008; Sanofi-Synthelabo, 2007; STAT RX, 2010; Strickland, 2000; West-ward Pharm, 2010). None of these studies thoroughly examined the KAPs and or the environmental domains that are considered in this study relative to this behavior.

Each of these military studies relied on self-report and participants were not always guaranteed anonymity. Reports accounting for higher adherence levels (i.e., $\geq 50 \%$ ) were suspect as overestimates due to false reports: presumably from concerns of anonymity and fears that negative repercussions may result from reporting true or lower adherence (Garges, 2007; LaFon, \& Scoville, 2006; Whitman et al., 2010). These findings emphasize the need to ensure full anonymity. Specific levels of adherence found in the limited literature relative to military populations are described and illustrated in the subsequent accounts below. . 
When Whitman et al. (2010) interviewed 44 Marines with malaria; the self reported level of adherence was $55 \%$. Serum markers taken from the same group revealed only $10 \%$ had serum levels of the medications to achieve adequate protection (Todd et al., 1997).

A larger study of 775 U.S. Army Rangers surveyed after returning from Afghanistan (Kotwal et al., 2005) found that only 52\% were adherent with their malaria chemoprophylaxis medications while in Afghanistan and only $42 \%$ had been adherent with the terminal prophylaxis after returning to the United States. The overall adherence to both weekly medications and/or terminal prophylaxis medications was only $31 \%$.

Data collected in 2006 from a non-voluntary and non-anonymous questionnaire administered to 173 Army soldiers returning from Afghanistan at Fort Lewis, WA revealed selfreported adherence levels to Doxycycline, while in Afghanistan, ranged from $18 \%$ to $62 \%$. Their adherence to Primaquine (terminal prophylaxis) after returning to the United States was only 21\% (LaFon, \& Scoville, 2006).

A similar yet anonymous self-report and self administered survey conducted at Fort Drum, NY of soldiers again returning from Afghanistan, analyzed data from 1096 completed surveys. The data revealed self-reported adherence levels to Doxycycline ranging from $7 \%$ to $55 \%$. Most of the soldiers who completed the survey (92\%, or 950 of the 1096$)$ were deployed to Afghanistan for greater than ten (10) months. Adherence levels to Primaquine (terminal prophylaxis) were not measured (Garges, 2007).

A non-voluntary, non-anonymous study of a battalion of French soldiers (300-400 soldiers) who were sampled after returning from Afghanistan revealed Plasma concentration levels of the malaria chemoprophylaxis medications they were prescribed that corresponded to levels equal to only $36.6 \%$ adherence (Ollivier et al, 2008). 


\section{A Case Study with No Cases: A Directly Observed Therapy Perspective Within a Military Population}

Despite the predominate finding literature of articles that examine inadequate levels of adherence to malaria chemoprophylaxis medications, a case study by Houston and Tuck (2005) was identified that noted very high, yet somewhat implied, level of adherence. This study was of a military population of 75-80 British personnel deployed to a highly malaria endemic region of Sierra Leone. This study is included simply to show that there are potential instances where adherence to malaria chemoprophylaxis medications could be high.

This case study reported their levels of adherence within its population of $75-80$ British military personnel in a rather uncommon manner, which is somewhat anecdotally and indicated implied the adherence levels rather than directly reporting them as a result of some sort of scientifically relevant measurement tool (e.g. survey instrument, serum drug levels, etc); therefore, I feel its analysis somewhat lacks scientific rigor.

To elaborate, this case study did not note any actual percentage levels or statistical data to support their claim of high adherence; but boasted that of the 75-80 personnel who deployed to this highly endemic region of Sierra Leone and reported no cases of malaria. The lack of any known cases of malaria does not conclude that is was due to high adherence to.

The high level of adherence in Houston \& Tuck's case was attributed to the military leadership hierarchy, commonly referred to as the "chain-of-command." The chain-of-command took an active role in ensuring that all military members adhered to the physician prescribed preventive therapies, including malaria chemoprophylaxis medications, without exception (Houston \& Tuck, 2005). These actions strongly resemble those see in the different variations of Directly Observed Therapy (DOT), where the ingestion of a medication is directly observed by a responsible individual (McPhee et al., 2007). 


\section{Other Directly Observed Therapy Findings}

The literature reports many other examples of DOT producing very high adherence levels in other medical therapies. The Centers for Disease Control and Prevention recommend DOT for all drug resistant tuberculosis patients particularly due to the high level of adherence it can produce, many of which approach $100 \%$ (McPhee et al., 2007). The DOT approach is a reasonable option in some and required in others; nevertheless, it is not a perfect remedy, particularly for military populations.

Researchers typically utilize DOT when adherence is imperative to control and to support the validity of their results (Grebely et al, 2007). Complicated treatment regimens as with methadone maintenance patients receiving concurrent Chronic Hepatitis $\mathrm{C}$ treatments show significantly improved virologic responses with DOT (Bonkovsky et al, 2008). A 2008 randomized trial, found $21 \%$ (54\% vs. $33 \%$ ) greater levels of sustained virologic response in DOT patients over self administration patients (Bonkovsky et al., 2008).

Modified versions of DOT have also shown to improve adherence. In a 2004 randomized trial to assess a modified version of DOT (m-DOT); 234 HIV infected adults located in Mombasa, Kenya receiving antiretroviral therapy were visited by a nurse twice weekly to observe ingestion of their medications (Sarna et al., 2008). Adherence in these m-DOT patients improved $480 \%(P<0.001)$, with final adherence levels reported at $82 \%$.

A 2009 community based DOT study in Lima, Peru provided community health workers to visit the homes of impoverished antiretroviral therapy (ART) patients twice daily to directly observe the ingestion of ART medications and also offer additional medical, social, and psychological support. This community-based DOT program not only improved virologic suppression and psychosocial outcomes of the ART patients, but it also reportedly extended further into the community to help reduce HIV stigma (Munoz et al, 2009). 
Similar DOT-like actions were recently taken by U.S. commanders in Haiti when they determined that U.S. military personnel were not taking their medications properly. This prompted military commanders to order greater observation by supervisors to enforce and insure adherence to these medications (Robson, 2010). The results of these actions, whether they improved adherence levels or not, are unknown at this time. No studies have been conducted and released relative to these actions in this population.

Despite its successful application in multiple settings (Bonkovsky et al., 2008; Grebely et al., 2007), strictly controlled versions of DOT or the many variants of this therapy are not the solution for military populations that may number in the thousands and deploy or operate around the world on missions requiring multiple schedule variations, with austere conditions, requiring rapid and frequent deployments, and limited opportunities to practice this means of control.

To the non-military individual, it may seem intuitive that a DOT type system of controlling adherence to medical therapies would be ideal for military populations. These populations are highly motivated, disciplined, highly responsive, and able to follow instructions, and have multiple levels of direct supervision. Though it is very possible to conduct this type therapy and it happens often, it is also very expensive and requires yet another level of oversight that is not conducive to an effective military force.

In lieu of resorting to a DOT form of control, Military commanders and Public Health organizations endeavor to better understand the behaviors and issues that affect the actions of their personnel, and prefer to seek more reasonable means of controlling behaviors in their populations (Garges, 2007; Kotwal et al., 2005; LaFon, \& Scoville, 2006; USAPHC, 2010; USARCENT, 2006; USSOUTHCOM; 2004.)

\section{Phenomena Affecting Adherence to General Medical Therapies}

The corpus of research studying adherence to malaria chemoprophylaxis medications, particularly in military populations, is limited. The literature supporting adherence to general 
medical therapies is much more complete and extensive; therefore, the phenomena known to affect general medical therapies must be examined in order to better understand and explore the potential depth, complexity, and multifaceted dimensions that may exist relative to this behavior in military populations.

The phenomena affecting general medical therapy behaviors are generally classified into and barriers, factors, and determinants (Golin et al., 2002; Hays et al., 1994; Martin et al., 2005; NCPIE, 2007; Osterberg \& Blaschke, 2005; Protopopescu et al., 2009; WHO, 2003). These terms can have similar meanings and can contain phenomena that affect adherence similarly, overlap, and/or be categorized by more than one term. For example, inadequate providerpatient communication could be categorized as either a barrier (NCPIE, 2007), a determinant (Osterberg \& Blaschke, 2005), or a factor (Bosworth et al., 2005). The major barriers, factors, and determinants found in the literature are further discussed here.

\section{Barriers}

According to the Merriam-Webster dictionary, barriers (2012) are something being either material or immaterial that may impede, block, or separate something. The definition also refers to them as factors that impede or restrict individuals. A recent report by the RAND Corporation reviewing 67 articles concerning the barriers to adherence to medical therapies listed the "five most commonly studied potential barriers..." (2009, p. 10). These are listed in descending order below:

1. Depression

2. Beliefs about Medication

3. Number of Prescriptions

4. Social Support

5. Perceived Health Status 
The barriers discussed in the subsequent paragraphs have been further classified into three categories: Patient, Provider, and System barriers.

Patient barriers can include (a) forgetfulness, (b) feeling that taking medications are not a priority, or having an insufficient understanding of the regimen, (c) low health literacy, (d) depression, low family or social support (Amico, 2009; Bosworth et al., 2005; DiMatteo, 2004; Forbes, 2010; Kalichman et al., 1999; Kravitz et al., 1993; Martin et al., 2005; Osterberg \& Blaschke, 2005).

Provider barriers can include (a) lack of knowledge, or tools to measure adherence, (b) use of complex treatment regimens, (c) inadequate provider-patient communication, (d) insufficient explanation of side effects and/or benefits, (e) insufficient compliance with treatment guidelines (Albert et al., 2009; Bosworth et al., 2005; Martinet et al., 2005; Patel et al., 2004; NCPIE, 2007; WHO, 2003, 2004).

Healthcare system barriers can include (a) high cost of medications, (b) limited access to care and selection of medications, (c) strict privacy requirements (Osterberg \& Blaschke, 2005; NCPIE, 2007; WHO, 2003)

\section{Factors}

According to the Merriam-Webster dictionary, factors (2012) are something that actively contributes to the production of a result. As the definition depicts, this could be the broadest term and may contain any number of phenomena that affect adherence. Most factors identified in the literature are linked to specific disease processes within numerous treatment regimens; however, there are many commonalities and the major factors that are noted in the literature and applied to general medical therapy adherence can be extrapolated.

A 2008 study by AstraZeneca of 200 physicians produced a list of the top-10 factors, presented by their patients, as affecting adherence to medical therapies (Koroneos, 2008). The list is displayed below in descending order from 1 to 10 , with percentage ranking scores: 
1. Affordability of medications $-20 \%$

2. Patient does not feel the medication is necessary $-20 \%$

3. Side effects of the medication - $17 \%$

4. Forgetfulness - $16 \%$

5. Patient not concerned about his/her condition (seriousness of illness) - $5 \%$

6. Lack of information concerning the disease or treatment - $5 \%$

7. Lack of information concerning the medication - $5 \%$

8. Inadequate family or social support - $5 \%$

9. Low level of satisfaction with the prescribed medication - $3 \%$

10. Poor relationship with the physician - $2 \%$

Specific factors have been identified in the general medical therapy literature which is potentially relevant to this current research. These include a past failure and/or success of treatment, individual patient characteristics, specific concerning the individual therapy, and both social support and clinical support networks (Klein et al., 2006; Protopopescu et al., 2009). The ability to recall medical advice and a healthy level of cognition have been shown to be important factors (Bosworth et al., 2005; Kravitz et al., 1993).

Another factor identified in the general medical therapy literature that may be of particular importance to military populations can include depression (Forbes, 2010; Kalichman et al., 1999; Martinet al., 2005) and stress related coping skills (DiMatteo et al., 1993; Bosworth et al.; 2005; WHO, 2003). Additionally, health literacy (Kalichman, Ramachandran, \& Catz, 1999, WHO, 2003), patient-provider communication (DiMatteo, 1993; Bosworth et al.; 2005; WHO, 2003); as well as current policies, laws, and regulations (Bosworth et al., 2005, NCPIE, 2007; WHO, 2003) could also be very relevant factors to military populations. 


\section{Determinants}

According to the Merriam-Webster dictionary, determinants (2012) are elements that identify or determine the nature of, fix, or condition an outcome of something. Determinants are predictors of adherence and their context usually implies a directional affect. For example, high social support is considered a determinant predicting higher levels of adherence (DiMatteo, 2004), while low social support predicts non-adherence (WHO, 2003). The list of determinants is long, and they may also overlap with other phenomena. Again, those discussed in this review must not be considered exhaustive.

The number of determinants can be seemingly endless and they are reported differently depending on what the author's point of view is, the particular disease process, the current healthcare type and system, and what resource is used to identify them. Multiple determinants have been identified that are reported to potentially affect adherence to long-term and general medical therapies. The most common determinants that may affect this research and military populations have been extracted to report in this review and include the following (Golin et al., 2002; Sherbourne, Hays, Ordway, DiMatteo, \& Kravitz, 1992; WHO, 2003): a history of substance abuse, high dosing frequency, high complexity of the treatment regimen, poor patient insight into the disease or treatment process, and lack of adequate provider-patient relationships (i.e., trust, access, level of communication, etc.).

A history of substance abuse, particularly current substance abuse, was found to be a major predictor of poor adherence in virtually every disease process (Golin et al., 2002; WHO, 2003). This is an interesting finding and could be perceived as significant for military populations, and it may very well be; nevertheless, substance abuse is not tolerated in the military. Individuals identified with any sort of substance abuse issues are removed from any deployment and either sent into rehabilitation or processed out of the military. Furthermore, there is absolutely zero tolerance for any type of substance abuse in the Ranger Regiment or 
the USASOC. In this instance, service members are immediately reassigned and/or rehabilitated, then reassigned. No form of substance abuse is tolerated within these organizations.

There may be some histories of substance abuse within the Rangers or the USASOC, but this is may also never be known to any Commander or Medical Provider. It may never be reported outside any current substance abuse issue or incident. In other words, this may never be known until a current substance abuse issue is identified; in which case this individual will be immediately released from the USASOC. Therefore, though this is a potentially significant issue, it will most likely never be identified within the Ranger or any other USASOC population, certainly not within this current research.

Additionally, in order to gain exempt status by the current IRBs, questions that could result in legal or administrative actions that could potentially harm participant, as these would be, were not allowed or explored within the survey instrument supporting this research.

Sherbourne et al. (1992) found that a past history of non-adherence was the strongest predictor of future non-adherence in their 2-year study of 1198 hypertension, diabetes, and heart disease patients. This may be a very important determinant to consider relative to this particular study on adherence to malaria chemoprophylaxis medications. If Rangers did not adhere to these medications in the past, they may not adhere in the future either.

\section{What are "not" considered determinants?}

Though age, especially advanced age, has been found to be associated with poor adherence in several studies (DiMatteo et al., 1992; Golin et al., 2002; Kravitz et al.et al., 1993), it is not considered a significant determinant because of the many inconsistencies found in the preponderance of other research (RAND, 2009; WHO, 2003). Other characteristics such as income, occupation, race, sex, religion, level of education (including college graduates), committed or non-committed relationships (including married), ethnicity, and rural vs. urban 
populations are all also not considered reliable determinants for adherence (Amico et al.et al., 2007; Golin et al.et al., 2002; Reif, Whetten, Ostermann, \& Raper, 2006; WHO, 2003).

Despite the multiple findings relative to these demographic issues, they were included as general demographic variables within the survey instrument. The results of the associations of these determinants will be reported in Chapters IV and V.

\section{Costs Associated With Non-adherence and Poor Adherence}

Non-adherence and poor adherence unnecessarily cost the healthcare system billions of dollars in total expenditures and consequences each year. Estimates of the total healthcare costs in the United States alone vary, and range from $\$ 100$ billion (Osterberg \& Blaschke, 2005; RAND, 2009), to $\$ 177$ billion (NCPIE, 2007), to several $\$ 100$ s of billions per year (Martin et al., 2005). Non-adherence to general medical therapies accounts for 33 to 69 percent of all medical related hospitalizations in the United States (Osterberg \& Blaschke, 2005) and is a factor in an estimated 125,000 deaths each year (Martin et al.et al., 2005).

This enormous cost burden may be preventable, and studies have demonstrated the potential savings from interventions to improve adherence and reverse this trend (Cleemput et al.et al., 2002; Nachega et al.et al., 2010; NCPIE, 2007; Rizzo, Abbott, \& Pashko, 1996). For example, Cleemput et al.et al. (2002) found that improving adherence to medical therapies could reduce absenteeism at work and save employers $\$ 290$ per hypertensive employee, and $\$ 1710$ per depressed employee (expressed in dollar values for the year 2000). Nachega et al.et al. (2010) found that improving adherence in antiretroviral therapy (ART) patients in South Africa, where medications are free, decreased the overall direct healthcare costs per patient by \$85 per month.

Optimal adherence saves money; how it is appreciated depends on the effort, time, and location. It can be summed up in a quote from Cleemput et al.et al., "The cost-effectiveness of 
compliance-enhancing interventions will depend upon the costs and health effects associated with usual care and the intervention's own costs and health effects" (2002, p. 90).

These cost related studies and findings associated with poor adherence further support the justification and appropriateness of this study within military populations. The potential monetary savings associated with the prevention of malaria infections to military members deployed to combat settings are illustrated in an ethical justification previously conducted by the Author and can be reviewed further in Appendix 1.1. In this justification, the prevention of only one malaria infection could save nearly $\$ 18,000$ in evacuation costs alone Nevertheless, when these costs are defined in human suffering or the potential loss of life, they can never be fully conceptualized or estimated.

\section{Theoretical Models Used to Support Adherence to General Medical Therapies}

Many theoretical models have the potential to explain and support studies on adherence to medical therapies at both the individual and community levels, including traditional and emerging theories. The flexible applicability of most health promotion theories, coupled with the widespread impact adherence may have on health, provides ample opportunity to utilize a variety of theoretical models (DiClemente, Crosby, \& Kegler, 2009).

A comprehensive application of all possible theoretical models and frameworks is not within the limits of this review; nevertheless, the models identified within this literature review are listed within Appendix 1.2. Three of these theories, the Health Belief Model (HBM), the Theory of Reasoned Action/Theory of Planned Behavior (TRA/TPB), and the Social Cognitive Theory (SCT) were actually chosen to support this current research. Further discussions and explanations of these three theories are included in Chapter III because they were integral in the initial to conceptualization of the study and design of the survey instrument. 


\section{Strategies of Improving Adherence to General Medical Therapies}

The review of literature on medical adherence has provided a number of different intervention strategies to improve adherence. Though this research focuses on understanding the behaviors associated with adherence to malaria chemoprophylaxis medications and how those behaviors impact adherence to these medications, this research will also inform and guide future interventions and research aimed at improving this adherence in this populations. Therefore, it is reasonable that the practices, paradigms, and programs that are considered beneficial to improving general medical therapies be briefly reviewed. These will be addressed further in Chapter $V$ concerning the significant results of this research and the potential for future research.

The strategies to improve adherence to general medical therapies vary somewhat between each literary presentation. The literature tends to group intervention strategies into broad categorical approaches and then list specific strategies to support them. The strategies within the literature also vary between specific disease processes. In any case, multiple strategies to improve adherence to general medical therapies are readily available within the literature and a general overview those strategies are presented in this review.

The National Council on Patient Information and Education (2007) identified what they consider the core strategies for improving medical adherence, and ten priorities for action. None of these strategies or priorities acts in a vacuum. To ensure success they are best applied in combination and require multidisciplinary support. These are presented in Figures 1.1 and1.2. 
Figure 1.1. NCPIE, Strategies for Improving Patient Adherence

- Recognize the disease characteristics of non-adherence

- Increase public awareness, through a national public education campaign

- Patient information strategies, to increase knowledge of treatment and disease processes

- Behavioral reinforcement and patient support, to improve and simplify treatments

- Strategies directed at health professionals to improve provider-patient relationships

- The need for a multidisciplinary approach to improve adherence

- The need for supportive government policies

- The need for research support and research rigor

Note. From "Enhancing Prescription Medicine Adherence: A National Action Plan," The NCPIE, 2007, pp. 19-24.

Figure 1.2. NCPIE, Ten Priorities for Action

- Elevate patient adherence as a critical health care issue.

- Agree on a common adherence terminology that will unite all stakeholders.

- Create a public/private partnership to mount a unified national education campaign to make patient adherence a national health priority.

- Establish a multidisciplinary approach to compliance education and management.

- Immediately implement professional training and increase the funding for professional education on patient medication adherence.

- Address the barriers to patient adherence for patients with low health literacy.

- Create the means to share information about best practices in adherence education and management.

- Develop a curriculum on medication adherence for use in medical schools and allied health care institutions.

- Seek regulatory changes to remove road-blocks for adherence assistance programs.

- Increase the federal budget and stimulate rigorous research on medication adherence.

Note. From "Enhancing Prescription Medicine Adherence: A National Action Plan," The NCPIE, 2007, pp. 25-27.

The WHO (2003) presents a philosophy of adherence that utilizes five dimensions and five major interventional categories, which they recommend be considered in any situation where improving adherence to medical therapies is the goal (see Figure 1.3). 
Figure 1.3. WHO, 5-Dimensions of Adherence

- Social and economic factors and interventions

- Health system and health care team-related factors and interventions

- Therapy related factors and interventions

- Condition related factors and interventions

- Patient related factors and Interventions

Note. From "Adherence to Long-term Therapies: Evidence for Action," The WHO, 2003, p. XI.

When presenting these dimensions, the WHO (2003) maintains that the factors affecting adherence are complex and require multidisciplinary efforts. Figure 1.4 notes the major interventional categories that should be considered. These are again best applied in combination.

Figure 1.4. WHO, Five Major Interventional Categories

- Patient education

- Behavioral skills

- Self-rewards

- Social support

- Telephone follow-up

Note. From "Adherence to Long-term Therapies: Evidence for Action," The WHO, 2003, p. XI.

Osterberg and Blaschke (2005) propose there are four major interventional categories that they recommend necessary to improve adherence to medical therapies. These categories are presented in Figure 1.5. 
Figure 1.5. Osterberg and Blaschke, Four Major Interventional Categories

- Patient education

- Educational interventions for patients and family members

- Improved dosing schedules

- The use of organized pillboxes

- Simplifying the dosing regimen as much as possible (e.g., daily dosing)

- Cues to remind patients to take their medications (e.g., cell phones and pagers)

- Increased office hours

- Including evening hours

- Create shorter wait times

- Improve delays (e.g., better parking, improved transportation)

- Enlist ancillary providers

- Improved communication ${ }^{\mathrm{a}}$ between physicians and patients

Note. Adapted from "Adherence to medication," by L. Osterberg and T. Blaschke, 2005, The New England Journal of Medicine, 353(5), p. 491.

${ }^{a}$ Key Factor to boost adherence

Cleemput et al.et al. (2002) suggest that there are five major interventional categories to improve adherence to medical therapies. These five categories are presented in Figure 1.6.

Figure 1.6. Cleemput et al.et al., Five Major Interventional Categories

\section{- Counseling \\ - Patient education \\ - Reminders \\ - Less complex treatment regimens \\ - Improved communication \\ - Other forms of increased supervision \\ Note. Adapted from "A Review of the Literature On the Economics of Noncompliance. Room for Methodological Improvement," by I. Cleemput, K. Kesteloot, and S. DeGeest, 2002, Health Policy, 59(1), p.90.}

The ideas of the different authors and organizations vary somewhat; nevertheless, a general consensus can be deduced and translated into to five major categories:

1. Improve patient, family member, and caregiver education

2. Support greater awareness of and identification of non-adherence

3. Improve dosing and total management of disease processes 
4. Improve communication and relationship between provider and patient

5. Utilization of some form of patient dosing reminder

\section{Phenomena Affecting Adherence to Malaria Chemoprophylaxis Medications in Civilian Populations}

The corpus of literature concerning behavioral phenomena affecting adherence to preventive malaria chemoprophylaxis medications in the civilian populations is relatively limited, but represents a far greater amount of research than that relevant to military populations. Despite limited research, the civilian population studies do identify similar phenomena as those found in the general medical therapy literature. For example, two studies found that limited travel education (Hymel and Yang, 2008) and low personal risk perceptions (Ben-Ami et al, 2005) may be associated with poor adherence to these medications. Adverse events or unwanted side effects have also been recognized as issues that contribute to poor adherence (Kitchener, Nasveld, Gregory, \& Edstein, 2005). Additionally, as with the general medical therapies, researchers recommend that a greater emphasis be placed on physician guided education to improve cognition and awareness of the need to take these preventive medications as directed (Baudon \& Martet, 1997; Ben-Ami et al., 2005; Berg, \& Visser, 2007; DupouyCamet, Yera, \& Tourte-Schafer, 2003).

\section{Phenomena Affecting Adherence to Malaria Chemoprophylaxis Medications in Military Populations}

As previously noted, the corpus of literature concerning phenomena affecting malaria chemoprophylaxis adherence in military populations are very limited. In fact and as previously noted, only one military study of French soldiers was identified that focused understanding determinant behaviors in any detail (Resseguier et al., 2010). This French study focused only on circadian rhythm behaviors, unrelated to the KAPs and behavioral domains explored by this current research. The remaining studies simply quantified basic reasons given for non- 
adherence and again did not fully explore the knowledge, attitudes, and practices relevant to this behavior.

Despite this limitation, those limited phenomena that have been identified within these military populations again appear to follow the general medical therapy literature. For example, two unpublished military studies examining malaria outbreaks in U.S. military populations identified similar phenomena as those found in civilian malaria studies and in the general medical therapies. These two studies identified forgetfulness, side effects to medications, inadequate supply of medication, lack of pre-travel health education, low perceived threat, and marginalizing the importance of prevention as factors that impacted the study participant's ability and/or decision to follow properly adhere to malaria preventive guidance (Garges, 2007; LaFon, \& Scoville, 2006). Nevertheless, no significant associations or conclusions were drawn relative to any behaviors. These factors were simply identified and listed as findings of their nonanonymous survey questionnaire.

Given the limitations presented by the dearth of information concerning KAPs and the environmental considerations that are included in this study and thought to be associated with adherence to malaria chemoprophylaxis medications in the 75th Ranger Regiment; the phenomena known to affect adherence to malaria chemoprophylaxis medications in other military populations, in civilian populations, as well as those phenomena noted throughout the general medical therapies have been generalized to the Ranger population. These have all been considered fundamental to the conceptualization of this study.

\section{Other Military Health Behaviors: Weapon Safety and Condom Use}

The literature was void of any studies that supported adherence levels or any relevant behaviors that may explain adherence weapon safety. No studies or information was identified for any population, military or civilian. Therefore, an analogy was created in the form accidental 
discharges and weapon related injuries that were reported in the Army by the United States Army Combat Readiness and Safety Center.

Aggregate Army data maintained by the United States Army Combat Readiness and Safety Center reported very few accidents that could be attributed to the lack of weapon safety. In fact, the total number of weapon safety related accidents reported between the years of 2005 and 2010 ranged from a high of 71 in 2007, to a low of 26 in 2010 (US Army, 2010). Given the number of Army personnel, which were reported by this same organization in 2009 to be over 500,000 members, even if the high number of injuries (71 in 2007) were placed as numerator in any equation of $4-500,000$ as a denominator, this would result in a number of weapon safety accidents far less than $0.1 \%$. Therefore, given the lack of any other information or available data, the author investigator conservatively implied an analogical figure to weapon safety adherence to be greater than $99 \%$.

Condom use within military populations has been well documented in the Department of Defense survey of health related behaviors among active duty military personnel (Dept. of Defense, 2008), which is conducted every three years since 1980 . The most recent survey available is the 2008 version: the 2011 version is not yet available. The results of the 2008 version were utilized during the conceptualization of this study. Regarding condom use in those sexually active, unmarried military personnel, the 2008 survey participants that $43.6 \%$ used a condom during their last encounter.

Because of its analogical adherence level of greater than $99 \%$, it was postulated that if the key predictor variables associated with adherence to weapon safety guidance were identified, they may be helpful in guiding future interventions aimed at improving adherence to the lesser adhered to behaviors, particularly adherence to malaria chemoprophylaxis medications. This is further discussed in Chapter IV and V. 


\section{Why Knowledge, Attitudes, and Practices?}

The potential impact of military operations on malaria preventive measures, including an array of environmental considerations, as well as the obvious need to improve adherence to malaria chemoprophylaxis medications in military populations, emphasizes the need for a better understanding of this behavior. The literature supporting KAP frameworks (Al-Adhroey et al., 2010; He et al., 2010; Keating, Eisele, Bennett, Johnson, \& Macintyre, 2008; Kheir et al., 2010; Panagakou et al., 2009; Paulander et al., 2009; WHO, 2008a) appears to be an ideal consideration for this endeavor because of the usefulness these frameworks have shown in:

1. Gaining a greater understanding of phenomena affecting behaviors.

2. Informing and directing future interventions.

3. Indentifying behavior related information that may be useful for post intervention evaluations.

Each of these categories represented another dearth of information in the Ranger population and utilizing the KAP framework to construct the survey instrument proved to be an effective method. This will be further outlined in the methods and results chapters.

\section{Summary}

The review of the literature relevant to this research is vast, though some subjects alone represent a dearth of information. To capture an effective base of knowledge, this review has synthesized information on many subjects from an extensive range of sources. Pertinent aspects relative to the subjects of malaria, preventive measures, adherence to multiple medical therapies, theoretical support, and KAP frameworks were examined.

Maintaining adequate adherence to medical therapies is a rather complex and multifaceted topic. There is no single perfect answer, method, theory, or paradigm that this review has identified which precisely sums up this complex behavior; hence the appropriateness of the term multifaceted. 
This review has shown that a) accurate measurements and a gold standard for adherence are required to accurately quantify levels, b) that adherence levels are low in multiple medical therapies, and c) that there are multiple phenomena that must be considered to truly capture and understand this behavior. The costs of non-adherence are great. Those associated with non-adherence to malaria chemoprophylaxis medication can potentially be as well. When these costs are defined in human suffering they can never be fully conceptualized.

Several methods and approaches have been shown to improve adherence to different medical therapies, within specific study populations. The more successful approaches appear to be those that utilize multiple methods, at multiple levels, with a multidisciplinary team. It is important to identify poor adherence and reinforce beneficial practices at both the patient and provider levels.

The literature has identified a distinct gap in the current knowledge relative to the behaviors that may be associated with adherence to malaria chemoprophylaxis medications in military populations. No studies were identified in this review that thoroughly examined the association of knowledge, attitudes, and behaviors with adherence to these medications.

The majority of the findings in this review were retrieved from scholarly articles that studied civilian populations. Due to the dynamics and environmental considerations associated with military operations and military populations; therefore, significance of these civilian research findings may vary somewhat from those that may be relative to military populations. Nevertheless, the relative dearth of studies on military populations requires that these civilian studies be generalized to military populations to inform this research.

The literature clearly supports that malaria is a health threat to those travelers who venture into malaria endemic regions of the world, including the U.S. Army Rangers. It is also clear that malaria chemoprophylaxis medications can prevent malaria. The problem is that these medications have to be taken as directed to be effective, and the impact of military can add yet 
another level of complexity that can further detract from the level of adherence that is required of the medications. A better understanding or the behaviors associated with adherence in this population, to these medications, may help to improve this equation.

In summation, there are multiple cases of non-adherence to malaria chemoprophylaxis medications in both civilian and military populations. The issues and reasons relative to this adherence are not well known, particularly in military populations.

Given the limitations presented by the dearth of information concerning KAPs and other environmental considerations associated with the behaviors that may affect adherence to malaria chemoprophylaxis medications in U.S. military populations, this literature review has captured an abundance of knowledge which has was served well to support this exploratory study. Hopefully, this will culminate in improving the health of the nation's soldiers, sailors, and airmen around the world.

\section{Critique of the Literature}

The definition of adherence that is generally accepted across the medical and public health communities has been established within this review. Nevertheless, several military authors continue to use the word compliance in lieu of the word adherence. The term adherence is the most reasonable term, given the definition given by the WHO in 2003 and the agreement between the provider and the patient as it pertains to participation in any treatment. Therefore, the use of the term adherence should not vary between populations; particularly within populations of military personnel taking malaria chemoprophylaxis medications, who should be given the respect to fully participation in their treatment options.

In the case of Houston and Tuck (2005), where it was reported that there were no cases of malaria in a population of 75-80 British military personnel deployed to Sierra Leone, it was implied that there was a high level of adherence to malaria chemoprophylaxis medications. This had the potential to not be completely accurate and must be further explored. Though there was 
an increased vigilance of the chain-of-command to improve adherence to these medication, there could have been other explanations. For example, this increased vigilance could have also increased the use of mosquito nets, protective clothing, and/or mosquito repellants; which may have also, or on their own, prevented malaria infections.

\section{Limitations}

The references that were included in this review were extracted by a single researcher, the Author, Major Michael Pagel. The majority of data was obtained through the FIU Libraries medical and public health aggregator database search engines. Multiple other sources were also utilized, including an array of private search engines, as well as government and military websites. Though this search was extensive and utilized multiple resources, there are most likely many other untapped resources. 


\section{CHAPTER III}

\section{METHODS}

\section{Statement of the Problem}

The U.S. military reported 423 cases of malaria between 2003 and 2005 (Ciminera \& Brundage, 2007; Kotwal et al., 2005). There were at least 12 cases in Haiti during the first two months of the 2010 earthquake recovery efforts, and new cases of malaria continue to be documented in U.S. military medical and public health reports (Robson, 2010; USAPHC, 2010; Whitman et al., 2010). Each U.S. military case of malaria might have been avoided had they properly taken their malaria chemoprophylaxis medications (Garges, 2007; Kotwal et al., 2005; LaFon, \& Scoville, 2006; Robson, 2010; Whitman et al, 2010).

The former Surgeon General, C. Everett Koop, is commonly quoted as saying, "Drugs don't work if patients don't take them," which is especially true with malaria chemoprophylaxis medications (Abraham et al., 1999; Ben-Ami et al., 2005; Dupouy-Camet et al., 2003). Due to their narrow therapeutic range and relative half-life, these preventive chemoprophylaxis medications require $100 \%$ adherence to maintain efficacious levels (Cook, \& Zumla, 2008; GlaxoSmithKline, 2010; Sanofi-aventis, 2008; Sanofi-Synthelabo, 2007; STAT RX, 2010; Strickland, 2000; West-ward Pharm, 2010). Despite this requirement to maintain $100 \%$ adherence, these medications are notoriously very poorly adhered to and the reasons are not well understood, especially in military populations.

The many factors, barriers, and predictors affecting general medical adherence are often complex and vast (Albert et al., 2009; Amico et al., 2007; Bosworth et al., 2005; Cleemput et al., 2002; DiMatteo et al., 1992; DiMatteo, 2004; WHO, 2003). Adherence becomes even more complex during military training and combat operations that may detract from any medical therapy, including the strict adherence required of malaria chemoprophylaxis medications (Ciminera \& Brundage, 2007; Garges, 2007; Kotwal et al., 2005; LaFon, \& Scoville, 2006; 
Resseguier et al., 2010; Whitman et al, 2010). Therefore, it is important to better understand how to improve this behavior in military populations.

The USASOC is charged with training some of this nation's most elite fighting forces, one of these is the U.S. Army Ranger: referred to from this point on as Rangers. Rangers are known to have contracted malaria because they did not follow preventive measures as recommended, specifically by not taking their preventive malaria chemoprophylaxis medications as directed (Kotwal et al., 2005; USPHC, 2010).

Rangers operate in relative secrecy. Their deployment and training cycles take them to some of the most austere and dangerous regions of the world, many of which are endemic with malaria (USASOC, 2011a, 2011b; WHO, 2010). These active training and deployment cycles, combined with their relative secrecy, have resulted in very limited access to these populations for the purpose of any type of scientific observation.

The lack of access to this Ranger population has created a general dearth of information on a variety of topics; particularly health behaviors and the KAPs associated with them. This study will help to improve this knowledge gap.

The USASOC understands that malaria prevention measures are important and recognizes the relevant KAPs are understudied, particularly in their Ranger population assigned to the 75th Ranger Regiment.

In an effort to improve public health support and improve future health outcomes in the Ranger population, the USASOC and the 75th Ranger Regiment Commander have granted access to the Ranger population for this KAP study and therefore, the Rangers of the 75th Ranger regiment are the study population of this research.

\section{Purpose of the Study}

Adherences to malaria chemoprophylaxis medications in U.S. military populations, including the Ranger population, are notoriously low. The reasons these personnel choose not 
to take these preventive medications properly are not clear. No studies exist that thoroughly examine the relevant KAPs associated with this behavior in the Ranger population. This exploratory study will fill this gap in the current knowledge and also inform future interventions aimed at improving adherence to malaria chemoprophylaxis medication in the Ranger population, as well as throughout the military. The knowledge gained from this study will be used to plan future research and interventions with the U.S. Army Public Health Command.

\section{Research Questions}

This study focused on answering three research questions:

1. What relationship, if any, exists between the current KAP domains, environmental domains, and behavioral outcomes?

2. Can any of the behavioral domain variables predict behavioral intentions, as a proxy for behavior change? Behavioral intention is the best predictor of behavioral change (DiClemente, Crosby, \& Kegler, 2009; Edberg, 2007).

3. Do variables within the environmental domains of behavioral education, behavior related training, behavior related media, or military command support moderate the relationship between any KAP domain variables? Personal characteristics are included in all the KAP domain variables.

\section{Null Hypotheses}

The three research questions were subsequently transformed into the following null hypotheses: $H_{1}$ o: No relationship exists between the current KAP domains, environmental domains, and behavioral outcomes.

$H 2_{0}$ : The current KAP domain variables cannot predict behavioral intentions, as a proxy for behavior change. 
$H 3_{O}$ : Command Support domain variables do not moderate the relationship between variables within the KAP domains of perceived susceptibility and risk, behavior related practices, interpersonal communications, and the behavior outcomes.

\section{Overview of the Study}

This study aimed to identify and understand the KAPs associated with three different health behaviors, each with varying levels of adherence (high, medium, and low). These behaviors are 1) adherence to weapon safety guidance, 2) adherence to condom use guidance, and 3) adherence to malaria chemoprophylaxis medications.

The conceptual idea of this study was that if the KAPs affecting the more highly adhered to behaviors are identified and better understood, they could be compared to those KAPs affecting the lesser adhered behaviors. This would, in concept, lead to a clearer understanding of the dynamics affecting these behaviors in this population. Once the KAPs associated with each of these behaviors was captured and better understood, this would help to improve the conceptualization, development, operationalization, and implementation of future research and/or interventions within this population. The primary focus of this study is to improve adherence levels to malaria chemoprophylaxis medications.

The dearth of data related to the three health behaviors chosen for this study, in any military population, required generalizing aggregate data from other populations to this population of Rangers. Aggregate data revealed:

1. Following Weapon Safety guidance as directed is analogously a highly adhered behavior, with levels that can be compared to 99\% or greater (US Army, 2010).

2. Condom Use in U.S. Army populations can be considered a medium adhered to behavior, with reported adherence levels of $43 \%$ (Dept. of Defense, 2008).

3. Taking Malaria Chemoprophylaxis medications as directed can be considered a low adhered to behavior, with reported levels ranging from as low as $7 \%$ to highs 
of 55\% (Garges, 2007; Hagan, Marcos, \& Steinberg, 2010; Kotwal et al., 2005;

LaFon, \& Scoville, 2006; Whitman et al, 2010).

The conceptualization of this exploratory study was based on a previous study conducted by the author that included two qualitative interviews and one focus group. The study was implemented as a cross sectional survey, and simultaneously measured demographics, KAPs, and environmental variables associated with these three behaviors. The survey instrument was a proctored, anonymous questionnaire administered to a convenience sample of volunteers at each of the five Ranger units located on three military bases in Georgia and in Washington State.

\section{Theoretical Support}

Theoretical support for this study is based on components of three health behavior theories; the Health Belief Model (HBM), the Theory of Reasoned Action/Theory of Planned Behavior (TRA/TPB), and the Social Cognitive Theory (SCT). Together, these theories can explain the complex behavioral phenomena associated with adherence to malaria chemoprophylaxis medications through the fundamental concepts of (a) perceived severity, benefits, barriers, and susceptibility; (b) behavioral intentions, attitudes towards behavioral outcomes, power, and control; and (c) self efficacy, vicarious learning, reinforcement, reciprocal determinism; and the interaction between the individual, the environment, and the behavior. A model is also provided below to give a visual representation of how these concepts pertain to this study.

The components of the HBM can be easily adapted to the health behaviors in this study (DiClemente, Crosby, \& Kegler, 2009; Edberg, 2007). The HBM has six components: perceived susceptibility, perceived severity, perceived benefits, perceived barriers, cues to action, and self-efficacy. The first four components are of particular interest to this study (perceived susceptibility, perceived severity, perceived benefits, and perceived barriers). These 
components also have close relationships to the themes indentified in the previous interviews and focus group conducted by the author, which will be discussed in Chapter III.

To understand how the TRA/TPB applies to this study, it is best to first divide it into its respective parts, the TRA and TPB. The TRA considers behavioral intentions, attitudes, and subjective norms as predictors of behavior (DiClemente, Crosby, \& Kegler, 2009; Edberg, 2007). According to the TRA, behavioral intentions are the likelihood to perform the behavior and are the best predictors of actual behavior. Behavioral intentions are a functional result of both attitudes and subjective norms. Attitudes include those directed toward a behavior, or more precisely, attitudes toward the expected outcome of a behavior. Subjective norms are the influence other people have on a person's attitudes and behavior. In the case of this study, these norms are related to whether the behavior is perceived as approved or disapproved.

According to the TPB, perceived behavioral control and perceived power are predictors of behavior (DiClemente, Crosby, \& Kegler, 2009; Edberg, 2007). Perceived control is a belief towards factors that will make it easy or difficult to perform the behavior. Perceived power is conceived as the power one has to perform the behavior.

Combined, the TRA/TPB (sometimes referred to only as the TPB) is well suited to be applied to behaviors that are under a person's volitional control. Each of the three behaviors examined in this study are under the volitional control of a subject.

Behavioral intentions, attitudes, and subjective norms supported by the TRA/TPB are particularly applicable and were captured in the survey instrument. Subjective norms were represented by descriptive and injunctive norms in the questionnaire. Injunctive norms are those behaviors of that are perceived as being approved by others. Descriptive norms are perceptions of one's approval of how others are behaving. 
Finally, the TRA/TPB is known to be useful at identifying how and where to target strategies for changing behaviors. This is important because this exploratory study will inform future interventions throughout the military.

The Social Cognitive Theory (SCT) is perhaps the most appropriate theory to this study because of its ability to explain the interaction of individuals within their immediate environment and the principal of operant conditioning where individuals learn behaviors through positive and negative reinforcements (DiClemente, Crosby, \& Kegler, 2009; Edberg, 2007). Reinforcement is addressed throughout the survey instrument and command support has been hypothesized as a potential moderator in this study.

The components of the SCT are divided into six individual characteristics and four environmental factors. The six individual characteristics include: self efficacy, behavioral capacity (knowledge and skill), expectations (outcomes), expectancies (good outcomes or rewards), self control (control over change), and emotional coping (ability to deal with change). The four environmental factors include: vicarious learning (through observing consequences), situation (perception of the environment), reinforcement (positive or negative responses to behavior), and reciprocal determinism (individual factors/social cues==>receive response==>adjust behavior, and so on). Each of these concepts is measured in the survey instrument and is relevant to the themes identified in the previous qualitative interviews and focus group conducted by the author.

\section{Models}

A model was created that depicts the theoretical framework supporting this study. The model depicts both the change effects of the mediators, and the confounding interactions of the moderators on the independent variables and behavior outcomes (see Figure 3.1). 
Figure 3.1. Theoretical Diagram Model: Mediator Effect and Moderator Interaction

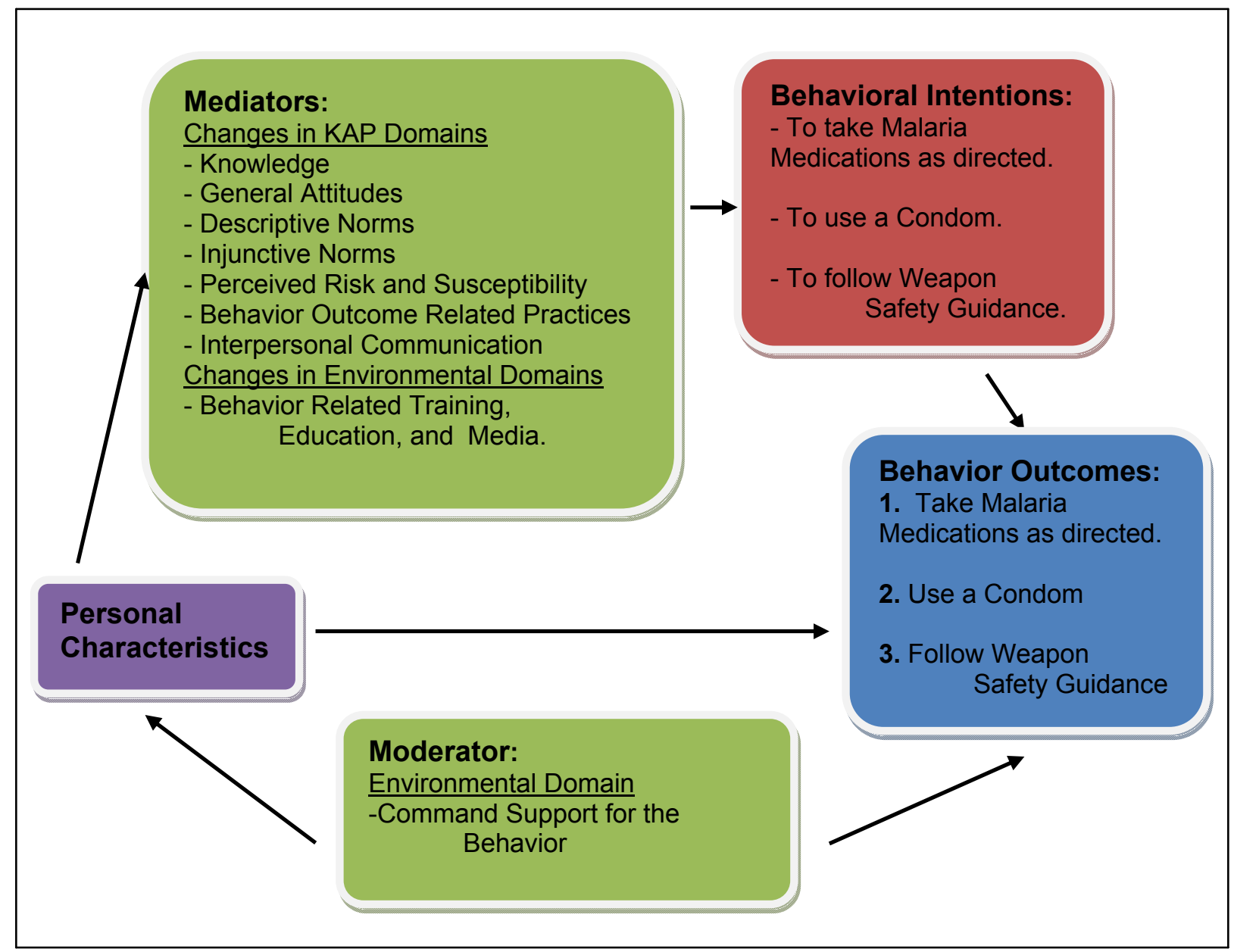

\section{The Study Framework}

Given this study's exploratory nature, the dearth of information relative to malaria prevention behaviors in the Ranger population, the KAP framework was considered ideal for identifying and understanding phenomena associated with each behavior. The KAP framework is well utilized in the literature, particularly when the findings are anticipated to aid future interventions, which is an over arching future goal of this study (Al-Adhroey et al., 2010; He et al., 2010; Keating, Eisele, Bennett, Johnson, \& Macintyre, 2008; Kheir et al., 2010; Panagakou et al., 2009; Paulander et al., 2009; WHO, 2008a). 


\section{Previous Qualitative Interviews and Focus Group}

As noted and discussed above; the domain variables within the survey instrument were not only identified from literature review, based on theoretical support of three health behavior theories, but also each variable is closely linked to the two interviews and one focus group the author conducted during the fall semester of 2009 , as part of his graduate studies requirements in PHC 7493, Qualitative Studies. The full report of this research is available upon request.

This was a small and independent study of nine anonymous Special Operations Forces volunteers. It included two semi structured interviews and one focus group of seven. Each session was recorded on digital media and then transcribed verbatim into individual word documents. These were then coded and analyzed through Atlas.ti@ qualitative data software. This analysis identified multiple themes that were considered to affect adherence to malaria chemoprophylaxis medications within this group of volunteer.

The information from this analysis has significantly contributed to the formulation of the hypotheses presented in this dissertation, as well as the fundamental conceptualization and design of the survey questionnaire.

The analysis identified four (4) categories of 21 themes that were thought to affect adherence to malaria chemoprophylaxis medications in this group. Key concepts included elements of knowledge, perceived risk and susceptibility, education, communication, and reinforcement. The four categories are as follows:

- Four themes were identified that may greatly influence inadequate adherence to malaria chemoprophylaxis, in this sample.

1. The perception that malaria will never infect them. It can happen to others, but not them.

2. The perception that they know the malaria threat is low or non-existent in their area better than any scientific or medical advice. 
3. The perception that malaria is not a threat because they have not personally seen any cases of malaria.

4. The perception that taking malaria medications is not important compared to high priority tasks, especially in a combat environment.

- Eight themes were identified that were considered a lesser influence and may somewhat influence inadequate adherence within this group.

1. No immediate level planning was considered for the threat of malaria.

2. Personnel forget to take their medications as directed and skip doses.

3. Side effects and reactions to medications interrupt dosing.

4. The perception that there are no enforceable repercussions to compel the individual to take medications involuntarily.

5. The perception that malaria is not a serious disease and it is not a true threat to their health.

6. Malaria is not a daily or even periodic consideration in one's mind.

7. Some individuals do not like taking any medications, especially pills, and particularly when they are not currently ill.

8. Personnel do not have the medications nearby at regular dosing intervals.

- $\quad$ Three themes were identified that may greatly influence adherence.

1. The perception that the malaria threat is real and near.

2. The perception that if others know of your inadequate adherence, your actions may influence them to do the same.

3. The perceptions that your character may be compromised if others know you are intentionally not adhering to your medication regimen.

- Four themes were identified that may somewhat influence adherence.

1. Personnel would take their medications if reminded regularly. 
2. When the perceived threat of malaria is high, side effects will not stop personnel from taking their medications.

3. The perception there will be real financial repercussions if adherence to medications is not maintained.

4. Frequent discussions or information reminding personnel of the dangers and threat of malaria.

The results of the final qualitative analysis were then synthesized into five key findings:

1. Malaria must be perceived as a serious disease and personal health threat.

2. Medical and preventive advice must be perceived as accurate.

3. Chemoprophylaxis medication use must be monitored in some form.

4. Travelers must continually be aware of the malaria threat.

5. Malaria and malaria chemoprophylaxis medications must be included in the mission planning process.

\section{Instruments and Measures}

A questionnaire was constructed utilizing the KAP framework as described above. The format was adapted from a behavioral KAP questionnaire previously utilized by U.S researchers (Anastario, 2010) observing norms, risky sexual behaviors, and HIV stigma in Barbados military members. The questionnaire content is a creative synthesis of themes indentified from the previous interviews and focus group, the literature review presented in Chapter I, and a small amount input from my 23 years of personal observations as a special operations soldier and health care provider. The final version of this questionnaire is available in Appendix 3.1, titled the USASOC Health Behavior KAP Questionnaire. The codebook is an annotated version of the questionnaire and will be made available upon request from the author at cmbtdvr@hotmail.com. 
The questionnaire includes concepts form the chosen theoretical frameworks, and was constructed utilizing the basic principles of questionnaire design (Bradburn, \& Sudman, 1979; Converse, \& Presser, 1986; Payne, 1951; Sudman, \& Bradburn, 1983). The result is a virtually new questionnaire. New variables were created, and additional environmental domains were included to capture phenomena unique and important to U.S. military populations. These environmental domains include (a) health behavior related education, (b) health behavior related training, (c) health behavior related media, and (d) command support for the behavior.

\section{Pre-Construction of the Instrument}

In order to produce a survey instrument that would measure the appropriate objectives and withstand both scientific and theoretical scrutiny, a thorough understanding of survey questionnaire design was considered of principal necessity. To sufficiently justify the rigor that

went into creating a complex questionnaire, one that simultaneously measures three behaviors, a firm grasp of the fundamentals of questionnaire design was necessary. The details of the complex learning experience are outlined in bullet format in a paper created by the author (Pagel, 2010) and are presented in Appendix 3.2.

The construction of the survey instrument was drawn from four sources:

1. Asking Questions: A Practical Guide to Questionnaire Design (Seymour, Sudman, \& Bradburn (1983).

2. Survey Questions: Handcrafting the Standardized Questionnaire (Converse, \& Presser, 1986).

3. The Art of Asking Questions (Payne, 1951).

4. Improving Interview Method and Questionnaire Design (Bradburn, \& Sudman, 1979). 


\section{The Final Survey Instrument}

The final survey instrument utilized the aforementioned KAP framework. It was presented in a 65-page (two sided) hard copy spiral bound booklet format and contained 341 questions. It was administered anonymously. No identifying information that could directly link any participant to their responses was included on the questionnaire. The time for completion was approximately 90 minutes. Some study participants finished the questionnaire in as little as 30-40 minutes, while only a few took more than 90 minutes. The questionnaire is very thorough, contains detailed instructions, and is divided into five sections:

1. Demographic Variables, containing 17 questions

2. Malaria Chemoprophylaxis Variables, containing 108 questions

3. Condom Use Variables, containing 109 questions

4. Weapon Safety Variables, containing 104 questions

5. Administrative Questions, which comprised only 3 questions

The variables within the Malaria Chemoprophylaxis, Condom Use, and Weapon Safety sections were organized in the following two categories of eleven domains:

KAP Domains:

1. Behavior Related Knowledge

2. General Behavioral Attitudes

3. Descriptive Norms

4. Injunctive Norms

5. Perceived Susceptibility and Risk

6. Behavior Outcome Related Practices

7. Interpersonal Communications

Environmental Domains:

8. Behavior Related Education 


\section{Behavior Related Training}

\section{Behavior Related Media}

\section{Command Support}

The base questions of the questionnaire are those related to malaria chemoprophylaxis medications, which is also the primary subject of the study. To allow for reasonable comparisons, the questions related to condom use and weapon safety were subsequently created based on the content and context of the malaria chemoprophylaxis medication questions. Each of the themes identified in the interviews and focus group conducted in the fall of 2009 was closely linked to the majority of the questionnaire variables, across each of the three behaviors. In fact, there are very few variables that are not directly linked to these themes.

The questions within the survey instrument parallel each other very closely across the three behaviors, with each question being asked as closely as possible, across the three health behaviors. The response possibilities are also exactly the same across the three behaviors (e.g., dichotomous, 3-scale, 4-scale, etc.). There are few exceptions to this: most of which are in the knowledge category of each behavior, which due to the subject matter differences (i.e. malaria, condoms, and weapons) are somewhat different across the three behaviors, yet with equal skill levels and difficulty.

There are no military documents or task lists that dictate the level of knowledge a military member should have relative to either malaria chemoprophylaxis medications or condom use. Therefore, the knowledge questions for condoms and malaria were developed based on the author's previous observations and understanding of the basic knowledge military healthcare providers (HCP) consider appropriate for their population of patients. The author is currently an actively practicing military HCP and has been credentialed as Physician Assistant for the past 14 years. 
Despite the lack of any formal document or literature that state the level of knowledge military member should possess relative to malaria chemoprophylaxis medications and/or condom use, there are documents that dictate the level of knowledge a military member should have relative to weapon safety. The document that was referred to in this case is the US Army Skill Level-1 Task List (USA HQ, 2009). This task list document identifies and outlines the proper use of military weapons. All Rangers follow this task list; therefore the questions within the survey instrument were formulated and synthesized directly from this task list. No facts or U.S. Army guidance were altered in any form.

The questionnaire was edited and revised several times between November 2010 and August 2011, when the final version 1.2 was submitted for print. These edits and revisions were accomplished with the assistance of a panel of experts; nevertheless, I was the initial author and both participated in every edit and I was the final approval source of all revisions.

The most important panel of experts who participated in the production of the questionnaire, were personnel at the Walter Reed Army Institute of Research (WRAIR), Military HIV Research Program's (MHRP) Data Coordinating and Analysis Center (DCAC). The Data Analysis Specialist who had the highest level of input and participation in the editing and revisions process of this questionnaire was Mr. Greg M. Pfeiffer. Mr. Pfeiffer and I had daily communications during this process and the final product was produced with the utmost quality. Responses were first planned to be presented in a true Scantron@ format, but the Scantron@ licensing was determined to be cost prohibitive for this survey; therefore, responses were given in the booklet in a Scantron@-like format that required manual entry into the database.

A detailed description of Mr. Pfeiffer's and the other DCAC professional's level of participation and duties can be found in the detailed Data Management Plan (DMP) that is located in Appendix 3.3, which includes the detailed process for creation of the ClinPlus data base that supported this research. 
This survey instrument was not pilot tested prior to administration. This was justified by two issues:

1. Due to the high operation tempo of the Ranger units, formal validation was not a practical option.

2. The exploratory nature of this study further justified administering the questionnaire as an unvalidated instrument.

The first survey was completed on September 7th, 2011 at Fort Benning, GA; almost 1year after construction began. This research will serve a one of the steps to formally validate the instrument so that it may be used an adaptation tool for future survey instruments, or possibly as the primary instrument used in future research.

\section{Study Population}

Rangers are one of our nation's most elite fighting forces, (USASOC, 2010a, 2010b). They are specially selected, very well trained, and incredibly physically fit. They are also very motivated, highly responsive, and able to follow directions well beyond that expected of most soldiers. They operate in relative secrecy, with deployment and training cycles that regularly take them to some of the most austere and dangerous regions of the world, many of which are endemic with malaria.

Following are unofficial, yet verified as reasonable semblances of the 75th Ranger Regimental general population demographics. An effort was made to request official documentation that reported the actual population demographics, as listed below, but the Regimental Headquarters could not supply this information. The reasons given were 1) the regimental headquarters does not readily have demographic data of this detail, and 2) in the best interest of Operational Security it is preferred that this data be merely verified as a reasonable semblance rather than be stated officially as the unit exact demographics. 
The total Ranger study population is approximately $+/-3500$ personnel and is an "all male" organization. The actual population varies because of gains and losses to the unit assigned personnel. Individuals may leave the unit for a number of reasons. Most often they leave either from transfers to and from other units, but may also be due an end of active service.

The Ranger units operate in 3-phases (Train, Deploy, and Rest). Due to this operational cycle, only $30-60 \%(1050-2100)$ of the total population was available to sample at any given moment during the survey.

\section{The 75th Ranger Regiment Units}

The 75th Ranger Regiment consists of five units. Each is indentified and described below:

1. Regimental Headquarters, located at Fort Benning, GA.

The Regimental Headquarters, also known as RHQ (USASOC, 2012a), is a command and control unit consisting of approximately 200 , all male personnel. This unit is overall responsible for all operations within the entire Regiment. It is entirely made up of the Commander, his special staff, and those who support the staff members. Generally, the personnel assigned to the Regimental Headquarters, due to their command orientation, consist of personnel with a higher concentration of senior ranks and those with the most experience working within the other units of the Regiment. With few exceptions, the personnel assigned this unit have had one or multiple assignments to the other Ranger units. Each person assigned to the RHQ is very familiar with the mission, and capabilities of the other units.

2. Regimental Special Troops Battalion also located at Fort Benning, GA.

The Special Troops Battalion, also known as the RSTB (USASOC, 2012a), consist of approximately 350, all male personnel from multiple ranks, levels of experience, and Military Occupational Skills (MOS). The personnel 
assigned to the RSTB are assigned to support both the Regimental Headquarters, and the three Ranger Battalions (1st, 2nd, and 3rd) on a very intimate and regular basis. With few exceptions, the personnel assigned the RSTB have had one or multiple assignments to the other Ranger units. Each person assigned to the RSTB is very familiar with the mission, and capabilities of the other units.

In general, the RSTB personnel are trained in Command and Control, Communications, Computers, Intelligence, Surveillance, and Reconnaissance. They perform many functions in support of the entire 75th Ranger Regiment and other Special Operations units that may share the same interests.

The RSTB is also in charge of providing qualified, trained and ready Rangers who will staff the many positions throughout the Regiment. The RSTB performs this mission by operating the many special training and selection courses that support the entire Regiment. These include:

a. The Ranger Assessment and Selection Program (RASP 1 and 2).

b. The Small Unit Ranger Tactics (SURT) training, which prepares Ranger candidates to attend and pass the U.S. Army Ranger School.

c. The Pre-Special Operations Combat Medical (PSOCM) course, which prepares qualified candidates for the gulling period of instruction, both practical and didactic, in advanced paramedic skills taught at the Special Operations Combat Medic course.

3. Three Major Ranger Battalions (1st, 2nd, and 3rd).

The three major Ranger Battalions (1st at Fort Lewis, WA; 2nd at Hunter Army Airfield, GA; and 3rd at Fort Benning, GA) were created after the U.S. Army 
recognized the need for elite, highly trained, and mobile reaction forces

(USASOC, 2012a). These three battalions comprise the bulk of the 75th Ranger Regiment forces. There are approximately 800 to 1000 , all male personnel assigned to each of these battalions. The majority of the personnel possess Combat Arms military occupational skills, with the greater majority being Infantry. The majority of the population within these battalions are younger and of lower rank status. With few exceptions, this is where nearly all Rangers begin their careers in the 75th Ranger Regiment.

These personnel serve as a quick reaction strike forces around the world. Each battalion has the potential to deploy anywhere in the world on very short notice. They operate in three phases (Train, Deploy, and Rest). One unit is always ready to deploy as the others train and rest. When deploying, the major battalions can draw from the Regimental Headquarters or the Regimental Special Troops Battalion as augmentation; therefore, these battalion populations are also often a mixture of personnel from the different units, most often the RHQ or RSTB. Nevertheless and again with few exceptions, the personnel within these battalions have had one or multiple assignments to the other Ranger Battalions, the RHQ, or the RSTB. Nevertheless, even the younger and less experienced personnel within these units are very familiar with the mission, and capabilities of all the units within the 75th Ranger Regiment.

\section{Similarities within the Ranger Population}

Though the Regimental Headquarters and the Special Troops Battalion contain somewhat different variations of demographics including a smaller population, more senior personnel, a generally higher rank structure, and a more diverse range of military occupational skills (MOS); the populations remain relatively similar in nature considering their core military values, standards, and ethos. 
Due to the non-exceptional policies that govern assignment to the 75th Ranger Regiment; the Ranger populations are known to be very a homogenous group in relation to the similarities that exist across the different Ranger units relative to the core military values, standards, and ethos ranging across the different units.

Each unit and individual within each unit is required to have equal if the not the exact same capability as any other unit or individual within the Regiment. There is no exception to this. When one unit or individual fails to meet the exacting standards required of the Regiment they are re-trained, re-equipped, or replaced.

The rigorous selection process required prior to assignment to the Ranger Regiment, the intense training required to remain in the unit, and the requirement to remain in exceptional physical condition (no special considerations are given for age or disability) are the reasons this population has remained at what I have termed as "corps military homogeneous." This homogeneity has been true to this unit through the more than 25 years since the official activation of the 75th Ranger Regiment in 1986 (USASOC, 2011a, 2011b, 2012b).

Though the demographic characteristics varied somewhat across the different units with respect to those who volunteered to take the survey (i.e., study volunteers may have varied by age, rank, education, experience, MOS, and time in service, etc.), the study design as a convenience sample survey did not preclude any one demographic characteristic from taking precedence over any other.

Because of the known similarities and the so called "core military homogeneity" of the 75th Ranger Regiment population, this study was designed to measure the population as a whole and not compare differences between groups. Furthermore, the power analysis was performed on the basis that the Ranger Regiment was one group because the potential samples sizes from any one unit do not approach those necessary to legitimize any power 
analysis requirements. Additionally, the research questions were only posed to the population as a whole.

This will all be thoroughly examined in the Results and Discussions chapters, but was intentionally discussed in such detail here because of comments or conjectures that were made during the proposal phase of this research. These were made by uninformed university staff members who assumed that the Ranger populations, across the various locations, were too different to sample as one group and that generalizing any findings to the entire Ranger population as a whole would not be possible.

The Author holds firm to the assumptions that this population is very similar across all units and locations, and that the comments or conjectures made during the proposal phase were simply not well informed. This detailed descriptive, the results of the analyses, and the conclusions drawn from them will better inform these past suspicions.

\section{Demographic Data Estimates}

As previously discussed and for various security reasons, only verified estimates of the Ranger Regiment population are presented here. This estimated data did prove to be very similar to the actual Ranger participant population that volunteered for the study, as will be discussed in the results chapter.

Total 75th Ranger Regiment Population: +/- 3500

Total Population per Ranger Unit:

- Approximately 800-1000 assigned to each Ranger Battalion (1st, 2nd, and 3rd)

- Approximately 200 assigned to the Regimental Headquarters

- Approximately 350 assigned to the Special Troops Battalion

\section{Population of each Ranger Battalion, by Rank Status:}

Within a group of approximately $800-1000$ personnel that are assigned to the three major Ranger Battalions (not including the RSTB or RHQ), 720-900 are either Enlisted or Non- 
Commissioned Officers (NCO), 72-98 are estimated to be Commissioned Officers (O), and 4-8 are estimated to be Chief Warrant Officers (CW).

The rank status of those assigned to the RSTB and the RHQ varies tremendously by date and rotation of personnel in and out of these units. An educated estimate would conclude that $80 \%$ of all Officers assigned to both the RSTB and the RHQ are Captain (O3) or above, and $80 \%$ of all Enlisted personnel are NCOs at the rank of E6 or above.

Age:

The ages of personnel assigned to the 75th Ranger Regiment range from 18 years to 45 yrs old. There are some outliers that range above 45 yrs old, but the rugged operations tempo and demanding physical fitness requirement precludes many over this age to remain in this organization. Of this, roughly $80 \%$ are between $18-30$ years old and less than $10 \%$ are between the ages of $35-45$.

Education:

Every Ranger assigned to the 75th Ranger Regiment must either have a High School diploma or pass a recognized High School equivalency exam. These are very special people who aspire to be a part of this unit; therefore, multiple levels of education are seen sporadically throughout the population, in all ranks and experience levels. These range upward through Master's and Doctoral Degrees.

Gender:

This is an "all male" population. Army regulations preclude female assignment directly to the 75th Ranger Regiment.

\section{Ethnicity:}

The ethnicity within the 75th Ranger Regiment varies as personnel transfer in and out of the organization. There are no set requirements or directives that govern their population. It is strictly based on volunteer assignment and passing the basic training assessment process 
required for entry into the unit. Currently it is estimated that ethnic backgrounds are approximately: Black-10\%, Caucasion-69\%, Hispanic-20\%, and Other-1\%.

\section{Physical Fitness:}

All 75th Ranger Regiment personnel are extremely fit and healthy. They must pass rigorous physical fitness testing, conduct daily physical training, and pass detailed physical exams to both enter and/or remain in these units. All assigned personnel are considered physically fit or very physically fit by U.S. Army standards (USA HQ, 2010). Health:

As mentioned above, personnel assigned to these units pass detailed physical exams conducted at regular intervals in order to both enter and/or remain in these units. Their health is followed very closely by the medial personnel assigned to these units. Each person assigned is considered in either Good or Excellent physical and mental health, as considered by U.S. Army standards (USA HQ, 2007).

\section{Military Rank Groups:}

Ranger units consist of all U.S. Army Rank structures and levels that range from Private Enlisted level one (1) or E1 is the lowest entry level rank for an enlisted soldier. Colonel, which is a high level field grade commissioned officer rank, is measured as Officer level six (6) or O6. An entire explanation of the U.S. Military rank structure is not within the limits of this dissertation. In general, the rank structures groups within the Regiment include:

1. Enlisted Ranks comprise approximately $45 \%$ of the total Ranger population.
a. Private (pay grade E1 and E2)
b. Private 1st Class (pay grade E3)
c. Specialist and Corporal (pay grade E4),

2. Non-Commissioned Officers (NCO) Ranks again comprise approximately $45 \%$ of the total Ranger population. 

a. Sergeant (pay grade E5)
b. Staff Sergeant (pay grade E6)
c. Sergeant First Class (pay grade E7)
d. Master Sergeant and First Sergeant (pay grade E8)
e. Sergeant Major (pay grade E9)

3. Chief Warrant Officer Ranks comprise only about $1 \%$ of the total Ranger population.

a. Five ranks of Chief Warrant Officers (pay grade CW1-5)

4. Commissioned Officer Ranks comprise approximately $9 \%$ of the Ranger population.
a. 2nd Lieutenant (pay grade 01)
b. 1st Lieutenant (pay grade $\mathrm{O} 2$ )
c. Captain (pay grade O3)
d. Major (pay grade O4)
e. Lieutenant Colonel (pay grade O5)
f. Colonel (pay grade 06)

\section{Investigators}

Due to the complex negotiations that were required of the author to both access funding and gain access to the USASOC population of Rangers, the author (also the primary investigator) was required to accept the assistance of two co-investigators. In order to receive funding from the Military Infectious Disease Research Program (MIDRP) a collaborator from the Walter Reed Army Institute of Research (WRAIR) was required to collaborate on the project; therefore, Dr Paul Scott was accepted as a co-investigator and collaborator. In order to access the Ranger population, the leadership at the USASOC required an individual collaborator from within their organization to be an active member of the research time; therefore, Major Scott Gilpatrick was accepted as an assistant investigator and collaborator to this research. 


\section{Funding}

The study was funded by the Military Infectious Disease Research Program (MIDRP), executed via the U.S. Military HIV Research Program (MHRP) through the Department of Epidemiology and Threat Assessment of the WRAIR in Rockville, MD. The initial budget was left somewhat open but planned to be near $\$ 25,000$. The actual total cost was only $\$ 14,230.79$.

\section{Institutional Review Boards}

To insure the full protection of human subjects, this research was conducted in full compliance with all required Institutional Review Boards (IRB): there were two IRBs required for this research 1) the Florida International University (FIU), and 2) the WRAIR IRB. Details of the IRB process can be reviewed in Appendix 3.4

\section{Sample Size Calculations}

Initial power analysis calculations were conducted in February 2011utilizing G*Power version 3.1.2 (Faul, Erdfelder, Buchner, \& Lang, 2009) software using bivariate correlation and logistic regression analysis. The minimum sample size was determined to be 93; using a medium effect size, $80 \%$ power, an alpha of 0.05 , and a $10 \%$ overestimate. Maximum sample size was also calculated using a medium effect size and an alpha of 0.05 , but the power was set at $90 \%$. Including a $10 \%$ overestimate, this yielded 124 as the maximum sample size required. The maximum sample of 124 was the study goal at that time.

In October of 2011 new power analysis calculations were conducted, again utilizing $\mathrm{G}^{*}$ Power version 3.1.2 software with bivariate correlation and logistic regression analyses. The new calculations were to determine power analysis changes that may result from a maximum sample increase from 124 to 150 , which was being considered for IRB approval for at the time. The new calculations revealed that increasing the maximum sample size to 150 would improve the power analysis from $90 \%$ to $96.4 \%$ for bivariate correlations, and from $90 \%$ to $97.8 \%$ for logistic regression analysis for the outcomes, both of which were calculated at an alpha of 0.05 . 
IRB approval to increase the sample size to 150 and was granted and the new maximum sample size was the new recruitment goal. Please see Appendix 3.5 for the power analysis protocols.

\section{Data Collection Protocol}

Data collection was conducted via a cross sectional design utilizing the aforementioned survey questionnaire. The questionnaire was administered to a representative convenience sample of volunteers from each of the five 75th Ranger Regiment units. The author was present to proctor the administration of each survey.

The questionnaire was administered during normal duty hours, at the place of duty, which required very minimal effort from the volunteer participants. There were no identifiers collected. Each participant was given a large, sealable, nontransparent envelope, and asked to self-place and seal their questionnaire into that envelope prior to returning it. The Author then secured the enveloped questionnaires and insured their safe transport to the WRAIR data processing facility at Rockville, Maryland.

\section{Recruitment}

The relative secrecy, isolation, and high operation tempo of the Rangers required participant recruiting to occur as the opportunities presented itself throughout the various Ranger operating areas in and around each of the five Ranger unit locations. The initial goal was to recruit enough participants to meet the $90 \%$ power sample size of 124 successfully completed survey questionnaires, but after receiving IRB approval to increase the sample of as many as 150 participants; this became the ultimate recruitment goal and was achieved. All participation was $100 \%$ voluntary.

This initial proposal called for proportional sampling. Given the estimated population of the Ranger Regiment of 3500 and sample size of 150, the proportional sampling from each unit was easily calculated $4.3 \%$ of each population. Nevertheless, the rapid deployment capacity 
and high operational tempo of the Ranger units was ever changing during the data collection phase. At multiple times the Ranger units cancelled recruitment engagements and even expecting to deploy to Afghanistan at undetermined intervals. For this reason, oversampling became necessary to insure that at least the minimum sample size of 124 was met to achieve the $90 \%$ power from the initial sample size calculations. There was a real threat that the population may deploy to Afghanistan and reaching the $90 \%$ power would be impossible. Therefore, oversamples from Ranger Special Troops Battalion (RSTB), the Regimental Headquarters (RHQ) were taken, with the majority taken from $R H Q$.

After weighing the possibility of losing multiple units due to the threat of deployment and after careful consideration of the similarities of participants across the Ranger population, these oversamples were not considered a threat to the integrity of this study. For example, the majority of oversampling was from the $\mathrm{RHQ}$. Considering that the similarities previously discussed across the Ranger population, and that the majority of personnel assigned to the RHQ units had previously been assigned to a number of Ranger units; this was a reasonable practice. Similar considerations could be made for the RSTB, though it was not oversampled to the same extent.

Furthermore, this oversampling was not extreme. The original calculations for both the RSTB and the RHQ, considering $4.3 \%$ of those populations, were respectively 15 for the RSTB and 9 for the RHQ. The actual sampling totals from each of these units were 20 from the RSTB and 24 from the $\mathrm{RHQ}$; respectively this reflects an oversample of only 5 participants from the RSTB and 15 from the RHQ. The samples from the other units were relatively proportional.

Recruiting efforts took several forms and were all conducted onsite. These efforts included a range of tactics, from large recruitment briefings at the battalion, company, or platoon level, to small tailored briefings of groups as small as one to three individuals. At times the 
author even loitered around the Ranger unit areas recruiting and surveying volunteer's on-thespot, as the moment and time permitted.

Most recruitment activities were given to larger groups ranging from 10 to 30 personnel; therefore, the time period between actual recruitment and administration of the survey was only minutes. Some small groups and individuals were often recruited at one point and then the questionnaire was administered with several hours to a few days from that event.

Refreshments in the form of food and beverages were provided at each recruitment event. Beverages ranged from bottled water, to various sodas, juices, and energy drinks. Pizza was provided on the last day of each recruitment event, at each unit location. These refreshments were offered to anyone, even to non-volunteer personnel.

Recruitment of an ombudsman was never a valid option. Not only could I not find anyone outside the Ranger Regiment who would complete the required Human Subjects training, but the schedule changes to accommodate the high operations tempo of the Ranger population would not permit reliable forecast required for scheduling. The IRB did not require an ombudsman, but it was thought that during the initial planning it would be a valid option. It never materialized.

Recruitment Schedule. Recruitment efforts were conducted on Monday thru Friday between the hours of 9am to $4 \mathrm{pm}$ at the following locations, on the specified dates:

1. August 29th thru September 10th, 2011 at the Regimental Headquarters, the Regimental Special Troops Battalion, and the 3rd Ranger Battalion located at Fort Benning, Georgia.

2. October 2nd thru October 12th, 2011 at the 2nd Ranger Battalion located at Ft Lewis, Washington

3. November 13th thru November 18th, 2011 at the 1st Ranger Battalion located at Hunter Army Airfield located in Savannah, Georgia. 


\section{Inclusion Criteria}

Participants were required be 18 years old, male (this is an all male unit) and assigned to one of the Ranger units. Participants also must have also either prepared for or traveled to at least one malaria endemic region of the world within the past 36 months.

\section{Exclusion Criteria}

Participants who had allergies, contraindications, or suffered from any adverse reactions that precluded them from taking malaria chemoprophylaxis medications were excluded.

\section{Informed Consent}

All participants were given an anonymous and unsigned informed consent form and briefing to complete prior to administration of the questionnaire. The form was read aloud to each group of volunteers. The form did not require a signature, but did require each participant acknowledge they understood this informed consent by reading and answering the following questions:

Do you fully understand the purpose, duration, and procedures of the study? Yes / No Do you fully understand the risks, benefits, and alternatives to participation? Yes / No Do you understand your participation is completely voluntary? Yes / No Do you understand you may withdraw at any time? $\quad$ Yes / No Do you consent to participate by completing this questionnaire? $\quad$ Yes / No Are you 18 years old or older? Yes / No

Any participant who responded with a "no" to any question was not allowed to participate in the study and/or complete the questionnaire. The anonymous consent form can be reviewed in Appendix 3.5. The completed forms are kept at the WRAIR Data Coordinating and Analysis Center (DCAC). 


\section{Analysis Plan}

Quantitative data have been imported from the questionnaire format and manually double entered, with appropriate quality controls, by the WRAIR DCAC staff into a ClinPlus@ data base. Please see Appendix 3.3 for the DCAC Data Management Plan details concerning the development of this database including: data flow, database specifications, database testing, data entry and processing, data validation, quality controls, security measures, database lock/freeze, and questionnaire archiving. To maintain full integrity of the database, the entire database was created solely by DCAC personnel under their management oversight and governing procedures.

After the database was created and appropriately tested, as outlined in the DCAC Data Management Plan, the data was then transferred to the author via the WRAIR secure data transfer system. The database was downloaded and maintained within a password protected file. The data has been maintained and backed up within a password protected external hard drive as well. All statistical analyses were conducted by the author using SPSS@ Version 20.0, predictive analytical software.

\section{The First Null Hypothesis}

In order to accept or reject the first null hypothesis $\left(\mathrm{H} 1_{\mathrm{O}}\right.$, No relationship exists between the current KAP domains, environmental domains, and behavioral outcomes.), Bivariate Pearson Correlations were conducted to test for significant relationships between both KAP and environmental variables, and behavior outcomes. Any significant relationship noted were based on an alpha of 0.05 (Faul et al. 2009; Fox, 2008).

\section{The Second Null Hypothesis}

In order to accept or reject the second null hypothesis $\left(H 2_{O}\right.$, The current KAP domain variables cannot predict behavioral intentions, as a proxy for behavior change.), binary logistic regressions were used to determine any predictive relationships between independent variables 
and the dependent variables (Faul et al. 2009; Fox, 2008; Kleinbaum, Kupper, Muller, \& Nizam, 1998). Any significant effects were based on an alpha .05. Odds ratios were interpreted, considering the $95 \%$ confidence intervals, regarding predictive potential of the independent variables relative to the outcome or dependent variables. The binary logistic regressions were first analyzed to identify significant independent variables within each domain of each behavior.

The significant variables from each of the eleven domains were then entered into an overall model that combed all these variables within that behavior. A combined model was created for each behavior: malaria medications, condom use, and weapon safety.

\section{The Third Null Hypothesis}

In order to accept or reject the third null hypothesis $\left(\mathrm{H}_{\mathrm{O}}\right.$, Command Support domain variables do not moderate the relationship between variables within the KAP domains of perceived susceptibility and risk, behavior related practices, interpersonal communications, and the behavior outcomes.), the Moderated Logistic Regression Analysis (Sharma, Durand, \& GurArie, 1981) process within the SPSS(C) Version 20.0, predictive analytical software. This process was used to identify potential significant interaction effects of independent variables within a binary logistic regression equation. Any significant interaction effects were based on an alpha .05 . Odds ratios were then interpreted, considering the $95 \%$ confidence intervals, regarding predictive potential interaction effects of any potential moderator variables relative to the outcome or dependent variables.

\section{Overview of the Analysis Process}

Organizing the Database for Analysis. The database was received via the Secure File Transfer (SFT) system on January 06, 2012. The file was transferred in a zip format. When opened the file contained 35 individual SPSS $®$ data sets, each representing one domain of the questionnaire (33 domains), including a file with the three Administrative questions and another that contained the Demographic variables. 
These 35 data sets were then consolidated into four data sets that would facilitate appropriate data analysis. The data from the original database transfer was not altered in any form. The four newly created data sets included:

1. Demographics data set containing 17 Demographic variables.

2. Malaria Chemoprophylaxis data set containing 108 variables.

3. Condom Use data set containing 109 variables.

4. Weapon Safety data set containing 104 variables.

The three administrative questions remained in a separate file. These questions were not relevant to any independent or dependent variables and were therefore not included in any analyses. These questions are named below:

1. About how much time did it take you to complete this survey?

2. Can you please tell us if any questions offended you?

3. If any questions did offend you: Please tell us which ones.

Prior to any analysis, the questionnaire was scrubbed for any overt issues that might not have been identified during the development of the questionnaire and that may preclude further analysis.

The first of these issues was that some of the Knowledge questions/variables could not be "graded" per se, as being correctly responded to or not; therefore, a decision was made to subdivide the Knowledge questions from each behavior into two categories, a gradable category, and a general knowledge category.

The gradable category of knowledge variables included:

1. Malaria variables included 11 variables. Examples are:

a. Can a person get malaria from a mosquito bite?

b. Can a person get malaria from blood transfusions? 
c. Can malaria be transmitted from person to person by close contact or cough?

2. Condom variables included 13 variables. Examples are:

a. Can a person get Sexually Transmitted Infections (STIs) from improper use of condoms?

b. Can a person get some of the same STIs from blood transfusions?

c. Can STIs cause death?

3. Weapons variables included 10 variables. Examples are:

a. Which is "not" a correct performance step in zeroing the M4 or M4A1 Carbine?

b. Which is "not" a correct performance step in unloading the M4 or M4A1 Carbine?

c. During Urban Operations: Is it safe to shoot while moving faster than you can engage your targets?

The remaining variables were either dropped or included in the General Knowledge category. Each of the sub-categories was entered into the data analysis process in the same manner as the other variable described in this analysis plan.

The Dependent Variables. Two dependent variables were identified for each of the three health behaviors (malaria medications, condom use, and weapon safety). The first dependent variable (listed in item 1 below) measured the actual behavior relevant to whether it was or was not performed by the survey participants in the past. The second dependent variable (listed in item 2 below) measured the behavioral intentions of each survey participant, relative to the next time they performed the behavior. The specific dependent variables are listed below. 
1. The dependent variables measuring the actual behavior from the three behaviors are:

a. Take Meds as Directed - When you yourself were prescribed malaria chemoprophylaxis medications: Did you take them exactly as directed?-Referred to from this point on as Take Meds as Directed. This variable was represented in the questionnaire by question M23A.

b. Use a Condom - Outside a long-term and strictly monogamous relationship: Did you yourself use a condom during every act of sexual intercourse?--Referred to from this point on as Use a Condom. This variable was represented in the questionnaire by question C34A.

c. Follow Weapon Safety - Do you yourself follow the recommended weapon safety guidance at all times?--Referred to from this point on as Follow Weapon Safety. This variable was represented in the questionnaire by question W45A.

2. The dependent variables measuring the intention to follow next time from the three behaviors are:

a. Intend to Take Meds Next Time - Do you plan to take your malaria chemoprophylaxis medications exactly as directed the next time?--Hereto referred to from this point on as Intend to Take Meds Next Time. This variable was represented in the questionnaire by question $\mathrm{M} 23 \mathrm{H}$.

b. Intend to Use Next Time - Do you plan to use a condom the next time you have sexual intercourse outside a strictly monogamous relationship?-Hereto referred to from this point on as Intend to Use Next Time. This variable was represented in the questionnaire by question C34I. 
c. Intend to Follow Next Time - Do you plan to follow the recommended weapon safety guidance the next time you operate a weapon?--Hereto referred to from this point on as Intend to Follow Next Time. This variable was represented in the questionnaire by question $\mathrm{W} 45 \mathrm{H}$.

Independent Variables. Identifying these six (6) questions as dependent variables further reduced the questions that could be analyzed as independent variables. There were now 332 potential independent variables. There were no skip questions built into the questionnaire; therefore, each of the remaining 332 questions had the potential, as per the analysis plan, to be a significant independent variable.

Selecting Variables for Analysis. Each of the 332 variables indentified as independent variables was equally based on the theoretical constructs. After hours of contemplation and consideration, no question was determined as more important, or more theoretically significant than any other. Therefore, the decision was made to use Chi-square statistics to show the association between independent variables and the dependent variables.

Frequency tables were first run on every variable. Crosstabs with Chi-square statistics were then conducted on every independent and dependent variable. Those independent variables found to have statistically significant relationships $(\alpha \geq 0.05)$ with the dependent variables were selected for further analysis through bivariate Pearson correlations, binary logistic regression, and moderated logistic regression (interaction variable) analysis.

Two exceptions were made reference the significance requirement:

1. The demographic variables that represented unit of assignment and age did not have significant relationships with malaria or weapon dependent variables. Despite the lack of a significant relationship, these variables were entered into both bivariate Pearson correlations and binary logistic regression models. These variables were both entered further analysis because 1) unit of assignment was considered important to examine in order to explore the 
homogeneity of this population, and 2) though age (aside from advanced age) has not been found in the literature to affect adherence; in military populations, age is often a reflection of experience, which may imply a higher level of operant conditioning as noted in the TRA/TPB.

2. The Independent variables within the Knowledge domains had very few significant relationships with the dependent variables, using Chi-square statistics. To avoid criticisms of under analyzing this domain, the decision was made to include them all into further analyses.

Estimates of Internal Consistency Reliability Analysis of the Domains. Prior to any detailed analysis through bivariate Pearson correlations and/or binary logistic regression models; an internal consistency reliability analysis with Cronbach's Alpha was conducted on each domain, resulting in 33 Cronbach's Alpha coefficients. Twenty two of the 33 coefficients were at 0.70 or greater (Table I).

The general standards for acceptable reliability are 0.700 or greater (Ferketich, 1991; Nunnaly \& Bernstein, 1994); therefore, the internal consistency reliability of $67 \%$ of the measures were reasonably reliable for interpretation in more detailed analyses, particularly when combining or aggregating scores.

The scales within the domains with coefficient scores below 0.70 are not considered internally consistent and may not reveal meaningful information. When examining the total-item statistics within the Cronbach's Alpha analysis; some manipulations can be conducted by removing variables from this analysis to improve the Cronbach's Alpha coefficient scores. These manipulations will be further examined and considered if any significant results are found in the bivariate Pearson correlations, or binary logistic regression analyses. 
Table 3.I. Cronbach's Alpha Coefficients for KAP and Environmental Domain Measures $(n=150)$

\begin{tabular}{|llll|}
\hline KAP Domains : & Malaria & $\underline{\text { Condom }}$ & Weapons \\
1. Behavior Related Knowledge & 0.723 & 0.558 & 0.384 \\
2. General Behavioral Attitudes & 0.746 & 0.735 & 0.603 \\
3. Descriptive Norms & 0.579 & 0.971 & 0.922 \\
4. Injunctive Norms & 0.818 & 0.916 & 0.948 \\
5. Perceived Susceptibility and Risk & 0.125 & 0.454 & 0.501 \\
6. Behavior Outcome Related Practices & 0.765 & 0.854 & 0.291 \\
7. Interpersonal Communications & 0.828 & 0.556 & 0.785 \\
Environmental Domains: & & & \\
8. Behavioral Related Education & 0.869 & 0.770 & 0.874 \\
9. Behavior Related Training & 0.767 & 0.367 & 0.790 \\
10. Behavior Related Media & 0.802 & 0.803 & 0.926 \\
11. Command Support & 0.714 & 0.633 & 0.759 \\
\hline
\end{tabular}

Transforming and Cleaning Variables for Analysis. In order to effectively analyze the variables within bivariate Pearson correlations and binary logistic regression models, several data transformation processes were implemented to both the dependent and independent variables within the datasets. The data transformation was necessary to avoid the SPSS ${ }$ binary logistic regression program from creating unnecessary dummy variables, which produced misleading results.

The data transformation included dichotomizing the dependent variables, excluding certain response choices from further analysis equations and models, as well as creating ever/never versions of certain domain variables. No data was added or removed from the 
original database created by the WRAIR DCAC personnel. Details of the data transformation process included the following:

1. The dependent variables for each behavior were dichotomized.

These variables were dichotomized into the binary forms of yes (coded as 1) and no (coded as 0). This dichotomization was accomplished by excluding response choices that would either not improve a correlation or regression model, and/or those responses that could not be definitively and positively justified to have the potential to be changed to a Yes or No response.

Responses don't know, not applicable, and refuse to answer were excluded from further analyses.

2. A similar process was conducted on the independent variables.

Responses that did not definitively add to the model, and/or those that could not be definitively argued as belonging to or being combined with a more definitive responses (e.g. yes, no, somewhat likely, very likely, most of the time, some of the time, etc.) were not included into any analysis equation or model. These responses were transformed into dummy variables by SPSS $®$ predictive analytic software during the binary logistic regression analysis process.

Responses such as: other, don't know, not applicable, and refuse to answer were choices that were not included.

3. The next process was to create ever/never variables within the domains of behavior outcome related practices, interpersonal communications, and command support.

The ever/never variables were dichotomized: ever was coded as 1 , and never was coded as 0 . This process was identical to that used to dichotomize the dependent variables. 
These ever/never variables were analyzed through Pearson correlation and binary logistic regression equations, just as any other independent variable was analyzed. These variables would further illustrate the predictive potentials in comparison to controlling for the multiple parameter codes of the same variables other full version variables.

Identification of the Newly Transformed Variables. New Variables were created within the database and given appropriate names that reflected the changes made during the data transformation process. Additional identification codes were added to the names and labels of each modified variable inside the database that signified the changes. These names are very obvious and are easily noted when reviewing the data sets or output information.

The additional identification codes include the following:

1. The letters $w D s$ were added to the names and labels of those new variables that were modified to exclude the aforementioned responses. These letters $w D s$ stand for with drops. This data cleaning process, adding with drops, is only a metaphor to signify that responses were excluded from analysis. No data was actually removed from the data base. Variables were simply given new names to signify the exclusion of responses. For example, the new name for question M23G was now M23G.wDs.

2. Similarly, the letters $D w D s$ were added to the names and labels of those variables that were both dichotomized and modified to exclude the aforementioned responses. The letters $D w D$ s stand for dichotomized with drops, again a metaphor. For example, the new variable name in the database changed from question M23G to M23G.DwDs after being dichotomized and modified to exclude the aforementioned responses. 
3. For the newly created ever/never variables, the phrase ever.never was added to the names and labels of those new variables. Since these variables were also dichotomized and modified to exclude unnecessary response choices, they also included the letters $D w D$ s as explained above. An example of a new ever/never variable name created from question M23G is M23G.wDs.ever.never.

These codes are only utilized within the database and SPSS $®$ data output. Codes are often hard to remember and confusing to readers; therefore, when significant variables are identified and discussed from this point on (including Chapters IV and V), only names will be used. For example, the name for M23G.wDs is Plan malaria meds into missions.

\section{Details of Analytical Techniques}

Though cross sectional survey data lacks the power to infer cause and effect, they are considered powerful enough to predict behavioral intentions, as a proxy for behavioral change.

This study has utilized bivariate correlations, binary logistic regressions, and moderated regression analyses. The final interpretations fully discussed in the Results chapter; nevertheless, this final section will explain the details of these analyses. All predictive probabilities of the independent variables are also based on the dependent variables membership as Yes.

The significant bivariate correlation, binary logistic regression, and moderated logistic regression analysis results associated with any analysis of the dependent variables Take Meds As Directed, Use Condom, and Follow Weapon Safety are not sufficient, considering the cross sectional design, to infer cause and effect relevant to the behavioral outcomes. Nevertheless, these outcome related dependent variables were selected because they were anticipation to be helpful at 1) Informing military commanders, public health, and medical personnel on the current levels their military members are following this health related behavior, and 2) Guiding future interventions to improve behavior outcomes. 
Alternatively, the significant bivariate correlation, binary logistic regression, and moderated logistic regression analysis results associated with the dependent variables Intend to Take Meds Next Time, Intend To Use Next Time, and Intend To Follow Next Time are specific to the null hypotheses supporting this research and they are intended to predict behavioral intentions, as a proxy for behavioral change. Nevertheless, these findings are also anticipated to be very helpful at 1) Informing military commanders, public health personnel, and medical personnel on the current behavioral intentions relevant to these KAP and environmental domain variables, 2) Predicting behavioral intentions, and 3) Guiding future interventions to improve behavior outcomes.

Bivariate Pearson correlations. Bivariate Pearson correlations were conducted on every independent variable with a significant Chi-square relationship to one of the three dependent variables.

Only significant medium $(-0.5$ thru -0.3 and +0.3 thru +0.5$)$ and strong $(-1.0$ thru -0.5 and 0.5 thru +1.0) correlation coefficients will be reported (Buda \& Jarynowski, 2010; Cohen, 1988). An exception was given to some domains that had only low correlation coefficients that were $\geq \pm 0.2$ available to represent the relationship between that domain and the dependent variables.

Binary logistic regression. Binary logistic regressions were conducted on every independent variable with a significant Chi-square relationship to one of the three dependent variables.

Forward stepwise, conditional method. The SPSS $₫$ software offers several different choices of entering data into a Bivariate Logistic Regression Equation. All choices were thoroughly explored and the most appropriate data entry selections were chosen.

Due to the exploratory nature of this study (Kleinbaum et al., 1998; Zelterman, 2009) and the difficulties previously discussed reference the direct theoretical applications; binary logistic 
regression calculations were entered using the Forward Stepwise, Conditional with Indicator first method. This section will further elaborate on how this selection was justified.

The forward stepwise, conditional entry method begins with no variables in the model and then enters them into the equation utilizing a stepwise variable entry based on significant chi-square statistic scores (Kleinbaum et al., 1998; Zelterman, 2009). The variables are then removed based on their likelihood ratio score, given conditional parameters (Kleinbaum et al., 1998; Zelterman, 2009). Conversely a similar backward entry method would begin with all variables in the model and then removes them similarly.

The Conditional parameter method was chosen, vs. the Likelihood Ratio (LR) or the Wald methods, because of the fact that Conditional parameters are based on exact conditional estimates rather than Maximum Likelihood estimates; as is the case with the LR method (Hess, 2010; Kleinbaum et al., 1998; McCarthy \& Guo, 2009). Additionally, maximum likelihood estimates do not exist in many databases (McCarthy \& Guo, 2009). Therefore, the conditional parameter method was thought to be the best option for producing more exact and unbiased predictive estimates from the logistic regression analysis with this database and population.

The Forward Selection, Wald method was not considered an option because of its preferred use on large sample population analyses (Fox, 2008; Kleinbaum et al., 1998; Zelterman, 2006), as was not the case in this study or 150 participants.

There are similar issues with both forward and the alternative backward selection process. The forward entry process may lead to under fitting the data by under selecting variables for entry into the model. On the other hand, the backward entry process may lead to over fitting the model with variables by initially entering all variables and not removing them (Kleinbaum, 1998; Zelterman, 2006).

Under fitting was not considered an issue in this analysis because of five control measures that were applied to this analysis plan. These controls included: 
1. Independent Variables were fist identified by significant Chi-square relationships to the dependent variables.

2. The highest number of variables entered into any forward entry equation was twelve. Most entries included only four to six variables.

3. Any forward stepwise selection equations that failed to conditionally select significant variables or that were found to have significant main effects but the non-significant parameter were re-analyzed through the enter method, which forces each variable into the equation.

4. Any border line or near significant results were also re-analyzed through the enter method.

5. If further analysis through the enter method improved the significance of the main effects, yet the remaining parameter codings were not improved, the indicator was changed from first to last and then entered into the equation again.

This step five (5) was only utilized in on instance, with M21J, which then yielded significant results. Otherwise, all other entries were Indicator first.

Indicator first. The indicator first method was the most reasonable option for nearly all variables in the database because the survey instrument and database were constructed with the most negative response to each question/variable as the first categorical response, coded as zero. Positioning the most negative response in the first position and coding it zero made the indicator first the most appropriate choice as the reference variable, particularly since it was compared to a yes dependent variable coded as one.

The moderated logistic regression. The moderated logistic regression analysis maintained the same forward stepwise, conditional with the indicator first entry method. Selecting the potential interaction/moderator variables was a two-step process. 
Step-one was to identify which Command Support domain variables would be entered into the moderated logistic regression equation as interaction variables. This was conducted by selecting those Command Support variables that had either a significant Chi-square or binary logistic regression relationship with the dependent variables from each of the three behaviors.

Once a significant relationship between a Command Support variable and an outcome variable was identified, that specific Command Support variable was only entered into a moderated logistic regression analysis with that specific dependent variable. For example, malaria Command Support variable How often can you recall your Command reinforcing malaria medication behavior (represented by question M28A1 in the questionnaire) was found to have a significant Chi-square relationship with the dependent variable Intend to take meds next time. Therefore, the variable How often can you recall your Command reinforcing malaria medication behavior, would only be used as an interaction/moderator variable in models that included Intend to take meds next time as the dependent variable.

The second step was to determine which independent variables from the domains of Perceived Susceptibility and Risk, Behavior Outcome Related Practices, and Interpersonal Communications would be entered into the model.

To maintain the analysis format and integrity, the same parameters used for entering independent variables into the bivariate logistic regression models were used for entry into these moderated logistic regression models. In other words, the variables that had significant Chi-square relationships with one of the dependent variables were selected for entry in an equation with that variable. For example, within the domain of Perceived Susceptibility and Risk, there were three variables that had significant relationships with the dependent variables Take Meds As Directed and Intend to Take Meds Next Time. These three variables were entered into the moderated logistic regression equations and include: 
1. Do you believe malaria is a serious disease?

2. Do you feel that malaria is a disease that only others will contract?

3. When you are in a region that has malaria: Do you think about malaria on a regular (daily or weekly) basis?

These and any other variables that met the criteria for this two-step process were entered the moderated logistic regression equations models as provided by the manual of instruction and tutorial applications provide by SPSS $尺$.

The combined model. The final step in the analysis plan was to combine all significant binary logistic regression variables from each of the eleven domains within each behavior into an overall combined binary logistic regression model. These combined models will also contain any significant moderator variables identified in the moderated logistic regressions. These models will identify the most significant predictive relationships between the independent variables and each behavior outcome. They were analyzed with the same forward stepwise process as all other logistic regression models.

There are two types of combined models, 1) the full combined model and 2) the ever/never combined model. The full combined models contain the each categorical response possibilities that were retained for analysis, as previously described. These responses are referred to later in the results and interpretations as dummy variables. The dummy variables are created by SPSS $₫$ when these categorical responses are entered into the binary logistic regression. The ever/never combined models have been dichotomized into only ever and never responses, as previously described.

Overall models were created for each of the three behaviors, but only those models relative to malaria chemoprophylaxis medication adherence are reported in this dissertation. Other data and/or information will be available upon request sent to the author at cmbtdvr@hotmail.com. 


\section{Testing the Homogeneity of the Population}

Defining or identifying the homogeneity of the Ranger population was never a consideration within the research questions supporting this research because the author has known them to be a homogeneous group. Nevertheless, there have been some comments to the contrary of this Ranger homogeneity. To illustrate the lack of any significant differences between the responses submitted by any one unit within the 75th Ranger Regiment, a one-way analysis of variance (ANOVA) was conducted. This one-way ANOVA is intended to observe any differences between the five Ranger units relative to the significant findings within the overall combined models of the analysis (Kleinbaum et al., 1998).

The one-way ANOVA will not be conducted on every significant variable and is only intended as an illustration of homogeneity. For the purposes of this illustration, the ANOVA will only be conducted on the most significant combined overall model variables found to potentially predict behavioral intentions relative to adherence to malaria chemoprophylaxis medications.

Because the sample sizes between the five Ranger units are not equal and to make comparisons of any contrasts or unequal variances that may be suspected between these units, the Scheffe's method will be the primary post-hoc multiple comparisons test (Kleinbaum et al., 1998). If the one-way ANOVA null hypothesis (i.e., there are no differences between groups) is retained, an additional Bonferroni post-hoc analysis will also be performed to insure a thorough application of the multiple comparisons testing is applied to this analysis.

The Bonferroni method is one of the most highly utilized and flexible post-hoc multiple comparisons testing methods (Kleinbaum et al., 1998; Olejnik, Li, Supattathum, \& Huberty, 1997) and might be considered suitable alone as a multiple comparisons post-hoc analysis. Since the unit populations varied, and given that one-way ANOVA will only be run on a limited number of variables within this complex dataset; conducting two post-hoc analyses (Scheffe's and Bonferroni) is anticipated to add the integrity of this limited analysis. 


\section{Cataloging of Significant Data}

Significant SPSS $®$ output data will be cataloged in and made available upon request sent to the author at cmbtdvr@hotmail.com. These significant data catalogs contain all significant data that was not included in this dissertation.

\section{Transparency}

All data and significant findings from this study will be shared with the USASOC, the U.S. Army Public Health Command, and the Walter Reed Army Institute of Research. 


\section{CHAPTER IV}

\section{RESULTS}

\section{Opening Statement}

Adherences to malaria chemoprophylaxis medications in U.S. military populations, including the Ranger population, are notoriously low. The reasons these personnel choose not to take these preventive medications properly are not clear. No studies exist that thoroughly examine the relevant KAPs associated with this behavior in the Ranger population. This exploratory study will help to fill this gap in the current knowledge and also inform future research and/or interventions aimed at improving adherence to malaria chemoprophylaxis medications in the Ranger population, as well as throughout the military. The U.S. Army Public Health Command is currently awaiting the completion of this study in order to inform and plan future research and/or interventions based on these results.

This chapter presents the results of this study, including 1) a detailed description of the population, 2) the reported levels of adherence relative to the outcome variables, 3) results of analyses that effectively reject the three Null Hypotheses supporting this research, 4) an illustration of study participant knowledge relative to the three behaviors, and 5) the results of limited one-way analyses of variance supporting the homogeneity of the Ranger study population responses.

\section{Description of the Participants}

The initial recruitment goal was to sample each unit proportionally relative to the total population of Rangers and the maximum sample size of 150 , which would equate to approximately $4.3 \%$ of the total estimated Ranger Regiment population of 3500 . The initial goal was to sample $4.3 \%$ from each of the five Ranger units. The operational tempo, deployment cycles into combat zones, and available time to sample did not afford this exact proportional sampling of $4.3 \%$. Rather, the Regimental Headquarters (RHQ) and the Regimental Special 
Troops Battalion (RSTB) were somewhat over sampled as explained in Chapter III. Nevertheless, the maximum sample size of 150 was achieved and each unit was well represented with study participants.

The over samplings of the RHQ and the RSTB were relatively small and respectively represented only 15 participants above the initial goal for the RHQ and 5 participants above the initial goal for the RSTB. The total number of participants recruited from the RHQ included 24 participants, versus the initial $4.3 \%$ proportional estimate of nine (9). The total number of participants recruited from the RSTB included 20 participants, versus the $4.3 \%$ proportional number 15.

Recruitment from the three Ranger Battalions (1st, 2nd, and 3rd) were sampled relatively proportional, with the 3rd Ranger Battalion yielding the least number of participants. The number of participants from the 3rd Battalion totaled only 28, which was slightly under the initial $4.3 \%$ estimate of 43 . The other two Ranger Battalions (1st and 2 nd) rather closely resemble the initial $4.3 \%$ estimates; with 1 st Battalion yielding 40 participants and 2nd Battalion yielding 38 participants. Figure 4.1 illustrates how closely the study participants resemble the actual population estimates. 
Figure 4.1: Estimated Proportion of Ideal Participants vs. Actual Study Participants from each

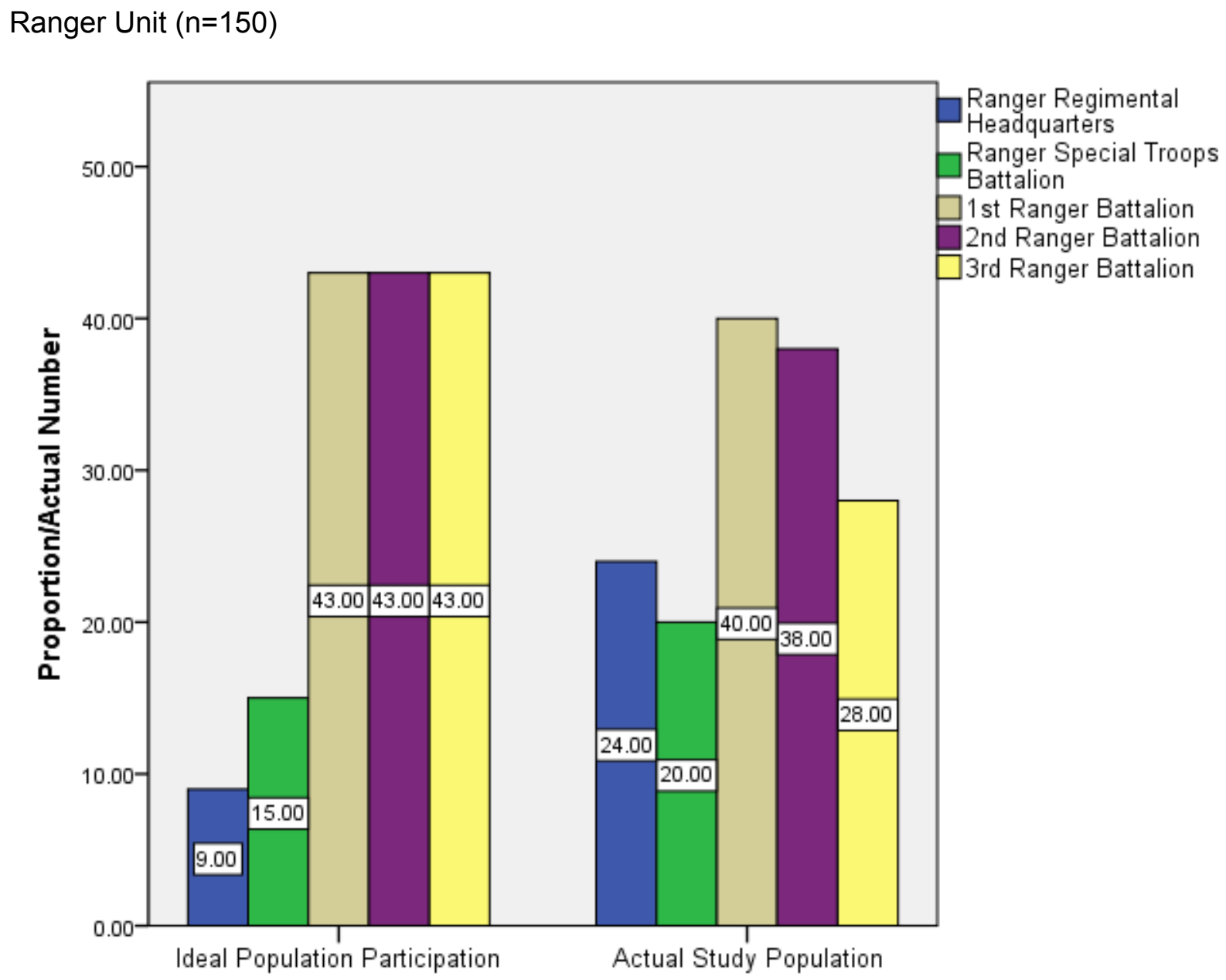

The number of actual participants does not exactly mirror the ideal proportions of the population that were described in the Chapter III at $4.38 \%$, but there is a reasonable representation of each unit. The details of the over and under sampling differences were also discussed in detail in Chapter III. 
Despite the limited differences between the initial goals of recruiting $4.3 \%$ of each unit population, those participants who were recruited appear to closely resemble the demographic estimates of the Ranger population with respect to multiple demographic parameters; including age, rank, and ethnicity. Figures 4.2, 4.3, and 4.4 illustrate the similarities between the estimates of the Ranger population and those of the actual participants relative to age, rank, and ethnicity.

Other demographic parameters were measured by the survey instrument, but there were no available demographic estimates from the Ranger personnel records to compare them in any detail and report in these results. 
Figure 4.2: Percent Estimate of Ranger Population Age vs. Actual Study Participants $(n=150)$

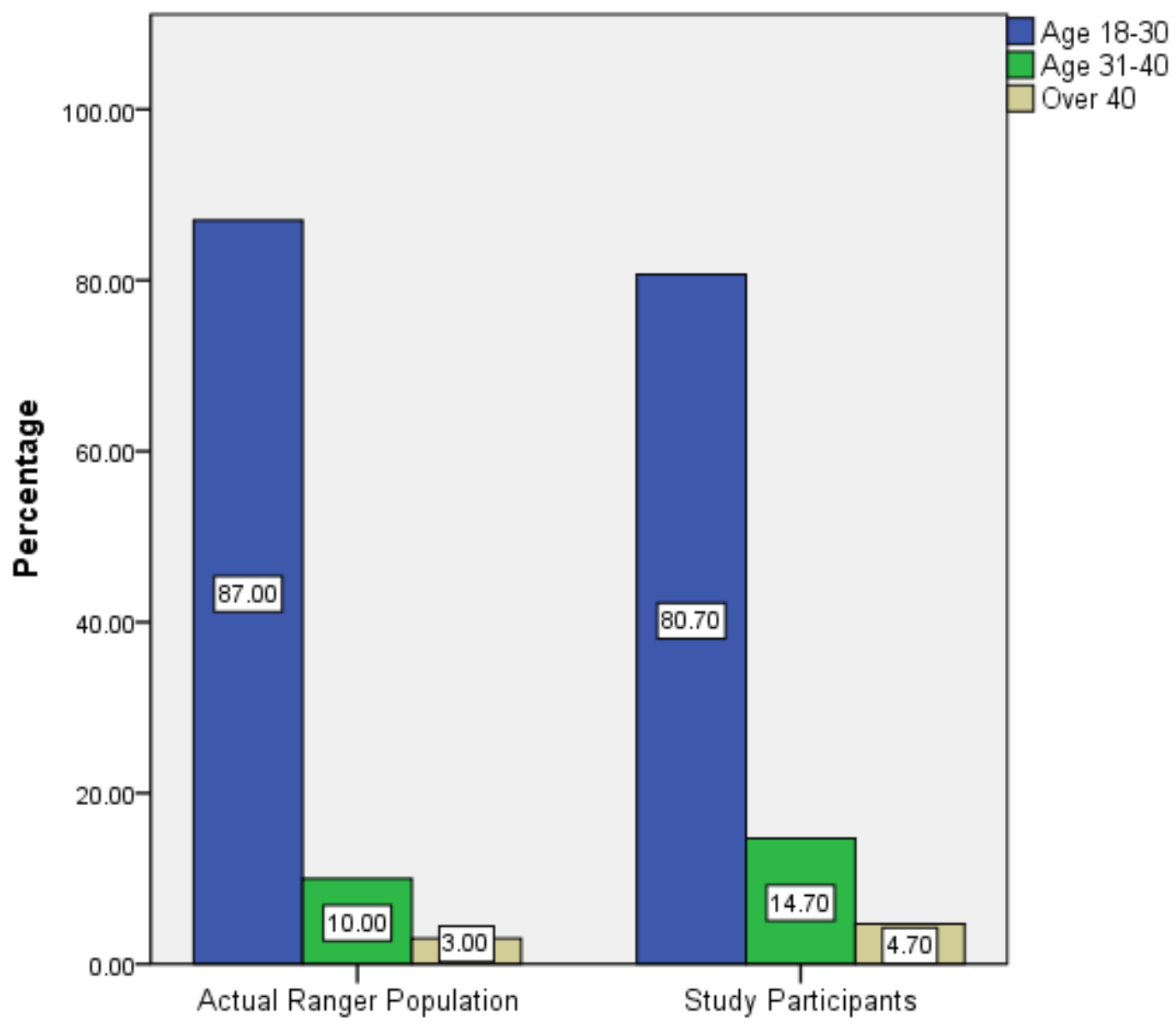

As noted in Figure 4.2, the ages of the actual study participants closely resemble those of the Ranger population estimates. 
Figure 4.3: Percent Estimate of Ranger Population Rank Structure vs. Actual Study Participants $(n=150)$

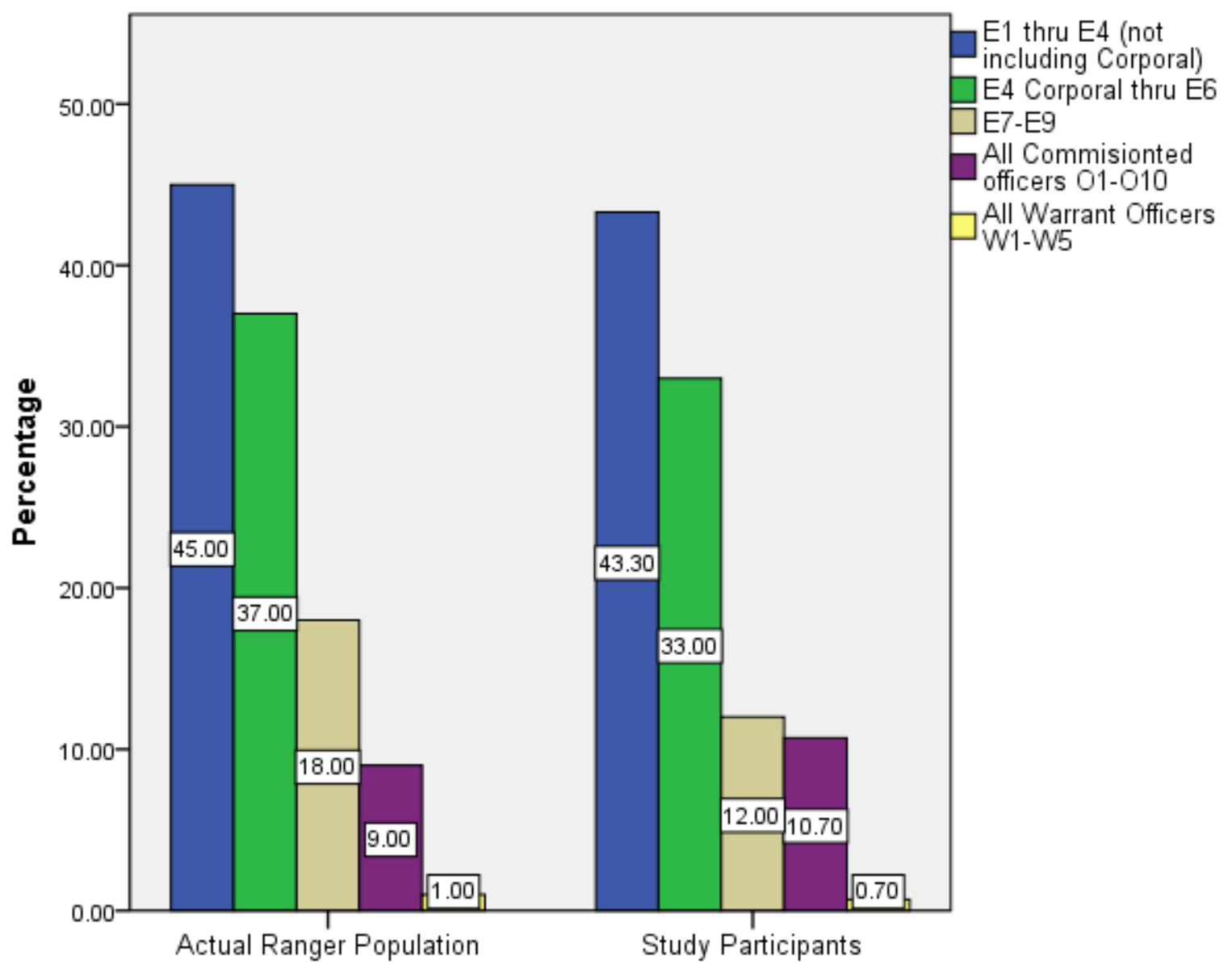

Figure 4.3 illustrates that the rank structure of the actual study participants closely resemble those of the Ranger population estimates. 
Figure 4.4: Estimated Percent Ethnicity of Ranger Population vs. Actual Percent of Study Participants $(n=150)$

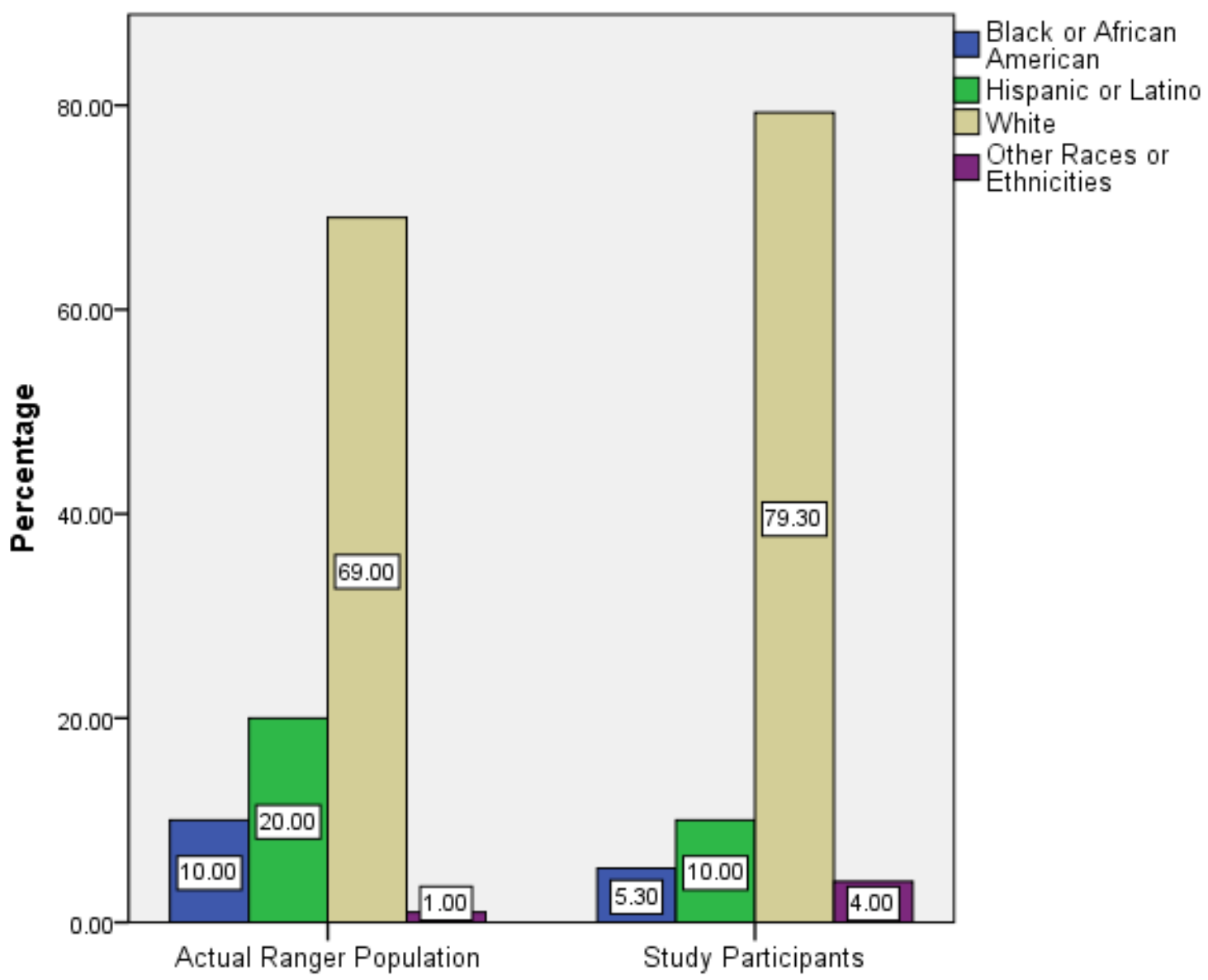

Figure 4.4 illustrates that the percentages of the given ethnicities within the actual study participants closely resemble those estimated within the Ranger population.

\section{Levels of Adherence and Intentions to Adhere}

In order to effectively discuss or illustrate any of the findings relative to the three behaviors this study focused on, the basic levels of adherence must be illustrated. The study measured both the actual adherence levels (i.e., took meds as directed, used a condom, and followed weapon safety), as well as the intentions to adhere next time. 
Adherence to malaria chemoprophylaxis medications in the form of taking these medications as directed at all times was found to be $32.9 \%(n=150)$ with the study participants. The intention to take the medications the next time was $60.8 \%(n=150)$.

Relative to condom use, adherence in the form of using a condom at all times during intercourse was found to be $10.1 \%(n=150)$ with the study participants. The intention to use a condom the next time was $52.7 \%(n=150)$.

Adherence levels concerning weapon safety were quite high in both cases with the study participants. Adherence in the form of following weapon safety at all times was $98.6 \%(n=150)$. The intention to follow weapon safety the next time had an adherence of $97.3 \%(n=150)$.

\section{Research Questions}

This study focused on answering three research questions:

1. What relationship, if any, exists between the current KAP domains, environmental domains, and behavioral outcomes?

2. Can any of the behavioral domain variables predict behavioral intentions, as a proxy for behavior change? Behavioral intention is the best predictor of behavioral change (DiClemente, Crosby, \& Kegler, 2009; Edberg, 2007).

3. Do variables within the environmental domains of behavioral education, behavior related training, behavior related media, or military command support moderate the relationship between any KAP domain variables? 


\section{Null Hypotheses}

The three research questions were subsequently transformed into the following Null Hypotheses:

$H 1_{\mathrm{O}}$ : No relationship exists between the current KAP domains, environmental domains, and behavioral outcomes.

$H 2_{0}$ : The current KAP domain variables cannot predict behavioral intentions, as a proxy for behavior change.

H3o: Command Support domain variables do not moderate the relationship between variables within the KAP domains of perceived susceptibility and risk, behavior related practices, interpersonal communications, and the behavior outcomes.

\section{Rejecting the Null Hypotheses}

\section{The First Null Hypothesis}

In order to accept or reject the first Null Hypothesis, bivariate Pearson correlations were conducted to identify any significant linear relationships exist between the either the KAP or environmental domain variables and the behavior outcomes. Multiple bivariate Pearson correlations were performed. The significance was based on an alpha of 0.05 . Only those significant relationships associated with adherence to malaria chemoprophylaxis medications are reported in this chapter. Nevertheless, a listing the significant bivariate Pearson correlation coefficients that were identified relative to adherence to condom use and weapon safety are available in Appendix 4.1, Significant Pearson Correlations for Condom Use and Weapon Safety.

Significant bivariate Pearson correlation coefficients: Malaria chemoprophylaxis medications. There were 31 significant bivariate Pearson correlation coefficients that were identified relative to the KAP and environmental domains and the malaria chemoprophylaxis outcome variables Take meds as directed and Intend to take meds the next time. These 
correlation coefficients revealed both positive and negative linear relationships and are

illustrated in Tables 4.1 and 4.2.

Table 4.I. Significant Bivariate Correlation Coefficients with Dependent Variable, Take Meds as Directed $(\mathrm{n}=150)$

Variable

1. Demographics, Age

2. Do not believe malaria meds are important in combat.

3. Believe others take meds as directed.

4. Believe others "do not" take as directed.

5. When in region, do you think of malaria regularly?

6. How often did you take the meds?

7. When reminded I take, otherwise often forget.

8. Plan malaria meds into missions.

9. I advised friends to take the meds as directed.

10. I advised friends to take the meds as directed. (Ever/Never)

11. I discussed the malaria threat with friends.

12. I discussed the malaria threat with friends. (Ever/Never)

13. I discussed decision "not" to take meds as directed. (Ever/Never) $\quad-.206^{*}$

14. How often did Command recommend discuss importance of meds? $.213^{\star}$

${ }^{*}$ Correlation is significant at the 0.05 level (2-tailed).

** Correlation is significant at the 0.01 level (2-tailed). 
Table 4.2. Significant Bivariate Correlation Coefficients with Dependent Variable, Intend to Take Meds Next Time $(\mathrm{n}=150)$

$\underline{\text { Variable }}$

Pearson Correlation Coefficient

1. Unit of Assignment

$-.221^{*}$

2. Knowledge (Gradable) - Which is not a malaria medicine.

$.231^{*}$

3. Knowledge (Gradable) - Can Military punish for not taking meds? $.295^{\star *}$

4. Friends think OK for me, multi-day mission, not take as directed. $\quad-.281^{\star \star}$

5. Friends think OK if I lie about meds.

$-.252^{* *}$

6. If I were a leader, friends think I should set example to take meds. $.287^{* *}$

7. How often did you take the meds? $.583^{* *}$

8. (Ever/Never): How often did you take the meds?

$.469^{* *}$

9. Plan malaria meds into missions.

$.532^{* *}$

10. (Ever/Never): Plan malaria meds into missions. $.567^{\star \star}$

11. I discussed decision "not" to take meds as directed.

12. I discussed decision "not" to take meds as directed. (Ever/Never) $-.392^{* *}$

13. I advised friends to take the meds as directed. $.326^{* \star}$

14. I advised friends to take the meds as directed. (Ever/Never) $.324^{* \star}$

15. I discussed the malaria threat with friends. (Ever/Never) $.227^{*}$

16. I had education on malaria meds and feel it prepared me well. $.292^{* \star}$

17. Education on meds is offered at current unit.

* Correlation is significant at the 0.05 level (2-tailed).

${ }^{* *}$ Correlation is significant at the 0.01 level (2-tailed).

The strongest correlation coefficient in Table 4.1 is the variable, How often did you take the meds? The strongest correlation coefficients in Table 4.2 were variables, How often did you take the meds, Plan malaria meds into missions, and the ever/never form of Plan malaria meds into missions. 
Rejecting the First Null Hypothesis. The results of these multiple bivariate Pearson correlation coefficients have identified multiple significant positive and negative linear relationships between the KAP domains, environmental domains, and the behavioral outcomes. Therefore, at an alpha of 0.05 , the first Null Hypothesis can be rejected.

\section{The Second Null Hypothesis}

In order to accept or reject the second Null Hypothesis, binary logistic regression models were used to determine if any predictive relationships that may exist between the independent variables and the outcomes (Faul et al. 2009; Fox, 2008; Kleinbaum, Kupper, Muller, \& Nizam, 1998). Any significant effects were based on an alpha of 0.05 . Odds ratios were interpreted to understand the potential predictive impact of the independent variables on the outcome. The $95 \%$ confidence intervals were also considered and interpreted to understand the range of effects the odds ratios may represent.

Multiple significant logistic regression relationships were identified for each of the three behaviors. Because the over arching future goal of this research is to improve adherence to malaria chemoprophylaxis medications, and given that the second null hypothesis considers behavioral intentions, only the significant relationships relative to the dependent variable Intend to take meds (malaria chemoprophylaxis medications) next time are reported in this chapter. All other significant findings will be provided upon request sent to the author at cmbtdvr@hotmail.com.

Combined models. The combined model is considered the most informative model because it combines all the significant variables from each of the eleven (11) domains into one combined model. Therefore, the combined model is utilized in this chapter to identify and describe any significant predictive relationships.

As outlined in the methods section, two types of combined models were used in this analysis. For review, the full combined model contains variables that include each of the 
responses or dummy variables that were accepted for analysis (e.g., 0-None of the time, 1Some of the time, 2-Most of the time, and 3-All of the time, etc.). The ever/never combined model contains only dichotomized ever or never dummy variables (e.g., 0-Never and 1-Ever). The results of each model are presented.

Full combined model. Employing the minimum 0.05 criterion of significance, the full version of the combined model includes two significant independent variables relative to the dependent variable Intend to take meds next time. The first significant variable was Plan malaria meds into missions and the second was I discussed decision "not" to take meds as directed.

The final model and results of the logistic regression analyses are presented in Table 4.3.

Table 4.3. Final Full Combined Logistic Regression Model, Predicting the Intention to Take the Medications the Next Time $(n=150)$

Variable $\quad \underline{B} \quad \underline{S E} \underline{\text { Wald } x^{2}} \underline{\rho \text {-value }} \underline{\underline{O d d s} \text { ratio }} \quad \underline{95 \% \mathrm{Cl}}$

1. Plan malaria meds into

missions.

$27.96 \quad 0.001$

$\mathrm{DV}^{\dagger}$ 1: Some of the time $4.59 \quad 1.73 \quad 7.05 \quad 0.008 \quad 98.13^{*} \quad[3.34,2880.09]$

DV 2: Most of the time $4.85 \quad 1.40 \quad 11.92 \quad 0.001 \quad 127.48^{*}[8.135,1997.84]$

DV 3: All of the time $\quad 4.72 \quad 1.44 \quad 10.66 \quad 0.001 \quad 111.64^{*}[6.597,889.18]$

2. I discussed decision

"not" to take meds

as directed.

$8.54 \quad 0.036$

$\mathrm{DV}^{\dagger}$ 1: Once

$-4.35 \quad 1.87$

$5.45 \quad 0.020$

$0.01 \quad[0.00,0.498]$

DV 2: A few times

$-3.36 \quad 1.29$

$6.89 \quad 0.009$

$0.03^{*} \quad[0.003,0.425]$

DV 3: Many times

$-1.74 \quad 1.81$

$0.92 \quad 0.337$

$0.18 \quad$ [0.005 to 6.09$]$

† Dummy Variable

* Odds Ratios may be inflated due to small cell sizes 
Table 4.3 shows that the variable Plan malaria meds into missions has significant main effects on the dependent variable Intend to take meds next time. The three corresponding dummy variables all have significant partial predictive effects. Controlling for the other variable in this model, for each unit increase in the dummy variable 1 (Some of the time), study participants who selected this variable may be 98.13 times more likely to increase their probability of a yes membership to the dependent variable. Additionally, with the same controls, for each unit increase in the dummy variable 2 (Most of the time), study participants who selected this variable may be 127.48 times more likely to increase their probability of a yes membership to the dependent variable. Finally, again controlling for the other variable, for each unit increase in dummy variable 3 (All of the time), study participants who selected this variable may be 111.64 times more likely to increase their probability of a yes membership to the dependent variable Intend to take meds next time. The $95 \%$ confidence intervals for each of these dummy variables are wide and may capture wide ranges of potential effects; nevertheless, they remain significant.

Table 4.3 also shows that the variable I discussed decision "not" to take meds as directed, has significant main effects on the dependent variable Intend to take meds next time. In this case, only dummy variable 2 (A few times) had significant predictive effects. Controlling for the other variable in this model, for each unit increase in this dummy variable, study participants who selected $A$ few times, may be $97 \%$ less likely to increase their probability of a yes membership to the dependent variable, Intend to take meds next time. The $95 \%$ confidence interval for this variable has a very reasonable width, indicating there are limited variations in potential effects.

Validation of Odds Ratios. The odds rations for the variable Plan malaria meds into missions illustrated in Table 4.3 are high and require some form of validation. This will be accomplished by reviewing the frequencies of each response noted in the cross tabs. The 
complete SPSS@ cross tabs output relative to this variable is available in Appendix 4.3. Nevertheless, the results of the cross tabs are also explained here in narrative format.

In the cross tab output, it is noted that the frequencies of responses to the reference variable (none of the time) was high at 49 of the 148 recorded responses, equating to $33.1 \%$ of the responses. Additionally the responses to dummy variables one, two, and three are relatively low. For example dummy variable one (some of the time) was selected 21 times, or $14 \%$ of the total recorded responses. Dummy variable two (most of the time) was selected 39 times, or $26.4 \%$ of the responses. Dummy variable three (all of the time) was selected 24 times or $16.2 \%$.

On the other hand, the outcome variable Intend to take meds the next time was responded to affirmatively with a yes response by a high percentage of the Ranger participants. The frequency of yes responses was 90 of the 148 recorded responses, equating to $60.8 \%$ of the responses. Given the high number or affirmative responses to this outcome variable, relative to the frequency of the reference variable (none of the time) was selected, it would be expected that these odds ratios would be high and that they would correspond reasonably to the response rates.

In other words, given these frequencies, the odds ratio 98.13 could be expected to correspond relatively to a $14 \%$ variable response rate. The odds ratio 127.48 might be expected to correspond with the $26.4 \%$ variable response rate. The odds ratio 111.64 might also be expected to correspond to the $16.2 \%$ variable response rate. As previously discussed, the $95 \%$ confidence intervals are wide with each of these variables, which can indicate a wide range of potential effects.

Despite what is presented within this validation, these inflated odds ratios may also be due to small cell sizes and could represent an example of bias due to sparse matched sets of data. This bias is not evident in the ever/never models. 
Ever/never combined model. Employing the minimum 0.05 criterion of significance, the ever/never version of the combined model includes three (3) significant independent variables relative to the dependent variable Intend to take meds next time. These three variables include Plan malaria meds into missions, I discussed decision "not" to take meds as directed, and I advised friends to take the meds as directed. Each of these three variables has significant predictive effects on the dependent variable. The final model and results of the logistic regression analyses are presented in Table 4.4.

Table 4.4. Final Ever/Never Combined Logistic Regression Model, Predicting the Intention to Take the Medications the Next Time $(n=150)$

Variable $\underline{\beta} \quad \underline{S E} \underline{\text { Wald } x^{2}} \quad \underline{\rho \text {-value }}$ Odds ratio $\quad \underline{95 \% \mathrm{Cl}}$

1. Plan malaria meds into missions.
$\mathrm{DV}^{\dagger}$ 1: Ever
$2.67 \quad 0.66$
$16.46<0.001$
14.41
$[3.97,52.23]$

2. I discussed decision "not"

to take meds as directed.
DV 1: Ever
$-2.16 \quad 0.68$
10.09
0.001
0.12
$[0.03,0.44]$

3. I advised friends to take

the meds as directed.
DV 1: Ever
$1.55 \quad 0.67$
5.40
0.020
4.72
$[1.27,17.48]$

† Dummy Variable

Controlling for the other variables in the Table 4.4 model, for each unit increase in the ever/never version of the variable Plan malaria meds into missions, study participants who selected this variable may be 14.41 times more likely to increase their probability of a yes membership to the dependent variable, Intend to take meds next time. Additionally, with the same controls, for each unit increase in the variable I discussed decision "not" to take meds as 
directed, study participants who selected this variable may be $88 \%$ less likely to increase their probability of a yes membership to the dependent variable, Intend to take meds next time. Finally, for each unit increase in the ever/never version of the variable I advised friends to take the meds as directed, study participants who selected this variable may be 4.72 times more likely to increase their probability of a yes membership to the dependent variable. The $95 \%$ confidence intervals for each of these three ever/never variables have reasonable widths; indicating that they have limited variations of possible effects.

Rejecting the Second Null Hypothesis. The results of these binary logistic regression models have identified multiple significant predictive relationships relative to the KAP and environmental domain variables. These findings indicate their potential ability to predict behavioral intentions, as a proxy for behavior change. Therefore, employing the minimum 0.05 criterion of significance, the second Null Hypothesis can be rejected.

\section{The Third Null Hypothesis}

In order to accept or reject the third Null Hypothesis, significant Command Support domain variables were identified through their interactions with variables in the domains of perceived susceptibility and risk, behavior outcome related practices, interpersonal communications, and the behavior outcomes. Any significant effects were based on an alpha of 0.05. This analysis included ten (10) moderator interaction models. These models identified two (2) moderator variables and four (4) significant interaction terms. The results of these analyses are described and presented in Table 4.5.

As previously mentioned, the over arching future goal of this research is to improve adherence to malaria chemoprophylaxis medications; therefore, only the significant results relative to malaria chemoprophylaxis medications are reported in this chapter. All other significant findings will be provided upon request sent to the author at cmbtdvr@hotmail.com. 


\section{Moderator interactions with the outcome variable, Intend to take meds the next}

time. Unfortunately, there were no significant moderator interactions relative the outcome variable Intend to take meds next time. Therefore, only those moderator variables and interaction term results relative to the outcome variable Take meds as directed are presented.

Moderator interactions with the outcome variable, Take meds as directed. There were four (4) significant moderator interactions identified relative to command support domain moderator variables and their interaction with variables in the domains of perceived susceptibility and risk, behavior outcome related practices, and interpersonal communications relative to the outcome variable Take meds as directed. In each of the four interactions, the significant Command Support moderator variable was How often does the Command include importance of taking meds in unit safety brief? 
Table 4.5. Moderator Variables and their Significant Interaction Terms, Relative to the Dependent Variable Take Meds as Directed $(n=150)$

1. Domain: Perceived Susceptibility and Risk

Moderator variable: How often does the Command include importance of taking meds in unit safety brief? $\left(\mathrm{DV}^{\dagger} 2 \text {, a few times) }\right)^{£}$

Significant Interaction term: When in region, do you think of malaria regularly? $\left(\mathrm{DV}^{\dagger} 1\right.$, yes) is moderated by How often does the Command include importance of taking meds in unit safety brief? $\left(\mathrm{DV}^{\dagger} 2 \text {, a few times) }\right)^{£}$
$\underline{\beta} \quad \underline{S E}$
$\frac{\text { Wald } x^{2}}{10.99}$
$\underline{\rho \text {-value }}$
Odds ratio
$95 \% \mathrm{Cl}$
2.29
0.69
$10.99 \quad 0.001$
9.87
$[2.55,38.22]$

2. Domain: Perceived Susceptibility and Risk

Moderator variable: How often does the Command include importance of taking meds in unit safety brief? $\left(\mathrm{DV}^{\dagger} 1 \text {, ever) }\right)^{\ddagger}$

Interaction term: When in region, do you think of malaria regularly? $\left(\mathrm{DV}^{\dagger} 1 \text {, yes) }\right)^{\mathfrak{1}}$ is moderated by the ever/never version of How often does the Command include importance of taking meds in unit safety brief? $\left(\mathrm{DV}^{\dagger} 1 \text {, ever }\right)^{\ddagger}$

$\begin{array}{llllll}\underline{\beta} & \underline{S E} & \frac{\text { Wald } x^{2}}{14.96} & \frac{\rho \text {-value }}{<0.001} & \underline{\text { Odds ratio }} & \frac{95 \% \mathrm{Cl}}{5.98}\end{array}$

3. Domain: Behavior Outcome Related Practices

Moderator variable: How often does the Command include importance of taking meds in unit safety brief? $\left(\mathrm{DV}^{\dagger} 1 \text {, ever) }\right)^{\ddagger}$

Interaction term: The ever/never version of Plan malaria meds into missions (DV ${ }^{\dagger} 1$, ever) ${ }^{\ddagger}$ is moderated by the ever/never version of How often does the Command include importance of taking meds in unit safety brief? $\left(\mathrm{DV}^{\dagger} 1 \text {, ever }\right)^{\ddagger}$
$\frac{\beta}{1.41} \quad \underline{S E}$
$\underline{\text { Wald } x^{2}}$
o-value
Odds Ratio
$95 \% \mathrm{Cl}$
12.70
4.08
$[1.88,8.85]$

4. Domain: Interpersonal Communications

Moderator variable: How often does the Command include importance of taking meds in unit safety brief? $\left(\mathrm{DV}^{\dagger} 1 \text {, ever) }\right)^{\ddagger}$.

Interaction term: The ever/never version of I advised friends to take the meds as directed. $\left(\mathrm{DV}^{\dagger} 1 \text {, ever }\right)^{\ddagger}$ is moderated by the ever/never version of How often does the Command include importance of taking meds in unit safety brief? $\left(\mathrm{DV}^{\dagger} 1\right.$, ever) ${ }^{\ddagger}$

$\begin{array}{llllll}\underline{\beta} & \frac{S E}{0.39} & \frac{\text { Wald } x^{2}}{15.29} & \frac{\rho \text {-value }}{<0.001} \quad \frac{\text { Odds Ratio }}{4.63} & \frac{95 \% \mathrm{Cl}}{[2.148,9.99]}\end{array}$

† Dummy Variable

$£$ Full Version

$\ddagger$ Ever/Never Version

Note: Significance values are for the Interaction Term, as calculated in SPSS $®$. 
Table 4.5 shows there are two (2) significant moderator interaction terms identified within the domain of Perceived Susceptibility and Risk, one (1) within the domain of Behavior Outcome Related Practices, and one (1) within the domain of Interpersonal Communications. The significant moderator variable in these interactions is How often does the Command include importance of taking meds in unit safety brief? This moderator variable was significant three (3) times in the ever/never form, and only once (1) in the full dummy variable form.

\section{Interpretations of the four (4) moderator interaction models.}

1. Domain: Perceived Susceptibility and Risk.

Moderator Variable: How often does the Command include importance of taking meds in unit safety brief?

Significant Interaction Term: The independent variable When in region, do you think of malaria regularly? is moderated by the Command Support variable How often does the Command include importance of taking meds in unit safety brief? Outcome variable: Take meds as directed.

At an alpha of 0.05 the interaction effect between the independent variable and the moderator variable has significant effects on the model relevant to the dependent variable Take meds as directed. Controlling for all other variables in this model, the moderator variable How often does the Command include importance of taking meds in unit safety brief? moderates the relationship between the variable When in region, do you think of malaria regularly? and the outcome variable Take meds as directed.

Considering this moderator interaction and when holding other variables constant; for each unit increase in the dummy variable 1 (yes) of the independent variable and dummy variable 2 (a few times) from the Command Support moderator variable, survey participants who selected these dummy variables may be 9.87 times more likely to increase their probability of a "yes" membership to the outcome variable Take meds as directed. The $95 \%$ confidence 
interval for this interaction is also reasonable, which indicates there are limited variations in the potential effects.

In other words, Ranger Command Support in the form of including the importance of taking malaria medications a few times in the unit safety briefing moderates the relationship between thinking about malaria on a regular basis, a few times, and Rangers taking their malaria chemoprophylaxis medications as directed. Therefore, the predictive potential for the Rangers to take their malaria chemoprophylaxis medications as directed is increased due to this moderated interaction.

This is a change from the non-moderated binary logistic regression model of the independent variable When in region, do you think of malaria regularly, relative to the same dependent variable. The non-moderated model yielded a significance of 0.001 and an odds ratio of 3.99. Given the same parameters, this moderated relationship has increased the potential for probability of a "yes" membership to the outcome variable (Take meds as directed) from 3.99 times more likely to 9.87 times more likely, an increase of $247 \%$.

2. Domain: Perceived Susceptibility and Risk.

Moderator Variable: How often does the Command include importance of taking meds in unit safety brief (ever/never version of variable)

Significant Interaction Term: The independent variable, When in region, do you think of malaria regularly? is moderated by the ever/never version of the Command Support variable How often does Command include importance of taking meds in unit safety brief?

Outcome variable: Take meds as directed.

At an alpha of 0.05 the interaction effect between the independent variable and the moderator variable has significant effects on the model relevant to the dependent variable Take meds as directed. Controlling was not significant in this model. 
Though the variables in this model may appear the same as those in interaction number 1 , the model is slightly different. The moderator variable in this model is in the ever/never version, while the significant independent variable remained the same as in the first interaction model. In other words, the ever/never version of the moderator variable, How often does the Command include importance of taking meds in unit safety brief also moderated the relationship between the variable When in region, do you think of malaria regularly, and the outcome variable Take meds as directed. This interaction had lesser effects than those seen in interaction number 1 ; nevertheless, the $95 \%$ confidence interval was slightly narrower.

For each unit increase in the dummy variable 1 (yes) of the independent variable and dummy variable 1 (ever) from the Command Support moderator variable, survey participants who selected these dummy variables may be 5.98 times more likely to increase their probability of a "yes" membership to the outcome variable Take meds as directed. The $95 \% \mathrm{Cl}$ for this interaction is reasonably narrow, which indicates there are limited variations in the potential effects.

In other words, Ranger Command Support in the form of ever including the importance of taking malaria medications in the unit safety briefing moderates the relationship between thinking about malaria on a regular basis, and Rangers taking their malaria chemoprophylaxis medications as directed. Therefore, the predictive potential for the Rangers to take their malaria chemoprophylaxis medications as directed is increased due to this moderated interaction.

This is again a change from the non-moderated binary logistic regression model of the independent variable When in region, do you think of malaria regularly, relative to the same dependent variable. Again, the non-moderated model yielded a significance of 0.001 and an odds ratio of 3.99. Given the same parameters, the moderated relationship has increased the potential for probability of a "yes" membership to the outcome variable (Take meds as directed) from 3.99 times more likely to 5.98 times more likely, an increase of $49.8 \%$. 


\section{Domain: Behavior Outcome Related Practices}

Moderator Variable: How often does the Command include importance of taking meds in unit safety brief (ever/never version of variable)

Significant Interaction Term: The ever/never version of the independent variable Plan malaria meds into missions is moderated by the ever/never version of the Command Support variable How often does the Command include importance of taking meds in unit safety brief?

Outcome variable: Take meds as directed.

At an alpha of 0.05 the interaction effect between the independent variable and the moderator variable has significant effects on the model relevant to the dependent variable Take meds as directed. Controlling was not significant in this model.

Both the moderator and independent variables in this model are in the ever/never versions. In other words, the ever/never version of the independent variable Plan malaria meds into missions is moderated by the ever/never version of the Command Support variable How often does the Command include importance of taking meds in unit safety brief?

For each unit increase in the dummy variable 1 (ever) of the independent variable and dummy variable 1 (ever) from the Command Support moderator variable, survey participants who selected these dummy variables may be 4.08 times more likely to increase their probability of a "yes" membership to the outcome variable Take meds as directed. The $95 \% \mathrm{Cl}$ for this interaction is reasonably narrow, which indicates there are limited variations in the potential effects.

In other words, Ranger Command Support in the form of ever including the importance of taking malaria medications in the unit safety briefing moderates the relationship between Rangers ever planning malaria meds into missions, and their taking their malaria chemoprophylaxis medications as directed. Therefore, the predictive potential for the Rangers 
to take their malaria chemoprophylaxis medications as directed is increased due to this moderated interaction.

This interaction is again a change from the non-moderated binary logistic regression model of the independent variable Plan malaria meds into missions, relative to the same dependent variable, but is rather counterintuitive.

Though the moderated interaction increases the predictive potential for the Rangers to take their malaria chemoprophylaxis medications, the moderated model yielded slightly lesser effects on the outcome than the non-moderated model.

The non-moderated model yielded a significance of 0.001 and an Odds Ratio of 4.96. Given the same parameters, the moderated model has decreased the potential for probability of a "yes" membership to the outcome variable (Take meds as directed) from 4.96 times more likely to 4.08 times more likely; a decrease of $17.7 \%$ from the non-moderated model.

4. Domain: Interpersonal Communications.

Moderator Variable: How often does the Command include importance of taking meds in unit safety brief (ever/never version of variable)

Significant Interaction Term: The ever/never version of the independent variable I advised friends to take the meds as directed, is moderated by the ever/never version of the Command Support variable How often does the Command include importance of taking meds in unit safety brief?

Outcome variable: Take meds as directed.

At an alpha of 0.05 the interaction effect between the independent variable and the moderator variable has significant effects on the model relevant to the dependent variable Take meds as directed. Controlling was not significant in this model. 
Both the moderator and independent variables in this model are in the ever/never versions. In other words, the ever/never version of the independent variable I advised friends to take the meds as directed, is moderated by the ever/never version of the Command Support $\mathrm{v}$

For each unit increase in the dummy variable 1 (ever) of the independent variable and dummy variable 1 (ever) from the Command Support moderator variable, survey participants who selected these dummy variables may be 4.63 times more likely to increase their probability of a "yes" membership to the outcome variable Take meds as directed. The $95 \% \mathrm{Cl}$ for this interaction is reasonably narrow, which indicates there are limited variations in the potential effects.

In other words, Ranger Command Support in the form of ever including the importance of taking malaria medications in the unit safety briefing moderates the relationship between Rangers ever advising their friends to take the malaria chemoprophylaxis medications as directed, and their taking their malaria chemoprophylaxis medications as directed. Therefore, the predictive potential for the Rangers to take their malaria chemoprophylaxis medications as directed is increased due to this moderated interaction.

Nevertheless, this interaction is again a change from the non-moderated binary logistic regression model of the independent variable I advised friends to take the meds as directed, relative to the same dependent variable, but again is rather counterintuitive.

Though the moderated interaction increases the predictive potential for the Rangers to take their malaria chemoprophylaxis medications, the moderated model yielded slightly lesser effects on the outcome than the non-moderated model.

The non-moderated model yielded a significance of 0.001 and an Odds Ratio of 5.54. Given the same parameters, the moderated model has decreased the potential for probability of a "yes" membership to the outcome variable (Take meds as directed) from 5.54 times more likely to 4.63 times more likely; a decrease of $16.4 \%$ from the non-moderated model. 
Results of the four (4) moderator interactions. The results of these moderated logistic regression models have identified multiple significant moderator interaction terms relative to the Command Support domain moderator variable How often does the Command include importance of taking meds in unit safety brief. This moderator variable has been shown to have significant effects on variables within the domains of perceived susceptibility and risk, behavior related practices, and interpersonal communications relative to the outcome variable Take meds as directed.

Rejecting the Third Null Hypothesis. These findings indicate that Command Support can moderate the domain variables included in the third Null Hypothesis. Therefore, at an alpha of 0.05 , the third Null Hypothesis can be rejected.

\section{Illustration of Knowledge Relative to the Behaviors}

This illustration was not part of any research question, but was thought to be informative to describe the Ranger population's knowledge relative to the three behaviors. It also gives an anecdotal perspective of how knowledge, given the questions that were included in the survey instrument, may reflect on the adherence levels of the dependent variables.

The analysis was performed on the gradable knowledge questions in the survey instrument. The questions were scored based on a $0-100 \%$ scale.

The cross tab calculations were run on each of the gradable knowledge variables and a grade was given to the entire Ranger population. Correct scores were based on selection of the most correct response for each variable. If $90 \%$ or greater of all participants from each Ranger unit selected the correct response, the Ranger population was given credit for responding correctly to that variable. One exception to this rule was given: If four of the five Ranger units $90 \%$ or above and only had an $80 \%$ or greater score, the population was given credit for a correct response. A list of the correct responses can be found in Appendix 4.2, Correct Survey Questionnaire Knowledge Responses. 
There were eleven (11) gradable variables for Malaria Chemoprophylaxis Medications, thirteen (13) gradable variables for Condom Use, and ten (10) gradable variables for Weapon Safety. Outside of this illustration, the knowledge variables were all selected for further analysis through the same methods described in Chapter III. These knowledge scores and their relation to the dependent variables will be further discussed in Chapter V.

\section{Gradable Knowledge Results}

Based on a scale ranging from $0-100 \%$, the knowledge scores for the Ranger population, relative to the three behaviors are as follows:

1. Based on the calculation method and the questions within the survey instrument, there were three (3) out of eleven (11) questions answered correctly by the Ranger population relative to malaria chemoprophylaxis medications. This represented a knowledge score for this behavior of $27.27 \%$.

2. Based on the calculation method and the questions within the survey instrument, there were seven (7) out of thirteen (13) questions answered correctly by the Ranger population relative to condom use. This represented a knowledge score for this behavior of $53.85 \%$.

3. Based on the calculation method and the questions within the survey instrument, there were six (6) out of ten (10) questions answered correctly by the Ranger population relative to weapon safety. This represented a knowledge score for this behavior of $60.0 \%$.

\section{One-Way Analysis of Variance}

One-way ANOVA analyses were conducted to examine differences between the responses given by the Ranger participants to two independent variables relative to the outcome variable Intend to take meds next time. The two independent variables were: Plan malaria meds into missions and I discussed decision "not" to take meds as directed. 
The One-way ANOVA analysis did not reveal any statistically significant differences among responses given by Rangers assigned to any of the five different units, relative to the independent variable Plan malaria meds into missions; F $(4,128)=2.039$, $\rho$-value $=0.085$, or relative to the independent variable I discussed decision "not" to take meds as directed; F (4, 140) $=1.579, \rho$-value $=0.183$

Post-hoc multiple comparisons testing through both Scheffe's and Bonferroni methods, conducted on both independent variables, did not reveal any statistically significant differences between the responses given from participants assigned to any of the five Ranger units. Details of this analysis are available upon request sent to the author at cmbtdvr@hotmail.com.

\section{Summary of Chapter Results}

In spite of not achieving the initial goal to recruit $4.3 \%$ of each unit population and minimal over sampling, each Ranger unit was well represented and the maximum sample size of 150 was achieved. Those participants who were recruited appear to closely resemble the demographic estimates of the Ranger population with respect to multiple demographic parameters including age, rank and ethnicity. In other words, the study sample demographics appear to be representative of the Ranger Regiment population.

The results of the bivariate Pearson correlations have identified multiple significant positive and negative linear relationships between the KAP domains, environmental domains, and the behavioral outcomes. Therefore, at an alpha of 0.05 , the first Null Hypothesis can be rejected

Results of the binary logistic regression models have identified multiple significant predictive relationships relative to the KAP and environmental domain variables. These findings indicate their potential ability to predict behavioral intentions, as a proxy for behavior change. Therefore, at an alpha of 0.05 , the second Null Hypothesis can be rejected. 
Results of the moderated logistic regression models have identified multiple significant moderator interaction terms relative to the Command Support domain moderator variable How often does the Command include importance of taking meds in unit safety brief. This moderator variable has been shown to have significant effects on variables within the domains of perceived susceptibility and risk, behavior related practices, and interpersonal communications relative to the outcome variable Take meds as directed. These findings indicate that Command Support can moderate the domain variables included in the third Null Hypothesis. Therefore, at an alpha of 0.05 , the third Null Hypothesis can be rejected.

Based on a scale ranging from $0-100 \%$, knowledge scores for the Ranger population were reported. Though these scores are anecdotal, they will be discussed in some detail in Chapter V.

A limited number of One-way ANOVA analyses with Post-hoc multiple comparisons testing were conducted. These analyses did not reveal any statistically significant differences among responses that were analyzed. This analysis suggests the responses are homogenous across the Ranger Regiment population. 


\section{CHAPTER V}

DISCUSSION

\section{Opening Statement}

Adherence to malaria chemoprophylaxis medications in U.S. military populations, including the Ranger population, is notoriously low. The reasons these personnel choose not to take these preventive medications properly are not clear. No studies exist that thoroughly examines the relevant KAPs associated with this behavior in the Ranger population. This exploratory study will help to fill this gap in the current knowledge and also inform future research and/or interventions aimed at improving adherence to malaria chemoprophylaxis medications in the Ranger population, as well as throughout the military. The U.S. Army Public Health Command is currently awaiting the completion of this study in order to inform and plan future research and/or interventions based on these results.

This study aimed to identify and understand the KAPs associated with three different health behaviors, each with varying levels of adherence (high, medium, low). The three health behaviors studied are 1) adherence to weapon safety guidance, 2) adherence to condom use guidance, and 3) adherence to malaria chemoprophylaxis medications. The main conceptual idea of this study was that if the KAPs affecting the more highly adhered to behaviors were identified and better understood, they then could be compared to those KAPs affecting the lesser adhered behaviors. This would, in concept, lead to a clearer understanding of the behavioral dynamics affecting these behaviors in this Ranger population and thereby improve conceptualization, development, operationalization, and implementation of future research and/or interventions within this population.

Unfortunately, this concept did not prove to be as productive and/or informative as initially conceptualized. The results from adherence to condom use and weapon safety did not prove to be beneficial to improving the understanding of the behavioral dynamics affecting 
adherence to malaria chemoprophylaxis medications. Additionally, the logistic analyses of the highly adhered behavior, weapon safety, were inconclusive. The adherence levels were so high to the weapon safety survey responses that the logistic regression models could not be improved; therefore, making comparisons based on these models between the highly adhered behavior and the lower adhered behaviors were not possible.

Therefore, because 1) the initial concept of comparing the three behaviors was not as effective as planned, 2) because the main focus of this research was to improve adherence to malaria chemoprophylaxis medications, and 3) given that the results relevant to adherence to malaria chemoprophylaxis medication alone were reported in Chapter IV; only those findings associated with adherence to malaria chemoprophylaxis medications will be discussed within this chapter. Two exceptions to this will be made. There will be a discussion relative to the reported adherence to each of the three behaviors measured in the survey instrument, as well as a discussion on the anecdotal knowledge findings. Other than these two exceptions, only the significant results relative to the Null Hypotheses and adherence to malaria chemoprophylaxis medications will be discussed.

\section{Discussion of Results}

\section{Description of the Participants}

Despite not being able to maintain the initial goal of equally sampling $4.3 \%$ of each Ranger; the slight over sampling of the RHQ and the RSTB, as well as the slight under sampling of the 3rd Ranger Battalion did not appear to impact the representativeness of the sample of participants that were recruited. Those who were recruited appear to closely resemble the initial demographic estimates of the Ranger population with respect to age, rank, and ethnicity; as noted in figures $4.1,4.2$, and 4.3. In other words, the study sample demographics appear to be representative of the Ranger Regiment population. 


\section{Levels of Adherence and Intentions to Adhere}

There were some slight discrepancies between the dependent variables that measured adherence to the three behaviors (e.g., Take Meds as Directed, Use a Condom, and Follow Weapon Safety) and some of the questions within the survey instrument which asked more detail about the behaviors. For example, the dependent variable questions simply asked, did you adhere to the behavior exactly as directed, yes or no? The more detailed questions that followed asked if the participant followed the behavior 1) none of the time, 2) $25 \%$ of the time, 3 ) $50-75 \%$ of the time, or 4) $100 \%$ of the time.

Nevertheless, in some cases there was a discrepancy in how those who answered "yes I did adhere as directed," also answered "25\% or $50 \%-75 \%$ of the time," in the more detailed questions. For example and concerning the entire study population of 150 participants, $8.0 \%$ of those who answered "yes, I did adhere to the malaria chemoprophylaxis medications as directed," also answered that they took them only " $25 \%$ or $50 \%-75 \%$ of the time." Similar discrepancies occurred with condom use (4.1\% discrepancy) and following weapon safety $4.0 \%$ discrepancy). I would like to attempt to account for what may have caused these discrepancies.

First, the participants could have potentially felt they might have at some point in their life 1) missed one or two doses of malaria chemoprophylaxis, 2) failed to use a condom, or 3) had some sort of lax in weapon safety at one or more times. Each of these adherence scenarios could have been only momentary or rare. The point is that these Rangers might have felt they could not have answered truthfully that they adhered $100 \%$ of the time, without exception.

In support of this I submit my more than 24 years of personal observation that the Rangers are a very honest group of soldiers with very high levels of integrity. It is possible that the more detailed and quantifying questions were simply too specific to be truthfully answer as $100 \%$ by these Rangers. The more general bivariate questions, with yes or no responses were 
easier to answer as being adherent. In all honesty, I think that it would be rare to find someone who would do something $100 \%$ of the time, without exception.

Secondly, another explanation may be that some of the Ranger participants truly believe that taking a medication 90 or $99 \%$ of the time is being adherent. This could be the same for the other behaviors. For example, during combat there are many instances where a lax in strict weapon safety may be necessary for any number of reasons. For this reason, some participants may believe that deviating from strict $100 \%$ adherence is necessary and legitimized at times and therefore, $100 \%$ adherence may even be impossible.

A third explanation may be that these differences in responses are simply mistakes, or even some form of amusement by the participants to confuse the researcher. Nevertheless, multiple conjectures could be proposed and discussed, but the reality is that these differences in responses did not occur with any great frequency. Therefore, I consider the overall self reported adherence levels to be reasonable representations of the behaviors within this population of Ranger participants.

Another finding concerning adherence to malaria chemoprophylaxis medications (Took as directed), which was reported by the Ranger participants and measured at $32.9 \%$, was that this was very similar to the findings reported in the literature by Kotwal et al. (2005). The reported by Kotwal et al. reported overall adherence to malaria chemoprophylaxis medications, in a Ranger population, to be $31 \%$. I find this to be a rather amazing similarity. Therefore, I believe it is reasonable to assume that adherence levels to malaria chemoprophylaxis medications in Ranger populations may generally range near $30 \%$.

The Ranger's Intentions to adhere the next time to malaria chemoprophylaxis medications was only reported at $60.8 \%$. There are no references in the literature to support or explain intentions to adhere to these medications, but I believe it is an important finding given the fact that the literature does support the $100 \%$ adherence requirement to maintain adequate 
serum protection for malaria. Perhaps the logistic regression findings of this study can help improve the intentions to adhere in this population. The odds ratios discussed relative to the second research question appear to predict that the independent variables Plan malaria meds into missions and I advised friends to take the meds as directed may significantly improve the outcomes relative to the intentions to take these medications next time.

As for condom, the reported level of adherence in the Ranger participants was $10.1 \%$. This is well below the $43 \%$ adherence reported by U.S. Army members who participated in the 2008 Department of Defense survey of health related behaviors among active duty military personnel. I believe this low level of adherence may have been due to the survey instrument, which may not have accurately accounted for the high number of married participants, which was reported at $51.7 \%(n=150)$ of the participants. There were also other concerns, such as extramarital or sexual relations that were not explored by the survey instrument. I believe more research and further validation of the survey instrument will be necessary to more accurately capture the adherence to condom use in this population.

The intentions to use a condom the next time were reported at $52.7 \%$ in this population, which is a potential improvement. Unfortunately, the logistic regression analyses failed to find any significant predictive relationships concerning condom use variables and intentions to use condoms next time outcome. Nevertheless, I believe more research is necessary and a refined, validated survey instrument will be required to accurately capture adherence to this behavior, in this population. If you will recall in the Methods chapter; this survey instrument was primarily designed around adherence the malaria chemoprophylaxis medications. The responses in condom use and weapon safety sections of the instrument were all closely base of these malaria chemoprophylaxis questions.

Concerning weapon safety, this was the first known study that measured adherence and/or intentions to adhere to weapon safety. Following weapon safety guidance has never 
been the subject of any known Department of Defense, federal law enforcement agency, or National Rifle Association study to date. A detailed search through multiple federal, state, and academic databases and search engines did not recover any weapon safety behavior related research. The data in the study is the first known sample, most certainly from any Special Operations group of soldiers.

The reported adherence level to following weapon safety (follow at all times) was $98.6 \%$. The intentions to follow the next time were somewhat lower at $97.3 \%$. I cannot explain why the intentions to follow next time are slightly less than the actual reported adherence levels to weapon safety. Though there is only a $1.3 \%$ difference, this is appears counter intuitive. Perhaps it is a function of some inaccuracy that the survey instrument is measuring and should be further examined in the final validation process. As discussed above, the survey instrument was primarily designed around adherence to malaria chemoprophylaxis medications. Though there may be some inaccuracies, the adherence levels are high and indicate this behavior is most likely followed often in this population of Rangers.

The high levels of adherence relative to weapon safety was not conducive to logistic regression analyses because there was little variance in any logistic regression model. The high adherence created logistic regression models that had little room for improvement.

The three theories supporting this study; the Health Belief Model (HBM), the Theory of Reasoned Action/Theory of Planned Behavior (TRA/TPB), and the Social Cognitive Theory (SCT) can also explain these levels of adherence through the fundamental concepts of (a) perceived severity, benefits, barriers, and susceptibility; (b) behavioral intentions, attitudes towards behavioral outcomes, power, and control; and (c) self efficacy, vicarious learning, reinforcement, reciprocal determinism; and the interaction between the individual, the environment, and the behavior. This is illustrated in the theoretical diagram presented in Figure 7 of Chapter III. 
As described in Chapter II, adherence to medical therapies, including malaria chemoprophylaxis medications involves multiple and complex phenomena. Each of the concepts taken from the three supporting theories named above can either individually or in combination affect these levels of adherence. Take for example, perceived severity: considering this concept and the adherence identified here associated with following weapon safety, it appears that the Rangers who participated in this study perceive the severity associated with this behavior high. Given the void in literature concerning this behavior and considering the intuitive impact one would expect risk perception to have on weapon safety: I believe it can be assumed that risk perception may be a major factor relative to this behavior.

The impact of risk perception on adherence to medical therapies, including malaria chemoprophylaxis medications is referenced numerous times in the literature. For example, a 2008 study by AstraZeneca of 200 physicians produced a list of the top-10 factors that affect adherence to medical therapies (Koroneos, 2008): low perceived personal risk was number five on that list of factors. Additionally, low perceived risk was reported by Ben-Ami et al. (2005), Garges (2007), as well as LaFon and Scoville (2006) as ) as factors that negatively impact the decision to adhere to malaria chemoprophylaxis medications.

\section{The Research Questions}

This study focused on answering three research questions. Each research question was answered and the corresponding Null Hypothesis then rejected. As previously noted, this study focused primarily on adherence to malaria chemoprophylaxis medications; therefore, the significant findings relative to this behavior discussed here.

\section{Research Question 1}

The first research question states, "What relationship, if any, exists between the current KAP domains, environmental domains, and behavioral outcomes?" The Null Hypothesis 
supporting this research question is, "No relationship exists between the current KAP domains, environmental domains, and behavioral outcomes."

Through the analysis process and employing the 0.05 criterion of statistical significance, multiple linear relationships were identified. This effectively answered this first research question. The results bivariate correlations do not imply causation and each must be interpreted independently. Some interpretations are very straight forward, while others are not. For example: the variable M18L stated, "Can the military punish you for not taking your malaria chemoprophylaxis medications as recommended?" This variable was found to have a significant positive Pearson correlation coefficient of 0.295 ( $\rho$-value 0.01$)$. One could interpret this to signify that punishment may positively influence adherence. In fact, it is only the knowledge that the military can punish which is significant in this correlation. It is not the punishment per se that is associated with this relationship. Nevertheless, the overall findings of this research show there are significant relationships relative to the KAP and environmental domains that which were included in the survey instrument.

There were 31 significant bivariate Pearson correlation coefficients identified relative to adherence to malaria chemoprophylaxis medications. Each of these is illustrated in Tables 4.1 and 4.2. Discussing the details of each of these coefficients is not within the limits of this dissertation; therefore, I will only discuss the strongest coefficients for each outcome.

The strongest correlation coefficient in Table 4.1, relative to the outcome Take meds as directed, is the variable How often did you take the meds, which had a positive Pearson correlation coefficient of 0.716 . The interpretation of this coefficient is relatively straight forward. The more the participants took their medications, the more likely they were to take them as directed. This is somewhat intuitive, but this is also supported by the findings of Sherbourne et al. (1992) who studied past histories of adherence in nearly 2000 hypertension, diabetes, and heart disease patient. The study found that the past history of adherence was the strongest 
predictor of future adherence, particularly non-adherence. Therefore, given this high coefficient, it might be expected that the more a Ranger took his malaria medications, the more likely the chance that they would be taken as directed.

The strongest correlation coefficients in Table 4.2, relative to the outcome Intend to take meds the next time, are 1) How often did you take the meds (correlation coefficient 0.583), Plan malaria meds into missions (correlation coefficient 0.532), and 2) the ever/never form of the same variable Plan malaria meds into missions (correlation coefficient 0.567). Similarly, the interpretations of these coefficients are also relatively straight forward. The more often the participants take their medications and plan them into their missions, the more likely the participants to intend to take their medications the next time. This again appears somewhat intuitive to me, but is again substantiated by the findings of others in the literature.

The findings of Sherbourne et al. (1992), as discussed above concerning the frequency of taking the medications, remain the same for this outcome. Concerning the next variable, Plan malaria meds into missions, I explain this much differently. There is a void in the literature concerning planning medications into military missions; nevertheless, there are similar behaviors and findings noted in the literature. For example, the act of planning a medication into military missions would imply that the medications are taken more seriously as a priority and that there would be a basic understanding of the treatment regimen. Multiple literary articles including Amico (2009), Osterberg and Blaschke (2005), and DiMatteo et al. (1993) discuss the importance of making the medications a priority and have a better understanding of the treatment regimen to maintain adherence.

Additionally, planning the medications into military missions could impact forgetfulness. In other words, it could aid the participant's memory to take the medications. Forgetfulness is well noted in the literature as having a negative impact on adherence (Amico, 2009; Bosworth et al., 2005; Kravitz et al., 1993, WHO, 2003). In fact, forgetfulness has been particularly noted in 
the few malaria chemoprophylaxis studies that focused on military populations (Garges, 2007; LaFon, \& Scoville, 2006).

There again 31 significant Pearson correlation coefficients. The coefficients discussed here are just the beginning, but it appears that if the discussions could be expanded to include each and every significant coefficient, I believe either the literature or any of the three theories supporting this study could help explain them.

\section{Research Question 2}

The second research question states, "Can any of the behavioral domain variables predict behavioral intentions, as a proxy for behavior change?" The Null Hypothesis supporting this research question states, "The current KAP domain variables cannot predict behavioral intentions, as a proxy for behavior change."

Utilizing binary logistic regressions and employing the 0.05 criterion of statistical significance, this analysis produced two models that may predict behavioral intentions as a proxy for behavior change. This effectively answered the second research question.

Because the focus of this research question considered behavioral intentions, only the significant predictive models relative to the dependent variable Intend to take meds next time will be discussed. These models are presented as combined models, as discussed in the methods. The full combined model will be discussed first, followed by the ever/never combined model.

The full combined model. There are two significant independent variables identified within the full combined model 1) Plan malaria meds into mission, which had positive predictive impacts on the outcome, and 2) I discussed decision "not" to take meds as directed, which had negative predictive impacts on the outcome. This model is illustrated in Table 4.3.

The first independent variable, Plan malaria meds into missions contained three dummy variables, each with significant odds ratios. These odds ratios are large, but may represent huge 
potential positive predictive impacts. In other words, if a Rangers plans malaria medications into their missions Some of the time, the potential to intend to take malaria chemoprophylaxis medications as directed the next time may be increased to 98.13 times more likely. Additionally, if they plan them into missions Most of the time, the potential may be increased to 127.48 times more likely. Finally, if they plan them into missions All of the time, the potential may be increased to 111.64 times more likely. Though these odds ratios are very high, I have validated them by response frequencies noted in the cross tabs. This is explained in some detail in the results chapter.

The $95 \%$ confidence intervals for each of these variables are also wide and represent a wide range of effects (e.g., [3.34, 2880.09] for Some of the time). Nevertheless, I believe the odds rations are significant and may represent the potential to produce rather larger predictive impacts within this population relative to the intention to take malaria chemoprophylaxis medications the next time.

The second independent variable in the model, I discussed decision "not" to take meds as directed, had only one significant dummy variable. This variable, $A$ few times, produced a significant odds ratio of 0.03 . This represents huge potential negative predictive impact on the outcome. In other words, if Rangers discusses the decision "not" to take malaria chemoprophylaxis medications as directed only $A$ few times, their potential to intend to take them as directed the next time may be decrease by $97 \%$. The $95 \%$ confidence interval for this variable was also relatively wide (e.g., $[0.003,0.425]$ for DV2) and represent a wide range of effects. Nevertheless, I believe this odds ratio is significant and may represent the potential to produce rather larger predictive impacts within this population relative to the intention to take malaria chemoprophylaxis medications the next time.

The ever/never combined model. There were three significant independent variables identified within the ever/never combined model: 1) Plan malaria meds into mission, which has a 
positive predictive impact on the outcome; 2) I discussed decision "not" to take meds as directed, which again has a negative predictive impact on the outcome, and 3) I advised friends "to take" the meds as directed, which has a positive predictive impact on the outcome. These are all dichotomous variables and the odds ratios are much more reasonable than those in the full combined model. The results are illustrated in Table 4.4.

The first independent variable, Plan malaria meds into mission (ever/never) produced an odds ratio of 14.41 . This again represents a rather large potential positive predictive impact on the outcome. In other words, if Rangers ever plan malaria chemoprophylaxis medications into their missions, the potential to intend to take these medications as directed the next time may be increased 14.41 times more likely. The $95 \%$ confidence interval for this variable is also somewhat wide (i.e., $[3.97,52.23]$ ) and may indicate a wide range of effects on the outcome.

The second independent variable, I discussed decision "not" to take meds as directed produced an odds ratio of 0.12 . This represents a rather large potential negative predictive impact on the outcome. In other words, if Rangers ever discuss the decision "not" to take the malaria chemoprophylaxis medications as directed, the potential to intend to take these medications as directed the next may be decreased by $88 \%$. The $95 \%$ confidence interval for this variable is again wide (i.e., $[0.03,0.44]$ ), and may also indicate a wide range of effects on the outcome.

The third independent variable, I discussed decision "to take" meds as directed, produced an odds ratio of 4.72 . This is again a substantial potential positive predictive impact on the outcome. In other words, if Rangers ever discuss the decision "to take" malaria chemoprophylaxis medications as directed, the potential to intend to take these medications as directed the next time may be increased by 4.72 times more likely. The $95 \%$ confidence interval for this variable is relatively narrow (i.e., $[1.27,17.48]$ ), indicating a relatively narrow range of effects on the outcome Intend to take meds next time. 
Theoretical explanations for the combined model results. To explain the affect planning medications into missions may have on the outcome Intend to take meds next time, I believe components of both the Health Belief Model (HBM) and the Theory of Reasoned Action/Theory of Planned Behavior (TRA/TPB) are well suited.

The HBM can help explain this positive impact planning may have by a) improving the Ranger perceived severity of malaria, b) the planning process may improve awareness of the benefits to taking the medications, c) planning will improve barriers by making the medications more readily available to the Rangers, and d) the planning process itself may increase the Ranger's awareness of his susceptibility malaria.

Components of the TRA/TPB also help to explain this outcome, particularly through a) the impact planning may have on improving behavioral intentions through purposeful actions designed to mold future behavior (e.g., planning to have the medications taken at certain times and locations), b) the positive impact planning may have on the Ranger attitudes towards the behavioral outcome of taking the medications and thereby avoiding potential malaria infections, and c) the positive impacts that the planning process may have on the perceived power and control the Rangers have over preventing malaria infections by taking these medications.

To explain the negative impact that discussing the decision "not" to take meds as directed may have on the outcome Intend to take meds next time, I believe components of the Social Cognitive Theory (SCT) are well suited in this case given the components of self efficacy, vicarious learning, and reinforcement. For example, it is rather intuitive to expect that negative interpersonal communications focused on not taking these medications would have a negative effect on self efficacy. It is also seems obvious that vicarious learning would be negatively affected by such conversations. Negative interpersonal communications may also reinforce any potential intentions not to take these medications that a Ranger may already be considering. Finally, as the SCT explains, these negatively focused interpersonal communications may result 
in an interaction between the individuals involved in the discussions, their environment, and the behavior outcome; which could help explain the negative predictive impact these interpersonal communications may have.

Alternatively, the SCT can also be used to explain the positive impact that discussing the decision "to take" meds as directed may have on the outcome Intend to take meds next time. Given the same example as mentioned above, it is again rather intuitive to expect that positive interpersonal communications focused on taking these medications would have a positive effect on self efficacy. It also seems obvious that vicarious learning would be positively affected by such conversations. Positive interpersonal communications may also reinforce any potential intentions to take these medications that a Ranger may already be considering. Finally, as the SCT again explains, these positively focused interpersonal communications may result in an interaction between the individuals involved in the discussions, their environment, and the behavior outcome; which could help explain the positive predictive impact these interpersonal communications may have.

References within the literature. The findings in the relevant literature concerning the independent variable Plan malaria meds into missions are presented in some detail above with the first research question. The variables I discussed decision "not" to take meds as directed and I discussed decision "to take" meds as directed, are also referenced similarly in the literature. In other words, it is my belief that the same references reported above concerning planning malaria medications into missions, particularly those references that discuss the importance of placing a priority on taking these medications and also those that discuss forgetfulness, can be transposed on to the latter two variables concerning interpersonal communications. For example, it is my belief and it appears again rather intuitive, that both affirmative and positive interpersonal communications can impact the importance and priorities placed on taking these medications. Additionally, these same communications may impact 
forgetfulness. As a review, the importance of placing a priority on taking medications is referenced multiple articles (Amico, 2009; DiMatteo et al., 1993; Osterberg \& Blaschke, 2005), as is forgetfulness (Amico, 2009; Bosworth et al., 2005; Kravitz et al., 1993; WHO, 2003). Remember that forgetfulness was also referenced in the few malaria chemoprophylaxis studies that focused on military populations (Garges, 2007; LaFon, \& Scoville, 2006).

Aside from these references that tie the themes relative to planning and interpersonal communications together; interpersonal communications may also be linked to social support, which has been referenced by multiple authors concerning adherence to medical therapies. DiMatteo (2004), Martin et al. (2005), the World Health Organization (2003), the RAND Corporation (2009), as well as others all place a high level of importance on social support to maintaining adherence to medical therapies.

Effective communication is noted as an important predictor, factor, and/or determinate of adherence to medical therapies in multiple references (Bosworth et al., 2005; DiMatteo et al., 1993; NCPIE, 2007; Osterberg \& Blaschke, 2005; WHO, 2003), but I make this link between interpersonal communications and social support because of a recurring theme that will resurface in the third research question, where the importance of social support will again be discussed.

\section{Research Question 3}

The third research question states, "Do variables within the environmental domains of behavioral education, behavior related training, behavior related media, or military command support moderate the relationship between any KAP domain variables?" The Null Hypothesis supporting this research question states, "Command Support domain variables do not moderate the relationship between variables within the KAP domains of perceived susceptibility and risk, behavior related practices, interpersonal communications, and the behavior outcomes." 
Utilizing the moderated logistic regression and employing the 0.05 criterion of statistical significance, this analysis produced four (4) significant moderator interactions relative to command support and the domains of perceived susceptibility and risk, behavior outcome related practices, and interpersonal communications relative to the outcome variable Take meds as directed. This effectively answered the third research question. The results are presented in Table 4.5.

There were no significant moderator interactions relative the outcome variable Intend to take meds next time. Therefore, only the significant moderator interactions relative to the dependent variable Take meds as directed will be discussed.

In each of the four interactions, the significant Command Support moderator variable was, How often does the Command include importance of taking meds in unit safety brief? This moderator variable was significant three (3) times in the ever/never form, and only once (1) in the full dummy variable form.

Two (2) significant moderator interaction terms were identified within the domain of Perceived Susceptibility and Risk, one (1) within the domain of Behavior Outcome Related Practices, and one (1) within the domain of Interpersonal Communications.

Despite the identification of a Command Support moderator variable and significant interaction terms between and the selected independent variables, each of these must be interpreted individually. Some of the interaction effects increased odds ratios while others decreased them. Each of these detailed interpretations of can be reviewed in Chapter IV; nevertheless, I will discuss the general impact each of the interaction.

The first moderator interaction is between the yes response from the dichotomous the independent variable When in region, do you think of malaria regularly, and the dummy variable, a few times, from the Command Support moderator variable How often does the Command 
include importance of taking meds in unit safety brief. This interaction produced a significant odds ratio of 9.87 .

The non-moderated odds ratio for the yes response in the variable When in region, do you think of malaria regularly was only 3.99. Therefore, this moderated relationship has increased the potential for a probability of a "yes" membership to the outcome variable (Take meds as directed) from 3.99 times more likely to 9.87 times more likely. This is an increase of $247 \%$. Additionally, the $95 \%$ confidence interval for this interaction is somewhat high with a moderate range of variations in the potential effects.

In short, this Command Support moderator interaction has shown the potential to increase the predictive potential for the Rangers to take their malaria chemoprophylaxis medications as directed by $247 \%$, compared to the non-moderated independent variable odds ratio.

The second moderator interaction is between the same yes response of independent variable When in region, do you think of malaria regularly, and the ever response from the ever/never version of the Command Support moderator variable How often does the Command include importance of taking meds in unit safety brief. This interaction produced an odds ratio of 5.89 .

Again, the non-moderated odds ratio for the yes response of variable When in region, do you think of malaria regularly was only 3.99. Therefore, this moderated relationship has increased the potential for a probability of a "yes" membership to the outcome variable (Take meds as directed) from 3.99 times more likely to 5.89 times more likely. This is an increase of $49.8 \%$, which is somewhat less than the first moderator interaction, but it remains an improvement from the non-interaction model. Additionally, the $95 \%$ confidence interval for this interaction is much narrower, which indicates there are limited variations in the potential effects. 
In short, this Command Support moderator interaction has increased the predictive potential for the Rangers to take their malaria chemoprophylaxis medications as directed by $49.8 \%$, compared to the non-moderated odds ratio.

The third interaction contained two ever/never variables. This interaction is between the ever response of the independent variable Plan malaria meds into missions, and ever response of the Command Support moderator variable How often does the Command include importance of taking meds in unit safety brief. This interaction produced an odds ratio of 4.08 .

In this case, the non-moderated ever response of the variable Plan malaria meds into missions produced a higher odds ratio of 4.96 . This is somewhat counter intuitive, because the impact of Command Support on planning would appear to have a positive rather than negative impact on the model. Nevertheless, this interaction has decrease the potential for a probability of a "yes" membership to the outcome variable (Take meds as directed) from 4.96 times more likely to 4.08 times more likely, a decrease of $17.7 \%$. The $95 \%$ confidence interval for this interaction is also very reasonable, which indicates there are limited variations in the potential effects.

In short and though it appears to be counter intuitive, the Command Support moderator interaction has slightly decreased the predictive potential for the Rangers to take their malaria chemoprophylaxis medications as directed by $17.7 \%$, compared to the non-moderated odds ratio.

The fourth interaction also contained two ever/never variables. This interaction is between the ever response of the independent variable I advised friends to take the meds as directed, and the ever response of the Command Support moderator variable How often does the Command include importance of taking meds in unit safety brief. This interaction produced an odds ratio of 4.63 . 
Again, the non-moderated model again produced a higher odds ratio. The nonmoderated ever response of the variable I advised friends to take the meds as directed produced and odds ratio of 5.54 vs. 4.63 . This is again appears to be counter intuitive because the impact of Command Support interpersonal communications, particularly affirmative communications, would appear to have a positive rather than negative impact on the model. Nevertheless, this moderated relationship has decreased the potential for a probability of a "yes" membership to the outcome variable from 5.54 times more likely to 4.63 times more likely, a decrease of $16.4 \%$. The $95 \%$ confidence interval for this interaction is also very reasonable, which indicates there are limited variations in the potential effects.

In short and though it again appears to be counter intuitive, but the Command Support moderator interaction has slightly decreased the predictive potential for the Rangers to take their malaria chemoprophylaxis medications as directed by $16.4 \%$, compared to the nonmoderated odds ratio.

Theoretical explanations for the moderated interactions. The theoretical support can also be used to explain the reasons Command Support has these moderator interaction effects on variables with the domains of perceived susceptibility and risk, behavior related practices, and interpersonal communications. First will refer to the Social Cognitive Theory (SCT). The SCT, in my view, always been the most beneficial theory for this research, particularly explaining the interaction of individuals within their immediate environment and the principal of operant conditioning; where individuals learn behaviors through positive and negative reinforcements (DiClemente, Crosby, \& Kegler, 2009; Edberg, 2007). Reinforcement is represented by Command Support and the commanders including the importance of taking malaria chemoprophylaxis medications in the unit safety briefs.

As discussed in Chapter III, the components of the SCT are divided into six individual characteristics and four environmental factors. The six individual characteristics include: self 
efficacy, behavioral capacity (knowledge and skill), expectations (outcomes), expectancies (good outcomes or rewards), self control (control over change), and emotional coping (ability to deal with change). The four environmental factors include: vicarious learning (through observing consequences), situation (perception of the environment), reinforcement (positive or negative responses to behavior), and reciprocal determinism (individual factors/social cues==>receive response==>adjust behavior, and so on). The Command Support of ...including the importance of taking meds in unit safety brief, could positively impact any one of these components either individually or in combination; perhaps it could impact all of them. The most obvious to me is the positive reinforcement it can have on influencing a behavior.

When a military Commander stands in front of his troops and give his or her unit safety brief (usually a weekly brief), it is a powerful positive reinforcement that can influence to continue with a desired behavior, or to intend to improve an undesirable behavior. I have both observed this behavior and personally experienced its effect on me repeatedly throughout my more than 24 years of military service.

References within the literature. The moderator variable How often does the Command include importance of taking meds in unit safety brief can not only be interpreted as a form of reinforcement, but in military populations this is also a form of social support. To explain this social support I will refer to my more than 24 years of personal observations as a soldier of how the unit or command and the soldiers in that command support each other.

Military members, particularly Rangers and other special operations members, consider the unit they are assigned to as an important social support system. The unit provides the level of support that is similar to what a close family environment may provide. Commanders often view their soldiers, particularly younger soldiers and particularly Rangers, as extensions of their own family. The opposite of this is also true where soldiers, again particularly Rangers, often consider their unit and/or commander as family. Through this relationship, they give each other 
emotional support on a number of levels and concerning many different types of behaviors. Emotional support is noted in multiple references including DiMatteo (2004), Martin et al. (2005), the World Health Organization (2003), the RAND Corporation (2009) as having significant impact on adherence to medical therapies.

The unit or command, as well as the soldiers within that command, also support each other in a practical sense as discussed by DiMatteo (2004); which includes instrumental support, assistance, reminders, and organizational support for behaviors. Both practical and emotional support are important variables in a meta-analysis (DiMatteo, 2004) examining the effects of multiple types of social support on adherence to various medical therapies.

To sum this up, the act of a commander mentioning any behavior in unit safety briefing has multiple impacts on the soldiers listening to these briefings. By giving these briefings, the commander who represents the command, is both reinforcing the behavior that his discussed (e.g., taking meds as directed), as well as providing social support on multiple levels. Each of these may impact the behavior taking malaria chemoprophylaxis medications in multiple aspects; either directly or by proxy through the impact the briefing may have on the members of the audience. I do not believe the true limits of this impact can or will ever be fully quantified, but identifying Command Support as a potential moderator variable in this study may be a good start.

\section{Illustration of Knowledge Relative to Adherence Levels}

The results of the gradable knowledge variables were not significant in any binary logistic regression equations or moderated regression analysis equations. Nevertheless, they do give an anecdotal illustration of the level of knowledge the Ranger participants may have relative to the questions I chose to include in the survey questionnaire.

As previously discussed, though the knowledge scores are anecdotal, it appears that the levels of knowledge did correspond with the levels of adherence found in the literature and that 
were predicted during the study conceptualization and proposal phases. For example:

knowledge scores to malaria chemoprophylaxis are lowest, then condom use is second or medium, and finally the knowledge scores relative to weapon safety are the highest. The literature revealed similar adherence levels to the three behaviors.

Unfortunately, the knowledge scores did not closely resemble the adherence levels that were attained by this research. In fact, the level of adherence to condom use was very different than the gradable knowledge scores found in this illustration (i.e., knowledge grade for condoms $27.27 \%$, level of adherence $10.1 \%$ ). The levels of adherence noted relative to weapon safety were also very different than the gradable knowledge scores that were identified (i.e., knowledge grade for weapons $60.0 \%$, adherence level $98.6 \%$ ). The only scores that were fairly similar were those relative to malaria chemoprophylaxis medications (i.e., knowledge grade for malaria medications $27.27 \%$, level of adherence $32.9 \%$ ).

Though this is an anecdotal illustration of graded knowledge, the low adherence reported by the Ranger participants to condom use $(10.1 \%)$ is not logical considering the graded knowledge score was $53.8 \%$. As previously mentioned, this adherence level does not at all resemble the level of adherence reported by U.S. Army members in the 2008 Department of Defense health related behavior survey which reported $43 \%$ adherence to condom use.

Perhaps the survey questions need to be reexamined to account for the potential of not applicable responses, though these were removed and the $10.1 \%$ adherence level was calculated only from those who answered yes or no to using a condom during sexual intercourse.

Perhaps this is a true level of adherence to condom use with respect to those Rangers who participated. I did observe multiple side-bar conversations between the Rangers who were either waiting to begin the survey or those who had just completed it, that were regarding a low perceived susceptibility and severity towards the risks of contracting any sexually transmitted 
disease. They appeared to think that no matter what, they could always just go to the military doctors and get treated. In this case the Health Belief Model would explain this behavior. Nevertheless, I cannot state whether or not this is the general consensus of the majority of Rangers who volunteered for this study.

It must be noted that the Ranger's graded knowledge with respect to weapon safety was surprising low at only $60 \%$. I found this surprising, considering this is a special operations forces population. It must also be noted that the questions chosen to measure their level of knowledge were somewhat obscure and not always relevant to the normal day-to-day operations of these Rangers. This being said, they were also no more obscure than the other questions supporting condom use or malaria medications.

By obscure, I am referring to questions that are not reflex related, commonsense-like, or those which are not regularly used their every day operations or conversations. An example of a more obscure question would be, "How many clicks to left do you move your front sight post when zeroing your weapon?" These are the questions that I thought measured true knowledge, not just reflex. I must also confess that often these type questions are not always known by all military members. They tend to have to study for these questions when a test is anticipated.

It was difficult to choose which questions to use to measure weapon safety knowledge. Most knowledge concerning weapon safety is simple, reflexive, and dictated by the immediate situation. For example, a common weapon safety question may be, "Is it safe to fire a weapon when a friendly human is in your line-of-sight?" Including questions such as this did not seem appropriate, because I felt the answers appeared to be clearly obvious and only measured common sense, rather than measuring true knowledge. Therefore, and particularly for this Ranger population, I decided to use the more obscure questions that came directly from the basic soldier skills manual. 
Given the graded weapon safety knowledge score of $60.0 \%$ score, it is clear that the Rangers, at least these participants, need to study the basic skills manual more frequently. But, I believe the Ranger's true knowledge of weapon safety is much higher than this illustration has revealed and that knowledge has yet to be captured accurately by this survey instrument. Perhaps there are other means of measuring this knowledge, which could be the focus of future studies.

In defense of all the all knowledge questions, this survey instrument was primarily designed to measure adherence to malaria chemoprophylaxis medications. The condom use and weapon safety questions only closely mirrored those base questions that more accurately measured adherence to malaria chemoprophylaxis medications.

Finally, though these illustrations of graded knowledge are anecdotal and do not lead to any significant analytic conclusions; this is the first time any of this data has been collected in this population. Given this fact, I felt these data are worth including in the results and discussion of this dissertation. They may someday become the cornerstone of future studies in this, as well as other military populations.

\section{Potential Homogeneity of the Population}

Though it was not a full analysis of each response given in the survey instrument, the limited number of ANOVA analyses and Post-hoc multiple comparisons testing conducted here did not reveal any statistically significant differences among responses given by Rangers assigned to any of the five different units. This suggests that the responses captured by the survey instrument are potentially homogenous across the Ranger Regiment.

\section{Conclusions}

This was an exploratory study within a population that is rarely granted access for behavioral research purposes. It was a complex study that captured an enormous amount of data which will contribute to the current base of knowledge. 
The study was conceived, implemented, and completed at a rapid pace; requiring detailed coordination and collaborations with multiple individuals from multiple organizations. This coordination and collaboration produced an original survey instrument which has shown the potential to capture significant findings. The maximum sample size was achieved and the data recovered from the participants has the potential to yield a variety of results. The study was well justified by the author and other military collaborators. It was accomplished well under the anticipated budget, and it appears to have produced actionable findings.

The demographics of the Rangers who volunteered to participate in this study indicate that the study captured a relatively representative sample of the 75th Ranger Regiment population. The discussions within this dissertation describing the homogeneity of the Ranger Regiment, as well as the limited ANOVA calculations, indicate that the survey responses are relatively homogenous across this population.

Therefore, I believe the results of this study can be reasonably compared to, if not generalized to, the overall 75th Ranger Regiment population and potentially other populations that share similar traits as the Ranger Regiment.

The overall self-report adherence levels to the three behaviors were not surprising, with exception to condom use, which was lower than expected and will undoubtedly lead for further studies to better understand this finding. The self-reported adherence to malaria chemoprophylaxis medications was similar to those reported Kotwal et al. (2005), and indicates a continued issue with non-adherence to these medications within this population of Rangers. Adherence to weapon safety was high, as expected, and represents a first time finding that may have multiple future implications.

The graded knowledge variables may or may not have accurately captured the true knowledge of the Ranger participants and furthermore, none of these variables were statistically significant predictors of behavioral intentions in any logistic regression analysis. Nevertheless, 
the illustrations of graded knowledge indicate that there may be a need to improve the general knowledge among the Ranger population relative to each of the three health behaviors.

The notion that was initially considered during the conceptualization of this study; that the findings relative to the higher adhered to behavior (in this case initially referring to Weapon Safety) could be transposed to the lesser adhered behavior (in this case referring to Malaria Chemoprophylaxis medications) in order to improve that behavior, have proved not to be a valid option in this case. There were simply not enough significant predictive findings relative to condom use or weapon safety that could be transposed on to those relative to adherence to malaria chemoprophylaxis medications. I have considered three potential reasons for this.

First, the survey instrument was primarily designed to measure KAPs relative to malaria chemoprophylaxis medications and not weapon safety or condom use. The Weapon Safety and Condom Use variables were only closely based as similarly as possible to the malaria variables. The condom and weapons questions were not as sophisticatedly designed and classically selected based on the previous interviews and focus group, as were the malaria questions. This could have contributed to the lack of significant findings relative to the weapon and condom behaviors.

Secondly, over $25 \%$ of the population of Rangers selected the not applicable (NA) option on the survey responses concerning adherence to condom use, indicating that they were in a long-term and strictly monogamous relationship. These participants then maintained this option throughout the survey. Therefore, this may have contributed to the lack of significant findings relative to this behavior.

Third, the overall self-reported adherence to Weapon Safety was $98.6 \%$. The majority of remaining responses chosen in the survey instrument affirmative and supported this high level of adherence. In other words, the $98 \%$ adherence was maintained throughout the survey by essentially every variable. This left very little variance to nearly all the weapon safety responses. 
Additionally, the high level of adherence gave no room for variables to improve any predictive models. This most likely contributed to the lack of significant logistic regression findings relative to this behavior.

Concerning the research questions and Null Hypotheses: I believe 1) each of the three research questions has been sufficiently answered and 2) the corresponding Null Hypotheses have all been sufficiently rejected.

The results of the multiple bivariate Pearson correlations identified multiple significant positive and negative linear relationships between the KAP domains, environmental domains, and the behavioral outcomes. The strongest correlation coefficients relative to each behavior have been discussed in some detail, but each of the 31 significant coefficients will require an individual interpretation. Though none of the Pearson coefficients imply can imply causation, the linear relationships have been identified.

The results of the binary logistic regression models identified multiple significant predictive impacts relative to the KAP and environmental domain variables. These findings indicate their ability to predict behavioral intentions, as a proxy for behavior change, particularly concerning 1) planning malaria chemoprophylaxis medications into military missions, and 2) maintaining affirmative interpersonal communications relative to taking these medications as directed.

The results of the moderated logistic regression models indicate that Command Support variables can moderate an interaction between variables within the domains of perceived susceptibility and risk; behavior related practices, interpersonal communications, and the outcome take meds as directed. The act of including the importance of taking malaria chemoprophylaxis medications in the unit safety briefings may have multiple implications as a moderator for adherence to these medications. Command support for this behavior has been 
discussed as being both a potential reinforcement, as well as a potential significant form of social support.

In summation, I believe the findings identified relative to the logistic regression combined models demonstrate the potential predictive impacts that 1) mission planning, and 2) and affirmative interpersonal communications may have concerning their potential to improve intentions to adhere to malaria chemoprophylaxis medications the next time. Also, the potential moderator interactions identified relative to Command Support may be indicate that including the importance of talking malaria chemoprophylaxis medications in unit safety briefings may be beneficial to improving adherence to these medications.

\section{Limitations}

This was a cross sectional study, implemented with an unvalidated anonymous survey instrument administered to a convenience sample of 150 volunteers assigned to a very specific organization within the Special Operations Command: the 75th Ranger Regiment. This presents an array of limitations.

The cross sectional study design is has generally two limitations (Kelsey, Whittemore, Evans \& Thompson, 1996):

1. The cross sectional study data lacks the power to imply cause and effect. Therefore, the results cannot imply causation.

2. The second limitation of cross sectional studies; that they may report a higher proportion of chronic disease. This was recognized as a limitation, but not considered relevant to this study because it did not study any chronic disease process.

The unvalidated survey instrument was a potential limitation to this study. The exploratory nature of this study, coupled with the time restraints regarding access to the Ranger population did not allow for the full validation of this instrument prior to administering it. Though 
unvalidated, the questionnaire did yield a number of significant findings sufficient to answer each of my research questions. A number of strengths, weaknesses, flaws, and areas that need improvement within this instrument have been identified and discussed in this dissertation; including the potential bias due to small cell sizes that may have inflated the odds ratios in Table 4.3. These will all be addressed prior to any future use of this instrument and will improve the final validation of this survey instrument.

This sample size was only 150 participants and the participant selection was of a convenience sample of volunteers. This was not a randomized sample of a large population; therefore, there no true external validity of these results. In defense of this, the gold standard of randomized selection and large sample sizes (Kelsey et al., 1996; Shadish, Cook, \& Campbell, 2002) was not a valid option in this study, and again, this study is not implying causation. As previously discussed, access to the Ranger population during their high operation tempo training and deployment cycles only afforded one reasonable option--to recruit a convenience sample. Additionally, the total population of the 75th Ranger Regiment is only 3500 personnel: recruiting 150 was an admirable task. In final defense, the power analyses conducted in support of this study indicated that the sample of 150 gave sufficient power to the chosen analytic techniques.

The Rangers of the 75th Ranger Regiment who participated in this study are very special individuals. Relative to the general Army population; Rangers are of above average intelligence, in excellent physical condition, highly motivated, highly responsive, and specially selected individuals. The Ranger Regiment is also a very special, high operations tempo, rapid deployment organization. As discussed in the Methods, these Rangers are not easily compared to any other populations, both within or outside the military. Therefore, even though generalizability is a recognized strength of cross sectional designed studies (Kelsey et al, 1996), it may be additionally difficult to generalize the findings of a study such as this, particularly of a 
special population, to other populations that do not share similar characteristics as found in these Rangers.

\section{Future Research}

This was an original study of a population to which researcher are rarely granted access. The 65-page survey instrument recovered an enormous amount of data. I believe the findings are actionable. Therefore, the findings of this research may open the door to many other areas of research. The findings may also be used to inform future interventions designed to improve the health of our military.

The significant logistic regression findings have identified the importance of mission planning and interpersonal communications in this population of Rangers. They also give a focused perspective for planning future interventions aimed at improving adherence to malaria chemoprophylaxis medications in this population, relative to the intention to take the medications the next time. For example, given the results of this study, if an intervention focused solely on improving planning malaria medications into Ranger missions it is possible that this could impact future adherence to these medications by an odds ratio of 14.41 . Other interventions focused on improving interpersonal communications could have similar results.

The significant logistic regression and moderator interaction findings are already being considered by the U.S. Army Public Health Command to aid in the conceptualization of future studies and interventions aimed at improving adherence to malaria chemoprophylaxis medications in the Ranger Regiment and other Special Operations units across the military. These findings may also similarly aid civilian researchers and public health professionals to plan future interventions or research aimed at better understanding these behaviors.

The KAPs and environmental domains relative to Weapon Safety have never been studied before. This data will be further studied an analyzed in order to promote and improve weapon safety in both military and civilian populations. The findings will be shared with the 
United States Army Combat Readiness/Safety Center, military commanders, and potentially with civilian law enforcement agencies to inform and support future research and/or interventions designed to better understand and/or improve to weapon safety.

The KAPs and environmental domains relative to Condom Use will also be further studied and analyzed to better understand why the self-report adherence to this behavior is surprisingly low relative to other Army populations surveyed in the Department of Defense survey of health related behaviors among active duty military personnel.

The review of the literature has shown that that improving adherence to medical therapies, including malaria chemoprophylaxis medications may require complex intervention techniques that will most likely utilize and maximize a combination of strategies to indentify poor adherence, simplifying therapies, reinforce desirable behaviors, improve communication and provider-patient relations, utilize technologies, improve support networks, educate stakeholders, and enlist the all involved into active participation. It is my hope that the findings of this study will be a helpful addition to the resources currently available to researchers and public health professionals who have this goal in mind and are focused on improving adherence to medical therapies such as malaria chemoprophylaxis medications. 


\section{LIST OF REFERENCES}

Abraham, C., Clift, S., \& Grabowski, P. (1999). Cognitive predictors of adherence to malaria prophylaxis regimens on return from malarious regions: A prospective study. Social Science and Medicine, 40(11), 1641-54. Retrieved from EBSCOhost Database.

Al-Adhroey, A., Nor, M., Al-Mekhlafi, H., \& Mahmud, R. (2010). Opportunities and obstacles to the elimination of malaria from Peninsular Malaysia: knowledge, attitudes and practices on malaria among aboriginal and rural communities. Malaria Journal 2010, 9(137). doi: 10.1186/1475-2875-9-137

Albert, N., Yancy, C., Liang, L., Zhao, X., Hernandez, A., Peterson, E.,...Fonarow, G. (2009). Use of Aldosterone antagonists in Heart Failure. JAMA, 302(15), 1658-65. Retrieved from the FIU CINAL Plus database.

Amico, K., Konkle-Parker, D.J., Cornman, D., Barta, W., Ferrer, R., Norton, W.,...Fisher, W. (2007). Reasons for ART non-adherence in the Deep South: Adherence needs of a sample of HIV-positive patients in Mississippi. AIDS Care, 19(10), 1210-18. Retrieved from the FIU CINAL Plus database.

Anastario, M. (2010). Barbados Survey Codebook. Document retrieved via email correspondence with Dr. Michael Anastario, Ph.D. Copy available upon request from cmbtdvr@hotmail.com.

Ayala, S., \& Spain, J. (1976). A population of Plasmodium Colombiense Sp. N. in the Iguanid lizard, Anolis Auratus. The Journal of Parasitology, 62(2), 177-89. Retrieved from the JSTOR Database at http://www.jstor.org/pss/3279261

Bangsberg, D., Moss, A., \& Deeks, S. (2004). Paradoxes of adherence and drug resistance to HIV antiretroviral therapy. Journal of Antimicrobial Chemotherapy, 53(5), 696-99.

Retrieved from PubMed database.

Barrier. (2012). Merriam-Webster Online Dictionary. Retrieved May 22, 2012 from http://www.merriam-webster.com/medical/barrier

Baudon, D., \& Martet, G. (1997). Malaria and Travelers: Protection and Information. Medicine Tropicale, 57(4), 497-500. Retrieved from the FIU Ovid Database.

Ben-Ami, R., Seigman-Igra, Y., Anis, E., Brook, G., Pitlik, S., Dan, M., \& Giladi, M. (2005). Malaria in travelers returning from short organized tours to holiday resorts in Mombasa, Kenya. The Israel Medical Association Journal, 7(6), 364-7. Retrieved from the CSA Illumina Database.

Berg, J., \& Visser, L. (2007). Expatriate Chemoprophylaxis Use and Compliance: Past, Present and Future From and Occupational Health Perspective. Travel Medicine, 14, 356-358. Retrieved from the FIU PubMed Database.

Bonkovsky, H., Tice, A., Yapp, R., Bodenheimer, H., Monto, A., Rossi, S., et al. (2008). Efficacy and Safety of Peginterferon Alfa-2a/ribavirin in Methadone Maintenance Patients: 
Randomized Comparison of Direct Observed Therapy and Self-Administration. American Journal of Gastroenterology, Nov; 1003(11), 2757-65. Retrieved from the FIU PubMed Database.

Bosworth, H., Olsen, M., \& Oddone, E. (2005). Improving blood pressure control by tailored feedback to patients and clinicians. The American Heart Journal, 149, 795-803.

Retrieved from http://pt.wkhealth.com/pt/re/merck/pdfhandler.00000406-20050500000008.pdf;jsessionid=L6yTDGzSwpGTNP7T2SDyyKQLfLHpZVR4ICf1719L7j84dx4XHG z2!215724456!181195629!8091!-1

Bradburn, N., Sudman, S., \& Associates. (1979). Improving Interview Method and Questionnaire Design. San Francisco: Jossey-Bass.

Breman, J.., Mills, A., Snow, R., Mulligan, J., Lengeler, C., Mendis, K.,...Doumbo, O. (Eds.). (2006). Conquering Malaria. Disease Control Priorities in Developing Countries (2nd Edition), 413-432. New York: Oxford University Press. Retrieved from http://files.dcp2.org/pdf/DCP/DCP21.pdf

Bronner, U., Divis, P., Farnert, A., \& Singh, B. (2009). Swedish traveler with Plasmodium knowlesi malaria after visiting Malaysian Borneo. Malaria Journal, 8(15), doi: 10.1186/1475-2875-8-15. Retrieved from http://www.malariajournal.com/content/8/1/15

Centers for Disease Control and Prevention. (2009a, May). Travelers' Health - Yellow Book, Chapter 5, Yellow Fever Vaccine Requirements and Information on Malaria Risk and Prophylaxis by Country. Retrieved May 31, 2009, from http://wwwn.cdc.gov/travel/yellowbook/ch5/malaria-yellow-fever-table.aspx

Centers for Disease Control and Prevention (CDC). (2009b, July). Travelers' Health-Yellow Book, Chapter 2, Pre- and Post-travel General Health Recommendations. Protection against Mosquitoes, Ticks, Fleas and Other Insects and Arthropods. Retrieved June 01, 2010, from http://wwwn.cdc.gov/travel/yellowbook/2008/ch2/insects-arthropods.aspx

Ciminera, P., \& Brundage, J. (2007). Malaria in US military forces: A description of deployment Exposures from 2003 through 2005. The American Journal of Tropical Medicine and Hygiene, 76(2), 275-79. Retrieved from the journal website at http://www.ajtmh.org/cgi/reprint/76/2/275

Cleemput, I., Kesteloot, K., \& DeGeest, S. (2002). A review of the literature on the economics of noncompliance. Room for methodological improvement. Health Policy, 59(1), 65-94. Retrieved from the Science Direct Journals database.

Cockburn, A. (1963). The evolution and eradication of infectious diseases. Baltimore: The Johns Hopkins Press.

Compliance alliance: Identify problems, but work with team. (2010, April). Clinical Trials Administrator, 8(4), 37-39. Retrieved from the FIU CINAL Plus database. 
Congressional Research Service (CRS). (2007). CRS Report for Congress. Military airlift:

C-17 aircraft program. Retrieved from http://www.fas.org/sgp/crs/weapons/RL30685.pdf

Converse, J.M., \& Presser, S. (1986). Survey Questions: Handcrafting the Standardized Questionnaire. Beverly Hills, CA: Sage Publications.

Cook, G., \& Zumla, A. (Eds.). (2008). Manson's Tropical Disease, 22nd Edition. Retrieved from the FIU MD Consult database.

Dasgupta, B. (1965). Blood parasites in the Himalayan flying squirrel. Transactions of the Royal Society of Tropical Medicine and Hygiene. 59(6), 716-17. Retrieved from Science Direct database.

Department of Defense (DoD). (2008). 2008 Department of Defense survey of health related behaviors among active duty military personnel. Retrieved from http://www.tricare.mil/2008HealthBehaviors.pdf

Department of State (DoS). (2005). Medical evacuation. U.S. Department of State Foreign Affairs Manual, 7(364.5), 17. Retrieved from http://www.state.gov/documents/organization/86599.pdf

Department of the Army (DA). U.S Army Regulation 40-46. (2009). Clinical Quality Management. Washington, DC: US Army Publishing Department. Retrieved June 03, 2010 from http://www.army.mil/usapa/epubs/pdf/r40_68.pdf

Department of the Army (DA). U.S Army Regulation 40-3. (2010). Medical, Dental, and Veterinary Care. Washington, DC: US Army Publishing Department. Retrieved June 03, 2010 from http://www.army.mil/usapa/epubs/pdf/r40_3.pdf

DeShano, R. (2011). Walter Reed Army Institute of Research, Data Coordinating and Analysis Center Survey Booklet Production Costs. Retrieved via email correspondence with Mr. Rory DeShano on March 02, 2011. Copy available upon request from Mr. Michael Pagel at cmbtdvr@hotmail.com.

Determinant. (2012). Merriam-Webster Online Dictionary. Retrieved May 22, 2012 from http://www.merriam-webster.com/dictionary/determinant

DiClemente, R.J., Crosby, R.A., \& Kegler, M.C., (Eds.). (2009). Emerging Theories in Health Promotion, Practice, and Research. San Francisco: Jossey-Bass.

DiMatteo, M., Hays, R., \& Sherbourne, C. (1992). Adherence to cancer regimens: Implications for treating the older patient. Oncology, 6(2 Suppl.). Abstract received June 05, 2010 from the FIU PubMed database. Full article reprint received July 10, 2010, from The RAND Reprint Series, Santa Monica, CA: RAND.

DiMatteo, M., Sherbourne, C., Hays, R., Ordway, L., Kravitz, R., McGlynn, E.,...Rogers, W. (1993). Physicians' characteristics influence patients' adherence to medical treatment: Results from the Medical Outcomes Study. Health Psychology, 12(2), 93-103. Retrieved from http://psycnet.apa.org.ezproxy.fiu.edu/journals/hea/12/2/93.pdf 
DiMatteo, M. (2004). Social support and patient adherence to medical treatment: A metaanalysis. Health Psychology, 23(2), 207-18. Retrieved May 24, 2010 from http://www.unl.edu/HAVLAB/Attachments/Adherence\%20Articles/Dimatteo\%202004.pdf

Don't get complacent with hand hygiene. (2010, March). ED Nursing, 54-55. Retrieved from the FIU CINAL Plus database.

Dorland's Pocket Medical Dictionary (25th ed.). (1995). Philadelphia, PA.: W.B. Saunders Company.

Dupouy-Camet, J., Yera, H., \& Tourte-Schaeffer, C. (2003). Problems in Prescribing Malaria Chemoprophylaxis for Travelers. Fundamental \& Clinical Pharmacology, 17(2), 161-9. Retrieved from the FIU PubMed Database.

Edberg, M. (2007). Essentials of Health Behavior, Social and Behavioral Theory in Public Health. Boston: Jones and Bartlett Publishers.

Epocrates on-line. (May 30, 2009). Malaria Infection-Primary Prevention. Retrieved May 30, 2009, from https://online.epocrates.com/noFrame/showPage.do?method= diseases\&Monographld=161\&ActiveSectionld=44

Factor. (2012). Merriam-Webster Online Dictionary. Retrieved May 22, 2012 from http://www.merriam-webster.com/dictionary/factor

Faul, F., Erdfelder, E., Buchner, A., \& Lang, A. (2009). Statistical power analyses using G*Power 3.1: Tests for correlation and regression analyses. Behavior Research Methods, 41, 1149-1160. doi:10.3758/BRM.41.4.1149. G*Power 3.1.2 downloadable from http://www.psycho.uni-duesseldorf.de/abteilungen/aap/gpower3/download-andregister

Ferketich, S. (1991). Focus on psychometrics: Aspects of item analysis. Research in Nursing \& Health, 14, 165-168. Retrieved from the FIU CINAL Plus database.

Forbes, V.J., (2010). Unawareness as a barrier to treatment $n$ patients with schizophrenia-A conceptual analysis. Journal of Psychosocial Nursing, 48(3), 30-36. Retrieved from the FIU CINAL Plus database.

Fox, J. (2008). Applied Regression Analysis and Generalized Linear Models (2nd ed.). Thousand Oaks, California: Sage Publications.

Garges, E., (2007). Malaria Prevention and Doxycycline Tolerability in the US Army $10^{\text {th }}$ Mountain Division: Preliminary Data Analysis . Walter Reed Army Institute of Research Power Point presentation. Presentation retrieved via email correspondence with Dr. Stephanie Scoville. Copy available upon request from cmbtdvr@hotmail.com.

General Accounting Office (GAO). (2000). Report to Congressional Requests, August 2000. Presidential travel, DoD airlift cost for White House foreign travel. Retrieved from http://www.gao.gov/archive/2000/ns00209.pdf 
General Services Administration (GSA). (2010). Uniformed service personnel travel. Joint Federal Travel Regulations, Volume 1, Change 278. Retrieved October 01, 2010 from http://www.defensetravel.dod.mil/pdc-archive/regchgs/monthly/2010/JFTR/Change\%20284\%20(08-01-10).pdf

GlaxoSmithKline LLC. (2010). Malarone (atovaquone and proquanil hydrochloride) tablet FDA Official Human Prescription Drug Label. Retrieved from http://dailymed.nlm.nih.gov/dailymed/druglnfo.cfm?id=19028\&CFID=47855487\&CFTOK $\mathrm{EN}=\mathrm{ab5}$ cf93654e9b0ce-0695DF3B-E9DA-290FD0E3A2698ECE875D\&jsessionid=ca30f2f23de253524d33

Going pro: Research monitoring program keeps sites in compliance. (2010). IRB Advisor, 10(4), 27-38. Retrieved from the FIU CINAL Plus database.

Golin, C., Liu, H., Hays, R.D., Miller, L., Beck, C.K., Ickovics, J.,...Wegner, N. (2002). A prospective study of predictors of adherence to combination antiretroviral medication. Journal of General Internal Medicine, 17, 756-65. Retrieved from the NIH database at http://www.ncbi.nlm.nih.gov/pmc/articles/PMC1495120/pdf/jgi_11214.pdf

Gostin, L.O. (2003). Module 1: Tradition, Profession, and Values in Public Health. In B. Jennings, J. Kahn, A. Mastroianni, \& L.S. Parker. (Eds.). Ethics and Public Health: Model Curriculum (pp. 13-36). Retrieved from http://www.asph.org/document.cfm?page $=782$

Grebely, J., Raffa, J., Meagher, C., Duncan, F., Genoway, K., Khara, M., et al. (2007). Directly Observed Therapy for the Treatment of Hepatitis C Virus Infection in Current and Former Injection Drug Users. Journal of Gastroenterol Hepatol, Sept; 22(9), 1519-25. Retrieved from the FIU PubMed Database.

Hagan, J., Marcos, L., \& Steinberg, T. (2010). Fever in a soldier returned from Afghanistan. Journal of Travel Medicine, 17(5), 351-52. Retrieved from the FIU Pub Med database.

Hays, R., Kravitz, R., Mazel, R., Sherbourne, C., DiMatteo, M., Rogers, H., \& Greenfield, S. (1994). The impact of patient adherence on health outcomes for patients with chronic disease in the Medical Outcomes Study. Journal of Behavioral Medicine, 17(4), 347-60. Retrieved June 07, 2010 from the FIU ILLiad Interlibrary Loan program. Abstract available at http://www.springerlink.com/content/q2367454x04p35h6/

He, N., Zhang, J., Yao, J., Tian, X., Zhao, G., \& Jiang, Q. (2010). Knowledge, attitudes, and practices of voluntary HIV counseling and testing among rural migrants in Shanghai, China. AIDS Education and Prevention, 21(6), 570-581.

doi:10.1521/aeap.2009.21.6.570

Hess, S. (2010). Conditional parameter estimates from Mixed Logit model: Distributional assumptions and a free software tool. Journal of Choice Modeling, 3(2), 134-52.

Retrieved March 4, 2012 from http://www.jocm.org.uk/index.php/JOCM/article/view/112/60 
Hewitt, R., (1941). Bird malaria. The American Journal of the Medical Sciences, 202(2), 264. Retrieved from The American Journal of the Medical Sciences website at http://journals.Iww.com/amjmedsci/Citation/1941/08000/Bird_Malaria.17.aspx.

Houston, D.J., \& Tuck, J.J. (2005). Malaria on a Military peacekeeping operation: A case study with no cases. Military Medicine, Mar; 170(3), 193-5. Retrieved July 2, 2009, from the FIU PubMed Database.

Hymel, P., \& Yang, W., (2008). Review of malaria risk and prevention for use in corporate travel. Journal of Occupational \& Environmental Medicine, 50(8), 951-9. Retrieved from the FIU CINAL Plus database.

Institution's reviews proactive, educational, and boost compliance. (2010, March). Clinical Trials Administrator, 34-33. Retrieved from the FIU CINAL Plus database.

Kalichman, S. (2008). Co-occurrence of treatment nonadherence and continued HIV transmission risk behaviors: Implications for positive prevention interventions. Psychosomatic Medicine, 70, 593-97. Retrieved from http://www.psychosomaticmedicine.org/cgi/reprint/70/5/593

Kalichman, S., Ramachandran, B., \& Catz, S. (1999). Adherence to Combination Antiretroviral Therapies in HIV Patients of Low Health Literacy. Journal of General Internal Medicine, 14(5), 267-73. Retrieved from http://pt.wkhealth.com/pt/re/merck/fulltext.00005125199905000-00001.htm;jsessionid=L7kTdQsjpN1Ks0y7LxSD7DQ7zTTFs OmvsIW0tpx4Q82PvvySfGHh!215724456!181195629!8091!-1?nav=reference

Keating, J., Eisele, T.P., Bennett, A., Johnson, D., \& Macintyre, K. (2008). A description of malaria-related knowledge, perceptions, and practices in the Artibonite Valley of Haiti: Implications for malaria control. The American Journal of Tropical Medicine and Hygiene, 78(2), 262-69. Retrieved from the FIU CINAL Plus database.

Kelsey, J., Whittemore, A., Evans, A., \& Thompson, W. (1996). Methods in Observational Epidemiology (2nd edition). New York: Oxford University Press.

Kheir, N., Greer, W., Yousif, A., Al-Geed, H., Al Okkah, R., \& Zirie, M. (2010). The utility of an electronic adherence assessment device in type 2 diabetes mellitus: a pilot study of single medication. Patient Preference and Adherence Journal 2010, 4, 247-254. Retrieved from the FIU PubMed database.

Kimura, M., Kawakami, K., Hashimoto, M., \& Hamada, M. (2006). Malaria prevention and stand-by emergency treatment among Japanese travelers. Travel Medicine Infectious Disease, 4(2), 81-85. Retrieved May 28, 2009, from the FIU PubMed Database.

Kitchener, S. J., Nasveld, P. E., Gregory, R. M., \& Edstein, M. D., (2005). Mefloquine and doxycycline malaria prophylaxis in Australian soldiers in East Timor. Medical Journal of Australia, Feb 21; 182(4): 168-71. Retrieved from the FIU PubMed Database.

Klein, D., Wustrack, G., \& Schwartz, A. (2006). Medication adherence: Many conditions, a common problem. Proceedings of the Human Factors and Ergonomics Society 50th 
Annual Meeting-2006. Retrieved from

http://www.ideo.com/images/uploads/thinking/publications/pdfs/HFES_IDEO-

adherence_2006.pdf

Kleinbaum, D., Kupper, L., Muller, K., \& Nizam. (1998). Applied Regression Analysis and Other Multivariate Methods (3rd ed.). Pacific Grove, California: Duxbury Press.

Koroneos, G. (2008, October 15). AstraZeneca digs into the cause of non-adherence. PharmExec.com. Retrieved from http://license.icopyright.net/user/viewFreeUse.act?fuid=ODQxODgyMg\%3D\%3D

Kotwal, R. S., Wenzel, R. B., Sterling, R. A., Porter, W. D., Jordan, N. N., \& Petruccelli, B. P. (2005). An outbreak of malaria in US Army Rangers returning from Afghanistan. The Journal of the American Medical Association (JAMA), 293(2), 212-16. Retrieved from the FIU CINAL Plus database.

Kravitz, R., Hays, R., Sherbourne, C., DiMatteo, M., Rogers, W, Ordway, L., \& Greenfield, S. (1993). Recall of recommendations and adherence to advice among patients with chronic medical conditions: Results from the Medical Outcomes Study. Archives of Internal Medicine, 153(16), 1869-78. Abstract retrieved from the FIU PubMed database.

Lack of adherence in heart failure therapy. (2010, April). Healthcare Benchmarks and Quality Improvement, 46-47. Retrieved from the FIU CINAL Plus database.

LaFon, S., \& Scoville, S. (2006). An outbreak of Plasmodium vivax malaria among US soldiers returning from Afghanistan. US Army Center for Health Promotion and Preventive Medicine Power Point presentation. Copy retrievable from www.pdhealth.mil/downloads/Outbreak.PPT

Landry, P., lorillo, D., Darioli, R., Burnier, M., \& Genton, B. (2006). Do travelers really take their mefloquine malaria chemoprophylaxis? Estimation of adherence by an electronic pillbox. Journal of Travel Medicine, 13(1), 8-14. Retrieved from the FIU CINAL Plus database.

Machtinger, E., \& Bangsberg, D. (2005). Adherence to HIV Antiretroviral Therapy . In: Peiperl, L., Coffey, S., Volberding, P., (Eds.). HIV InSite Knowledge Base [textbook online]; San Francisco: UCSF Center for HIV Information. Retrieved July 12, 2010 from http://hivinsite.ucsf.edu/InSite?page=kb-03-02-09

Martin, L., Williams, S., Haskard, K., \& DiMatteo, M. (2005). The challenge of patient adherence. Therapeutics and Clinical Risk Management, 1(3), 189-99. Retrieved from the NIH database at http://www.ncbi.nlm.nih.gov/pmc/articles/PMC1661624/pdf/tcrm0103-189.pdf

McCarthy, W.F., Guo, N. (2009). The Existence of Maximum Likelihood Estimates for the Logistic Regression Model. Paper SP02-2009, Maryland Medical Research Institute. Retrieved March 01, 2012 from http://www.pharmasug.org/download/papers/SP02.pdf

McPhee, S. J., Papadakis, M. A., \& Tierney, L. M. (Eds.). (2007). Current medical diagnosis \& treatment. New York: McGraw Hill. 
Munoz, M., Finnegan, K., Zeldita, J., Caldas, A., Sanchez, E., Callacna, M. (2009). Communitybased DOT-HAART Accompaniment in an Urban Resource-Poor Setting. AIDA Behavior, Apr 16. [Epub ahead of print]. Retrieved from the FIU PubMed Database.

Nachega, J., Leisegang, R., Bishai, D., Nguyen, H., Hislop, M., Cleary, S., et al. (2010). Association of antiretroviral therapy adherence and health care costs. Annals of Internal Medicine, 152(1), 18-25. Retrieved from the FIU CINAL Plus database.

Nakato, H., Vivancos, R. \& Hunter, P. R. (2007). A Systematic Review and Meta- analysis of the Effectiveness and Safety of Atovaquone / Proquanil (Malarone) for Chemoprophylaxis Against Malaria. Antimicrobial Chemotherapy, 60(5), 929-36. Retrieved May 28, 2009, from the FIU PubMed Database.

National Council on Patient Information and Education (NCPIE). (2007). Enhancing prescription medicine adherence: A national action plan. Retrieved from http://www.talkaboutrx.org/documents/enhancing_prescription_medicine_adherence.pdf

Nunnaly, J., \& Bernstein, I. (1994). Psychometric theory. Sydney: McGraw-Hill.

Ohrt, C., Richie, T. L., Widjaja, H., Shanks, G.D., Fitriadi, J., Fryauff, D. J., et al. (1997). Mefloquine Compared with Doxycycline for the Prophylaxis of Malaria in Indonesian Soldiers. A Randomized, Double-blind, Placebo-controlled trial. Annals of Internal Medicine, 126(12), 963-972. Retrieved May 28, 2009, from the FIU PubMed Database.

Okeck, B., Mwobobia, I., Muiruri, S., Mutiso, N., Myambura, J., \& Mwatele, C. (2008). Use of Integrated Malaria Management Reduces Malaria in Kenya. PLoS ONE, 3(12), e4050. Retrieved from the FIU PubMed Database.

Ollivier, L., Michel, R., Carlotti, M.P., Mahe, P., Romand, O., Todesco, A.,...Boutin, J.P. (2008). Chemoprophylaxis compliance in a French battalion after returning from a malaria endemic area. Journal of Travel Medicine, 15(5), 355-7. Retrieved from the FIU PubMed Database.

Olejnik, S., Li, J., Supattathum, S., \& Huberty, C.J. (1997). Multiple testing and statistical power with modified Bonferroni procedures. Journal of educational and behavioral statistics, 22, 389-406. Retrieved from the FIU CINAL Plus database.

Osterberg, L., \& Blaschke, T. (2005). Adherence to medication. The New England Journal of Medicine, 353(5), 487-97. Retrieved the New England Journal of Medicine website at http://content.nejm.org/cgi/reprint/353/5/487.pdf

Pagel, M. (2010). Key Principles for Creating Survey Questionnaire. Michael J. Pagel. As partial credit for coursework produced during PHC 7982, Pre-Dissertation Research, Fall 2010 at the Florida International University. Copy available upon request from cmbtdvr@hotmail.com.

Panagakou, S., Theodoridou, M., Papaevangelou, V., Papastergiou, P., Syrogiannopoulos, G., \& Goutziana, G. (2009). Development and assessment of a questionnaire for a descriptive cross - sectional study concerning parents' knowledge, attitudes and 
practices in antibiotic use in Greece. Bio Med Central Infectious Diseases, 9(52), 1-13. doi: 10.1186/1471-2334-9-52

Patel, A.K., Patel, K., \& Pate, J. Lactic Acidosis in HIV-1 infected patients receiving antiretroviral therapy. (2004). Journal of the Association of Physicians of India, 52, 666-69. Retrieved from http://www.japi.org/august2004/CR-666.pdf

Paulander, J., Olsson, H., Lemma, H., Getachew, A., \& San Sebastian, M. (2009). Knowledge, attitudes and practice about malaria in rural Tigray, Ethiopia. Global Health Action 2009. doi: 10.3402/gha.v2i0.1839

Payne, S. (1951). The Art of Asking Questions. Princeton, NJ: Princeton University Press.

Protopopescu, C., Raffi, F., Roux, P., Reynes, J., Dellamonica, P., Spire, B.,...Carrieri, M.P. (2009). Factors associated with non-adherence to long-term highly active antiretroviral therapy: a 10 year follow-up analysis with correction for the bias induced by missing data. Journal of Antimicrobial Chemotherapy, 64(3), 599-606. Retrieved from the Journal of Antimicrobial Chemotherapy website at http://jac.oxfordjournals.org/content/64/3/599.full.pdf+html

RAND Health. (2009). A review of barriers to medication adherence: A framework for driving policy options. Retrieved from http://www.rand.org/pubs/technical_reports/2009/RAND_TR765.pdf

Reif, S., Whetten, K., Ostermann, J., \& Raper, J.L. (2006). Characteristics of HIV-infected adults in the Deep South and their utilization of mental health services: A rural versus urban comparison. AIDS Care, 18(Suppl. 10-17). Abstract retrieved from the FIU PubMed database.

Remy, M., Ollivier, L., Meynard, J.P., Guette, C., Migliani, R., \& Boutin J.P. (2007). Outbreak of malaria among policemen in French Guiana. Military Medicine, 172:977-81 Retrieved from the FIU PubMed Database.

Resseguier, N., Machault, V., Ollivier, L., Orlandi-Pradines, E., Texier, G., Pradines, B.,...Rogier, C. (2010). Determinants of compliance with malaria chemoprophylaxis among French soldiers during missions in inter-tropical Africa. Malaria Journal, 9(41), 111. Retrieved from http://www.malariajournal.com/content/9/1/41

Rizzo, J., Abbott, T., \& Pashko, S. (1996). Labour productivity effects of prescribed medicines for chronically ill workers. Health Economics, 5, 249-65. Retrieved from Science Direct Journals database.

Robson, S. (2010, March 14). Troops with malaria could face punishment for disobeying orders. The Stars and Stripes on the web. Retrieved April 9, 2010 fromhttp://www.stripes.com/article.asp?section=104\&article $=68663$

Rowe, A., Claessens, A., Corrigan, R.A., \& Arman, M. (2009). Adhesion of Plasmodium falciparum-infected erythrocytes to human cells: molecular mechanisms and therapeutic 
implications. Expert Reviews in Molecular Medicine, 1(e16), doi:10.1017/S1462399409001082, 1-29

Sanofi-aventis U.S. LLC. (2008). Aralen - chloroquine phosphate FDA Official Human Prescription Drug Label. Retrieved from

http://dailymed.nlm.nih.gov/dailymed/druglnfo.cfm?id=8803

Sanofi-Synthelabo Inc. (2007). Primaquine Phosphate tablet FDA Official Human Prescription Drug Label. Retrieved from http://dailymed.nlm.nih.gov/dailymed/druglnfo.cfm?id=8315\&CFID=47855487\&CFTOKE $\mathrm{N}=$ ab5cf93654e9b0ce-0695DF3B-E9DA-290FD0E3A2698ECE875D\&jsessionid=ca30f2f23de253524d33

Sarbib, J., Nankani, G., \& Patel, P. (2006). The Booster Program for Malaria Control: putting knowledge and money to work. The Lancet, 268, 253-57. Retrieved from the FIU CINAL Plus database.

Sarna, A., Luchter, S., Geibel, S., Chersich, M.F., Munyao, P., Kaai, S.,...Rutenberg, N. (2008). Short- and long-term efficacy of modified directly observed antiretroviral treatment in Mombasa, Kenya: A randomized trial. Journal of Acquired Immune Deficiency Syndrome, 48(5), 611-9. Retrieved from the FIU PubMed database.

Shadish, W., Cook, T., Campbell, D. (2002). Experimental and Quasi-Experimental Designs for Generalized Causal Inference. New York: Houghton Mifflin Company.

Shanks, G. D., Edstein, M. D. (2005). Modern Malaria Chemoprophylaxis. Drugs, 65(15), 20912110. Retrieved May 28, 2009, from the FIU PubMed Database.

Sharma, S., Durand, R., \& Gur-Arie, O. (1981). Identification and Analysis of Moderator Variables. Journal of Marketing Research, 18(3), 291-300. Retrieved the JSTOR database at http://www.jstor.org/stable/3150970

Sherbourne, C., Hays, R., Ordway, L., DiMatteo, M., \& Kravitz, R. (1992). Antecedents of adherence to medical recommendations: Results from the Medical Outcomes Study. Journal of Behavioral Medicine, 15, 447-468. Abstract retrieved from the FIU PubMed database.

Six steps to compliance monitoring success. (2010, April). IRB Advisor. Retrieved from the FIU CINAL Plus database.

STAT RX USA LLC. (2010). Doxycycline (doxycycline hyclate) capsule FDA Official Human Prescription Drug Label. Retrieved from http://dailymed.nlm.nih.gov/dailymed/druglnfo.cfm?id=15291

Strickland, G. (2000). Hunter's Tropical Medicine and Emerging Infectious Diseases, 8th Edition. Philadelphia: W.B. Saunders Company. Retrieved from the FIU MD Consult database.

Sudman, S. \& Bradburn, N.M. (1983). Asking Questions: A Practical Guide to Questionnaire Design. San Francisco: Jossey-Bass Publishers. 
Taoufiq, Z., Gay, F., Balvanyos, J., Ciceron, L., Tefit, M., Lechat, P., \& Mazier, D. (2008). Rho kinase inhibition in severe malaria: Thwarting parasite-induced collateral damage to endothelia. Journal of Infectious Disease, 197, 1062-73. Retrieved March 11, 2010 from the Chicago Journals database at http://www.journals.uchicago.edu/doi/abs/10.1086/528988

Todd, G., Hpperus Buma, A., Green, M., Jaspers, C., 7 Lobel, T. (1997). Comparison of whole blood and serum levels of mefloquine and its carboxylic acid metabolite. American Journal of Tropical Medicine and Hygiene, 57(4), 399-402. Retrieved from the FIU Pub Med database.

United States Army, Headquarters (USA HQ). (2007). Army Regulation 40-501, Standards of Medical Fitness. Retrieved from http://www.apd.army.mil/pdffiles/r40_501.pdf

United States Army, Headquarters (USA HQ). (2009). Soldier's Manual of Common Tasks, Warrior Skills Level 1. Retrieved from http://www.25idl.army.mil/commontasks.pdf

United States Army, Headquarters (USA HQ). (2010). Army Training Circular, 3-22.20, Army Physical Readiness Training. Retrieved from https://armypubs.us.army.mil/doctrine/DR_pubs/dr_aa/pdf/tc3_22x20.pdf

United States Army Combat Readiness/Safety Center (US Army CRSC). (2010). FY 2005-2010, a query of the Army safety management information system (ASMIS), conducted on August 25, 2010 for Army class A-C negligent discharge accidents, range/target firing with unintended impact of round accidents, and other firing weapons accidents occurring from 1 October 2004, through 24 Aug 2010. Data retrieved August 30, 2010 via email correspondence with Mr. Alan Longshore, Chief of Operations.

United States Army Forces Central Command (USARCENT). (2006). USARCENT Policy Memorandum for Malaria Chemoprophylaxis. Fort McPherson, GA: Author. Retrieved June 03, 2010 from http://www.pdhealth.mil/downloads/ ThirdUSArmyUSARCENTCFLCCPolicyMemorandumSUR-01.pdf

United States Army Public Health Command (USAPHC). (2010). Daily Reportable Medical Events Summary for Wednesday-Sunday, November 24-28, 2010. PDF document retrieved via email correspondence with Major Scott Gilpatrick. Copy available upon request from cmbtdvr@hotmail.com.

United States Army Special Operations Command (USASOC), Information. (2011a). SOF Primer. Retrieved March 15, 2011 from http://www.soc.mil/USASOC\%20Headquarters/SOF\%20Primer.html

United States Army Special Operations Command (USASOC), Information. (2011b). Without equal: The story of Army Special Operations. Retrieved March 15, 2011 from http://www.soc.mil/USASOC\%20Headquarters/SOF\%20Story.html

United States Army Special Operations Command (USASOC), Information. (2012a). 75th Ranger Regiment Organizations. Retrieved April 24, 2012 from http://www.soc.mil/75th\%20Ranger\%20Regiment/Organization.html 
United States Army Special Operations Command (USASOC), Information. (2012b). SOF Story. Retrieved April 24, 2012 from

http://www.soc.mil/USASOC\%20Headquarters/SOF\%20Story.html

United States Department of Health and Human Services (DHHS). (2009). Guidelines for the Use of Antiretroviral Agents in HIV-1-Infected Adults and Adolescents. Retrieved July 12, 2010, from http://aidsinfo.nih.gov/contentfiles/AdultandAdolescentGL.pdf

United States Southern Command (USSOUTHCOM). (2004). Memorandum for Director, Joint Interagency Task Force-South, Information Paper. Miami, FL: Author. Retrieved June 03, 2010 from www.jiatfs.southcom.mil/...JIATF-

S\%20Medical\%20Readiness\%20Policy\%20Info\%20Paper.doc

West-ward Pharmaceutical Corp. (2010). Mefloquine Hydrochloride tablet FDA Official Human Prescription Drug Label. Retrieved from http://dailymed.nlm.nih.gov/dailymed/druglnfo.cfm?id=17794

Whitman, T., Coyne, P., Magill, A., Blazes, D., Green, M., Milhous, W,...Martin, G. (2010). An Outbreak of plasmodium falciparum malaria in U.S. Marines deployed to Liberia. The American Journal of Tropical Medicine and Hygiene, 83(2), 258-65. doi:10.4269/ajtmh.2010.09-0774

World Bank (WB). (2005). Framework for action, booster program for malaria control in Africa. Working Paper. Retrieved from http:// siteresources.worldbank.org/INTAFRBOOPRO/Resources/framework_for_action.pdf

World Health Organization (WHO). (2003). Adherence to long-term therapies: Evidence for action. Retrieved from http://www.who.int/chp/knowledge/publications/adherence_report/en/index.html

World Health Organization (WHO). (2004). Chronic HIV Care with ARV Therapy - Integrated management of adolescent and adult illness. Retrieved from http://www.who.int/3by5/publications/documents/en/IMAI_chronic.pdf

World Health Organization (WHO). (2008a). World Malaria Report 2008. Retrieved from http://www.who.int/malaria/publications/atoz/9789241563697/en/index.html

World Health Organization (WHO). (2008b). Advocacy, communication and social mobilization for TB control: a guide to developing knowledge, attitude and practice surveys. Retrieved from http://whqlibdoc.who.int/publications/2008/9789241596176_eng.pdf

World Health Organization (WHO). (2009). World Malaria Report 2009. Retrieved January 29, 2010, from http://www.who.int/malaria/world_malaria_report_2009/en/index.html

World Health Organization (WHO). (2010). World Malaria Report 2010. Retrieved February 21, 2011, from http://www.who.int/malaria/world_malaria_report_2010/en/index.html

Zelterman, D. (2006). Models for Discrete Data. New York: Oxford University Press 
APPENDICES 
Appendix 1.1 - Ethical Justification with Study Cost Illustration 


\section{Ethical Justification with Study Cost Illustration}

In light of the current budgetary crisis, structural and organizational realignments, as well as the many other cost cutting changes currently ongoing within this nation's military; an ethical justification for this study was deemed appropriate to help justify any oversight committee evaluating the use of government funds or the potential burdens that might be placed on highly trained and active group of U.S. Army special operations members. The justification employed Gostin's (2003) five-step process of validation for this justification, which was systematically applied to the study.

This justification was prepared well in advance of any approval process obtained for this study and presented to U.S. Army leadership in a very condensed form; which was rather persuasive. I believe this justification is an excellent testament to how public health research can be effectively and efficiently utilized with government assets and funds.

\section{Gostin's 5-step process}

Determining when it may be appropriate to intervene in a population is not always perfectly clear, and the means of determining this is not an absolute science. This is often the case with ethical issues. Thanks to Gostin, a concise and rather simple 5-step tool is provided to help ethically justify public health interventions. This method focuses on insuring the intervention does not compromise any personal or economic interests in the pursuit of improving public health (Gostin, 2003). If each of these five steps are considered satisfied by the investigator or approving authority, an endeavor may be considered ethically justified. Gostin acknowledges that these are only one set of factors to consider. There may be others that are equally important to consider and none should be considered absolute to an ethical justification.

The steps in Gostin's process include:

1. Demonstrate there is a true risk.

2. Demonstrate the intervention is effective. 
3. Assess the economic cost

4. Assess the burdens on human rights.

5. Assess the fairness of the intervention.

Each of the five steps above is has been thoroughly covered in detail within the complete ethical justification, which will be made available upon request sent to cmbtdvr@hotmail.com. Nevertheless, it is the opinion of the primary investigator, as well as the military approving authorities who have also reviewed this justification, that this study is satisfactorily justified.

Two important issues concerning the economic costs associated with this justification have been specifically maintained here; the remaining details can be requested if necessary.

First, the initial cost for this research was estimated at nearly $\$ 22,000$, while the actual total cost was only $\$ 14,230.79$. This was over $\$ 7,000$ less than the initial estimate.

Secondly, the minimum estimated costs of transporting and hospitalizing a patient infected with malaria were much greater than any estimation of costs associated with conducting this research. For example, The cost of a U.S. government medical evacuation aircraft can range anywhere from $\$ 2,000$ to $\$ 23,000$ per hour (Congressional Research Service, 2007; Department of State, 2005; General Accounting Office 2000); therefore, estimating at a very modest 4-hour one-way flight time, the travel costs alone of only two medical evacuations out of Afghanistan for treatment of malaria in a European hospital would cost a minimum of $\$ 32,000$. This calculation is based on 16 -hours of round trip flight time (8-hours minimum flight time to and from Afghanistan, multiplied times two). It must also be noted that this is an extreme minimum estimation, most flight routes are considerably longer and the aircraft that are most often used much more costly than the minimum cost used in this estimation.

In summation, the cost of one evacuation could easily be at or greater than $\$ 32,000$. The conduct of this research cost only $\$ 14,230$. In other words, including the cost of this research, if 
this research prevented only one case of malaria it could save nearly $\$ 18,000$ in the evacuation costs of that one case of malaria alone. This most certainly does not include any future medical costs, or the pain and future suffering that cannot be measured in exact costs. One final note: If only one evacuation for treatment of malaria was thwarted by this research, it would pay for itself two times over. 
Appendix 1.2 - Theoretical Models and Frameworks Identified in the Literature 
Theoretical Models and Frameworks Identified in the Literature

\begin{tabular}{|c|c|c|}
\hline Model & Impact/Relevance & $\underline{\text { Author }}$ \\
\hline Psychological Defense Model & Illness Recognition Failure & Forbes \\
\hline Cognitive Deficit Model & Decreased exec. Function & Forbes \\
\hline Theory of Chem. Dysregulation & Chemical Dysregulation & Forbes \\
\hline Neuropsychological Deficit Model & Damage to Brain Tissue & Forbes \\
\hline Awareness-to-adherence Model & Clinician Awareness to Adherence & Bosworth \\
\hline Health Decision Model & Identification of Patient Characteristics & Bosworth \\
\hline IMB Model & Improve Info./Motivation to Affect Behavior & WHO, 2003 \\
\hline Conservation of Resources Model & Improve Coping Capacity & DiClemente \\
\hline Self-Efficacy Theory & Cognitive Behavior/Reinforcement & Osterberg \\
\hline Adherence Loop Model & Role of Beliefs, Knowledge, and Action & Klein \\
\hline Theory of Reasoned Action & Improve Patient's Intention to Adhere & Martin \\
\hline Theory of Planned Behavior & Improve Patient's Intention to Adhere & Martin \\
\hline Transtheoretical/SOC Model & Patient's Intention to Adhere/Stages & Martin \\
\hline Social Cognitive Theory & Promote Behavior Capability, Benefits & Friedman \\
\hline Biomedical Perspective Model & Patient is Passive Follower of Dr's Orders & WHO, 2003 \\
\hline Behavioral/ Learning Theory & Importance of Pos./Neg. Reinforcement & WHO, 2003 \\
\hline Communication Perspective & Improve Provider Communication Skills & WHO, 2003 \\
\hline Protection-Motivation Theory & Patient concept of health threats/appraisal & WHO, 2003 \\
\hline Self Regulatory Model & Threat Conceptualization, Ideas, Coping & WHO, 2003 \\
\hline Multiplicative Model & Psychosocial Factor Relationship & Cleemp \\
\hline
\end{tabular}


Appendix 3.1 - USASOC Health Behavior KAP Questionnaire 


\section{RANGER \\ 3 in \\ RANGER \\ 75. \\ USASOC \\ Health Behavior \\ KAP Questionnaire}




\title{
USASOC Health Behavior KAP Questionnaire
}

\author{
Primary Investigator: Major Michael J Pagel \\ Military Affiliation: The Walter Reed Army Institute of Research, \\ US Army Public Health Command
}

Academic Affiliation: PhD Student, Health Promotion and Disease Prevention

Florida International University; Miami, Florida

Contact Info: Phone: 786-255-3132, Email: michael.pagel1@us.army.mil

Study Period: July 01, 2011 thru August 30, 2012

Approximate Questionnaire Completion Time: 90 minutes

\author{
Demographic and Malaria Chemoprophylaxis questions \\ Total Questions: 133 Questions \\ Approximate time to complete this section during reviews: $\underline{30 \mathrm{~min}}$ \\ Condom Use questions \\ Total Questions: 109 Questions \\ Approximate time to complete this section: $\underline{30 \mathrm{~min}}$ \\ Weapon Safety questions \\ Total Questions: 104 Questions \\ Approximate time to complete this section: $\underline{30 \mathrm{~min}}$ \\ Administrative questions \\ Total Questions: $\underline{3}$ Questions
}

Thank you for participating in this questionnaire survey. The information you give in this survey will help fill the gap of knowledge the US Army has on health behaviors within the USASOC populations, specifically the Ranger units. The public health community appreciates your support and wants to

ensure you that you are contributing to improving the health of all Ranger and other Special Operations members.

Your participation in this survey is completely voluntary.

Your answers will be kept confidential.

You will remain anonvmous and there will be no means of identifying or matching you to any of the answers

you may provide.

You must be 18 vears or older to proceed.

The following pages contain questions and statements dealing with the knowledge, attitudes, and practices concerning three health behaviors. These three behaviors are (1) taking malaria chemoprophylaxis medications, (2) using condoms, (3) maintaining weapon safety. Knowledge is simply what you know about the behavior. Attitudes are simply your ideas, thoughts, or feelings about the behavior. Practices are simply what you, yourself do in relation to what you know and how you feel about any of the three behaviors. There are also questions about communication, unit level education, unit level training, mass media, and command support.

Please feel free to ask for assistance from a research team member if you have any questions, or need any assistance.

$\begin{array}{lll}\text { RV340 } & 2 & \text { Version } 1.2 \\ \text { Questionnaire Cover Sheets } & 2 & 12 \text { Aug2011 }\end{array}$




\section{Questionnaire Completion Instructions}

\section{Entering responses:}

-Please answer"each" question.

-All entries must be made with black or blue ballpoint pen (do not use pencil).

-All entries must be marked with an "X" in applicable circle fields. Example:囚

\section{Making corrections:}

-Do not erase, do not use correction fluid.

-Draw a single line through the incorrect information. Example:

\section{Please Note:}

-Each question must have a response. "Refuse to answer", "Don't Know", and

"Not Applicable" fields are offered in case you cannot or will not respond.

-If you are confused by a question, or need assistance in any way,

please inform the study personnel administering the questionnaire. 


\section{Demographics}

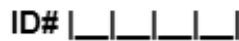

First, we would like to ask some general information that is necessary to properly analyze what we obtain and make it meaningful to military populations.

D1. Could you please let us know the Ranger unit you are currently assigned to?

Remember, this information will not be traceable back you, or linked to you as an individual in any way. This information will only let us know if there are any specific health related issues associated with the unit of assignment.
O 1. Ranger Regimental Headquarters
O 4. $2^{\text {nd }}$ Ranger Battalion
O 2. Ranger Special Troops Battalion
Q 5. $3^{\text {rd }}$ Ranger Battalion
O 3. $1^{\text {st }}$ Ranger Battalion
Refuse to Answer

D2. What is your gender?
O 1. Male
O 2. Female
O Refuse to Answer

D3. What is your current age?
O $1.18-21$
2. $22-25$
O 3. $26-30$
O $4.31-35$
O $5.36-40$
O 6. Over 40 years old
O Refuse to Answer

D4. What is the highest level of education you yourself have completed?
O 1. Less than high school, no equivalency exam
O 2. High school equivalency exam
O 6. Bachelor degree or higher
3. High school graduate
O 4. Some college
Don't Know
O 5. Associates degree

D5. What is your current marital status? (Please mark only one "best" answer).

O 1. Currently Married, monogamous relationship

O 2. Currently Married, dating others

O 3. Single (Never Married), monogamous relationship

Q 4. Single (Never Married) dating others

5. Currently Divorced, monogamous relationship

O 6. Currently Divorced, dating others

O 7. Widowed or Widower, monogamous relationship
8 8. Widowed or Widower, dating others

8 9. Single, Divorced, or Widowed/Widower; not dating others

O 10. Legally Separated from spouse, not dating

O 11. Legally Separated from spouse, dating others

O Refuse to Answer

Other 
D6. What military rank do you currently hold?

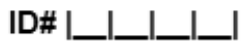
O 1. E1 thru E4 (not including Corporal)
O 5. $01-03$
2. E4 Corporal thru E6
O 6. 04-010
Oे 3. E7-E9
O Refuse to Answer
4. W1-W5

D7. Are you currently in a leadership position?
O 0 . No
O Refuse to Answer
1. Yes

D8. Which category "best" fits your military occupational specialty? (Mark only one.)
1. Infantry
2. Artillery
3. Medical (including veterinary and dental)
O. Armor
5. Logistics
6. Intelligence

О $\begin{aligned} & \text { 7. Weapons } \\ & \text { 8. Personnel } \\ & \text { ○. Engineer } \\ & \text { O̊. Communications } \\ & \text { 10. Cefuse to Answer } \\ & \text { Other }\end{aligned}$

D9. What year did you first enter the military?
(1. Prior to 1991
4. 2001 to 2005
2. 1991 to 1995
5. 2006 to Present
Ǒ 3. 1996 to 2000
Refuse to Answer

D10. Not counting breaks in service: How many years of active service do you yourself have?
1. Less than 1 year
2. 1 to 2 years
6. 11 to 15 years
3. 3 to 4 years
7. 16 to 20 years
4. 5 to 6 years
8. Over 20 years
O. 7 to 10 years
Refuse to Answer

D11. Have you served in any other branch of the military, besides the US Army?
0 . No
O Refuse to Answer
O 1. Yes

D12. How long have you been assigned to your current Ranger unit?

\begin{tabular}{|c|c|c|c|}
\hline & $\left\{\begin{array}{l}2.1 \text { to } 2 \text { years } \\
\text { 3. } 3 \text { to } 4 \text { years } \\
\text { 4. } 5 \text { to } 6 \text { years } \\
\text { 5. } 7 \text { to } 10 \text { years }\end{array}\right.$ & $\begin{array}{l}\text { 6. } 11 \text { to } 15 \text { years } \\
\text { 7. } 16 \text { to } 20 \text { years } \\
\text { 8. Over } 20 \text { years } \\
\text { Refuse to Answer }\end{array}$ & \\
\hline $\begin{array}{l}\text { RV340 } \\
\text { Questionnaire }\end{array}$ & & 5 & $\begin{array}{l}\text { Version } 1.2 \\
\text { 12Aug2011 }\end{array}$ \\
\hline
\end{tabular}




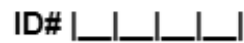

D13. Can you please give an "estimate" of your annual household income? This would include your US Army salary, wages and earnings of your significant other, and any other income that contributes to support your household. (In US Dollars please.)
O 1. $0-10,000$ range
O 8. $95,000-110,000$ range
2. $10,000-20,000$ range
9. $110,000-125,000$ range
O. 3. $20,000-35,000$ range
O 10. $125,000-150,000$ range
O. $35,000-50,000$ range
11. $150,000-200,000$ range
5. 50,000-65,000 range
O 12. $200,000-250,000$ range
8. 6. $65,000-80,000$ range
8 13. Over 250,000
7. $80,000-95,000$ range
Refuse to answer

D14. Do you have any biological or non-biological children you care for or support?
O 0 . No
O Refuse to Answer
O 1. Yes

D15. Which religion do you best identify with?

O 1. Roman Catholic

O 4. Any other Religion

O 2. Jewish

3. Christian (other than Catholicism)

O 5. Agnostic, Atheist, or Non-Religious

O Refuse to Answer

D16. What race or ethnicity do you most closely identify yourself with? (Choose only one answer. If not specifically listed, you may choose the Other Races or Ethnicities category).
O 1. Black or African American
O 4. Other Races or Ethnicities
Q 2. Hispanic or Latino
O Refuse to Answer
3. White

D17. Have you yourself ever deployed to a foreign country?
O 0 . No
O Don't Know
O 1. Yes
O Refuse to Answer

\begin{tabular}{lll}
\hline RV340 & 6 & Version 1.2 \\
Questionnaire & 6 & 12 Aug2011
\end{tabular}


ID\#|_ا_ا_ا_|

\section{General Knowledge of Malaria and Chemoprophylaxis Medications}

M18. The next set of questions considers your knowledge regarding malaria and malaria chemoprophylaxis medications.

For each question, please provide the "single" best answer. If you don't know the answer to the question, it is alright to say that you don't know. You may also refuse to answer the question. Do not hesitate to ask for help if you do not understand.

M18A. Have you ever heard of malaria?
O. No
Don't Know
1. Yes
Refuse to Answer

M18B. Can a person get malaria from a mosquito bite?
0. No
Don't Know
○ 1. Yes
O Refuse to Answer

M18C. Can a person get malaria from blood transfusions?
O. No
Don't Know
○ 1. Yes
Refuse to Answer

M18D. Can malaria be transmitted from person to person by close contact or cough?
O. No
Don't Know
O 1. Yes
O Refuse to Answer

M18E. Can malaria cause death?
O. No
Don't Know
1. Yes
O Refuse to Answer

M18F. Have you ever been prescribed malaria chemoprophylaxis medications?
O. No
Don't Know
1. Yes
Refuse to Answer

\begin{tabular}{lll}
\hline RV340 & 7 & Version 1.2 \\
Questionnaire & & 12 Aug2011
\end{tabular}


M18G. What do malaria chemoprophylaxis medications do? (Please choose only one best answer.)
O 1. Prevent mosquito bites
O Don't Know
O 2. Prevent malaria from developing in your body
O Refuse to Answer
O 3. Prevent both HIV and Malaria
O 4. Make you permanently immune to Malaria

$\mathrm{M} 18 \mathrm{H}$. Which medication is "not" a malaria chemoprophylaxis medication?

O 1. Mefloquin

O 2. Doxycycline

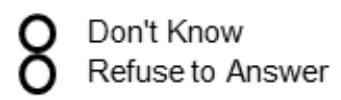

O 3. Penicillin

O 4. Chloroquine

M18I. Can malaria chemoprophylaxis medications be stopped immediately after leaving the area where malaria is present?
O 0 . No
O Don't Know

M18J. Can missing a single dose of malaria chemoprophylaxis medications put you at greater risk for contracting malaria?
O 0. No
O Don't Know
O 1. Yes
O Refuse to Answer

M18K. Which form of malaria prevention is the most effective?
O 1. There is no effective form of Malaria prevention
O $\begin{aligned} & \text { Don't Know } \\ & \text { Refuse to Answer }\end{aligned}$
O 2. Taking Malaria Chemoprophylaxis Medications
O 3. Avoidance with repellents, nets, and protective clothing
O 4. Combination of Medications and Avoidance

M18L. Can the military punish you for not taking your malaria chemoprophylaxis medications as recommended?
O 0 . No
O Don't Know
O 1. Yes
O Refuse to Answer

\begin{tabular}{lrr}
\hline RV340 & 8 & Version 1.2 \\
Questionnaire & & 12 Aug 2011
\end{tabular}


M18L2. If there are any punishments or repercussions: Can you please choose which of the following punishments or repercussions you think would be the most likely to occur? Please choose only one.

O 1. Article 15 , with reduction in rank

2. Article 15, with loss of some pay

O. 3. Court martial proceedings

O Don't Know

O 4. Some type of limited punishment, such as extra duty

Not Applicable, I don't think there are any punishments

O Refuse to Answer

M18M. In the past 36 months: Have you yourself been deployed to an area where malaria was present?

Oे 0 . No

O Don't Know

O 1. Yes

O Refuse to Answer

M18M1. In the past 36 months: Have you yourself been deployed to Afghanistan, Pakistan, India, any country in South or Central America, Haiti or the Dominican Republic, any country in Africa, or any country in Southeast Asia?
O 0. No
O Don't Know
O 1. Yes
O Refuse to Answer

M18N. How long were you actually in the area where there was a risk of contracting malaria?
O 1. 1-7 days
O 6. Between 1 to 2 years
O 2. 8-30 days
O 3. $31-90$ days
O. 4. $91-180$ days
O 5.181 to 365 days
O 7. More than 2 years
O Don't Know
Not Applicable, I was never deployed to an area with Malaria
O Refuse to Answer

M180. Have you yourself known of any people, including civilians, being infected with malaria in places where you have been deployed?
O 0 . No
O Don't Know
O 1. Yes
Refuse to Answer

M18P. Can you get sick from malaria after you have returned to your home unit back in the US?
O 0 . No
O $\begin{array}{ll}\text { Don't Know } \\ \text { Refuse to Answer }\end{array}$

$\begin{array}{lll}\text { RV340 } & 9 & \text { Version } 1.2 \\ \text { Questionnaire } & & 12 \text { Aug2011 }\end{array}$




\section{ID\# |_ا_ا_ا_|}

\section{General Attitudes towards Malaria Chemoprophylaxis Medications}

M19. The next set of questions consider your general attitudes, opinions, and ideas about malaria chemoprophylaxis medications.

Please answer each question to the best of your ability with the "single" best answer. Do not hesitate to ask for help if you do not understand.

M19A. In your opinion: When you were prescribed malaria chemoprophylaxis medications, were you well informed on "how" to take them?
O No
Don't Know
○ 1. Yes
Refuse to Answer
Not Applicable, I was never prescribed this medication

M19B. In your opinion: Were you well informed on "why" it was important to take your malaria chemoprophylaxis medications regularly?
O. No
Don't Know
1. Yes
Refuse to Answer

M19C. Does it bother you to have to take a medication when you are "not" sick?
O 0 . No
Don't Know
1. Yes
Refuse to Answer

M19C1. If yes: How much does it bother you? (Answer "Not Applicable" if it does not apply to you)
1. Not much
2. Moderately
Not Applicable, it does not bother me
Ô. Very much
Refuse to Answer

M19D. Does it bother you to have to take pills?
0. No
Don't Know
1. Yes
Refuse to Answer

M19D1. If yes: How much does it bother you? (Answer "Not Applicable" if it does not apply to you)
1. Not much
2. Moderately
Not Applicable, it does not bother me
3. Very much
Refuse to Answer

M19E. Do you yourself think there are "unpleasant" side effects, or other issues with malaria chemoprophylaxis medications that may cause you "not" to take them as directed?

\begin{tabular}{llll} 
O & $\begin{array}{l}\text { 0. No } \\
\text { 1. Yes }\end{array}$ & O & $\begin{array}{l}\text { Don't Know } \\
\text { Refuse to Answer }\end{array}$ \\
\hline $\begin{array}{l}\text { RV340 } \\
\text { Questionnaire }\end{array}$ & & 10 & Version 1.2 \\
12 Aug2011
\end{tabular}


ID\# |_ا_ا_ا_|

M19E1. If yes: How serious are they? (Answer "Not Applicable" if it does not apply to you)
O 0. Not serious
O Not Applicable
1. Moderately serious
O Refuse to Answer
O 2. Very serious

M19E2. If you think there are "unpleasant" side effects, or other issues with malaria chemoprophylaxis: Can you name those side effects or issues? (Answer "Not Applicable" if it does not apply to you)

O 0. No, I cannot define them

O 1. Yes- Please specify:
O Don't Know

O Not Applicable, I have no side effects or issues

O Refuse to Answer

M19F. Do you think taking malaria chemoprophylaxis medications on a daily basis is a hassle?
O 0 . No
O 1. Yes
O Don't Know
Oefuse to Answer

M19F1. If yes: How much of a hassle is it to take daily? (Answer "Not Applicable" if it does not apply to you)
O 1. Not much
O Not Applicable, it does not bother me
O. 2. Moderately
O Refuse to Answer
O 3. Very much

M19G. Do you think taking malaria chemoprophylaxis medications on a weekly basis is a hassle?
O 0 . No
O Don't Know
O 1. Yes
O Refuse to Answer

M19G1. If yes: How much of a hassle is it to take weekly? (Answer "Not Applicable" if it does not apply)

$\begin{array}{ll}\text { O } & \text { 1. Not much } \\ \text { 2. Moderately } & \text { 3. Very much }\end{array}$

O Not Applicable, it does not bother me.

Refuse to Answer

\begin{tabular}{lll}
\hline RV340 & 11 & Version 1.2 \\
Questionnaire & 12 Aug2011
\end{tabular}


ID\# |_ا_L_ا_L

M19H. Do you think about any punishments or other repercussions that may occur through the military if you do "not" take malaria chemoprophylaxis medications as directed?
O 0 . No
O Don't Know
O 1. Yes
O Refuse to Answer

M19H1. If yes: How bad do you think those punishments or repercussions will be? (Answer "Not Applicable" if it does not apply to you)
O 1. Mild
O Not Applicable, I don't think of any repercussions
Q 2. Moderate
O Refuse to Answer

M19I. Do those punishments or repercussions influence you to take your malaria chemoprophylaxis medications as directed?
O 0 . No
O Don't Know
O 1. Yes
Not Applicable, I don't think of any repercussions
Refuse to Answer

M1911. If yes: How much influence do they have? (Answer "Not Applicable" if it does not apply to you)
O 1. Not much
O Not Applicable, I don't think of any repercussions
O 2. Moderately
O Refuse to Answer

M19J. Do you feel that the advice given by medical personnel concerning the threat of malaria is "always" accurate?
O 0 . No
O Don't Know
O 1. Yes
O Refuse to Answer

M19K. When in a region thought to have malaria: Do you sometimes think you can determine the risk of malaria better than medical personnel, particularly when you do not find any cases of malaria, or see mosquitoes?
O 0 . No
O Don't Know
O 1. Yes
Refuse to Answer

M19L. When deployed to a combat area: Compared to other high priority tasks associated with combat, I "do not" believe the use of malaria chemoprophylaxis medications is important to protect me from malaria.
O 1. Strongly Disagree
O Not Applicable, I have not been to a combat area
O 2. Disagree
O Refuse to Answer
O 3. Agree
O 4. Strongly Agree

\begin{tabular}{lcc}
\hline RV340 & 12 & Version 1.2 \\
Questionnaire & & 12 Aug2011
\end{tabular}




\section{Descriptive Norms}

M20. The next set of questions consider "your" attitudes, opinions, and ideas about how "others" behave and think about malaria and malaria chemoprophylaxis medications.

Please answer each question to the best of your ability with the "single" best answer. Do not hesitate to ask for help if you do not understand.

M20A. When in a malaria region, or when planning to enter a region that is thought have malaria: How many of your friends or coworkers do you believe take their malaria chemoprophylaxis medications "exactly" as directed?
O 1. None
4. All
O 2. Some
Refuse to Answer
3. Most

M20B. How many of your friends or coworkers do you believe "do not" take their malaria chemoprophylaxis medications "exactly" as directed?
O 1. None
4. All
O 2. Some
Refuse to Answer
Oे. Most

M20C. When in a region, or when planning to enter a region that is thought have malaria: How many of your friends or coworkers do you believe feel they are actually at risk of catching malaria?
1. None
O 4. All
2. Some
Refuse to Answer
3. Most

M20D. How many of your friends or coworkers do you believe think that malaria is a disease that only "other" people will get, because they are too healthy to catch it?
1. None
4. All
2. Some
Refuse to Answer

M20E. How many of your friends or coworkers do you think feel that malaria is not a serious disease?
1. None
2. Some
O 3. Most

4. All

Refuse to Answer

\begin{tabular}{lcc}
\hline RV340 & 13 & Version 1.2 \\
Questionnaire & & 12 Aug2011
\end{tabular}


ID\# |_ا_ا_ا_ا_

M20F. When they are in a region thought to have malaria: How many of your friends or coworkers do you believe think about malaria on a "regular" (daily or weekly) basis?
O 1. None
O 4. All
Оิ 2. Some
O Refuse to Answer

M20G. When in a region, or when planning to enter a region with malaria: How many of your friends or coworkers do you believe add malaria, or malaria chemoprophylaxis medications into their regular mission planning process?
O 1. None
4. All
O 2. Some
O Refuse to Answer
O 3. Most

$\mathrm{M} 2 \mathrm{OH}$. In your opinion: How many of your friends or coworkers believe the information given by medical personnel concerning the threat of malaria is "always" accurate?
O 1. None
O 4. All
Oิ 2. Some
O Refuse to Answer

M20I. In your opinion: How many of your friends or coworkers believe the information given by medical personnel concerning the threat of malaria is "not" always accurate?
O 1. None
O 2. Some
O 4. All
O 3. Most
O Refuse to Answer

RV340 14

Version 1.2 


\section{Injunctive Norms}

M21. The next set of questions consider your attitudes, opinions, and ideas about how

"others" approve of what "you" do concerning malaria and malaria chemoprophylaxis medications.

Please answer each question to the best of your ability with the "single" best answer by indicating whether you strongly disagree, disagree, agree, or strongly agree with each statement or question. Do not hesitate to ask for help if you do not understand.

M21A. If I were in a place where malaria was a threat, my friends and coworkers would think that I "should" take my malaria chemoprophylaxis medications exactly as directed.
1. Strongly Disagree
Q 4. Strongly Agree
O 2. Disagree
Ǒ Refuse to Answer
Ǒ 3. Agree

M21B. My friends and coworkers think its "OK" for me to take my malaria chemoprophylaxis medications every so often, instead of exactly as directed.
1. Strongly Disagree
4. Strongly Agree
2. Disagree
Ǒ Refuse to Answer
O. Agree

M21C. My friends and coworkers would think it was "OK" if I went on a "multiple day" mission and "did not" take my malaria chemoprophylaxis medications with me.
1. Strongly Disagree
Oे 4. Strongly Agree
2. Disagree
Ǒ 3. Agree

M21D. My friends and coworkers think it's "OK" if I forget to take my malaria chemoprophylaxis medications every once in a while.
1. Strongly Disagree
O 4. Strongly Agree
Q 2. Disagree
Orefuse to Answer
Ǒ 3. Agree

M21E. My friends and coworkers would think it's "cool" if I decided not to take my malaria chemoprophylaxis medications.
1. Strongly Disagree
O 4. Strongly Agree
Q 2. Disagree

M21F. My friends and coworkers would think it was "OK" for me to suggest there was no threat of malaria because I did not see any evidence of it: despite what medical personnel were saying.

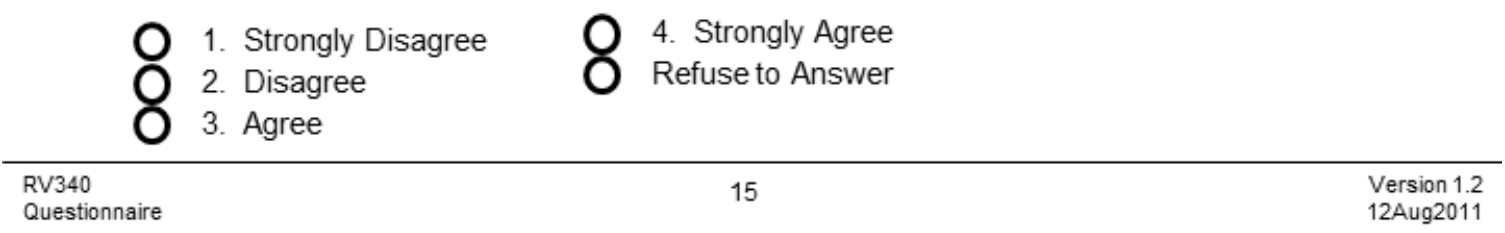


M21G. My friends and coworkers would think it's "OK" if I "lied" about taking my malaria chemoprophylaxis medications, even though I really did not take them.
O 1. Strongly Disagree
O 4. Strongly Agree
O 2. Disagree
Refuse to Answer
O 3. Agree

M21H. My friends and coworkers would think it's "OK" if I ordered someone to take their malaria chemoprophylaxis medications, even though that person "did not" want to take them.

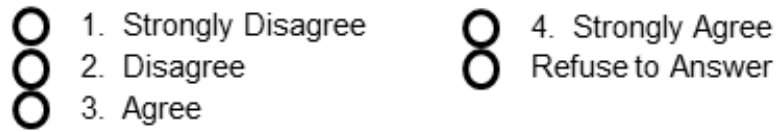

M211. My friends and coworkers would think it's "OK" for me to tell someone they did not have to take their malaria chemoprophylaxis medications if they "did not" want to do so, despite medical advice.
O 1. Strongly Disagree
O 4. Strongly Agree
O 2. Disagree
O Refuse to Answer
O 3. Agree

M21J. If I were a leader: My friends and coworkers would think I should set the example for others to follow, particularly when taking malaria chemoprophylaxis medications as directed
O 1. Strongly Disagree
O 4. Strongly Agree
Q 2. Disagree
O Refuse to Answer
O 3. Agree

M21K. If I were a leader: Others would think "negatively" about my character if they knew I was not taking my malaria chemoprophylaxis medications.
O 1. Strongly Disagree
O 2. Disagree
Q 4. Strongly Agree
O 3. Agree

M21L. If I were a leader: I would not want others to know that I was "not" taking my malaria chemoprophylaxis medications, because it might influence them "not" to take their medications.
O 1. Strongly Disagree
O 4. Strongly Agree
Q 2. Disagree
O Refuse to Answer
O 3. Agree

M21M. My friends and coworkers would "not" approve of my actions if I caught malaria because I did "not" take my malaria chemoprophylaxis medications.
O 1. Strongly Disagree
Q 4. Strongly Agree
O 2. Disagree
O Refuse to Answer
O 3. Agree

M21N. My friends and coworkers would "not" approve of my actions if I caught malaria and compromised a mission because I did "not" take my malaria chemoprophylaxis medications.

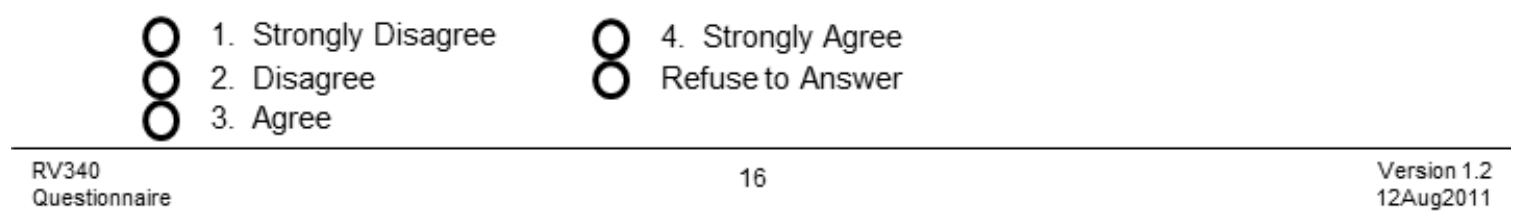




\section{Perceived Susceptibility and Risk}

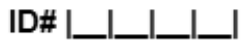

M22. You will now be asked to answer some questions about how you perceive the risk associated with malaria, and what you feel the likelihood is that you might get infected with malaria.

You may either be asked a question, or required to respond to a statement.

Please complete all to the best of your ability by choosing the "single" best answer. Do not hesitate to ask for help if you do not understand.

M22A. Do you believe malaria is a serious disease?
O 0 . No
O Don't Know
O 1. Yes
O Refuse to Answer

M22B. What is the likelihood that your friends and coworkers may ever contract malaria?

O 1. Not at all likely

O 2. Somewhat likely

O Not Applicable. My friends and coworkers

O็ 3. Very likely do not travel to areas with malaria

O Refuse to Answer

M22C. What is the likelihood that you will ever contract malaria?

O 1. Not at all likely

O 2. Somewhat likely

O Not Applicable, I have never traveled to areas with malaria

O 3 . Very likely

M22D. Do you feel that malaria is a disease that only others will contract?
O 0 . No
Don't Know
O 1. Yes
O Refuse to Answer

M22E. When you are in a region that has malaria: Do you think about malaria on a regular (daily or weekly) basis?
O 0 . No
O Don't Know
O 1. Yes
Not Applicable, I have never traveled to areas with malaria
O Refuse to Answer

M22F. If you were to see individuals around you who have contracted malaria: Do you think that would influence you to take your malaria chemoprophylaxis medications "exactly" as directed?
O 0 . No
O Don't Know
O 1. Yes
O Refuse to Answer

$\begin{array}{lll}\text { RV340 } & 17 & \text { Version } 1.2 \\ \text { Questionnaire } & & 12 \text { Aug2011 }\end{array}$




\section{Behavior Outcome Related Practices}

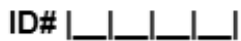

M23. This section of statements and questions consider the practices that may affect you taking or not taking malaria chemoprophylaxis medications.

You may either be asked a question, or required to respond to a statement. Please complete all to the best of your ability by choosing the "single" best answer. Do not hesitate to ask for help if you do not understand.

M23A. When you yourself were prescribed malaria chemoprophylaxis medications: Did you take them exactly as directed? (Please choose only answer one answer)
O 0 . No
O Don't Know
O 1. Yes
Not Applicable, I was never prescribed the medications
Refuse to Answer

M23B. How often did you take these medications? (Please answer one)

O. None of the time, Never

1. Some of the time, about $25 \%$ of the time

O 2. Most of the time, about $50 \%$ to $75 \%$ of the time

3. All the time, $100 \%$ of the time, Without exception
O Don't Know

O Not Applicable, I was never prescribed the medications

O Refuse to Answer

M23C. I only take my malaria chemoprophylaxis medications because I "fear the repercussions" that the military may bring upon me if I catch malaria while I am "not" taking them.

O. False (No)

O Don't Know

O 1. True (Yes)

Not Applicable, I was never prescribed the medications

Orefuse to Answer

M23D. I was not taking my medications as directed at first, but when I noticed individuals around me contracting malaria: I took my malaria chemoprophylaxis medications "exactly" as directed.

O. False (No)

O Don't Know

O 1. True (Yes)

Not Applicable, This never happened to me

Refuse to Answer

M23E. If you began taking your medications because you noticed individuals around you contracting malaria: Did you take the medicine even though you felt some side effects from the medicine? (Answer "Not Applicable" if it does not apply to you)
O 0 . No
Q Don't Know
O 1. Yes
Not Applicable, This never happened to me
Orefuse to Answer

$\begin{array}{lcc}\text { RV340 } & 18 & \text { Version } 1.2 \\ \text { Questionnaire } & & 12 \text { Aug2011 }\end{array}$


ID\# |_ا_ا_L

M23F. When I am reminded, I take my malaria medications. Otherwise, I often forget to take them.
O 0. False (No)
O 1. True (Yes)
O Don't Know
Not Applicable, I was never prescribed medications
O Refuse to Answer

M23G. When in a region thought to have malaria: I plan malaria chemoprophylaxis medications into my missions.

O. None of the time, Never

1. Some of the time, about $25 \%$ of the time

2. Most of the time, about $50 \%$ to $75 \%$ of the time

O 3. All the time, $100 \%$ of the time, Without exception
O Don't Know
Not Applicable, I was never prescribed the medications
Refuse to Answer

$\mathrm{M} 23 \mathrm{H}$. Do you plan to take your malaria chemoprophylaxis medications exactly as directed the next time they are prescribed to you?
0. No
1. Yes
Don't Know
Not Applicable
Refuse to Answer 
M24. This section considers communications you may have with others, that may affect you taking or not taking malaria chemoprophylaxis medications.

Please complete all to the best of your ability by choosing the "single" best answer. Do not hesitate to ask for help if you do not understand.

M24A. I discussed the need to take malaria chemoprophylaxis medic ations "as directed" with my friends and coworkers.
Oे $\begin{array}{ll}\text { 0. Never } \\ \text { 1. Once }\end{array}$
2. A few times
3. Many times
Orefuse to Answer

M24B. I discussed the decision "not" to take malaria chemoprophylaxis medications with my friends and coworkers.
O $\begin{array}{ll}\text { O. Never } \\ \text { 1. Once }\end{array}$
2. A few times
3. Many times
Orefuse to Answer

M24C. I advised my friends or coworkers "to take" their malaria chemoprophylaxis medications as directed.
○ $\begin{array}{ll}\text { O. Never } \\ \text { 1. Once }\end{array}$
2. A few times
3. Many times
Refuse to Answer

M24D. I advised my friends or coworkers "not" to take their malaria chemoprophylaxis medications.
O 0. Never
2. A few times
3. Many times
Orefuse to Answer

M24E. I discussed the threat of malaria with my friends and coworkers.
Oे $\begin{array}{ll}0 . & \text { Never } \\ \text { 1. Once }\end{array}$
2. A few times
3. Many times
Orefuse to Answer

M24F. I was influenced by my conversations with others "to take" my malaria chemoprophylaxis medications as directed.
O 0. Never
3. Many times

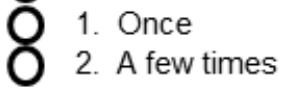
O̊ Not Applicable, I never had these conversations Refuse to Answer

M24G. I was influenced by my conversations with others "not" to take my malaria chemoprophylaxis medications.

\begin{tabular}{llll} 
& O $\begin{array}{l}\text { 0. Never } \\
\text { 1. Once } \\
\text { 2. A few times }\end{array}$ & O & $\begin{array}{l}\text { 3. Many times } \\
\text { Not Applicable, I never had these conversations } \\
\text { Refuse to Answer }\end{array}$ \\
\hline $\begin{array}{l}\text { RV340 } \\
\text { Questionnaire }\end{array}$ & & 20 & Version 1.2 \\
12 Aug2011
\end{tabular}


M25. This section considers education you may or may not have had concerning malaria and malaria chemoprophylaxis medications.

Please complete all to the best of your ability by choosing the "single" best answer. Do not hesitate to ask for help if you do not understand.

M25A. Have you "ever" had any education concerning the dangers of malaria?
O. Never
2. A few times
Ô 1. Once
3. Many times
Ohefuse to Answer

M25B. If you have had this education: Do you feel you are well informed because of it? (Answer "Not Applicable" if it does not apply to you)
0. No
1. Yes
Not Applicable, never offered to me
Oh Refuse to Answer

M25C. Have you "ever" had any education concerning the importance of taking malaria chemoprophylaxis medications as prescribed?
O $\begin{array}{ll}\text { 0. Never } \\ \text { 1. Once }\end{array}$
2. A few times
3. Many times
Refuse to Answer

M25D. If you have had this education: Do you feel you are well informed because of it? (Answer "Not Applicable" if it does not apply to you)
$\begin{array}{ll}\text { O } & \text { No } \\ \text { 1. Yes }\end{array}$
Not Applicable, never offered to me
Refuse to Answer

M25E. Is education on the dangers of malaria offered at your "current" unit?
O 0 . No
Don't Know
○ 1. Yes
Refuse to Answer

M25F. Do you feel this education informed you well?

(Answer "Not Applicable" if it does not apply to you)
О $\begin{array}{ll}\text { 0. No } \\ \text { 1. Yes }\end{array}$
O Not Applicable, never taken at my unit
Ǒ Refuse to Answer

$\begin{array}{lll}\text { RV340 } & 21 & \text { Version } 1.2 \\ \text { Questionnaire } & & 12 \text { Aug2011 }\end{array}$


M25G. Is education on the importance of taking malarial chemoprophylaxis medications as directed offered at your "current" unit?
0. No
1. Yes
Don't Know
Ǒ Refuse to Answer

M25H. Do you feel this education informed you well?

(Answer "Not Applicable" if it does not apply to you)
0. No
O Not Applicable, never taken at my unit
1. Yes
O Refuse to Answer

\section{Behavior Related Training}

M26. This section considers training you may or may not have had concerning malaria and malaria chemoprophylaxis medications.

Please complete all to the best of your ability by choosing the "single" best answer.

Do not hesitate to ask for help if you do not understand.

M26A. Have you "ever" had any training that demonstrated how to prevent malaria?
O. Never
2. A few times
3. Many times
Refuse to Answer

M26B. Do you feel this training prepared you well? (Answer "Not Applicable" if it does not apply to you)
O. No
1. Yes
Not Applicable, never offered to me
Refuse to Answer

M26C. Is training available at your "current" unit that demonstrates how to prevent malaria?
0. No
1. Yes
Don't Know
Refuse to Answer

M26D. Do you feel this training prepared you well? (Answer "Not Applicable" if it does not apply to you)
0. No
1. Yes
O Not Applicable, never taken at my unit
Ǒ Refuse to Answer

$\begin{array}{lll}\text { RV340 } & 22 & \text { Version } 1.2 \\ \text { Questionnaire } & & 12 \text { Aug2011 }\end{array}$




\section{Behavior Related Media}

ID\# |_ا_ا_ا_ا_

M27. This section considers any handouts, cards, posters, videos, emails, or any other type of message that you may or may not have seen concerning malaria and malaria chemoprophylaxis medications.

These messages can be both at your home unit, or at any deployed location.

To make things simpler, these will all be lumped together and referred to simply as "messages" in the following questions.

Please complete all to the best of your ability by choosing the "single" best answer. Do not hesitate to ask for help if you do not understand.

M27A. Are messages posted in or around your unit that are related to the dangers of malaria?
0. No

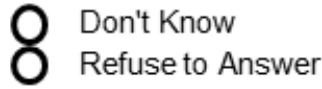

M27B. Did you find these messages informative and helpful?

(Answer "Not Applicable" if it does not apply to you)
$\begin{array}{ll}\text { O } & \text { No } \\ \text { 1. Yes }\end{array}$
Not Applicable, I never observed these messages
Refuse to Answer

M27C. Are messages posted in or around your unit that are related to the importance of properly taking your malaria chemoprophylaxis medications?
0. No
Don't Know
1. Yes
O. Refuse to Answer

M27D. Did you find these messages informative and helpful? (Answer "Not Applicable" if it does not apply to you)
○ $\begin{array}{ll}0 . & \text { No } \\ \text { 1. Yes }\end{array}$
Not Applicable, I never observed these messages
Ǒ Refuse to Answer

M27E. Have you ever paid attention to the messages posted in or around your unit related to malaria and/or malaria chemoprophylaxis medications?
0. No
O Not Applicable, I never observed these messages
O. Yes
Refuse to Answer

$\begin{array}{lll}\text { RV340 } & 23 & \text { Version } 1.2 \\ \text { Questionnaire } & & 12 \text { Aug2011 }\end{array}$




\section{Command Support}

M28. This section considers any level of command support you may or may not have concerning malaria chemoprophylaxis medications.

Please complete all to the best of your ability by choosing the "single" best answer. Do not hesitate to ask for help if you do not understand.

M28A. Is there command support at your "current" unit that reinforces that all service members take their malaria chemoprophylaxis medications as directed?
0. No
Don't Know
○ 1. Yes
Refuse to Answer

M28A1. How often can you recall your Company, Battalion, or Brigade/Group reinforcing the necessity for you to take your malaria chemoprophylaxis medications as directed?
O. Never
2. A few times
1. Once
Oे $\begin{aligned} & \text { 3. Many times } \\ & \text { Refuse to Answer }\end{aligned}$

M28B. Does your Company, Battalion, Group, Brigade ever threaten any punishment for contracting malaria after failing to take your malaria chemoprophylaxis medications as directed?
0. No
Don't Know
1. Yes
Refuse to Answer

M28B1. If so: How often has this punishment actually been enforced?

(Answer "Not Applicable" if it does not apply to you)
O. Never
1. Once
Don't Know
2. A few times
Not Applicable, this is never threatened
Ǒ 3. Many times
Refuse to Answer

M28C. How often does your Company, Battalion, Group, or Brigade recommend you discuss the importance of taking your malaria chemoprophylaxis medications with your friends and coworkers?
O. Never
2. A few times
O 1. Once
3. Many times
Refuse to Answer

M28D. Does your Company, Battalion, Group, or Brigade require that malaria chemoprophylaxis medications be included in your mission planning?
O. No
Don't Know
1. Yes
Refuse to Answer

\begin{tabular}{lll}
\hline RV340 & 24 & Version 1.2 \\
Questionnaire & & 12 Aug2011
\end{tabular}


ID\# |_ا_ا_Lا_L

M28E. How often does your Company, Battalion, or Brigade include the importance of taking your medications as directed in the unit safety briefing?
O 0. Never
O 2. A few times
O 1. Once
Q 3. Many times
O Refuse to Answer

M28F. Do your Commanders "require" that malaria chemoprophylaxis medications be addressed in the normal day-to-day operations of your unit?
O 0. No
Q Don't Know
O 1. Yes
Refuse to Answer

M28F1. If so: How often do you recall this being required?
O 0. Never
O 2. A few times
O 1. Once
O $\begin{aligned} & \text { 3. Many times } \\ & \text { Refuse to Answer }\end{aligned}$ 


\section{General Knowledge of Condom Use}

Note: The term Sexually Transmitted Disease, also referred to as an STD, is now more commonly referred to as a Sexually Transmitted Infection. Throughout this section of the questionnaire, Sexually Transmitted Infection will often be abbreviated as STI.

Additionally: Many of these questions may not relate well to practices in long-term and strictly monogamous relationships, where condom use may not be regularly practiced. If you happen to be in one of these relationships and do not regularly use a condom, please feel free to choose the Not Applicable option when provided.

Otherwise, choose the answer that best describes how condom use relates to you.

C29. The next set of questions considers your knowledge regarding condoms.

For each question, please provide the "single" best answer.

If you don't know the answer to the question, it is alright to say that you don't know.

You may also refuse to answer the question.

Do not hesitate to ask for help if you do not understand.

C29A. Have you ever used a condom?
O 0. No
O Don't Know
O 1. Yes
Refuse to Answer

C29B. Can a person get Sexually Transmitted Infections (STIs) from improper use of condoms?
○ $\begin{array}{ll}0 . & \text { No } \\ \text { 1. Yes }\end{array}$
O Don't Know
Refuse to Answer

C29C. Can a person get some of the same STIs from blood transfusions?
O 0. No
Don't Know
1. Yes
Refuse to Answer

C29D. Which is "not" an example of an STI? (Please choose only one best answer)

O 1. Gonorrhea

O 2. HIV (Human Immunodeficiency Virus)

Oे 4. Malaria

3. Chlamydia

Refuse to Answer

C29E. Can STIs cause death?
O 0 . No
Don't Know
O 1. Yes
Refuse to Answer

C29F. Have you ever been given a condom to prevent STIs?
O 0 . No
O 1. Yes
Q Don't Know
O Refuse to Answer

$\begin{array}{lrr}\text { RV340 } & 26 & \text { Version } 1.2 \\ \text { Questionnaire } & & 12 \text { Aug2011 }\end{array}$


C29G. How do condoms help to prevent Sexually Transmitted Infections (STIs)?

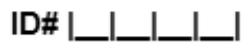
1. By removing infectious organisms
O 2. By preventing organisms from
4. By creating immunity to STIS contacting your tissue
3. By curing infections your partner
Don't Know
Refuse to Answer my have

$\mathrm{C} 29 \mathrm{H}$. Which type of condom should "not" be used for protection against STIs?
1. Colored, Latex, properly fitted
2. Ribbed, Latex, properly fitted
3. Edible, non-Latex, properly fitted
Q
4. Textured, Latex, properly fitted
Don't Know
Refuse to Answer

C29I. Are there different ways to prevent STIs, other than using condoms?
O $\begin{array}{ll}\text { 0. No } \\ \text { 1. Yes }\end{array}$
Don't Know
O Refuse to Answer

C29J. Outside a strictly monogamous relationship: Is it recommended to use a condom only every once in a while?
$\begin{array}{lll}\text { O } & \text { No } \\ \text { 1. Yes }\end{array}$
Don't Know
Refuse to Answer

C29K. Which is the most effective means of preventing Sexually Transmitted Infections (STls)?
1. There is no effective means of preventing STIS
O 4. Having unprotected intercourse with only 1 partner
. Occasional condom use
O. Not having any oral, anal, or vaginal intercourse

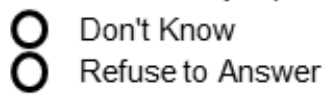

C29L. Can the military punish you for not using a condom during an act of intercourse?
O. No
Don't Know
1. Yes
Refuse to Answer

C29L2. If you believe there are any punishments, or repercussions: Can you please choose which of the following punishments or repercussions would be the most likely to occur? Please choose only one answer.

1. Article 15 , with reduction in rank

2. Article 15 , with loss of some pay

3. Court martial proceedings

4. Some type of limited punishment, such as extra duty

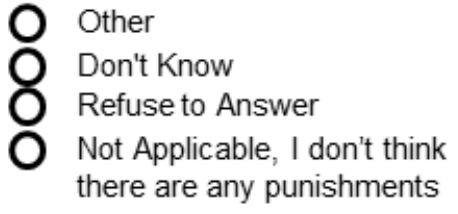




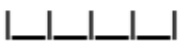

C29M. In the past 24 to 36 months: Have you yourself had any type of sexual intercourse?
O 0. No
O Don't Know
O 1. Yes
O Refuse to Answer

C29N. Does sexual intercourse include oral, anal, and vaginal intercourse?
O 0 . No
O Don't Know
O 1. Yes
Refuse to Answer

C29N1. Which is "not" a form of sexual intercourse? (Please select only one answer)
O 1. Anal
O 4. Self Masturbation
O 2. Vaginal
Don't Know
O 3. Oral
Refuse to Answer

C29O. How many times have you had sexual intercourse in the past $24-36$ months, with or without a condom?
O 1. $1-5$ times
6. 61 to 100 times
O 2. 6-10 times
3. 11 to 20 times
O 7. More than 100 times
O. 21 to 40 times
Don't Know
O 5. 41 to 60 times
O Refuse to Answer
Not Applicable- I have not had any sexual intercourse in this period

C29P. Have you yourself known of people being infected with "any"

Sexually Transmitted Infection (STI)?
O 0. No
O Don't Know
O 1. Yes
O Refuse to Answer

C29Q. Can you become ill, or show signs of infection from an STI long after you have had any type of sexual intercourse?
O 0 . No
O Don't Know
O 1. Yes
Refuse to Answer

$\begin{array}{lrr}\text { RV340 } & 28 & \text { Version } 1.2 \\ \text { Questionnaire } & 28 \text { Aug2011 }\end{array}$


C30. The next set of questions considers your general attitudes, opinions, and ideas about condom use.

Please answer each question to the best of your ability with the "single" best answer. Do not hesitate to ask for help if you do not understand.

C30A. In your opinion: When you were given a condom, were you well informed on "how" to use it?
O 0 . No
Not Applicable, I was never given a condom
1. Yes
O Refuse to Answer
O Don't Know

C30B. In your opinion: When you were given a condom, were you well informed on "why" it was important to use it during sexual intercourse?
O 0 . No
Not Applicable, I was never given a condom
O 1. Yes
O Refuse to Answer
Ǒ Don't Know

C30C. Does it bother you to have to use a condom, even if your sex partner appears healthy?
O 0 . No
O 1. Yes
Q Don't Know
O Refuse to Answer

C30C1. If yes: How much does it bother you? (Answer "Not Applicable" if it does not apply to you)
O 1. Not much
Not Applicable, I am not bothered by condom use
2. Moderately
O Refuse to Answer
O 3. Very much

C30D. Does it bother you to "ever" have to use a condom?
O 0 . No
O Don't Know
O 1. Yes
Refuse to Answer

C30D1. If yes: How much does it bother you? (Answer "Not Applicable" if it does not apply to you)
1. Not much
O Not Applicable
O 2. Moderately
O Refuse to Answer
O 3. Very much 
C30E. Do you yourself think there are any "unpleasant" issues related to condom use that may cause you "not" to use them during intercourse?
0. No
Don't Know
1. Yes
Refuse to Answer

C30E1. If yes: How unpleasant are they? (Answer "Not Applicable" if it does not apply to you)
1. Not much
Not Applicable, no unpleasant issues
2. Moderately
Refuse to Answer
Ǒ 3. Very much

C30E2. Can you name the issues you may have? (Answer "Not Applicable" if it does not apply to you)

8

0 . No, I cannot name them

1. Yes- If you choose yes, please write your response here on the space provided:
Don't Know

Not Applicable, I have no issues

Refuse to Answer

C30F. Outside monogamous and long-term relationships: Do you think using condoms during "every sexual contact" is a hassle?
0. No
1. Yes
Not Applicable
O Don't Know
Refuse to Answer

C30F1. If yes: How much of a hassle is it? (Answer "Not Applicable" if it does not apply to you)
1. Not much
Not Applicable
2. Moderately
Refuse to Answer
3. Very much

C30G. Do you think about any punishments or other repercussions that may occur through the military if you do "not" use a condom regularly?
0. No
Don't Know
1. Yes
Ǒ Refuse to Answer

C30G1. If yes: How bad do you think those punishments or repercussions will be? (Answer "Not Applicable" if it does not apply to you)
1. Mild
Not Applicable, I don't think of any repercussions
Ǒ 2. Moderate
Refuse to Answer 
$\mathrm{C} 30 \mathrm{H}$. Do those punishments or repercussions influence you to use condoms?

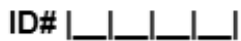
$\begin{array}{ll}\text { O } & \text { 0. No } \\ \text { 1. Yes } & \text { Pon't Know }\end{array}$
Not Applicable, I don't think of any repercussions
Refuse to Answer

$\mathrm{C} 30 \mathrm{H} 1$. If yes: How much influence do they have? (Answer "Not Applicable" if it does not apply to you)

1. Not much
2. Moderately
3. Very much

C30I. Do you "truly" believe condoms can help prevent Sexually Transmitted Infections (STIs)?
0. No
Don't Know
1. Yes
Refuse to Answer

C30J. Do you feel that the advice given by medical personnel concerning the threat of STls is "always" accurate?
0. No
Don't Know
1. Yes
Refuse to Answer

C30K. Do you sometimes think you can determine the risk of an STI better than medical personnel, particularly when your potential partner does not appear to be ill or infected?
O. No
Don't Know
1. Yes
Refuse to Answer

C30L. When deployed to a combat area: I "do not" believe considering the use of a condom to protect me from STIs is very important compared to the high priority tasks associated with combat.

O 1. Strongly Disagree

Not Applicable, I have not been to a combat area

O 2. Disagree

O Refuse to Answer

O 3. Agree

O 4. Strongly Agree

\begin{tabular}{lll}
\hline RV340 & 31 & Version 1.2 \\
Questionnaire & & 12 Aug2011
\end{tabular}




\section{Descriptive Norms}

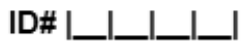

\section{C31. The next set of questions considers "your" attitudes, opinions, and ideas about how "others" behave and think about condom use.}

Please answer each question to the best of your ability with the "single" best answer. Do not hesitate to ask for help if you do not understand.

C31A. When having sexual intercourse: How many of your friends or coworkers do you believe use a condom every time?
(1. None
O 4. All
O 2. Some
Refuse to Answer
3. Most

C31B. Except in long-term and strictly monogamous relationships: How many of your friends or coworkers do you believe "do not" use condoms every time they have sexual intercourse?
1. None
4. All
2. Some
Refuse to Answer
3. Most

C31C. Except in long-term and strictly monogamous relationships: How many of your friends or coworkers do you believe feel they are actually at risk of catching a Sexually Transmitted Infection (STI) during sexual intercourse?
1. None
4. All
O 2. Some
Refuse to Answer
3. Most

C31D. How many of your friends or coworkers do you believe think that STIs are diseases that only "other" people will get, because they are too healthy to catch them?
1. None
2. All
2. Some
Refuse to Answer
Ō. Most

C31E. How many of your friends or coworkers do you think feel that STIs are not a serious issue?
1. None
4. All
O 2. Some
Refuse to Answer 
C31F. When in a new sexual relationship: How many of your friends or coworkers do you believe think about STIs on a "regular" (daily or weekly) basis?
O 1. None
O 4. All
Oे 2. Some
O Refuse to Answer

C31G. When searching for a new sexual partner: How many of your friends or coworkers do you believe plan to have a condom readily available in case the opportunity for intercourse arises?
O 1. None
O 4. All
Q 2. Some
Refuse to Answer

$\mathrm{C} 31 \mathrm{H}$. In your opinion: How many of your friends or coworkers believe the information given by medical personnel concerning the threat of STIs is "always" accurate?
O 1. None
O 4. All
Q 2. Some
O Refuse to Answer
O 3. Most

C311. In your opinion: How many of your friends or coworkers believe the information given by medical personnel concerning the threat of STIs is "not" always accurate?
O 1. None
O $\begin{aligned} & \text { 4. All } \\ & \text { Refuse to Answer }\end{aligned}$
Oे 2. Some

RV340 33




\section{Injunctive Norms}

C32. The next set of questions considers your attitudes, opinions, and ideas about how "others" approve of what "you" do concerning the use of condoms.

Note: "Outside a strictly monogamous relationship" refers to any intercourse you may have had outside a marriage or steady relationship that you considered monogamous.

Monogamous refers to intercourse with only one person during the course of a relationship.

Please answer each question to the best of your ability with the "single" best answer by indicating whether you strongly disagree, disagree, agree, or strongly agree with each statement or question. Do not hesitate to ask for help if you do not understand.

C32A. If I was having sexual intercourse outside a strictly monogamous relationship, my friends and coworkers would think that I "should" use a condom each time.
1. Strongly Disagree
4. Strongly Agree
2. Disagree
Refuse to Answer
3. Agree

C32B. Outside a strictly monogamous relationship: My friends and coworkers would think it's "OK" for me to use a condom every-so-often during sexual intercourse, instead of each time.
1. Strongly Disagree
Q 4. Strongly Agree
2. Disagree
Ǒ. Agree

C32C. Outside a strictly monogamous relationship: My friends and coworkers would think it was "OK" if I went on a "singles vacation" and "did not" take a supply of condoms with me.
1. Strongly Disagree
4. Strongly Agree
2. Disagree
Orefuse to Answer
3. Agree

C32D. Outside a strictly monogamous relationship:

My friends and coworkers think it's "OK" if I forget to use a condom.
O 1. Strongly Disagree
O 4. Strongly Agree
O 2. Disagree
Orefuse to Answer
3. Agree

\begin{tabular}{lrr}
\hline RV340 & 34 & Version 1.2 \\
Questionnaire & & 12 Aug2011
\end{tabular}




\section{Remember: Many of these questions refer to sexual intercourse "outside" strictly monogamous relationships where condom use may not be regularly practiced.}

C32E. Outside a strictly monogamous relationship: My friends and coworkers would think it's "cool" if I decided not to use a condom during sexual intercourse
1. Strongly Disagree
4. Strongly Agree
2. Disagree
Refuse to Answer
3. Agree

C32F. My friends and coworkers would think it was "OK" for me to suggest there was no threat of catching a Sexually Transmitted Infection (STI) because I did not see any evidence of them: despite what medical personnel were saying.
1. Strongly Disagree
4. Strongly Agree
2. Disagree
Refuse to Answer
Ő. Agree

C32G. My friends and coworkers would think it's "OK" if I lied about using a condom: even though I really did not use one
1. Strongly Disagree
4. Strongly Agree
2. Disagree
Orefuse to Answer
3. Agree

$\mathrm{C} 32 \mathrm{H}$. My friends and coworkers would think it's "OK" if I recommended someone use a condom, even though that person "did not" want to use one
1. Strongly Disagree
4. Strongly Agree
2. Disagree
O Refuse to Answer
Ǒ 3. Agree

C32l. My friends and coworkers would think it's "OK" for me to tell someone they "did not" have to use a condom if they "did not" want to use one, despite medical advice.
1. Strongly Disagree
4. Strongly Agree
2. Disagree
O Refuse to Answer
3. Agree

C32J. If I was a leader, my friends and coworkers would think I should set the example for others to follow, particularly using a condom during sexual intercourse outside a long-term and strictly monogamous relationship.
1. Strongly Disagree
4. Strongly Agree
O 2. Disagree
Refuse to Answer
3. Agree

$\begin{array}{lll}\text { RV340 } & 35 & \text { Version 1.2 } \\ \text { Questionnaire } & & 12 \text { Aug2011 }\end{array}$


$\mathrm{C} 32 \mathrm{~K}$. If I was a leader, others would think negatively about my character if they knew I was "not" using a condom during sexual intercourse that was outside a long-term and strictly monogamous relationship.
O 1. Strongly Disagree
Q 4. Strongly Agree
O. 2. Disagree
O็. Agree
Refuse to Answer

C32L. If I was a leader, I would not want others to know that I was "not" using a condom because it might influence them "not" to use a condom as well.
O 1. Strongly Disagree
Q 4. Strongly Agree
O 2. Disagree
Refuse to Answer
O็. Agree

C32M. My friends and coworkers would "not" approve of my actions if I caught a Sexually Transmitted Infection (STI) because I did "not" use a condom.
1. Strongly Disagree
Q 4. Strongly Agree
2. Disagree
Refuse to Answer
3. Agree

C32N. My friends and coworkers would "not" approve of my actions if I caught an STI and "compromised a mission" because I did "not" use a condom.
O 1. Strongly Disagree
Q 4. Strongly Agree
Q 2. Disagree
Refuse to Answer
Oิ. Agree

\begin{tabular}{lll}
\hline RV340 & 36 & Version 1.2 \\
Questionnaire & & 12 Aug2011
\end{tabular}


C33. You will now be asked to answer some questions about how you perceive the risk associated with Sexually Transmitted Infections (STls) and what you feel is the likelihood that you might get infected.

You may either be asked a question, or required to respond to a statement.

Please complete all to the best of your ability by choosing the "single" best answer. Do not hesitate to ask for help if you do not understand.

C33A. Do you believe Sexually Transmitted Infections (STIs) are a serious health threat?
O 0 . No
O Don't Know
O 1. Yes
O Refuse to Answer

C33B. Outside a strictly monogamous relationship: What is the likelihood that your friends and coworkers will ever contract an STI?

O 1. Not at all likely

O 2. Somewhat likely

O Not Applicable, they do not have sex outside a monogamous relationship

O 3 . Very likely

O Refuse to Answer

C33C. What is the likelihood you will ever contract an STI?

O 1. Not at all likely

O 2. Somewhat likely

O 3 . Very likely O Refuse to Answer

O Not Applicable, I am in a long-term and strictly monogamous relationship

C33D. Do you feel that STls are infections that only others will contract?
O 0 . No
O Don't Know
O 1. Yes
O Refuse to Answer

C33E. Outside a strictly monogamous relationship: When you might be dating and having sex, do you think about Sexually Transmitted Infections (STIs) on a regular (daily or weekly) basis?
O 0 . No
O 1. Yes
Q Don't Know
Not Applicable, I am in a long-term and strictly monogamous relationship
O Refuse to Answer

C33F. If you were to see individuals around you who have contracted any STIs: Do you think that would influence you to use a condom during sexual intercourse, outside a long-term and strictly monogamous relationship?
O 0 . No
O Don't Know
O 1. Yes
Refuse to Answer

\begin{tabular}{lrr}
\hline RV340 & 37 & Version 1.2 \\
Questionnaire & & 12 Aug 2011
\end{tabular}


Behavioral Outcome Related Practices

ID\# |_ا_ا_|

C34. This section of statements and questions considers the practices that may affect your use of condoms outside of obvious long-term and monogamous relationships.

You may either be asked a question, or required to respond to a statement. Please complete all to the best of your ability by choosing the "single" best answer. Do not hesitate to ask for help if you do not understand.

Remember: "Outside a strictly monogamous relationship" refers to any intercourse you may have had outside a marriage or steady relationship that you considered monogamous. Monogamous refers to intercourse with only one person during the course of a relationship.

C34A. Outside a long-term and strictly monogamous relationship: Did you yourself use a condom during every act of sexual intercourse?
( 0 . No
1. Yes
Not Applicable, Only in a monogamous relationship
Don't Know
Refuse to Answer

C34B. Outside a long-term and monogamous relationship: How often do you use a condom?
O 0 . None of the time, never
1. Some of the time, about $25 \%$ of the time
Don't Know
2. Most of the time, about $50 \%$ to $75 \%$ of the time
O 3. All the time, $100 \%$ of the time, without exception
Not Applicable, Only in a monogamous relationship
O Refuse to Answer

C34C. Outside a long-term and strictly monogamous relationship:

Did you yourself use a condom during your "last" act of sexual intercourse.
O 0 . No
Not Applicable, Only in a monogamous relationship
1. Yes
Refuse to Answer
Don't Know

C34D. I only use a condom because I fear the "repercussions" that the military may bring upon me if I catch an STI because I did not use one.
8
0 . False (No)
Not Applicable, Only in a monogamous relationship
1. True (Yes)
O Refuse to Answer

C34E. I was not using condoms at first, but when I noticed individuals around me contracting STIs: I began to use condoms during every sexual encounter.
O. False (No)
Not Applicable, This never happened to me
O. True (Yes)
Refuse to Answer
Ō Don't Know

$\begin{array}{lll}\text { RV340 } & 38 & \text { Version } 1.2 \\ \text { Questionnaire } & & 12 \text { Aug2011 }\end{array}$


ID\# |_ا_ا_ا_ |

C34F. If you did begin using condoms because of others contracting STIs: Did you use the condoms even though you had unpleasant issues associated with them?

(Answer "Not Applicable" if it does not apply to you)
O 0 . No
O Not Applicable, This never happened to me
O 1. Yes
O Refuse to Answer
O Don't Know

C34G. When I am reminded, I use condoms. Otherwise, I often forget to use them.
O 0 . No
O Not Applicable, Only in a monogamous relationship
O 1. Yes
O Refuse to Answer
O Don't Know

$\mathrm{C} 34 \mathrm{H}$. When intercourse is a possibility: I plan condoms into my missions and deployments.
O 0 . None of the time, never
O Not Applicable, intercourse was never
O 1. Some of the time, about $25 \%$ of the time a possibility during my mission planning
O 2. Most of the time, about $50 \%$ to $75 \%$ of the time
O 3. All the time, $100 \%$ of the time,
O Don't Know
O Refuse to Answer

C34l. Do you plan to use a condom the next time you have sexual intercourse outside a strictly monogamous relationship?
O 0 . No
O Not Applicable
O 1. Yes
O Refuse to Answer
O Don't Know

\begin{tabular}{lll}
\hline RV340 & 39 & Version 1.2 \\
Questionnaire & & 12 Aug2011
\end{tabular}


Interpersonal Communication

ID\# |_ا_ا_ا_|

\section{C35. This section considers communications you may have with others, that may affect your use of condoms during sexual intercourse.}

Please complete all to the best of your ability by choosing the "single" best answer. Do not hesitate to ask for help if you do not understand.

C35A. I had discussions with my friends and coworkers about the need to use condoms during sexual intercourse.
O. Never
2. A few times
O 1. Once
3. Many times

C35B. I had discussions with my friends and coworkers about the decision "not" to use condoms during sexual intercourse.
O. Never
2. A few times
O 1. Once
3. Many times
Ǒ Refuse to Answer

C35C. I advised my friends or coworkers "to use" condoms during sexual intercourse.
O. Never
2. A few times
3. Many times
Refuse to Answer

C35D. I advised my friends or coworkers "not" to use condoms during sexual intercourse.
O. Never
2. A few times
O 1. Once
3. Many times
Ǒ Refuse to Answer

C35E. I discussed the threat of sexually transmitted infections with my friends and coworkers.
O. Never
2. A few times
1. Once
O. 3any times

C35F. I was influenced "to use" condoms during sexual intercourse because of my conversations with my friends and coworkers.
0. Never
3. Many times
О̊ 1. Once
O Not Applicable, I nev

C35G. I was influenced "not to use" condoms during sexual intercourse because of my conversations with my friends and coworkers.

$\begin{array}{ll}\text { O. Never } & \text { O } \\ \text { 1. Once } & \text { 2 few times }\end{array}$
3. Many times

Not Applicable, I never had these conversations

Refuse to Answer

\begin{tabular}{lll}
\hline RV340 & 40 & Version 1.2 \\
Questionnaire & 42 12Aug2011
\end{tabular}


C36. This section considers education you may or may not have had concerning Sexually Transmitted Infections (STIs) and Condom use.

Please complete all to the best of your ability by choosing the "single" best answer. Do not hesitate to ask for help if you do not understand.

C36A. Have you "ever" had any education concerning the dangers of Sexually Transmitted Infections (STIs)?
Oे 1. Never

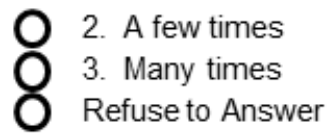

C36B. If you have had this education: Do you feel you were well informed because of it? (Answer "Not Applicable" if it does not apply to you)
$\begin{array}{ll}\text { O } & \text { No } \\ \text { 1. Yes }\end{array}$
Not Applicable, never offered to me
Refuse to Answer

C36C. Have you "ever" had any education concerning the importance of regular condom use?
O. Never
2. A few times
O 1. Once

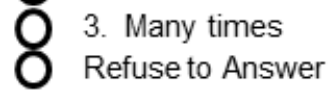

C36D. If you have had this education: Do you feel you are well informed because of it? (Answer "Not Applicable" if it does not apply to you)
$\begin{array}{ll}\text { O } & \text { No } \\ \text { 1. Yes }\end{array}$
Not Applicable, never offered to me
Refuse to Answer

C36E. Is the education on the dangers of Sexually Transmitted Infections (STIs) offered at your "current" unit?
O. No
1. Yes
Q Don't Know
Refuse to Answer

C36F. Do you feel this education informed you well? (Answer "Not Applicable" if it does not apply to you)
O 0. No
O Not Applicable, never taken at my unit
O 1. Yes
Ohefuse to Answer 
C36G. Is education on the importance of regular condom use offered at your "current" unit?
$\begin{array}{ll}\text { Oे } & 0 . \text { No } \\ \text { 1. Yes }\end{array}$
Q Don't Know
Refuse to Answer

$\mathrm{C} 36 \mathrm{H}$. Do you feel this education informed you well?

(Answer "Not Applicable" if it does not apply to you)
$\begin{array}{lll}\text { O } & \text { 0. No } \\ \text { O. Yes }\end{array}$
Not Applicable, never taken at my unit
Refuse to Answer

\section{Behavior Related Training}

C37. This section considers training you may or may not have had concerning condom use and sexually transmitted infections.

Please complete all to the best of your ability by choosing the "single" best answer. Do not hesitate to ask for help if you do not understand.

C37A. Have you ever had any training that demonstrated how to properly use a condom?
O̊ 0. Never
O 2. A few times
O 3. Many times
O Refuse to Answer

C37B. Do you feel this training prepared you well? (Answer "Not Applicable" if it does not apply to you)
O 0 . No
O 1. Yes
O Not Applicable, never offered to me
O Refuse to Answer

C37C. Is training available at your "current" unit that demonstrates how to properly use a condom?
O 0 . No
O Don't Know
O 1. Yes
O Refuse to Answer

C37D. Do you feel this training prepared you well? (Answer "Not Applicable" if it does not apply to you)
O. … №
O Not Applicable, never taken at my unit
O 1. Yes
O Refuse to Answer

\begin{tabular}{lll}
\hline RV340 & 42 & Version 1.2 \\
Questionnaire & & 12 Aug2011
\end{tabular}




\section{Behavior Related Media}

ID\# |_ا_L_ا_L

C38. This section considers any handouts, cards, posters, videos, emails, or any other type of message that you may or may not have seen concerning condom use and Sexually Transmitted Infections (STls).

These messages can be both at your home unit, or at any deployed location.

To make things simpler, these will all be lumped together and referred to simply as "messages" in the following questions.

Please complete all to the best of your ability by choosing the "single" best answer. Do not hesitate to ask for help if you do not understand.

C38A. Are messages posted in or around your unit that are related to the dangers of STIs?
O 0 . No
O Don't Know
O 1. Yes
Refuse to Answer

C38B. Did you find these messages informative and helpful?

(Answer "Not Applicable" if it does not apply to you)
O 0 . No
O Not Applicable, I never observed these messages
O 1. Yes
O Refuse to Answer

C38C. Are messages posted in or around your unit that are related to the importance of regular condom use?
O 0 . No
Q Don't Know
O 1. Yes
Refuse to Answer

C38D. Did you find these messages informative and helpful?

(Answer "Not Applicable" if it does not apply to you)
O 0 . No
O Not Applicable, I never observed these messages
O 1. Yes
O Refuse to Answer

C38E. Have you ever paid attention to the messages posted in or around your unit concerning condom use or Sexually Transmitted Infections (STIs)?
O $\begin{array}{ll}\text { 0. No } \\ \text { 1. Yes }\end{array}$
Not Applicable, I never observed these messages
Refuse to Answer

$\begin{array}{lll}\text { RV340 } & 43 & \text { Version } 1.2 \\ \text { Questionnaire } & 42 \text { Aug2011 }\end{array}$




\section{Command Support}

C39. This section considers the level of command support you may or may not have concerning condom use.

Please complete all to the best of your ability by choosing the "single" best answer. Do not hesitate to ask for help if you do not understand.

C39A. Is there command support at your "current" unit which reinforces that all service members, outside long-term and obviously monogamous relationships, should regularly use condoms?
O 0 . No
O Don't Know
O 1. Yes
O Refuse to Answer

C39A1. How often can you recall your Company, Battalion, or Brigade/Group reinforcing the necessity to use a condom to prevent STIs?
Oे $\begin{array}{ll}\text { O. Never } \\ \text { 1. Once }\end{array}$
O 2. A few times
O 3. Many times
O Refuse to Answer

C39B. Outside any long-term and obviously monogamous relationships: Does your Company, Battalion, Group, or Brigade ever threaten any punishment for catching a

Sexually Transmitted Infection (STI) after failing to use a condom during sexual intercourse?
O 0 . No
O Don't Know
O 1. Yes
Refuse to Answer

C39B1. If so, how often has this punishment actually been enforced?

(Answer "Not Applicable" if it does not apply to you)
O 0. Never
O 1. Once
O Don't Know
O. 2. A few times
Not Applicable, this is never threatened
O 3. Many times
O Refuse to Answer

C39C. How often does your Company, Battalion, Group, or Brigade recommend you discuss the importance of regular condom use with your friends and coworkers?
O 0. Never
O 2. A few times
O 1. Once
3. Many times
O Refuse to Answer

\begin{tabular}{lll}
\hline RV340 & \multirow{2}{*}{44} & Version 1.2 \\
Questionnaire & & 12 Aug2 2011
\end{tabular}


ID\# |_ا_ا_ا_ا_L

C39D. Does your Company, Battalion, or Brigade require that condoms be included in your mission planning?
O 0 . No
O Don't Know
O 1. Yes
O Refuse to Answer

C39E. How often does your Company, Battalion, or Brigade include condom use in the unit safety briefing?
O 0. Never
O 2. A few times
O 1. Once
O $\begin{aligned} & \text { 3. Many times } \\ & \text { Refuse to Answer }\end{aligned}$

C39F. Do your Commanders "require" that regular condom use be addressed in the normal day-to-day operations of your unit.
O 0 . No
Q Don't Know
O 1. Yes
O Refuse to Answer

C39F1. If so, how often do you recall this being required?
O $\begin{array}{ll}0 . & \text { Never } \\ \text { 1. Once }\end{array}$
2. A few times
O. 3. Many times
O Refuse to Answer

\begin{tabular}{lll}
\hline RV340 & Version 1.2 \\
Questionnaire & 45 & 12 Aug2011
\end{tabular}




\section{General Knowledge of Weapon Safety}

Note: Weapon Safety can be a very relative term: it can have varying degrees of meaning to different groups. An extreme example of this may be that a safe weapon to a normal suburban housewife is one that is unloaded, also has a trigger lock, and is also locked up in a gun safe.

To Special Operations members, weapon safety may have a different meaning. For example, you often carry loaded automatic weapons that may be at the ready position. Therefore, your meaning of "safe" can be different than that of the suburban housewife's. Safe, to you, may mean that the risk of someone getting shot accidentally is minimized to the greatest extent possible, or that no weapon is discharged unintentionally.

Your concept of what a "normal" level of safety may be, as a Special Operations member, is what we would like you to refer to when answering this section of the questionnaire. Also, please do not consider extreme circumstances or uncommon military scenarios where safety may have been compromised, but no one was injured.

Additionally: These questions will refer to personal and crew-served weapons only. The questions in this section are not intended to be answered in relation to Artillery, Close Air Support, or other means of indirect supporting weapons.

W40. The next set of questions considers your knowledge regarding weapon safety. For each question, please provide the "single" best answer. If you don't know the answer to the question, it is alright to say that you don't know. You may also refuse to answer the question. Do not hesitate to ask for help if you do not understand.

W40A. Are you familiar with the operation of the following weapons: M16 series rifles, M4 or M4A1 Carbine, M9 pistol, M240B Machine gun, M2 .50 Caliber Machine Gun, M249 Machine Gun, and the M203 Grenade Launcher?
O 0 . No
O Don't Know
1. Yes
O Refuse to Answer

W40A1. If you are "not" familiar with the operation of any of these weapons: Please check those you are "not" familiar with. (Please answer all that apply, or Not Applicable if you are familiar with all.)

O Not Applicable, I am familiar with the operation of all these weapons
a. M16 series rifle
O e. M2.50 Caliber Machine Gun
b. M4 or M4a1 Carbine
f. M249 Machine Gun
c. M9 Pistol
d. M240B Machine Gun
g. M203 Grenade Launcher

\section{Don't Know $\quad$ Refuse to Answer}

$\begin{array}{lrr}\text { RV340 } & 46 & \text { Version } 1.2 \\ \text { Questionnaire } & 42 \text { Aug2011 }\end{array}$


W40B. Can you be severely injured from the unsafe use of your weapon?
O 0 . No
Don't Know
1. Yes
Refuse to Answer

W40C. Can you be severely injured from another individual's unsafe use of their weapon?
O 0 . No
O Don't Know
1. Yes
O Refuse to Answer

W40D. Which is "not" a good example of weapon safety?
1. Clear all weapons at required clearing points
Don't Know
2. Provide and verify the correct ammo for all weapons
3. Ensure weapons are on safe when not being fired
4. Consider unloaded weapons as safe

W40E. Can the improper use of "any" weapon be deadly?
O 0 . No
O Don't Know
1. Yes
O Refuse to Answer

W40F. Which is "not" a correct performance step in zeroing the M4 or M4A1 Carbine?
1. Select the long-range aperture on the rear sight
Don't Know
2. Center the rear sight aperture
Refuse to Answer
O 3. Rotate the elevation knob counterclockwise to the 300 meter mark
4. Rotate the elevation knob 2 clicks counterclockwise below the 300 meter mark

W40G. Which is "not" a correct performance step in unloading the M4 or M4A1 Carbine?
1. Point the muzzle in a safe direction
2. Attempt to place the selector lever on SAFE
Don't Know
3. Observe the magazine
4. Lock the bolt open and return the charging handle forward

W40H. Which is "not" a correct performance step in the function check of an M240B Machine Gun?
1. Place safety lever to "F" position

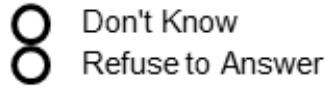
3. Ensure the cocking handle remains locked rearward
4. Place safety lever to "S" position

\begin{tabular}{lrr}
\hline RV340 & 47 & Version 1.2 \\
Questionnaire & 42 Aug2011
\end{tabular}


W40I. During Urban Operations:

ID\# |_ا_Ll_l

Is it safe to practice pointing your weapon slightly downward in the direction of travel?
O 0 . No
O Don't Know
O 1. Yes
O Refuse to Answer

W40J. During Urban Operations: Is it safe to shoot while moving faster than you can engage your targets?
O 0 . No
O Don't Know
O 1. Yes
O Refuse to Answer

W40K. During Urban Operations: Which movement technique is considered the "safest" ready position when moving in buildings or rooms?
O 1. The standing unsupported
O Don't Know
2. The low ready
O Refuse to Answer
O 3. The prone supported
O 4. The high ready

W40L. Can the military punish you for not following weapon safety guidance?
O 0 . No
O Don't Know
O 1. Yes
O Refuse to Answer

W40L2. If you believe there are any such punishments: Can you please choose which of the following punishments or repercussions would be the most likely to occur? Please choose only one.
O 1. Article 15, with reduction in rank
O 2. Article 15, with loss of some pay
O Don't Know
3. Court marital proceedings
O 4. Some type of limited punishment, such as extra duty

W40M. In the past 24 to 36 months, have you yourself had to practice weapon safety techniques during combat, live fire training, or other high risk military deployments?
O 0 . No
O Don't Know
O 1. Yes
O Refuse to Answer

W40N. If so, How long was your last high risk deployment, where you operated a weapon?
O 1. 1-7 days
O 6. Between 1 to 2 years
O. 2. $8-30$ days
O 7. More than 2 years
O. 31-90 days
O Don't Know
O 4. $91-180$ days
O 5.181 to 365 days
O Not Applicable, I was not deployed in this period
O Refuse to Answer

W40O. Have you known of people who were injured due to the unsafe operation of a weapon?
O 0 . No
O Don't Know
O 1. Yes
O Refuse to Answer

\begin{tabular}{lll}
\hline RV340 & 48 & Version 1.2 \\
Questionnaire & & 12 Aug2011
\end{tabular}




\section{General Attitudes Towards Weapon Safety ID\#I_L_LI}

\section{W41. The next set of questions consider your general attitudes, opinions, and ideas about weapon safety.}

Please answer each question to the best of your ability with the "single" best answer. Do not hesitate to ask for help if you do not understand.

W41A. In your opinion: When you were initially issued a military weapon, were you well informed on "how" to use it?
$\begin{array}{ll}\text { O̧ } & \text { No } \\ \text { 1. Yes } & \text { Don't Know }\end{array}$
Not Applicable, I was never issued a weapon
O Refuse to Answer

W41B. In your opinion: When you were issued a military weapon, were you well informed on "why" it was important to follow safely guidance?
O 0 . No
Not Applicable, I was never issued a weapon
1. Yes
O Refuse to Answer
Ǒ Don't Know

W41C. Does it bother you to have to practice weapon safety at all times?
$\begin{array}{ll}\text { O } & \text { No } \\ \text { 1. Yes }\end{array}$
Don't Know
Refuse to Answer

W41C1. If yes: How much does it bother you? (Answer "Not Applicable" if it does not apply to you)
1. Not much
Not Applicable, it does not bother me at all
2. Moderately
O Refuse to Answer
3. very much

W41D. Do you yourself think there are any "unpleasant" or "bothersome" issues related to weapon safety that may cause you "not" to practice it?
0. No
Don't Know
1. Yes
Refuse to Answer

W41D1. If yes: How unpleasant or bothersome are they? (Answer "Not Applicable" if it does not apply to you)
1. Not much
O. 2. Moderately
Not Applicable, I have no issues
3. Very much
Refuse to Answer

\begin{tabular}{lll}
\hline RV340 & Version 1.2 \\
Questionnaire & 49 & 12 Aug2011
\end{tabular}


W41D2. Can you name those issues? (Answer "Not Applicable" if it does not apply to you)

$$
\begin{aligned}
& \text { O No, I cannot define them } \quad O \text { Don't Know } \\
& \text { ○ 1. Yes- Please specify: _ـ }
\end{aligned}
$$

W41E. Do you think that following weapon safety "at all times" is a hassle?
Oे 0. No
O Don't Know
1. Yes
Refuse to Answer

W41E1. If yes: How much of a hassle is it? (Answer "Not Applicable" if it does not apply to you)
O 1. Not much
O. 2. Moderately
Not Applicable, it does not bother me at all
O 3. very much
O Refuse to Answer

W41F. Do you think about any punishments, or other repercussions that may occur through the military if you do "not" follow weapon safety guidance?
O 0 . No
O Don't Know
O 1. Yes
O Refuse to Answer

W41F1. If yes: How bad do you think those punishments or repercussions will be?

(Answer "Not Applicable" if it does not apply to you)
Oे 1. Mild
2. Moderate
3. Severe
O Not Applicable, I don't think of any repercussions
O Refuse to Answer

W41G. Do those punishments or repercussions influence you to follow weapon safety?
O 0 . No
Not Applicable, I don't think of any repercussions
O 1. Yes
Refuse to Answer

W41G1. If yes: How much influence do they have? (Answer "Not Applicable" if it does not apply to you)
O 1. Not much
O Not Applicable, I don't think of any repercussions
O 2. Moderately
O Refuse to Answer
3. Very much

$\begin{array}{llr}\text { RV340 } & 50 & \text { Version } 1.2 \\ \text { Questionnaire } & & 12 \text { Aug2011 }\end{array}$


ID\# |_ا_ا_Lا_L

W41H. Do you "truly" believe following weapon safety guidance can prevent injury or death?
O 0 . No
O Don't Know
O 1. Yes
Refuse to Answer

W41I. Do you feel that the advice given by your leadership concerning weapon safety is "always" accurate?
O 0 . No
O Don't Know
O 1. Yes
Refuse to Answer

W41J. Do you sometimes think you can determine the safety risks concerning military weapons better than what your leadership can, particularly when you are not being directly supervised?
O 0 . No
Q Don't Know
O 1. Yes
Refuse to Answer

W41K. When deployed to a combat area: I "do not" believe considering weapon safety is very important compared to the high priority tasks associated with combat.
O 1. Strongly Disagree
O Not Applicable, I have not been to a combat area
2. Disagree
O Refuse to Answer
O 3. Agree
O 4. Strongly Agree

$\begin{array}{lll}\text { RV340 } & 51 & \text { Version } 1.2 \\ \text { Questionnaire } & & 12 \text { Aug2011 }\end{array}$




\section{Descriptive Norms}

ID\# |_ا_ا_ا_

W42. The next set of questions consider "your" attitudes, opinions, and ideas about how "others" behave and think about weapon safety.

Please answer each question to the best of your ability with the "single" best answer. Do not hesitate to ask for help if you do not understand.

W42A. When operating weapons: How many of your friends or coworkers do you believe "always" follow the recommended safety guidance?
O 1. None
O 4. All
Oे 2. Some
O Refuse to Answer

W42B. How many of your friends or coworkers do you believe "do not" follow the recommended safety guidance?
O 1. None
O 4. All
O 2. Some
O Refuse to Answer
O. Most

W42C. How many of your friends or coworkers do you believe feel they are actually at risk of getting injured due to an unsafe act with a weapon?
O 1. None
O 4. All
Oூ 2. Some
O Refuse to Answer

W42D. How many of your friends or coworkers do you believe think that weapon safety accidents only happen to "other" people, because they are too experienced?
O 1. None
O 4. All
O 2. Some
O Refuse to Answer
O 3. Most

W42E. How many of your friends or coworkers do you think feel that weapon accidents are not a serious issue?
O 1. None
O 4. All
O 2. Some
Refuse to Answer 
W42F. When operating a weapon: How many of your friends or coworkers do you believe think of weapon safety on a "regular" (daily or weekly) basis?
1. None
4. All
О人 2. Some
Refuse to Answer

W42G. Prior to preparing for any sort of weapon use: How many of your friends or coworkers do you believe plan to review and consider weapon safety guidelines?
1. None
O. All
О人 2. Some
Refuse to Answer

$\mathrm{W} 42 \mathrm{H}$. In your opinion: How many of your friends or coworkers believe the weapon safety guidelines they receive from their leadership is "always" appropriate?
(1. None
4. All
Ǒ 2. Some
Refuse to Answer

W42I. In your opinion: How many of your friends or coworkers believe the weapon safety guidelines they receive from their leadership is "not" always appropriate?
1. None
O̧ 2. Some
4. All
3. Most
O Refuse to Answer

\begin{tabular}{lll}
\hline RV340 & \multirow{2}{*}{53} & Version 1.2 \\
Questionnaire & & 12 Aug2011
\end{tabular}




\section{Injunctive Norms}

W43. The next set of questions consider your attitudes, opinions, and ideas about how "others" approve of what "you" do concerning weapon safety.

Please answer each question to the best of your ability with the "single" best answer by indicating whether you strongly disagree, disagree, agree, or strongly agree with each statement or question. Do not hesitate to ask for help if you do not understand.

W43A. If I were operating a weapon, my friends and coworkers would think that I "should" use the recommended safety guidance.
O 1. Strongly Disagree
4. Strongly Agree
2. Disagree
Refuse to Answer
3. Agree

W43B. My friends and coworkers think it's "OK" for me to only use the recommended safety guidance "every once-in-a-while," instead of "all the time."

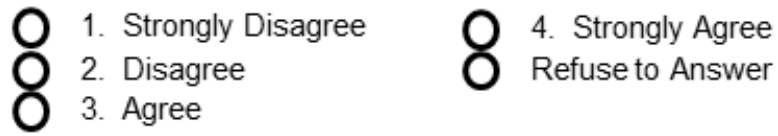

W43C. My friends and coworkers would think it was "OK" if I went on a multi-day combat or live fire mission, and did not consider weapons safety.
1. Strongly Disagree
4. Strongly Agree
2. Disagree
Refuse to Answer
Ǒ. Agree

W43D. My friends and coworkers think it's "OK" if I forget to implement the recommended safety guidance.
1. Strongly Disagree
O 4. Strongly Agree
2. Disagree
Refuse to Answer
3. Agree

W43E. My friends and coworkers would think it's "cool" if I decided not to implement the recommended weapon safety guidance during a live fire mission.
1. Strongly Disagree
Q. Strongly Agree
O 2. Disagree
Ǒ Refuse to Answer
3. Agree

\begin{tabular}{lrr}
\hline RV340 & 54 & Version 1.2 \\
Questionnaire & & 12 Aug2011
\end{tabular}


W43F. My friends and coworkers would think it was "OK" for me to suggest there was "no reason" to implement the recommended weapon safety guidance because I did not see any reason for it, despite what leadership says.
O 1. Strongly Disagree
4. Strongly Agree
O. 2. Disagree
O Refuse to Answer
O. Agree

W43G. My friends and coworkers would think it's "OK" if I lied about implementing the recommended weapon safety guidance, even though I really did not.
O 1. Strongly Disagree
O 4. Strongly Agree
Q 2. Disagree
3. Agree

W43H. My friends and coworkers would think it's "OK" if I recommended someone to implement the recommended weapon safety guidance, even though that person "did not" want to.
O 1. Strongly Disagree
O 4. Strongly Agree
O 2. Disagree
O Refuse to Answer
O. Agree

W43I. My friends and coworkers would think it's "OK" for me to tell someone they "did not" have to implement the recommended weapon safety guidance if they "did not" want to do so.
O 1. Strongly Disagree
O 4. Strongly Agree
Q 2. Disagree
O Refuse to Answer
О 3. Agree

W43J. If I were a leader: my friends and coworkers would think I should set the example for others to follow, particularly implementing the recommended weapon safety guidance.
O 1. Strongly Disagree
O 4. Strongly Agree
○े 2. Disagree
O Refuse to Answer

W43K. If I were a leader: others would think negatively about my character if they knew I was "not" implementing the recommended weapon safety guidance.
$\begin{array}{ll}\text { Oे } & \text { 1. Strongly Disa } \\ \text { 2. Disagree } \\ \text { 3. Agree }\end{array}$
O 4. Strongly Agree

$\begin{array}{lll}\text { RV340 } & 55 & \text { Version } 1.2 \\ \text { Questionnaire } & & 12 \text { Aug2011 }\end{array}$


ID\# |_ا_ا_Lا_L

W43L. If I were a leader: I would not want others to know that I was "not" implementing the recommended weapon safety guidance, because it might influence them to do the same.

O $\begin{array}{lll}\text { 1. Strongly Disagree } & \text { Q } & \text { 4. Strongly Agree } \\ \text { 2. Disagree } & \bigcirc & \text { Refuse to Answer }\end{array}$

О 3. Agree

W43M. My friends and coworkers would "not" approve of my actions if I was injured because I ignored the recommended weapon safety guidance.

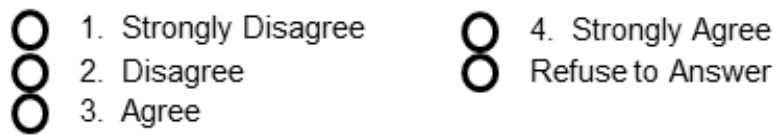

W43N. My friends and coworkers would "not" approve of my actions if I were injured and "compromised a mission" because I did "not" implement the recommended weapon safety guidance.

O 1. Strongly Disagree $\quad \mathrm{Q}$ 4. Strongly Agree

Q 2. Disagree

Refuse to Answer

3. Agree 


\section{Perceived Susceptibility and Risk}

W44. You will now be asked to answer some questions about how you perceive the risks associated with Weapon Use and the likelihood that you, or others may be injured while using them. You may either be asked a question, or be required to respond to a statement.

Please complete all to the best of your ability by choosing the "single" best answer. Do not hesitate to ask for help if you do not understand.

W44A. Do you believe weapons can cause serious injuries?
0. No
Don't Know
○ 1. Yes
Refuse to Answer

W44B. What is the likelihood that your friends and coworkers may ever be injured when operating weapons?
O 1. Not at all likely
2. Somewhat likely
Not Applicable, they do not operate any weapons
O̊ 3. Very likely
O Refuse to Answer

W44C. What is the likelihood you will ever be injured with a military weapon?

O 1. Not at all likely

2. Somewhat likely

Ǒ. Very likely

Not Applicable, I never operate a weapon

Refuse to Answer

W44D. Do you feel that injuries from the unsafe operation of weapons will only happen to others?
O. No
Don't Know
Refuse to Answer

W44E. When operating a military weapon, do you think about the possibility of injury on a regular (daily or weekly) basis?
O. No
Don't Know
1. Yes
Ǒ Not Applicable, I never operate a weapon

W44F. If you were to see individuals around you getting injured by unsafe acts: Do you think that would influence you to focus more closely on the recommended weapon safety guidance?
O. No
Don't Know
1. Yes
Refuse to Answer

\begin{tabular}{lll}
\hline RV340 & 57 & Version 1.2 \\
Questionnaire & & $12 \mathrm{Aug} 2011$
\end{tabular}


Behavioral Outcome Related Practices

ID\# |_ا_ا_ا_|

W45. This section of statements and questions consider the practices that may affect your use of the recommended weapon safety guidance. You may either be asked a question, or required to respond to a statement.

Please complete all to the best of your ability by choosing the "single" best answer. Do not hesitate to ask for help if you do not understand.

W45A. Do you yourself follow the recommended weapon safety guidance at all times?
8
0 . No
O Not Applicable, I have never used a weapon
O 1. Yes
O Refuse to Answer
Don't Know

W45B. How often do you follow weapon safety guidance? (Please choose only "one" answer)
O 0 . None of the time, never
O 1. Some of the time, about $25 \%$ of the time
O 2. Most of the time, about $50 \%$ to $75 \%$ of the time
O 3. All the time, $100 \%$ of the time, without exception
O Not Applicable, I have never used a weapon
O Refuse to Answer

W45C. I only follow the recommended weapon safety guidance because I "fear the repercussions" that the military may bring upon me if $\mathrm{I}$, or someone else is injured.
O. False (No)
Not Applicable, I have never used a weapon
Refuse to Answer
1. True (Yes)
Don't Know

W45D. I was not following the recommended weapon safety guidance at first, but when I noticed others being injured: I began to follow it very closely.
O 0 . False (No)
Not Applicable, this never happened to me
Q 1. True (Yes)
O Refuse to Answer

W45E. If you began following the safety guidance because you noticed these injuries: Did you do this
"without exception" from that point on? (Answer "Not Applicable" if it does not apply to you.)
O 0 . No
O 1. Yes
Not Applicable, this never happened to me
O Don't Know
O Refuse to Answer

$\begin{array}{lll}\text { RV340 } & 58 & \text { Version } 1.2 \\ \text { Questionnaire } & & 12 \text { Aug2011 }\end{array}$


ID\# |_ا_ا_ا_ا_L

W45F. When I am reminded, I follow the safety guidance. Otherwise, I often forget to follow it.
O 0. False (No)
O Not Applicable, I have never operated weapons
O 1. True (Yes)
O Refuse to Answer
O Don't Know

W45G. When weapons are involved: I plan weapon safety guidance into my missions

O 0 . None of the time, never

O 1. Some of the time, about $25 \%$ of the time

O 2. Most of the time, about $50 \%$ to $75 \%$ of the time

O 3. All the time, $100 \%$ of the time, without exception
O Not Applicable, I have never conducted mission planning when weapons are involved

O Don't Know

O Refuse to Answer

W45H. Do you plan to follow the recommended weapon safety guidance the next time you operate a weapon?
O 0 . No
O Not Applicable
O 1. Yes
O Refuse to Answer
O Don't Know 
Interpersonal Communication

ID\# |_ا_ا_ا_ا_

W46. This section considers communications you may have with others, that may affect you following recommended weapon safety guidance.

Please complete all to the best of your ability by choosing the "single" best answer. Do not hesitate to ask for help if you do not understand.

W46A. I discussed the need to follow the recommended weapon safety guidance with my friends and coworkers.
O $\begin{array}{ll}\text { 0. Never } \\ \text { 1. Once }\end{array}$
2. A few times
3. Many times
Refuse to Answer

W46B. I discussed the decision "not" to follow the recommended weapon safety guidance with my friends and coworkers.
O $\begin{array}{ll}0 . & \text { Never } \\ \text { 1. Once }\end{array}$
2. A few times
3. Many times
Refuse to Answer

W46C. I advised my friends or coworkers "to follow" the recommended weapon safety guidance.
O. Never
2. A few times
O 1. Once
O. 3. Many times
Ǒ Refuse to Answer

W46D. I advised my friends or coworkers "not" to follow the recommended weapon safety guidance.
O 0. Never
2. A few times
3. Many times
Ǒ Refuse to Answer

W46E. I discussed the threat of injuries related to the unsafe operation of weapons with my friends and coworkers.
O $\begin{array}{ll}\text { 0. Never } \\ \text { 1. Once }\end{array}$
2. A few times
3. Many times
Refuse to Answer

W46F. I was influenced "to follow" the recommended weapon safety guidance because of conversations with my friends and coworkers.
O. Never
3. Many times
O̊ 1. Once
O̊ Not Applicable, I never had these conversations
Refuse to Answer

W46G. I was influenced "not to follow" the recommended weapon safety guidance because of conversations with my friends and coworkers.

$\begin{array}{lll}\text { O } & \text { 0. Never } & \text { O } \\ \text { 1. Once } & \text { 2. A few times }\end{array}$

RV340

Questionnaire
O. Many times

Not Applicable, I never had these conversations

Refuse to Answer
60

12Aug2011 
W47. This section considers education you may or may not have had concerning weapon safety.

Please complete all to the best of your ability by choosing the "single" best answer. Do not hesitate to ask for help if you do not understand.

W47A. Have you "ever" had any education concerning the dangers of operating a military weapon?
Oे $\begin{array}{ll}\text { O. Never } \\ \text { 1. Once }\end{array}$
2. A few times
O. 3. Many times
Refuse to Answer

W47B. If you have had this education: Do you feel you were well informed because of it? (Answer "Not Applicable" if it does not apply to you)
$\begin{array}{ll}\text { O } & \text { 0. No } \\ \text { 1. Yes }\end{array}$
O Not Applicable, never offered to me
O Refuse to Answer

W47C. Have you "ever" had any education concerning the importance of following weapon safety guidance?
Oे $\begin{array}{ll}\text { 0. Never } \\ \text { 1. Once }\end{array}$
O 2. A few times
O 1. Once
O $\begin{aligned} & \text { 3. Many times } \\ & \text { Refuse to Answer }\end{aligned}$

W47D. If you have had this education: Do you feel you are well informed because of it? (Answer "Not Applicable" if it does not apply to you)
$\begin{array}{ll}\text { O } & \text { 0. No } \\ \text { 1. Yes }\end{array}$
O Not Applicable, never offered to me
O Refuse to Answer

W47E. Is the education on the dangers of operating a military weapon offered at your "current" unit?
O 0 . No
O Don't Know
O 1. Yes
O Refuse to Answer

W47F. Do you feel this education informed you well? (Answer "Not Applicable" if it does not apply to you)
$\begin{array}{ll}\text { Oे } & 0 . \text { No } \\ \text { 1. Yes }\end{array}$
Not Applicable, never taken at my unit
Refuse to Answer

\begin{tabular}{lll}
\hline RV340 & 61 & Version 1.2 \\
Questionnaire & & 12 Aug2011
\end{tabular}


W47G. Is education on the importance of following weapon safety guidance offered at your "current" unit?
O 0. No
Don't Know
○ 1. Yes
Ohefuse to Answer

W47H. Do you feel this education informed you well? (Answer "Not Applicable" if it does not apply to you)
0. No
1. Yes
Not Applicable, never taken at my unit
Refuse to Answer

\section{Behavior Related Training}

W48. This section considers training you may or may not have had concerning weapon safety.

Please complete all to the best of your ability by choosing the "single" best answer. Do not hesitate to ask for help if you do not understand.

W48A. Have you had training that demonstrated how to safely operate the military weapons that were issued to you?
O. Never
2. A few times
1. Once
O. Many times

W48B. Do you feel this training prepared you well? (Answer "Not Applicable" if it does not apply to you)
0. No
1. Yes
Not Applicable, never offered to me
Oh Refuse to Answer

W48C. Is training available at your "current" unit that demonstrates how to safely operate your military weapon?
0. No
Don't Know
1. Yes
Refuse to Answer

W48D. Do you feel this training prepared you well? (Answer "Not Applicable" if it does not apply to you)
$\bigcirc \begin{array}{ll}0 . & \text { No } \\ \text { 1. Yes }\end{array}$
Not Applicable, never taken at my unit 


\section{Behavior Related Media}

W49. This section considers any handouts, cards, posters, videos, emails, or any other type of message that you may or may not have seen concerning weapon safety. These messages can be both at your home unit, or at any deployed location.

To make things simpler, these will all be lumped together and referred to simply as "messages" in the following questions.

Please complete all to the best of your ability by choosing the "single" best answer. Do not hesitate to ask for help if you do not understand.

W49A. Are messages posted in or around your unit that are related to the dangers associated with the unsafe operation of weapons?
0. No
Don't Know
1. Yes
Refuse to Answer

W49B. Did you find these messages informative and helpful? (Answer "Not Applicable" if it does not apply to you)
O. No $\begin{array}{ll}\text { 1. Yes } \\ \text { 1. }\end{array}$
Not Applicable, I never observed these messages
Ǒ Refuse to Answer

W49C. Are messages posted in or around your unit that are related to the importance of following weapon safety recommendations?
O 0 . No
Don't Know
1. Yes
Refuse to Answer

W49D. Did you find these messages informative and helpful?

(Answer "Not Applicable" if it does not apply to you)
O 0 . No
O Not Applicable, I never observed these messages
1. Yes
O Refuse to Answer

W49E. Have you ever paid attention to the messages posted in or around your unit concerning the safe operation of weapons?
O. No
O Not Applicable, I never observed these messages
1. Yes
O Refuse to Answer

$\begin{array}{lll}\text { RV340 } & 63 & \text { Version } 1.2 \\ \text { Questionnaire } & & 12 \text { Aug2011 }\end{array}$




\section{Command Support}

W50. This section considers the level of command support you may or may not have concerning weapon safety.

\section{Please complete all to the best of your ability by choosing the "single" best answer. Do not} hesitate to ask for help if you do not understand.

W50A. Is there command support at your "current" unit that reinforces all service members to practice the recommended weapon safety guidelines at all times?
0. No
Don't Know
1. Yes
Refuse to Answer

W50A1. How often can you recall your Company, Battalion, or Brigade/Group reinforcing the necessity to follow the recommended weapon safety guidelines?
O. Never
2. A few times
1. Once
Oे $\begin{aligned} & \text { 3. Many times } \\ & \text { Refuse to Answer }\end{aligned}$

W50B. Does your Company, Battalion, Group, or Brigade ever threaten any punishment for "not" following the recommended weapon safety guidelines?
O 0 . No
Don't Know
1. Yes
Refuse to Answer

W50B1. If so: How often has this punishment actually been enforced?

(Answer "Not Applicable" if it does not apply to you)
O 0. Never
O Don't Know
1. Once
Not Applicable, this is never threatened
2. A few times
3. Many times
Refuse to Answer

W50C. How often does your Company, Battalion, Group, or Brigade recommend you discuss the importance of following the recommended weapon safety guidelines with your friends and coworkers?
O. Never
2. A few times
1. Once
3. Many times
Refuse to Answer

W50D. Does your Company, Battalion, or Brigade require the recommended weapon safety guidelines be included in your mission planning?

\begin{tabular}{lllll} 
& O & $\begin{array}{l}0 . \\
\text { No } \\
\text { 1. Yes }\end{array}$ & O & $\begin{array}{l}\text { Don't Know } \\
\text { Refuse to Answer }\end{array}$ \\
\hline $\begin{array}{l}\text { RV340 } \\
\text { Questionnaire }\end{array}$ & & 64 & Version 1.2 \\
12 Aug 2011
\end{tabular}


W50E. How often does your Company, Battalion, or Brigade include weapon safety in the unit safety briefing?
О $\begin{array}{ll}\text { O. } & \text { Never } \\ \text { 1. Once }\end{array}$
2. A few times
Оิ 3. Many times

W50F. Do your Commanders "require" that weapon safety be addressed in the normal day-to-day operations of your unit.
$\bigcirc \begin{array}{ll}0 . & \text { No } \\ \text { 1. Yes }\end{array}$
Don't Know
Ǒ Refuse to Answer

W50F1. If so: How often do you recall this being required?
O̊ 0. Never
2. A few times
3. Many times
Refuse to Answer

\section{Survey Admin Questions}

\section{Please answer a few administrative questions that will help improve this survey in the future.}

A51. About how much time did it take you to complete this survey?
O 1. Less than 30 minutes
2. Between 30 and 60 minutes
3. Between 60 and 90 minutes
4. Between 90 and 120 minutes
O 5. More than 120 minutes
Don't Know
Not Applicable, I did not complete the survey
Refuse to Answer

A52. Can you please tell us if any questions offended you?
O 0 . No. None offended me.
Don't Know
1. Yes. One or more questions did offend me.
O Refuse to Answer

A53. If any questions did offend you: Please tell us which ones.

\section{Thank You Very Much.}

\section{Your assistance and time have been very helpful. Have a Great Day.}

$\begin{array}{lcc}\text { RV340 } & 65 & \text { Version } 1.2 \\ \text { Questionnaire } & & 12 \text { Aug2011 }\end{array}$




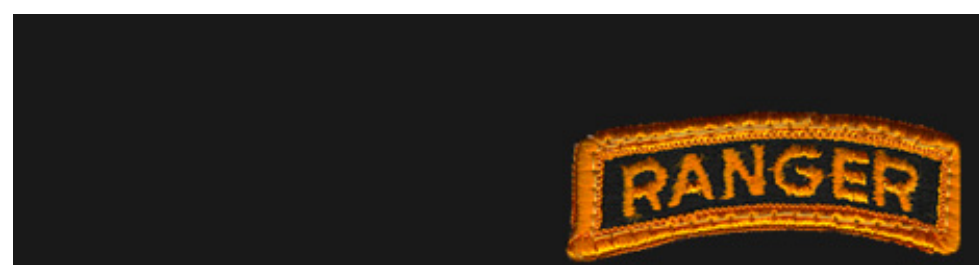


Appendix 3.2 - Key Principles for Creating a Survey Questionnaire 


\section{Key Principles for Creating a Survey Questionnaire}

In order to produce a survey instrument that would measure the appropriate objectives and withstand some level of both scientific and theoretical scrutiny, a thorough understanding of survey questionnaire design was necessary. The purpose of this paper is to provide a brief description of the key principals taken from that research.

Creating a questionnaire, particularly one that simultaneously measures three endpoints, is a complex process that requires a firm grasp of the fundamentals of questionnaire design, as well as a great deal of thought and creativity. This paper cannot relay every detail of questionnaire design philosophy, describe the in-depth thought required of the designer, or completely intimate the meticulous attention to detail needed for each question in a questionnaire; rather, it attempts to identify some of the more salient points that may be neglected or even ignored.

The information will be presented in bullet comment format and focus mainly on areas of concern related to the construction of proctored and self administered questionnaires. The bullet comments presented are a compilation of the information gathered from four sources: Asking Questions: A Practical Guide to Questionnaire Design, by Seymour Sudman and Norman M. Bradburn (1983); Survey Questions: Handcrafting the Standardized Questionnaire, by Jean M. Converse, \& Stanley Presser (1986); The Art of Asking Questions, by Stanley L. Payne (1951); and Improving Interview Method and Questionnaire Design, Norman M. Bradburn, \& Seymour Sudman (1979). The information within each of these sources overlap and are presented very similarly. Interestingly, though the time span between the earliest and latest of these references is approximately 35-years, no contrasting or significant differences were noted between them.

\section{Basic Construction}

- The shorter the questionnaire, the better; nevertheless, the instrument must capture enough data to draw sufficient conclusions. 
- No true page number and question limits exist. Some recommend limiting mail-out surveys to 12 pages. Less salient topics, especially when mailed out, may require much shorter questionnaires.

- There is no single content format, but the format choice must be well thought out, easily followed, professional in appearance, and pilot tested.

- The booklet is recommended presentation format; with heavy paper for the cover.

- The survey should appear professionally prepared in all areas.

- The first page contains the study title, organization, date, and other identifying data.

- Use sufficiently large and clear print, sufficient spacing, numbering, parallel columns, facing pages, and directing arrows when necessary.

- Use of vertical answers is recommended.

- Skip questions should be placed directly after the answer.

- Appropriate space should be provided for open ended question answers.

- Precoding of all closed end questions will facilitate data processing.

- Always end the survey with a "Thank You."

Important Considerations

- Remember to use common language with proper grammar.

- Try to capture the respondent's attention early with a subject related questions.

- Define the level of threat through the eyes of the respondent, not the researcher.

- Be courteous and never give the appearance of being "above" your respondents.

- Do your best to ensure the environment is friendly and comfortable.

- Always end the survey with a "Thank You."

General Question Design

- Be specific, and clear.

- Stay focused. Keep question topics consolidated and well organized.

- Try not to overlook any alternatives.

- Make sure "you" understand the question. Tricky questions can be tricky for you too.

- Be aware of quintamensional design which captures the five elements of opinion: awareness, general opinion, specific opinion, reasons, and intensity.

- Ask the five W's: who, what, where, when, and sometimes why.

- Trade language or jargons are ok for specific populations who are "all" familiar with them. They are not recommended for use on the general public.

- Do not begin with threatening questions. Start with the least threatening, and then gradually move to the more threatening questions.

- Ask broad questions first, and then ask those that require more refined or specific answers.

- Ask knowledge questions before asking for or measuring attitudes.

- It is better to begin with closed questions, than open questions, though a few open questions may be used early to spark interests.

- Demographic questions may be perceived as threatening and are sometimes recommended to be placed towards the end of the questionnaire. Other times they may be used as warm-up questions. No hard rule is set as to how to use demographics. Their placement must be thoroughly considered with each instrument and population.

- Demographics may be used early to qualify respondents to proceed with other questions.

- Do not ask for any more demographic questions than necessary. Asking unnecessary questions may have a derogatory effect. 
- Do not ask leading questions that may have an obvious response.

(e.g., Do you feel that voting for the orange party will stop the pink party from taking away your rights as a taxpaying citizen?)

- Avoid "double barreled" questions; those that combine more than one opinion, and may provide for more than one answer.

(e.g., Is the food tasty and do you think it will sell?)

- Avoid "one and a half barreled" questions, where a straight forward question is asked and second opinion, or object is introduced within the answer choices.

- Avoid gender specific pronouns like he, she, him, or her unless absolutely appropriate.

- Be sure both the question and the available answers are clear. Ambiguous questions and non-mutually exclusive answers can threaten the validity of the questionnaire.

- Fully restate any question that refers back to any other question.

- Having the "don't know" answer choice can have two effects:

○ (1) if the question is phrased, "as far as you know," or "do you happen to know," it may be inappropriately or over selected;

○ (2) if it is "not" given as choice in a dichotomous question, it may lead to the respondent to guessing.

- Generally, "don't know" is a good choice to have as a response because it shows the respondent that is it "ok" not to know the answer.

- Additional choices include "no opinion" or "refuse to answer"

- The middle choice is often chosen before any other, especially if guessing, or if the respondent does not have a strong opinion on the subject. In this case, it may be necessary to ask 2 questions: one that determines the opinion, and a second to determine the intensity of that opinion.

- Unipolar questions that one-sided or presence/absence of something, such as not satisfied, slightly satisfied, moderately, etc.; may be better suited for providing more specific results than Bipolar questions where the answer choice may be at either end of a spectrum (e.g., strongly agree vs. strongly disagree). Nevertheless, bipolar questions may reveal broader dimensions.

- The least socially desirable response should be at the beginning of a response scale. Respondents tend to pick the "most socially desired" response first if given the choice, especially if listed before a "lesser socially desired" response; despite the fact that the most appropriate choice for the respondent would be the "lesser desired" response.

- Open ended questions are easier to construct, and may offer great depth into a topic.

○ But, they are much more difficult to analyze.

- Closed ended questions are easy to analyze.

○ But are much more difficult to construct, and may provide limited insight.

- It is often recommended to limit verbal rating scales to have no more than 3 or 5 levels (e.g., Bad, Good, Better, etc). But this is not always the case, as some authors state, "your respondents may be able to think."

- Numerical scales are often recommended when more detail than 3 to 5 levels are required (e.g., Cold <= $12345678910=>$ Hot).

- Consider using pictures, graphs, and analogies such as thermometers or ladders.

- Avoid reliability checks. They are often considered annoying.

- Always end the survey with a "Thank You."

Knowledge Questions

- Do not ask a question that includes the answer inside it. 
(e.g., Are you aware of the Red Balloon club?) The existence of the red balloon club is obvious; therefore, any inferred knowledge is invalid.

- Do not overestimate knowledge; buy asking for specific names, places, brands, etc.

- You may compensate for guessing by asking several questions on the same topic.

- Do not ask respondents to perform complicated arithmetic calculations.

- Knowledge questions may be considered threatening. This can be reduced by wording questions like "in your opinion" and "from what you recall", etc. The "don't know" response may also help.

- If a subject is not well known, a knowledge question may actually measure attitudes. (e.g., Is perestroika a capitalistic or socialistic policy?)

- The respondent may not know anything about perestroika, but have a general opinion about it due to the media; therefore, an opinion is given as an answer.

- Another good case for "don't know" responses.

- If a question requires a numerical answer--make it open ended, this will avoid giving away the answer.

- Remember "don't know" is acceptable.

- Always end the survey with a "Thank You."

Attitude Questions

- The first consideration, when designing attitude questions, must be to clarify the attitude object (subject/topic/focus).

- Once the attitude object is clear, three aspects concerning it must be considered:

o cognitive - what they think or know about the object

- affective - whether they like/dislike, approve/disapprove, etc. the object

o action - what is the willingness for the respondent to do something in regard to the object.

- Once the respondent has chosen a direction in relation to the attitude object, strength questions must be built in to measure degrees of intensity associated with the direction.

- Questions measuring attitudes must take careful caution to avoid "double barreled" and "one and a half barreled" questions. These questions will surely compromise the validity of the questionnaire.

- Unipolar questions may be better suited for providing more specific results to attitude questions than Bipolar questions. Keep in mind, as mentioned above, that bipolar questions may reveal broader dimensions within attitudes.

- Always end the survey with a "Thank You."

Threatening Questions

- Knowledge questions can be threatening.

- Demographic questions can be threatening.

- Socially undesirable questions are threatening.

- Open questions are best used for threatening questions.

- Long questions, with socially/culturally familiar words, are best used for threatening and socially undesirable topics or behaviors.

- Remember, not to begin with threatening questions. Start with the least threatening, then increase the threat as necessary throughout the survey.

- The level of threat should be defined through the eyes of the respondent, not the researcher. 
- Informants are considered the most accurate means of obtaining threatening and socially undesirable data. Any respondent can be considered an informant by simply asking them to comment on the behavior of others.

- The perception of threatening, or undesirable topics may be reduced by embedding the topic into a list of either more or less threatening phrases, or topics. This can reduce the perceived importance to the threatening topic and reduce over or under reporting.

- When asking about socially undesirable behaviors, it is best to ask about past behavior first (e.g., Have you ever engaged in the specific behavior?), then move to the present.

- Socially desirable behavior is better asked in the form of "current" behavior.

- Intentionally loading questions that address socially undesirable behavior may actually reduce the perceived threat posed by that question, had it not been loaded. There are many techniques for intentional loading; two examples include:

- The everybody does it technique (e.g., Loaded - We all know it is common to see people exhibit xxx behaviors. Have you ever exhibited this xxx behavior in the past? Unloaded - Do you participate in xxx behavior?).

- The reason why approach (e.g., Loaded - Do you think soldiers who have come back from 3-day combat missions and are obviously exhausted, should be forgiven for forgetting to clear their weapon and negligently discharging it? Unloaded - Do you think a soldier should be forgiven for negligently discharging a firearm?).

- Have others comment on the threatening nature of questions during piloting.

- You may include questions at the end of the survey that rank the perceived threat of your questions and topics.

- Always end the survey with a "Thank You."

There are many more techniques, principles, and recommendations to include and

discuss. One thing that is important to understand is that asking questions and creating a questionnaire is probably much more of an "art" than objective "science." Therefore, there is probably an exception to everything mentioned in this paper. "Thank You"

\section{REFERENCES}

Bradburn, N.M., Sudman, S., \& Associates. (1979). Improving Interview Method and Questionnaire Design. San Francisco: Jossey-Bass.

Converse, J.M., \& Presser, S. (1986). Survey Questions: Handcrafting the Standardized Questionnaire. Beverly Hills, CA: Sage Publications.

Payne, S.L. (1951). The Art of Asking Questions. Princeton, NJ: Princeton University Press.

Sudman, S. \& Bradburn, N.M. (1983). Asking Questions: A Practical Guide to Questionnaire Design. San Francisco: Jossey-Bass Publishers. 
Appendix 3.3 - DCAC Data Management Plan 


\section{Data Management Plan}

Protocol:

Study:

Principal Investigator $(\mathrm{PI})$ :

DMP Version:

Date:
RV340

USASOC Health Behavior KAP Questionnaire

Major Michael J. Pagel,

WRAIR U.S. Army Public Health Command

0.1

280ct2011

Prepared by:

Greg Pfeiffer, Lead Data Management Specialist (DMS)

The United States Military HIV Research Program

(MHRP) Data Coordinating and Analysis Center (DCAC)

6720-A Rockledge Drive

Bethesda, MD 20817

Reviewed by:

Major Michael J. Pagel:

Principle Investigator

Date

\section{Overview}

This Data Management Plan (DMP) outlines policies and procedures related to data collection, entries, cleaning, transfer, processing and coding; as well as study database design and structure for RV340.

This DMP is prepared and distributed by DCAC in coordination with the PI. The DMP will be updated as necessary throughout the course of the study. Individuals may propose changes to the DMP at any time by submitting the change request to DCAC. DCAC staff will consult with the investigative team on all modifications impacting policies or procedures. (Editorial changes and clarifications may be made without consultation.) 


\section{Roles and Responsibilities}

Below describes the responsibilities of DCAC staff. Please see the protocol for roles and responsibilities of investigators and other study personnel.

\begin{tabular}{|c|c|}
\hline Staff & Responsibilit \\
\hline Rory Deshano & \\
Senior Program Manager & Department Manager \\
\hline $\begin{array}{c}\text { Mark Milazzo } \\
\text { Director of Technical Operations/ } \\
\text { MHRP Team Lead }\end{array}$ & $\begin{array}{c}\text { Data } \\
\text { Custodian } \\
\text { MHRP Team } \\
\text { Lead }\end{array}$ \\
\hline Jun Tian & Database Post- \\
& Processing \\
Programmer Analyst (PA) & Programmi \\
\hline Greg Pfeiffer & Study \\
Setup \\
Data Management & Database Creation \& \\
Specialist & Maintenance Data Processing \\
& $\&$ Management Data Entry \& \\
& Verification Documentation \\
DI
\end{tabular}

\section{Data Management Milestones}

Questionnaire finalization

Database design and testing

Data validation, programming and

testing Last data element (questionnaire) received Database audit or quality assurance review 
Database lock

Data and documentation archiving

\section{Data Collection Instruments}

A single 65-page questionnaire will be anonymously administered once to each participating Ranger (participant). Target administration will be to have a minimum of 124 completed questionnaires, and an oversampling maximum of 150 to anticipate potential drop-outs (participants administered the questionnaire, but not able complete it). Each participant consented to the study (with an anonymous Consent Form) will not have any identifiers or Personally Identifiable Information (PII) asked for or captured, or any Personal Identification Number (PIN) or Subject ID associated in any database. A list of 200 ID numbers will be generated by DCAC and pre-printed on each questionnaire. The ID number is only used to identify each questionnaire for data processing purposes, and cannot be linked to any participant.

\section{Collection schedule}

Recruitment of participants for this project has been conducted by the PI. If needed, the co- investigators may also recruit as requested by the PI. The PI will coordinate recruitment activities with the respective Battalion Commanders and medical staff. Dates and times may vary based on participant and unit availability.

\section{Data Flow}

There will be a single database developed in ClinPlus ${ }^{\circledR}$, which will be generated and maintained solely at DCAC. Questionnaires will be identified only by their ID number. All questionnaires will be sent to DCAC for processing. All data requested by the PI will be handled in a secure manner. The SPSS $®$ files requested will either be sent via MHRP Secure File Transfer System, or be personally delivered to the PI via flash drive by DCAC.

\section{Analysis}

The PI will receive the data in the requested format, SPSS $®$, and will be responsible for analysis.

\section{Database Specifications}

Data will be captured and reside in the $S A S \circledast$-based ClinPlus $®$ database software by DCAC, DZS as vendor. The database will live at DCAC headquarters located at $6720-A$ Rockledge Drive, Bethesda, MD. ClinPlus $®$ is a secure, password-protected, validated system that limits who has access, and the level of access to study data. The database will first be tested thoroughly and all data will be double-entered and verified. All DCAC standard Quality Control procedures will be performed.

Once cleaned, the data will be converted to SPSS $®$ files for the Pl's analysis. At the request of the PI, the variable names for all questions will be defaulted to the question's numeration on the questionnaire itself. The annotated value lists will be provided as well. 


\section{Database Testing}

Testing is conducted by completing "mock" questionnaires, then entering and verifying the questionnaires through double data entry. Any errors in the design or functionality of the application will be corrected and re-tested.

All $S A S \circledR$ import programs for the questionnaire data will be tested after the design and application testing is complete. Data exports will be compared to the raw data. Any errors in the program will be corrected, documented, and re-tested. This is completed according to DCAC SOP\#18.

\section{Participant Anonymity}

Participants will not be asked to identify themselves in any way on the questionnaire. No specific PII will be asked within the questionnaire. Each questionnaire will be identified only with the random four digit ID number preprinted on each page of the questionnaire. Participants will not be associated with any randomly assigned ID numbers in any database or hard copy document. No names or PII will be associated with any document or questionnaire. The PI or approved co- investigator will thoroughly instruct all participants that they are not to mark or place any PII on any of the pages.

If any PII is present, it will be flagged immediately and the Lead DMS will be notified. The Lead DMS will alert the PI immediately so he can readdress the issue to further participants. The Lead DMS will determine if the mark(s) is definitive or potential PII. If any potential PII s present, it will be marked over in black permanent marker and rendered unreadable by the Lead DMS. If any definitive PII is present, the Questionnaire is to be destroyed.

\section{Data Collection and Management}

The questionnaires will be printed on spiral-bound booklets by HJF Printing and Graphics department, located at MHRP headquarters. The Lead DMS will receive and transmit the questionnaires to the PI via FedEx, including a detailed Transmittal form to ensure the integrity of the contents. Data will be collected solely from the questionnaires that each participant completes.

The questionnaire is organized into 5 sections: Demographics (no specific PII involved), Malaria Chemoprophylaxis, Condom Use, Weapon Safety, and finishing with three survey administrative questions. The Malaria Chemoprophylaxis, Condom Use, and Weapon Safety sections are each divided into subsections: General Knowledge, General Attitudes, Descriptive Norms, Injunctive Norms, Perceived Susceptibility, Behavior Outcome Related Practices, Interpersonal Communication, Behavior Related Education, Behavior Related Training, Behavior Related Media, and Command Support.

Each potential participant will be given a thorough study briefing by either the PI or an IRB approved co-investigator. This briefing will include a summary of the study and its objectives. The briefing will also include anticipated risks, benefits, and alternative to participation. Each participant must be a volunteer and complete the IRB approved anonymous consent form prior to beginning the survey questionnaire. Each participant will be given a large manila envelope, a blank questionnaire booklet, and a blue pen. The participants will place the 
questionnaire into the manila envelope and seal it prior to returning it to the PI or coinvestigator. A randomly generated identification number is printed on every page within the questionnaire booklet, but again cannot be used to identify the participant. This randomly assigned number will also be placed on the outside of the manila envelope to maintain transmittal form tracking. The PI or co- investigator will maintain all anonymous consent forms; DCAC does not require their submission. Participants will be instructed to complete each question in the questionnaire. The PI or an approved co-investigator will remain available to answer any participant questions and address any issues.

The PI or an approved co-investigator will collect the enveloped questionnaires, complete the questionnaire Transmittal Form, package them for shipment, and send the completed and enveloped questionnaires via FedEx to DCAC. Each shipment will contain a copy of a completed Transmittal form.

\section{Data Entry and Processing}

The Lead DMS will receive and process the questionnaires. Questionnaires will be tracked in a Document Tracking spreadsheet that includes the questionnaire ID number, date sent out from DCAC, date received by DCAC for processing, and date verified in ClinPlus $₫$. DCAC staff will contact the PI if any discrepancies are found between the Transmittal form and the shipment received. Questionnaires that the PI has reported lost will be documented as "Not Returned" in a separate log.

DEls have been prepared and made available in PDF format for data entry staff. Also, a SelfEvident Corrections (SECs) document has been prepared and distributed to all data entry staff, instructing their appropriate application concerning 3 questions within the questionnaire. Data Entry Logs will be prepared by the Lead DMS, and placed atop the questionnaires to be distributed to trained data entry staff. After first entry into ClinPlus $₫$, the Lead DMS or designee will conduct the second entry and verification, and ensure all SECs have been correctly captured. The Lead DMS will then update the Document Tracking Log and SEC Log. The Data Entry Logs will be securely filed along with the questionnaires in accordance with DCAC SOP\#14

Determining File Storage.

\section{Data Validation}

Since the questionnaires are administered anonymously and only a single study visit is performed, no data queries or corrections will be made. Self Evident Corrections will be allowed only for 3 specific questions, as detailed in the RV340 SEC document. All questionnaires submitted to DCAC will be accepted for data entry. Handling of nonresponses or inconsistent data will be the responsibility of the PI (who is performing the data analysis).

\section{Quality Control}

Once data Verification in ClinPlus $®$ is complete and all applicable SECs have been made, DCAC will perform a 10\% Quality Control check of verified questionnaires. This involves a visual comparison of the questionnaire data to the study database. QC will be performed according to DCAC SOP\#28: Quality Control. 
Error rates by page, by observation, and overall will be generated. If the observational error rate is above $0.1 \%$ then another $10 \%$ QC will be performed. Any additional queries or corrections prompted by the QC will be resolved prior to database lock.

\section{Security Measures}

All data will be kept on a password-protected server, and files stored within the locked file cabinets of study personnel and will only be available to authorized personnel. DCAC data security measures are outlined in DCAC SOP\#35. Researchers will be required to provide proof of completion of appropriate CITI training prior to beginning any research activities.

No PII of any kind (including names, national identification numbers, dates of birth, etc.) will be transmitted to DCAC, as PII will not be associated with any document or asked for on the questionnaires. The PI or approved co-investigator will thoroughly instruct all participants that they are not to mark or place any PII on any of the pages. If any PII is present, it will be flagged immediately and the Lead DMS will be notified. The Lead DMS will alert the PI immediately so he can readdress the issue to further participants. The Lead DMS will determine if the mark(s) is definitive or potential PII. If any potential PII s present, it should be marked over in black permanent marker and rendered unreadable by the Lead Data Manager. If any definitive PII is present, the Questionnaire is to be destroyed.

All data requested by the PI will be handled in a secure manner. SPSS $₫$ files requested by the PI will be sent via the MHRP Secure File Transfer System, or be personally delivered from the DCAC to the PI via flash drive.

\section{Database Lock/Freeze}

After study completion, the database will be locked and archived according to DCAC SOP\#26: Database Lock.

\section{Questionnaire Archiving}

After final study database lock, the DMS will initiate the process for archiving the questionnaires according to DCAC SOP\#32, Data Archiving.

\section{DMP Change Control Log}

\begin{tabular}{|l|l|l|l|}
\hline Date & Version & Author & Revision \\
\hline $\begin{array}{l}\text { Date of } \\
\text { revision }\end{array}$ & New version & $\begin{array}{l}\text { Name of person } \\
\text { making revision }\end{array}$ & $\begin{array}{l}\text { Describe revision made, where } \\
\text { located and why }\end{array}$ \\
\hline
\end{tabular}

\section{References}

1. DCAC SOP\#14-Determining File Storage

2. DCAC SOP\#18-Programming

3. DCAC SOP\#26-Database Lock 
4. DCAC SOP\#28-Quality Control

5. DCAC SOP\#32-Data Archiving

6. DCAC SOP\#35-Data Security

\section{Appendices}
1. Transmittal Form
2. Data Entry Instructions
3. Document Tracking Log
4. Data Entry Log
5. Not Returned Document Tracking Log
6. Quality Control Log
7. Self-Evident Corrections Instructions
8. Self-Evident Corrections Log
9. Study ID numbers
10. Annotated Value Lists 
Appendix 3.4 - Institutional Review Boards 


\section{Institutional Review Boards}

To insure the full protection of human subjects, this research was conducted in full compliance with all required Institutional Review Boards (IRB): there were two IRBs required for this research.

The Florida International University (FIU) was the primary IRB for this study. Due to funding support, the WRAIR was also required to be a separate and independent military IRB approving authority.

The FIU IRB did not initially require the additional investigators (Dr Scott and Major Gilpatrick) as did the WRAIR IRB. These IRBs were later reconciled in a formal amendment to the research protocol. Curriculum vitae(s) and proof of required Human Subjects training were supplied to the each of the IRB offices.

On June 28, 2011 the FIU IRB determined this protocol to meet exemption criteria and approval from the Division of Human Subjects Protection (DHSP) was received on July 20, 2011 as FIU IRB Exemption \#062811-01. An additional statement accepting non-substantial changes to the study questionnaire (version 1.1) was received from the FIU IRB on July 27,2011 which authorized small grammatical changes to the survey instrument questionnaire; which was then noted as version 1.2. On October 31, 2011 the FIU IRB, via expedited review procedures, approved the substantive changes to this protocol which included:

1. Reconciling the inclusion of additional Investigators (Dr Scott and Major Gilpatrick) to the FIU IRB which were not initially of them but were required by the WRAIR IRB.

2. Increasing the sample size from 124 to 150 participants.

3. Updating minor grammatical changes within the protocol documents.

4. Updating the Anonymous Informed Consent from Version 1.0 to Version 1.1 which included changing the stated sample size increase from 124 to 150 . 
5. Updating changes to the Study Protocol from version 1.0 to 1.1 by noting the changes in the sample size and power analysis information within Subject Recruitment and Methods sections.

The WRAIR IRB also found this protocol to be exempt on June 24, 2011; accepting the study as WRAIR \#1851, RV 340. They too formally noted the questionnaire changes on July 27 , 2011. Additionally, WRAIR formally approved the amendment to this exempt protocol, echoing the changes noted above, on November 4th, 2011.

Close out reports are due to both IRBs upon completion of the study. 
Appendix 3.5 - Power Analysis Protocols 


\section{Post Hoc power analysis for 150 participants}

[1] -- Thursday, June 14, 2012 -- 12:28:58

z tests - Logistic regression

Options: Large sample z-Test, Signorini (1991)

Analysis: Post hoc: Compute achieved power

Input:

Tail(s)

Odds ratio

$\operatorname{Pr}(\mathrm{Y}=1 \mid \mathrm{X}=1) \mathrm{H} 0$

$\alpha$ err prob

Total sample size

$\mathrm{R}^{2}$ other $\mathrm{X}$

$X$ distribution

$X$ parm $\mu$

$X$ parm $\sigma$

Output: $\quad$ Critical $z$

Power (1- $\beta$ err prob)
= Two

$=2.3333333$

$=0.3$

$=0.05$

$=150$

$=0$

$=$ Normal

$=0$

$=1$

$=1.9599640$

$=0.9974089=99.7 \%$

\section{Second power analysis for 150 participants}

[1] -- Sunday, October 16, 2011 -- 10:37:21

Exact - Correlation: Bivariate normal model

Options: exact distribution

Analysis: A priori: Compute required sample size

Input:

$$
\begin{array}{lll}
\text { Tail }(\mathrm{s}) & = & \text { Two } \\
\text { Correlation } \rho \mathrm{H} 1 & & 0.3 \\
\alpha \text { err prob } & = & 0.05 \\
\text { Power }(1-\beta \text { err prob) } & = & 0.964 \\
\text { Correlation } \rho \mathrm{H} 0 & = & 0 \\
\text { Lower critical } r & = & -0.1603348 \\
\text { Upper critical } r & =0.1603348 \\
\text { Total sample size } & =\mathbf{1 5 0} \\
\text { Actual power } & =0.9643997=\mathbf{9 6 . 4 \%}
\end{array}
$$

Output: Lower critical $r$

[1] -- Sunday, October 16, 2011 -- 11:12:53

z tests - Logistic regression

Options: Enumeration method, Wald-test

Analysis: A priori: Compute required sample size Input:

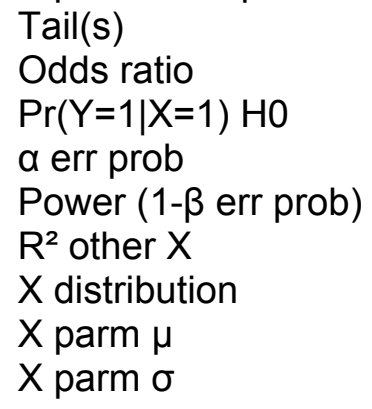

= Two

$=2.3333333$

$=0.3$

$=0.05$

$=0.978$

$=0$

$=$ Normal

$=0$

$=1$ 
Output: Noncentrality parameter $\lambda=15.8454037$

Critical $X^{2} \quad=3.8414588$

Df $\quad=1$

Total sample size $\quad=150$

Actual power $\quad=0.9783428=\mathbf{9 7 . 8 \%}$

Initial power analysis for 124 participants

Derived from the highest requirement of $112+10 \%$ over estimate of $11.2=123.2$, rounded up to $\underline{124}$

[2] -- Wednesday, February 02, 2011 -- 20:00:02

Exact - Correlation: Bivariate normal model

Options: exact distribution

Analysis: A priori: Compute required sample size

Input: Tail(s) = Two

Correlation $\rho \mathrm{H} 1 \quad=0.3$

$\alpha$ err prob $\quad=0.05$

Power (1- $\beta$ err prob) $\quad=0.9$

Correlation $\rho \mathrm{HO} \quad=0$

Output: Lower critical $r \quad=-0.1856685$

Upper critical $r \quad=0.1856685$

Total sample size $\quad=112$

Actual power $\quad=0.9008078$

[8] -- Wednesday, February 02, 2011 -- 20:03:49

z tests - Logistic regression

Options: Enumeration method, Wald-test

Analysis: A priori: Compute required sample size

Input: Tail(s)

Odds ratio

$\operatorname{Pr}(\mathrm{Y}=1 \mid \mathrm{X}=1) \mathrm{HO}$

= Two

$\alpha$ err prob

$=2.3333333$

Power (1- $\beta$ err prob)

$=0.3$

$R^{2}$ other $X$

$=0.05$

$\mathrm{X}$ distribution

$=0.90$

$X$ parm $\mu$

$=0$

$=$ Normal

$X$ parm $\sigma$

$=0$

$=1$

Output: Noncentrality parameter $\lambda=10.5280093$

Critical $X^{2} \quad=3.8414588$

Df

$=1$

Total sample size

$=100$

Actual power

$=0.9005560$ 
Appendix 3.6 - Anonymous Consent Form 


\section{Knowledge, Attitudes, And Practices Affecting Health Behaviors In A U.S. Army Special}

Operations Command Population of Rangers - Anonymous Informed Consent Briefing

Informed consent to participate in the survey questionnaire will be given anonymously by each participant by reading this document and circling the appropriate responses. You will not be linked to this form in any way.

Prior to reading this section of the informed consent, a full explanation of the purpose, and objectives of the study will be explained to you and made available for you to read. If you have any questions, please ask at any time. See the Study Summary on the following page.

You must be 18 years or older to participate.

Following are the perceived risks, benefits, and alternatives to participation:

Risks - The risks are perceived as minimal and include:

- The possibility of misunderstanding, or misinterpretation of a question or statement in the questionnaire.

- There are no other foreseeable risks.

Benefits - The benefits of your participation may include:

- You may gain an better understanding of issues that may affect health behaviors.

- You may help improve overall health outcomes in military populations.

- Your participation may help improve adherence to health behaviors.

- Your participation may help decrease the incidence of malaria.

- Your participation may help improve patient/provider relationships.

Alternatives to participation - Alternatives to participation are simply "not" to participate. You are not obligated in any way to participate, and you may withdraw at anytime.

Strict confidentiality will be maintained with all information given in the questionnaire, it will not be revealed to anyone outside the research team, and you will remain absolutely anonymous with no link between you and your responses.

Participation is voluntary. If you do not wish to participate, there will be no penalty or loss of any benefits you may be currently or otherwise entitled to. You may discontinue participation at any time as well, without penalty or loss of benefits to which you are otherwise entitled to.

You may contact Major Michael Pagel at any time during this survey today, or later by telephone at 786-255-3132, or email at michael.pagel1@us.army.mill for an explanation or answers to any questions concerning this research or your rights as a participant; particularly if you feel you have been offended, injured, or harmed in any way from this study.

Do you fully understand the purpose, duration, and procedures of the study?

Yes / No

Do you fully understand the risks, benefits, and alternatives to participation?

Yes / No

Do you understand your participation is completely voluntary?

Do you understand you may withdraw at any time?

Do you consent to participate by completing this questionnaire?

Are you 18 years old or older?

Yes / No

Yes / No

Yes / No

Yes / No 


\section{Study Summary}

Background: Malaria is a deadly and preventable threat present throughout much of the US Army Special Operations Command (USASOC) area of responsibility. Malaria chemoprophylaxis medications can help prevent malaria infections. When properly prescribed and adhered to, these medications are very effective, but require $100 \%$ adherence to ensure protection. Studies consistently find very low levels of adherence to these medications; usually from around $10 \%$ to highs around $50 \%$. A significant gap exists in the knowledge concerning adherence to these medications in our military, particularly within the USASOC populations. No available literature exists that thoroughly examines the Knowledge, Attitudes, and Practices (KAPs) affecting this behavior in this population. This is what you are helping to improve.

Method: A cross sectional questionnaire will be administered that simultaneously examines adherence to three health behaviors: 1-highly adhered to behavior (weapon safety, which is greater than $99 \%$ adhered to), 1-medium adhered to behavior (condom use, which is $43 \%$ adhered to in the Army), and 1-low adhered to behavior (malaria chemoprophylaxis medications, which are adhered to by $10-50 \%$ of military personnel). Each behavior has its own section. The questionnaire is anonymous and will be administered here at your place of duty. You must be a volunteer and over 18 years of age to participate. We want to have at least 124 participants from the Ranger units, but may sample as many as 150 . There are no experimental procedures in this study.

Duration: It takes approximately $\mathbf{9 0}$ minutes to complete the entire questionnaire. That is all the time you will be asked to give. You may take a restroom break at any time.

Transparency: All study practices and procedures have been provided to the USASOC leaders, as well as the medical and public health offices. Nevertheless, all the information you give will remain confidential and you will remain anonymous. All funding, labor, and logistical support has been provided by Major Michael J. Pagel and the Walter Reed Army Institute of Research (WRAIR).

Findings: We are looking for the KAPs and the corresponding levels of reinforcement that may affect adherence to Weapon Safety, Condom use, and Malaria Chemoprophylaxis medications. We will also collect the current levels of adherence you report for each behavior. We intend to use what is working for one behavior to improve what is not working in another.

Purpose 1: Identify the primary issues that affect adherence to the three behaviors.

Purpose 2: Identify the impact of reinforcement on each behavior.

Purpose 3: Understand how these factors can be used to improve future efforts to improve adherence to malaria chemoprophylaxis medications.

The Study Findings will: (a) add to the current base of knowledge; (b) guide future interventions at both the medical and combat operations level; and (c) improve the future health, readiness, and effectiveness of USASOC personnel, particularly those who venture or deploy to malaria endemic areas. 
Appendix 4.1 - Significant Pearson Correlations for Condom Use and Weapon Safety 
Interpretations of Catalog of Research Findings: Condom Use, Bivariate Correlations.

Significant Bivariate Correlation relationships exist between the following variables and the behavior outcome C34A (Outside a long-term and strictly monogamous relationship: Did you yourself use a condom during every act of sexual intercourse?):

Variable

Pearson Correlation

C29E.wDs (Gradable Knowledge) Can STIs cause death? $-.267^{* *}$

C29G.wDs (Gradable Knowledge) How do condoms help prevent? $.240^{*}$

C29H.wDs (Gradable Knowledge) Which condom should not be used? $.227^{*}$

C29M. (Gen Know) In past 24-36 months, had sex? $.239^{*}$

C30G.wDs Think of punishments if not using $.300^{* *}$

C30H.wDs Punishments influence you? $.462^{* *}$

C32D.wDs Friends think it's OK if I forget? $-.227^{*}$

C32H.wDs Friends think it's OK if I recommend $.205^{*}$

C34B.wDs How often do you use a condom? $.404^{* *}$

C34C.wDs Use a condom during last act of sex. $.347^{* \star}$

C34D.wDs Fear the repercussions if I catch STI. $.367^{* *}$

C38B.wDs STI danger messages were informative? $-.544^{*}$

C38E.wDs Paid attention to STI messages? $-.386^{*}$

*. Correlation is significant at the 0.05 level (2-tailed).

${ }^{* *}$. Correlation is significant at the 0.01 level (2-tailed).

Note: Only Medium and Strong correlations are reported; nevertheless, correlations greater than \pm 0.200 are reported when no Medium or Strong correlations were identified. Strong correlations are identified with an underline. 
Significant Bivariate Correlation relationships exist between the following variables and the behavior outcome C34I (Do you plan to use a condom the next time you have sexual intercourse outside a strictly monogamous relationship?):

\section{$\underline{\text { Variable }}$}

C30F1.wDs How much of a hassle?

C33F.wDs Influence you to use a condom?

C34B.wDs How often do you use a condom?

C34C.wDs Use a condom during last act of sex.

C34H.wDs I plan condoms into my missions?

C34B.wDs (Ever/Never) How often do you use a condom?

C34H.wDs (Ever/Never) I plan condoms into my missions?

C35A.wDs Had discussions with friends to use condoms.

*. Correlation is significant at the 0.05 level (2-tailed).

**. Correlation is significant at the 0.01 level (2-tailed).

Note: Only Medium and Strong correlations are reported; nevertheless, correlations greater than \pm 0.200 are reported when no Medium or Strong correlations were identified. Strong correlations are identified with an underline.

\section{Pearson Correlation}

$-.391^{* *}$

$.313^{* *}$

$.510^{* *}$

$.342^{* *}$

$.338^{*}$

$.515^{\star *}$

$.445^{* *}$

$.225^{*}$

\author{
rong
}


Interpretations of Catalog of Research Findings: Weapon Safety, Bivariate Correlations.

Significant Bivariate Correlation relationships exist between the following variables and the behavior outcome W45A (Do you yourself follow the recommended weapon safety guidance at all times?):

$\underline{\text { Variable }}$

W41B.wDs: Well informed why to follow guidance?

W41E.wDs: Following safety at all times is a hassle?

W42H.wDs: Others feel guidance is always appropriate?

W45B.wDs How often do you follow guidance?

W49A.wDs Messages posted-around unit, dangers of weapons

W49C.wDs Messages posted-around unit, follow safety guidance

W50F1.wDs How often recall this required

W50F1.wDs (Ever / Never) How often recall this required

*. Correlation is significant at the 0.05 level (2-tailed).

${ }^{* *}$. Correlation is significant at the 0.01 level (2-tailed).

Note: Only Medium and Strong correlations are reported; nevertheless, correlations greater than \pm 0.200 are reported when no Medium or Strong correlations were identified. Strong correlations are identified with an underline.

No Significant Bivariate Correlations exist between any independent variables and the behavior outcome $\mathbf{W} \mathbf{4 5 H}$ (Do you plan to follow the recommended weapon safety guidance the next time you operate a weapon?)

\section{Pearson Correlation}

$.341^{* *}$

$-.398^{* *}$

$.243^{* *}$

$.490^{* *}$

$.266^{\star *}$

$.260^{*}$

$.211^{*}$

$.229^{* *}$ 


\section{Appendix 4.2 - Correct Survey Questionnaire Knowledge Responses}

Note: Gradable knowledge questions are highlighted and the correct responses are noted in red font. 
ID\#|_ا_Lا_I

General Knowledge of Malaria and Chemoprophylaxis Medications

M18. The next set of questions considers your knowledge regarding malaria and malaria chemoprophylaxis medications.

For each question, please provide the "single" best answer. If you don't know the answer to the question, it is alright to say that you don't know. You may also refuse to answer the question. Do not hesitate to ask for help if you do not understand.

M18A. Have you ever heard of malaria? Not Gradable, General Knowledge
O. No
Don'tknow
○ 1. Yes
Refuse to Answer

M18B. Can a person get malaria from a mosquito bite?
O. No
1. Yes
Don't Know
Refuse to Answer

M18C. Can a person get malaria from blood transfusions?
O. No
Don't Know
(1. Yes
O Refuse to Answer

M18D. Can malaria be transmitted from person to person by close contact or cough?
O D. No
O Don't Know
O 1. Yes
O Refuse to Answer

M18E. Can malaria cause death?
O D. No
O Don't Know
O 1. Yes
O Refuse to Answer

M18F. Have you ever been prescribed malaria chemoprophylaxis medications? Not Gradable, General Knowledge
O D. No
O 1. Yes
Don't Know
Refuse to Answer 
M18G. What do malaria chemoprophylaxis medications do? (Please choose only one best answer.)
O 1. Prevent mosquito bites
O 2. Prevent malaria from developing in your body
O Don't Know
O 3. Prevent both HIV and Malaria
O 4. Make you permanently immune to Malaria

$\mathrm{M} 18 \mathrm{H}$. Which medication is "not" a malaria chemoprophylaxis medication?
O 1. Mefloquin
2. Doxycycline
Q Don't Know
O. Penicillin
Ǒ 4. Chloroquine
Refuse to Answer

M181. Can malaria chemoprophylaxis medications be stopped immediately after leaving the area where malaria is present?
$\begin{array}{ll}\text { O } & \text { 1. No } \\ \text { O. Yes }\end{array}$
O Don't Know

M18J. Can missing a single dose of malaria chemoprophylaxis medications put you at greater risk for contracting malaria?
O 0 . No
O Don't Know
O 1. Yes
O Refuse to Answer

M18K. Which form of malaria prevention is the most effective?
O 1. There is no effective form of Malaria prevention
O 2. Taking Malaria Chemoprophylaxis Medications
O Don't Know
O 3. Avoidance with repellents, nets, and protective clothing
O 4. Combination of Medications and Avoidance

M18L. Can the military punish you for not taking your malaria chemoprophylaxis medications as recommended?
$\begin{array}{ll}\text { O } & \text { No No } \\ \text { 1. Yes }\end{array}$
O Don't Know 
M18L2. If there are any punishments or repercussions: Can you please choose which of the following punishments or repercussions you think would be the most likely to occur? Please choose only one. Not Gradable, General Knowledge

ญ̊

1. Article 15 , with reduction in rank

2. Article 15 , with loss of some pay

3. Court martial proceedings

4. Some type of limited punishment, such as extra duty

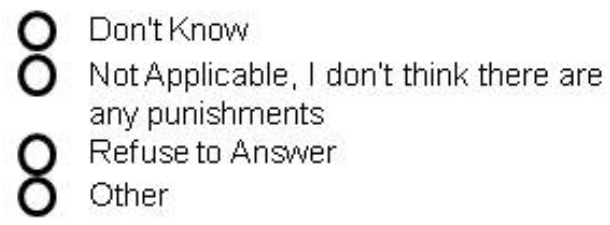

M 18M. In the past 36 months: Have you yourself been deployed to an area where malaria was present?

Not Gradable, General Knowledge

O O. No

O 1. Yes

Don't Know

Refuse to Answer

M 18M 1. In the past 36 months: Have you yourself been deployed to Afghanistan, Pakistan, India, any country in South or Central America, Haiti or the Dominican Republic, any country in Africa, or any country in Southeast Asia? Not Gradable, General Knowledge
O 0 . No
O Don't Know
O 1. Yes
O Refuse to Answer

M18N. How long were you actually in the area where there was a risk of contracting malaria? Not

Gradable, General Knowledge

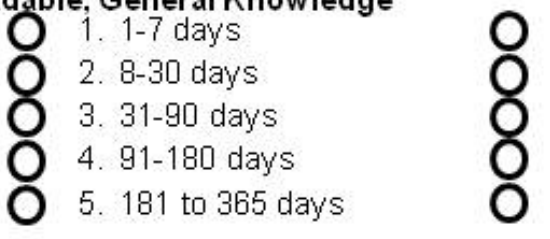

6. Between 1 to 2 years

7. More than 2 years

Don't Know

NotApplicable, I was never deployed to an area with Malaria

Refuse to Answer

M180. Have you yourself known of any people, including civilians, being infected with malaria in places where you have been deployed? Not Gradable, General Knowledge
O 0. No
O Don't Know
O 1. Yes
O Refuse to Answer

M18P. Can you get sick from malaria after you have returned to your home unit back in the US?
$\begin{array}{ll}\text { O } & \text { 0. No } \\ \text { O } & \text { 1. Yes }\end{array}$
O Don't Know 
Note: The term Sexually Transmitted Disease, also referred to as an STD, is now more commonly referred to as a Sexually Transmitted Infection. Throughout this section of the questionnaire, Sexually Transmitted Infection will often be abbreviated as STI.

Additionally: Many of these questions may not relate well to practices in long-term and strictly monogamous relationships, where condom use may not be regularly practiced.

If you happen to be in one of these relationships and do not regularly use a condom, please feel free to choose the Not Applicable option when provided.

Otherwise, choose the answer that best describes how condom use relates to you.

C29. The next set of questions considers your knowledge regarding condoms.

For each question, please provide the "single" best answer.

If you don't know the answer to the question, it is alright to say that you don't know.

You may also refuse to answer the question.

Do not hesitate to ask for help if you do not understand.

C29A. Have you ever used a condom? Not Gradable, General Knowledge
O 0. No
Q Don't Know
O 1. Yes
Refuse to Answer

C29B. Can a person get Sexually Transmitted Infections (STIs) from improper use of condoms?
O D. No
1. Yes
O Don't Know
Refuse to Answer

C29C. Can a person get some of the same STIs from blood transfusions?
O 0 . No
Q Don't Know
O 1. Yes
Refuse to Answer

C29D. Which is "not" an example of an STI? (Please choose only one best answer)
(1. Gonorrhea
O 2. HIV (Human Immunodeficiency Virus)
- 4. Malaria
3. Chlamydia
O Don'tknow
(O)fuse to Answer

C29E. Can STIs cause death?
O 0 . No
Q Don't Know
O 1. Yes
Refuse to Answer

C29F. Have you ever been given a condom to prevent STIs? Not Gradable, General Knowledge
O 0. No
O 1. Yes

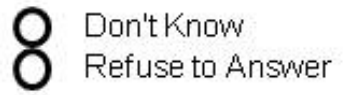


C29G. How do condoms help to prevent Sexually Transmitted Infections (STIs)? Not Graldäle,betherat

\section{Knowledge}

O 1 . By removing infectious organisms

O 4. By creating immunity to STIs

2. By preventing organisms from contacting your tissue

3. By curing infections your partner

Refuse to Answer my have

$\mathrm{C} 29 \mathrm{H}$. Which type of condom should "not" be used for protection against STIs?

O 1. Colored, Latex, properly fitted

O 2. Ribbed, Latex, properly fitted

3. Edible, non-Latex, properly fitted
O 4. Textured, Latex, properly fitted

O Don'tknow

Refuse to Answer

$\mathrm{C} 291$. Are there different ways to prevent STIS, other than using condoms?
O 0 . No
O Don't Know
O 1. Yes
O Refuse to Answer

C29.J. Outside a strictly monogamous relationship: Is it recommended to use a condom only every once in a while?
O 0 . No
O Don't Know
O 1. Yes
O Refuse to Answer

C29K. Which is the most effective means of preventing Sexually Transmitted Infections (STIs)?

O 1 . There is no effective means of preventing STIS

O 2. Occasional condom use

O 3. Not having any oral, anal, or vaginal intercourse
O 4. Having unprotected intercourse with only 1 partner

O Don't Know

O Refuse to Answer

C29L. Can the military punish you for not using a condom during an act of intercourse?
$\begin{array}{ll}\text { O } & \text { 0. No } \\ \text { O } & \text { 1. Yes }\end{array}$

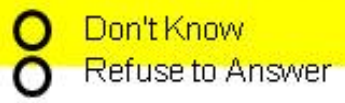

$\mathrm{C} 29 \mathrm{~L} 2$. If you believe there are any punishments, or repercussions: Can you please choose which of the following punishments or repercussions would be the most likely to occur?

Please choose only one answer. Not Gradable, General Knowledge

8

1. Article 15 , with reduction in rank

2. Article 15 , with loss of some pay

3. Court martial proceedings

4. Some type of limited punishment, such as extra duty
O Other

O Don't Know

O Refuse to Answer

O NotApplicable, I don't think there are any punishments 
C29M. In the past 24 to 36 months: Have you yourself had any type of sexual intercourse? Not Gradable,

General Knowledge
$人 \begin{aligned} & \text { 0. No } \\ & \text { 1. Yes }\end{aligned}$
Don't Know
Refuse to Answer

$\mathrm{C} 29 \mathrm{~N}$. Does sexual intercourse include oral, anal, and vaginal intercourse?
O. No
Don't Know
1. Yes
Refuse to Answer

C29N1. Which is "not" a form of sexual intercourse? (Please select only one answer)
1. Anal
4. Self Masturbation
2. Vaginal
Don't Know
O̧. Oral
O Refuse to Answer

C290. How many times have you had sexual intercourse in the past 24-36 months, with or without a condom? Not Gradable, General Knowledge
O 1. 1-5 times
6. 61 to 100 times
2. 6-10 times
O 7. More than 100 times
3. 11 to 20 times
4. 21 to 40 times
Don't Know
( 5. 41 to 60 times
Refuse to Answer
NotApplicable- I have not had any sexual intercourse in this period

C29P. Have you yourself known of people being infected with "any" Sexually Transmitted Infection (STI)? Not Gradable, General Knowledge
O D. No
O Don'tKnow
1. Yes
Refuse to Answer

C29Q. Can you become ill, or show signs of infection from an STI long after you have had any type of sexual intercourse?
O D. No
O Don't Know
1. Yes
C Refuse to Answer 


\section{General Knowledge of Weapon Safety}

Note: Weapon Safety can be a very relative term: it can have varying degrees of meaning to different groups. An extreme example of this may be that a safe weapon to a normal suburban housewife is one that is unloaded, also has a trigger lock, and is also locked up in a gun safe.

To Special Operations members, weapon safety may have a different meaning. For example, you often carry loaded automatic weapons that may be at the ready position. Therefore, your meaning of "safe" can be different than that of the suburban housewife's. Safe, to you, may mean that the risk of someone getting shot accidentally is minimized to the greatest extent possible, or that no weapon is discharged unintentionally.

Your concept of what a "normal" level of safety may be, as a Special Operations member, is what we would like you to refer to when answering this section of the questionnaire. Also, please do not consider extreme circumstances or uncommon military scenarios where safety may have been compromised, but no one was injured.

Additionally: These questions will refer to personal and crew-served weapons only. The questions in this section are not intended to be answered in relation to Artillery, Close Air Support, or other means of indirect supporting weapons.

W40. The next set of questions considers your knowledge regarding weapon safety. For each question, please provide the "single" best answer. If you don't know the answer to the question, it is alright to say that you don't know. You may also refuse to answer the question. Do not hesitate to ask for help if you do not understand.

M40A. Are you familiar with the operation of the following weapons: M 16 series rifles, M4 or M4A1 Carbine, M9 pistol, M240B Machine gun, M2 .50 Caliber Machine Gun, M249 Machine Gun, and the M 203 Grenade Launcher? Not Gradable, General Knowledge
O D. No
O Don'tknow
O 1. Yes
O Refuse to Answer

W40A1. If you are "not" familiar with the operation of any of these weapons:

Please check those you are "not" familiar with.

(Please answer all that apply, or Not Applicable if you are familiar with all.) Not Gradable, General Knowledge

Not Applicable, I am familiar with the operation of all these weapons
a. M16 series rifle
O e. M2.50 Caliber Machine Gun
b. M4 or M4a1 Carbine
O f. M249 Machine Gun
c. M9Pistol
g. M203 Grenade Launcher

d. M240B Machine Gun

O Don'tknow $\quad O$ Refuse to Answer

$\mathrm{R} \vee 340$

Questionnaire
8
Version 1.2

12. Mug $2011^{2}$ 
W40B. Can you be severely injured from the unsafe use of your weapon?
O 0. No
O Don't Know
1. Yes
Refuse to Answer

M40C. Can you be severely injured from another individual's unsafe use of their weapon?
O. No
1. Yes
O Don't Know
Refuse to Answer

M40D. Which is "not" a good example of weapon safety?
1. Clear all weapons at required clearing points

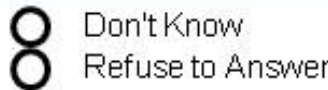
2. Provide and verify the correct ammo for all weapons
3. Ensure weapons are on safe when not being fired
4. Consider unloaded weapons as safe

W40E. Can the improper use of "any" weapon be deadly?
O 0. No
Don't Know
1. Yes
O Refuse to Answer

M40F. Which is "not" a correct performance step in zeroing the M4 or M4A1 Carbine?

1. Select the long-range aperture on the rear sight

O Don't Know

O 2. Center the rear sight aperture

O Refuse to Answer

O 3. Rotate the elevation knob counterclockwise to the 300 meter mark

- 4. Rotate the elevation knob 2 clicks counterclockwise below the 300 meter mark

M40G. Which is "not" a correct performance step in unloading the M4 or M4A1 Carbine?

1. Point the muzzle in a safe direction

2. Attempt to place the selector lever on SAFE

Don'tknow

3. Observe the magazine

4. Lock the bolt open and return the charging handle foward

W4OH. Which is "not" a correct performance step in the function check of an M240B M achine Gun?

1. Place safety lever to "F" position

2. Lock the bold to the rear by pulling cocking handle

O Don't Know

O 3. Ensure the cocking handle remains locked rearward

4. Place safety lever to "S" position

\begin{tabular}{lrr}
\hline RV340 & 9 & Version 1.2 \\
Questionnaire & 9 & 12 A $_{\text {ug } 2011}$
\end{tabular}


W40l. During Urban Operations:

Is it safe to practice pointing your weapon slightly downward in the direction of travel?
8
0. No
1. Yes
O Don't Know
O Refuse to Answer

W40.J. During Urban Operations: Is it safe to shoot while moving faster than you can engage your targets?
O 0 . No
Oे 1. Yes
O Don't Know
O Refuse to Answer

W4OK. During Urban Operations: Which movement technique is considered the "safest" ready position when moving in buildings or rooms? Not Gradable, General Knowledge
O 1. The standing unsupported
O 2. The low ready
O. The prone supported
4. The high ready
O D. No
O Don't Know
O 1. Yes

W40L. Can the military punish you for not following weapon safety guidance?

W4OL2. If you believe there are any such punishments: Can you please choose which of the following punishments or repercussions would be the most likely to occur? Please choose only one. Not

Gradable, General Knowledge 15 , wition in rank

O 2. Article 15, with loss of some pay

Q Don't Know

O Refuse to Answer

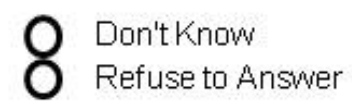

3. Court marital proceedings

4. Some type of limited punishment, such as extra duty

W40M. In the past 24 to 36 months, have you yourself had to practice weapon safety techniques during combat, live fire training, or other high risk military deployments? Not Gradable, General

Knowlèdge
O․ No
O Don't Know

W40N. If so, How long was your last high risk deployment, where you operated a weapon? Not Gradable,

General Knowledge
O 1. 1-7 days
Q 2. 8-30 days
3. $31-90$ days
O 6. Between 1 to 2 years
O 4. 91-180 days
Q 7. More than 2 years
Don't Know
5. 181 to 365 days
O NotApplicable, I was not deployed in this period
Refuse to Answer

W400. Have you known of people who were injured due to the unsafe operation of a weapon? Not

Gradable, General Knowledge
O 0 . No
O 1. Yes
8
Don't Know
Refuse to Answer 
ID\# |_ا_I_L_

General Knowledge of Malaria and Chemoprophylaxis Medications

M18. The next set of questions considers your knowledge regarding malaria and malaria chemoprophylaxis medications.

For each question, please provide the "single" best answer. If you don't know the answer to the question, it is alright to say that you don't know. You may also refuse to answer the question. Do not hesitate to ask for help if you do not understand.

M18A. Have you ever heard of malaria? Not Gradable, General Knowledge
O. No
O Don't Know
O 1. Yes
O Refuse to Answer

M18B. Can a person get malaria from a mosquito bite?
O. No
Don't Know
O 1. Yes
O Refuse to Answer

M 18C. Can a person get malaria from blood transfusions?
O 0 . No
O Don't Know
O 1. Yes
O Refuse to Answer

M18D. Can malaria be transmitted from person to person by close contact or cough?
O 0. No
Don't Know
1. Yes
Refuse to Answer

M18E. Can malaria cause death?
O. No
Don't Know
1. Yes
Pefuse to Answer

M18F. Have you ever been prescribed malaria chemoprophylaxis medications? Not Gradable, General Knowledge
O 0. No
O Don't Know
O 1. Yes
O Refuse to Answer 
Appendix 4.2 - SPSS Cross Tab Output 


\begin{tabular}{|c|c|c|c|c|c|c|c|c|}
\hline & & & \multicolumn{5}{|c|}{ Intend to take the meds next time. } & \multirow[b]{2}{*}{ Tatal } \\
\hline & & & No & Yes & Don't Know & $\begin{array}{c}\text { Not } \\
\text { Applicable }\end{array}$ & $\begin{array}{l}\text { Refuse to } \\
\text { Answer }\end{array}$ & \\
\hline \multirow{35}{*}{$\begin{array}{l}\text { Plan malaria meds into } \\
\text { missions? }\end{array}$} & \multirow[t]{5}{*}{ None of the time, Never } & Count & 20 & 13 & 15 & 1 & 0 & 49 \\
\hline & & Expected Count & 8.6 & 29.9 & 7.6 & 2.6 & .3 & 49.0 \\
\hline & & $\begin{array}{l}\text { \% within W230. Plan } \\
\text { malaria meds into } \\
\text { missions? }\end{array}$ & $40.8 \%$ & $26.5 \%$ & $30.6 \%$ & $2.0 \%$ & $.0 \%$ & $100.0 \%$ \\
\hline & & $\begin{array}{l}\% \text { within m23H. Plan to } \\
\text { take them as directed? }\end{array}$ & $76.9 \%$ & $14.4 \%$ & $65.2 \%$ & $12.5 \%$ & $.0 \%$ & $33.1 \%$ \\
\hline & & $\%$ of Total & $13.5 \%$ & $8.8 \%$ & $10.1 \%$ & $.7 \%$ & $.0 \%$ & $33.1 \%$ \\
\hline & \multirow{5}{*}{$\begin{array}{l}\text { Some of the time, about } \\
25 \% \text { of the time }\end{array}$} & Count & 3 & 13 & 4 & 1 & 0 & 21 \\
\hline & & Expected Count & 3.7 & 12.8 & 3.3 & 1.1 & .1 & 21.0 \\
\hline & & $\begin{array}{l}\text { \% within m230. Plan } \\
\text { malaria meds into } \\
\text { missions? }\end{array}$ & $14.3 \%$ & $61.9 \%$ & $19.0 \%$ & $4.8 \%$ & $.0 \%$ & $100.0 \%$ \\
\hline & & $\begin{array}{l}8 \text { within m23H. Plan to } \\
\text { take them as directed? }\end{array}$ & $11.5 \%$ & $14.4 \%$ & $17.4 \%$ & $12.5 \%$ & $.0 \%$ & $14.2 \%$ \\
\hline & & $\%$ of Total & $2.0 \%$ & $8.8 \%$ & $2.7 \%$ & $.7 \%$ & $.0 \%$ & $14.2 \%$ \\
\hline & \multirow{5}{*}{$\begin{array}{l}\text { Most of the time, about } \\
50 \% \text { to } 75 \% \text { of the time }\end{array}$} & Count & 2 & 33 & 3 & 1 & 0 & 39 \\
\hline & & Expected Count & 6.9 & 23.7 & 6.1 & 2.1 & .3 & 30.0 \\
\hline & & $\begin{array}{l}\text { o within m230. Plan } \\
\text { malaria meds into } \\
\text { missions? }\end{array}$ & $5.1 \%$ & $84.6 \%$ & $7.7 \%$ & $2.6 \%$ & $.0 \%$ & $100.0 \%$ \\
\hline & & $\begin{array}{l}\text { \% within m23H. Plan to } \\
\text { take them as directed? }\end{array}$ & $7.7 \%$ & $36.7 \%$ & $13.0 \%$ & $12.5 \%$ & $.0 \%$ & $26.4 \%$ \\
\hline & & $\%$ of Total & $1.4 \%$ & $22.3 \%$ & $2.0 \%$ & $.7 \%$ & $.0 \%$ & $26.4 \%$ \\
\hline & \multirow{5}{*}{$\begin{array}{l}\text { All the time, } 100 \% \text { of the } \\
\text { time, Without exception }\end{array}$} & Count & 1 & 23 & 0 & 0 & 0 & 24 \\
\hline & & Expected Count & 4.2 & 14.6 & 3.7 & 1.3 & .2 & 24.0 \\
\hline & & $\begin{array}{l}\text { \% within m230. Flan } \\
\text { malaria meds into } \\
\text { missions? }\end{array}$ & $4.2 \%$ & $95.8 \%$ & $.0 \%$ & $.0 \%$ & $.0 \%$ & $100.0 \%$ \\
\hline & & $\begin{array}{l}\text { \% within M23H. Plan to } \\
\text { take them as directed? }\end{array}$ & $3.8 \%$ & $25.6 \%$ & $.0 \%$ & $.0 \%$ & $.0 \%$ & $16.2 \%$ \\
\hline & & of of Total & $.7 \%$ & $15.5 \%$ & $.0 \%$ & $.0 \%$ & $.0 \%$ & $16.2 \%$ \\
\hline & \multirow[t]{5}{*}{ Dont Know } & Count & 0 & 3 & 0 & 0 & 1 & 4 \\
\hline & & Expected Count & .7 & 2.4 & .6 & .2 & .0 & 4.0 \\
\hline & & $\begin{array}{l}\text { \% within M230. Plan } \\
\text { malaria meds into } \\
\text { missions? }\end{array}$ & $.0 \%$ & $75.0 \%$ & $.0 \%$ & $.0 \%$ & $25.0 \%$ & $100.0 \%$ \\
\hline & & $\begin{array}{l}\text { \% within m23H. Plan to } \\
\text { take them as directed? }\end{array}$ & $.0 \%$ & $3.3 \%$ & $.0 \%$ & $.0 \%$ & $100.0 \%$ & $2.7 \%$ \\
\hline & & $\%$ of Total & $.0 \%$ & $2.0 \%$ & .08 & $.0 \%$ & $.7 \%$ & $2.7 \%$ \\
\hline & \multirow[t]{5}{*}{ Not Applicable } & Count & 0 & 3 & 1 & 5 & 0 & 9 \\
\hline & & Expected Count & 1.6 & 5.5 & 1.4 & .5 & .1 & 9.0 \\
\hline & & $\begin{array}{l}\text { \%o within min } 230 \text {. Plan } \\
\text { malaria meds into } \\
\text { missions? }\end{array}$ & $.0 \%$ & $33.3 \%$ & $11.1 \%$ & $55.6 \%$ & $.0 \%$ & $100.0 \%$ \\
\hline & & $\begin{array}{l}\text { \% within M23H. Plan to } \\
\text { take them as directed? }\end{array}$ & $.0 \%$ & $3.3 \%$ & $4.3 \%$ & $62.5 \%$ & $.0 \%$ & $6.1 \%$ \\
\hline & & $\phi_{6}$ of Total & $.0 \%$ & $2.0 \%$ & $.7 \%$ & $3.4 \%$ & $.0 \%$ & $6.1 \%$ \\
\hline & \multirow[t]{5}{*}{ Refuse to Answer } & Count & 0 & 2 & 0 & 0 & 0 & 2 \\
\hline & & Expected Count & .4 & 1.2 & .3 & .1 & .0 & 2.0 \\
\hline & & $\begin{array}{l}\text { \% within m230. Plan } \\
\text { malaria meds into } \\
\text { missions? }\end{array}$ & $.0 \%$ & $100.0 \%$ & $.0 \%$ & $.0 \%$ & $.0 \%$ & $100.0 \%$ \\
\hline & & $\begin{array}{l}8 \text { within } \text { W23H. Plan to } \\
\text { take them as directed? }\end{array}$ & $.0 \%$ & $2.2 \%$ & $.0 \%$ & $.0 \%$ & $.0 \%$ & $1.4 \%$ \\
\hline & & $\%$ of Total & $.0 \%$ & $1.4 \%$ & $.0 \%$ & $.0 \%$ & $.0 \%$ & $1.4 \%$ \\
\hline \multirow[t]{5}{*}{ Total } & & Count & 26 & 90 & 23 & 8 & 1 & 148 \\
\hline & & Expected Count & 26.0 & 90.0 & 23.0 & 8.0 & 1.0 & 148.0 \\
\hline & & $\begin{array}{l}\text { \%o within m230. Plan } \\
\text { malaria meds into } \\
\text { missions? }\end{array}$ & $17.6 \%$ & $60.8 \%$ & $15.5 \%$ & $5.4 \%$ & $.7 \%$ & $100.0 \%$ \\
\hline & & $\begin{array}{l}\text { of within M23H. Plan to } \\
\text { take them as directed? }\end{array}$ & $100.0 \%$ & $100.0 \%$ & $100.0 \%$ & $100.0 \%$ & $100.0 \%$ & $100.0 \%$ \\
\hline & & $\%$ of Total & $17.6 \%$ & $60.8 \%$ & $15.5 \%$ & $5.4 \%$ & $.7 \%$ & $100.0 \%$ \\
\hline
\end{tabular}


VITA

MICHAEL J. PAGEL

October 1, 1963

1988 -Present

$1989-2008$

1991-1992

1992-1997

1995

1996

1997-1999

1999

2000-2001

2002
Born, Kingsville, Texas

US Army, Numerous Duty Stations

Bronze Star Medal

Defense Meritorious Service Medal Joint Service Achievement Medal

Army Commendation Medal

NATO Defense Service Medal

Special Forces Qualification Course JFK Special Warfare Training Center Fort Bragg, N.C.

B.S., Psychology

Regents College

Albany, New York

Ranger Course

US Army Ranger School

Fort Benning, GA

Distinguished Honor Graduate

Special Forces Underwater Operations

Dive Supervisor Course

Key West, FL

B.S., Physician Assistant Studies

University of Nebraska Medical Center

Omaha, Nebraska

Honor Graduate

US Army Officer Basic Course

Fort Sam Houston, TX

M.S., Physician Assistant Studies

University of Nebraska Medical Center

Omaha, Nebraska

Joint Medical Planners Course

Joint Staff and Naval Medicine Training Command

Bethesda, Maryland 
$2009-2012$

Doctoral Candidate

Florida International University

Miami, Florida

\section{PUBLICATIONS AND PRESENTATIONS}

Author, Initial Medical Treatment Policy - Detention Facility; Guantanamo Bay, Cuba, 2002

Initial Development of the US Southern Command Medical Evacuation Regulation, 2002

Author of over 30 Medical Capability Assessments throughout the Caribbean, Central America, and South America, 2001-2007

Strategic Medical Planner for Numerous Multinational Military and Joint Interagency Operations, 2002-2007

Author, Numerous Medical Readiness and Health Threat Publications, 2002-2007

Primary Orchestrator of two National Level Medical Training and Indoctrination events for the Country of Colombia, 2003 and 2004

Author, Joint Interagency Task Force - South, Medical Readiness Policy, 2004

Initial Planning and Implementation of the Special Operations Command - South Medical Clinic and Medical Support Operations Section, 2005 